SLAC-R-95-458

UC-414

\title{
STUDIES OF THE STRONG AND ELECTROWEAK INTERACTIONS AT THE $Z^{0}$ POLE*
}

\author{
Michael Douglas Hildreth \\ Stanford Linear Accelerator Center \\ Stanford Univergity, Stanford, CA 94309
}

March 1995

Prepared for the Department of Energy under contract number DE-AC03-76SF00515

Printed in the United States of Anerics. Available from the National Technical luformation Service, U. S. Department of Comnerce, 6285 Port Royal Roed, Springfield, Virginia 22161

Ph.D. Thesh 


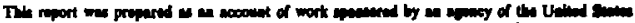

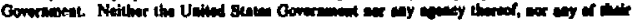

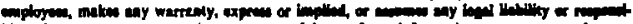

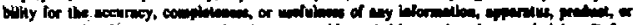

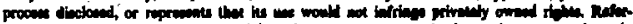

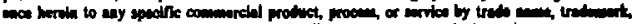

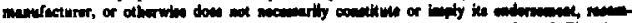

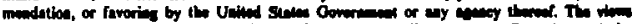

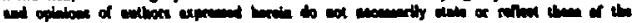

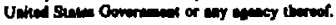

\section{Abstract}

This thesis presenta studies of the strong and electroneak forces, two of the fundsmeatal intersctions that govern the behnviour of matter at high energies. Wo bave usad the hadronic deceys of $Z^{0}$ bonons produced with the unigue experimental epperatus of the $e^{+} e^{-}$Linear Collider at the Stanford Linear Accelerator Center (SLAC) and the SLAC Large Detector (SLD) for these measurements. Employing the procision tracking copabilitied of the SLD, we isolated aumples of $Z^{0}$ evento containing primarily the decays of the $Z^{0}$ to a chosen quark type. With an inclusive selection technique, we have teated the flavor independence of the strong coupling, $a_{n}$, by measuring the rates of multi-jet production in isolated aunples of light (uds), $c$, and $b$ quark events. We find: $\alpha_{a}^{t} / \alpha_{*}^{t l}=0.987 \pm 0.027$ (stat) \pm 0.022 (syst) \pm 0.022 (theory),

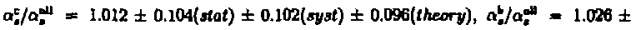
0.041 (stat) \pm 0.041 (syst) \pm 0.030 (theory), which implles that the strong interection is independent of quark favor within our present experimental sensitivity. We have also mossured the extent of parity-violation in the $Z^{0} c^{z}$ coupling, given by the parameter $A_{a}^{0}$, using a sample of fully and partially reconstructed $D^{\cdot}$ and $D^{+}$meson decays and the longitudinal polurization of the SLC electron beam. This anple of charm quark everts was derived with selection techniques basted on their kinernatic properties and decay topologies. Wa find $A_{c}^{0}=0.73 \pm 0.22$ (stat) \pm 0.10 (syst). This value is congiatent with that expected in the electroweak standard model of particle interactions. 
This thesis is dedicated to:

$$
\text { my grandfather }
$$

\section{Stanley 5 . Kotarshi}

(d. 1988)

for his constant support and encourugement May this make him prout...

and to

my son

Andrew. D. Hildreth

(b. 1994)

for recistablishing my wonder

at the mirscle of the universe

I wish they could have knoun cach other 


\section{Acknowledgements}

Ah, a chance to rellect on the halcyon days of graduate schoul... Believe it or not, studying physics here at Stanford has been more fun that just about anything else I've done. I guess that's why I've been called the "tefon graduste atudent" - none of the prototypically "bad" experiences of grad school life seemed to bother me. I even (sort-of) enjoyed the opportunity to study for the qual. Will, cnough about pain and torture...

I'd like to thank ail of those who have made my stay here a pleasant way to learn physics. First, a gracious thanks goes my advisor Davu Burke, who hes spent ix years leading me where, as it turned out, I really wanted to go anyway. Somehow, he always knows exactly which question to ask; he has taught me a great deal about how to visualizo a prohlem and attact its moit important parts. If I could take away with me half of his insight, I would be well olt. Next, my other physics mentors should be mentioned, since they have taken my broad interests in particle physics and stretched them even further. To Phil Burrows and Dave Muller, stalwart defenders of:the QCD realm, I offer my thanks and gratitude for their input to and interest in my work. Without their experience and knowledge, my studies of the strong interaction would have lackad direction and clarity. Both heve been good friends as well, and have been instrumental in introducing me to tbe finer things of life in California. To Steve Wagner, my partner in crime ("co-founder" sounds too stuffy) in the charm working group, I offer thanks for allowing me to share his immense knowledge and intultion about our charming mesons. I can eay that he has taugit me all of the charm physics I know. It's also been fun having anuther survivor of Mark II with which around with which to share the joys and tragedies of living with SLD software. I ghould also 
mantion here two of the current anchors of my old Group E. I would like to thenk John

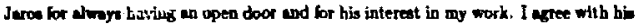
prowine that "If it's not simple; it ain't physica." He has taught me thoe and again that if I don't underatend womething intuitively, I probably don't uoder at and tt at all. Alo, I would libe to mention with gratitude the idea and conoments I'vo received over the years from Su Dong. His ineiglit into both of my analyse has certeinly unproved oar uoderotandire of tbe rubjects at hand. He ba been a conotant nource

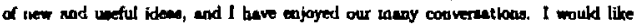
thank Micheel foskin for his tiretew enthurinan, bl seeningly infinite knowledge ebout mon phytics than I coukd hope to abeorb in three lifetimes, und for him cureful reading of this thesis, Thanke for keeping me boreat, Mkehel.

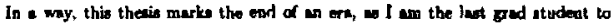
have been part of Murk II. I remenber my Mak II deye with foodpew, no doubt engendered by the way the group banded together in the face of constant idvernity. The old Mark Il grad students of those days, Dale Koetke and Bob Jecobeen, among others, have remained good triends; now I'll actually be able to do wome phyatce with you guys. Being part of the Mark II "experience" wa an unumul fuxury, as I have been abfe to spend time as part of two rompletely different colleboratione during my gradunte career, and have enjoyed both of them. I hope that I can atill harases my former mentor K. K. Gan from time to time.

Of course, this section wonid not be complete without thanking thoe folks who toil eadenly in pursuit of SLD perfection. Finat, I would like to thank tny SLD "cles. mate" Tom Junk, Homer Neal, and Tos Pavel, for enduring my constant quertions about vurious software urcana. They joined SLD before I did, and were extrennely patient with me a I got up to upeed. I wish then all godepeod in finlahing therr theses, end beet of luck in they venture out tmo the vet wee of postdocdom. I have vay much enjoyed the comaraderie of such a eronll collaboration (which hopefully will grow!), and I would like to repectfully thenk the highex-up types who run the ahoe (eapecially Merty Breidenbech) for allowing w to work in the give-and-take at mosphere that our aituntion dernundo.

I mould libe to expres my appreclation for all of the mpport l've recoived from 


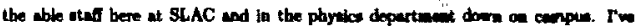

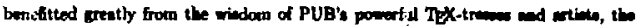

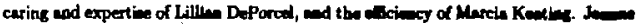

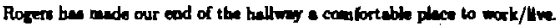

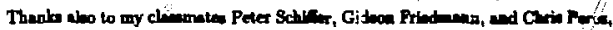

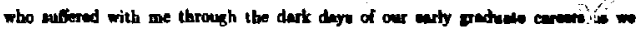

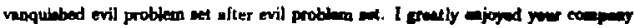

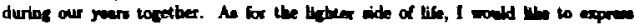

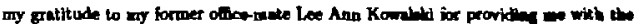
impetus to aing here at Stanlord. The folks of the Newmen Choir and the Symphomic Choru have brought mech joy to my life bere. I can't reelly inengipe whet 1 would

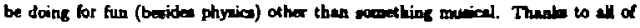
you for bringing that blewing into my tik. "f have had singing."

Actually, thin therin should beve four dedication, but I thed the symentry of

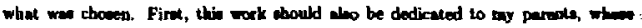

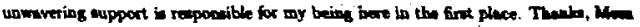
and Ded, for allowing me w folton what I thought we intereating and exciting rather then a proper caver. Holly's parents have aloo been extretoely mpportive of my endeavore, and 1 thank them tio.

The second and moot important dedication to to my wife Holly. Her companionship, love, and friendobip mean mose to me than anything. Without hor, this entics journey would have been rther shallow. Without her loving wpport and comfort, I enight not have made it. 


\section{Contents}

1 Overview 1

1.1 The Standard Model $\ldots \ldots \ldots \ldots \ldots$

2 The Electroweal Interaction: An Introduction

2.1 The Electroweak Standard Model . . . . . . . . . . . . . . . 6

2.1.1 Spuntaneous Symmetry Breaking; Tho Higs Mecharism . . . 9

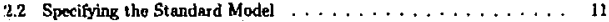

$2.3 Z^{0}$ Production and Decky . . . . . . . . . . . . 23

2.4 Renormalization of the Electroweak Coupllng . . . . . . . . . 16

2.4.) Solt Fudiative Corrections ............... 16

2.4.2 Hard Hediative Carections ............... 18

2.5 Electroweak Asymmetrics: Testing the Standard Model . . . . . . 24

2.5.1 The Lelt-Right Asymmetry .............. 25

2.5.c The $\tau$ Polatization Asymmetry ............. 26

2.5.3 The Forward-Backward Asymmetry . . . . . . . . . . 27

2.6 Radiative Corrections to $\bar{A}_{F B} \ldots \ldots \ldots \ldots \ldots$

2.6.1 Electroweak Corrections ................. 30

2.6.2 QCD Corrections ................... 32

2.7 Possible Modifications to $A$ from New Physics . . . . . . . . . 35

2.7.1 Two-Higzs Doublet Models and Supersymmetry . . . . . . 36

2.7 .2 Extended Gauge Sectors ................. 37

2.8 Measurements of the Heavy Quark Forward-Backward Asymmetries . 38

2.8 .1 High p. Lepton Tags . . . . . . . . . . . . . . . 39 
2.8.2 Exctunive Reconstruction of Charmed Mesopas . . . . . 41

2.8.3 Lifetime Tag Combined with Jet Charge . . . . . . . 44

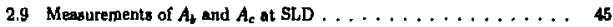

2.10 The Measurement of $A_{\varepsilon}$ Usirg Excluaively Reconstructed $D$ Mescons . 47

3 The Strong Interaction: An Introduction

3.1 Quartum Chromodytumics $\ldots \ldots \ldots \ldots \ldots \ldots \ldots$

3.1 .1 The QCD Lógrangian . . . . . . . . . . . . . . 51

3.1 .2 Simple QCD Proceses $\ldots \ldots \ldots \ldots \ldots \ldots \ldots$

3.1.3 The Renormalization of the Strong Coupling . . . . . . . 57

3.1.4 The Alturelli-Parisi Equations: The Leading Logarithm Ap-

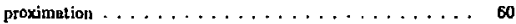

3.1.5 Higher Order Corrections: Tue Renormalization Group Equation 61

3.1.6 Asymptotic Freedom and Quark Confinement . . . . . 63

3.2 Production of Hadrons in $e^{t} e^{-}$Collisions $\ldots \ldots \ldots \ldots \ldots \ldots$

3.2 .1 The Fegmentation Process $\ldots \ldots \ldots \ldots \ldots \ldots \ldots 6$

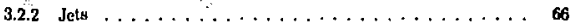

3.2.3 Simulations of Fragmentation $\ldots \ldots \ldots \ldots \ldots \ldots 67$

3.3 Meusurements of $\alpha_{1} \ldots \ldots \ldots \ldots \ldots \ldots \ldots \ldots \ldots$

3.3.1 Jet Rates $\ldots \ldots \ldots \ldots \ldots \ldots \ldots \ldots \ldots \ldots \ldots$

3.3.2 Event Shape Variables and Particie Correlations . . . . . 00

3.4 Tests of $Q C D \ldots \ldots \ldots \ldots \ldots \ldots \ldots \ldots \ldots \ldots$

3.4.1 Flavor Independence of $\alpha_{1} \ldots \ldots \ldots \ldots \ldots \ldots$

4 Experimental Appuratus: the SLC and SLD of

4.1 The SLAC Linear Collider. . . . . . . . . . . . . . 9l

4.1.1 The Polarized Eloctron Source . . . . . . . . . . . . 93

4.1.2 Beam Transport . . . . . . . . . . . . . . 98

4.1.3 $S_{p i n}$ Trunsport $\ldots \ldots \ldots \ldots \ldots \ldots \ldots \ldots \ldots \ldots$

4.1.4 Beam Energy Measurement . . . . . . . . . . 96

4.1.5 Beam Polarization Measurement . . . . . . . . . . 97

4.1.6 SLC Performance History $\ldots \ldots \ldots \ldots \ldots \ldots \ldots$ 
4.2 The SLC Large Detector . . . . . . . . . . . . . . . 102

4.2.1 The VXD and Banpipe . . . . . . . . . . . . . 103

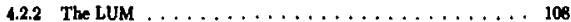

4.2.3 The CDC $\ldots \ldots \ldots \ldots \ldots \ldots \ldots \ldots \ldots \ldots$

1.2.4 The CRuD $\ldots \ldots \ldots \ldots \ldots \ldots \ldots \ldots \ldots \ldots \ldots \ldots \ldots 112$

4.2.5 The LAC $\ldots \ldots \ldots \ldots \ldots \ldots \ldots \ldots \ldots \ldots \ldots$

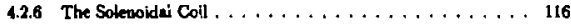

4.2.7 The w10 $\ldots \ldots \ldots \ldots \ldots \ldots \ldots \ldots \ldots \ldots \ldots$

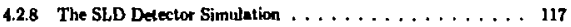

5 Trecking Syatem Performance 119

5.1 Treck Reconstruction . . . . . . . . . . . . . . 110

5.2 Determination of the Prlmary Vertex (IP) Position $\ldots .127$

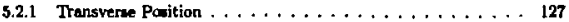

5.2 .2 Longitudinal Position . . . . . . . . . . . 133

5.3 The Impect Parameter Distribution . . . . . . . . . . . 133

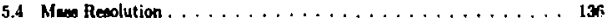

5.5 Vertex Finding $\ldots \ldots \ldots \ldots \ldots \ldots \ldots \ldots \ldots \ldots$

6 Hadronic Event Solection 141

6.1 The SLD Trigger $\ldots \ldots \ldots \ldots \ldots \ldots \ldots \ldots \ldots \ldots \ldots \ldots \ldots$

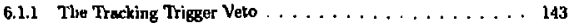

6.2 The Hadronic Event Fitter . . . . . . . . . . . . 144

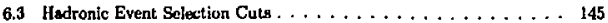

6.3.1 Event Selection for the a, Analysis . . . . . . . . . . 146

6.3.2 Event Selection for the $A_{c}$ Analyzis $\ldots \ldots \ldots \ldots \ldots \ldots$

1 A Test of the Flavor Independence of the Strong Interaction $\quad 151$

7 Flavor Tagsing 152

7.1 Selection of Guality Tracks . . . . . . . . . . . 152 
7.2 The Normalised Impact Parameter . . . . . . . . . . . 15

7.3 Definition of the Flawor Trap $\ldots \ldots \ldots \ldots \ldots \ldots$

8 : Jot Finding 14

8.1 Jet Algorithm Definitions $\ldots \ldots \ldots \ldots \ldots \ldots \ldots \ldots$

8.1 .1 The E Scheme . . . . . . . . . . . . . . 160

8.1.2 The Eo Scheme $\ldots \ldots \ldots \ldots \ldots \ldots \ldots$

8.1.3 The P Scheme . . . . . . . . . . . . . . 16s

8.1.4 The PO Scheme $\ldots \ldots \ldots \ldots \ldots \ldots \ldots$

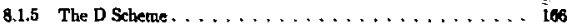

8.1.6 The $\mathrm{G}$ Scheme $\ldots \ldots \ldots \ldots \ldots \ldots \ldots$

8.2 Definition of the $n-J e t$ Rate and $D_{2} \ldots \ldots \ldots \ldots \ldots$

8.3 QCD calculation for Jet Rates and $D_{2} \ldots \ldots \ldots \ldots \ldots$

8.4 The Need for Corrections. . . . . . . . . . . . . . 100

9 Annlyaia Method 174

9.1 Coneiderations $\ldots \ldots \ldots \ldots \ldots \ldots \ldots \ldots \ldots \ldots \ldots$

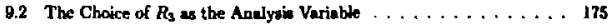

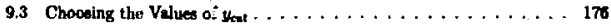

9.4 Fitting the Data for $R_{3} \ldots \ldots \ldots \ldots \ldots \ldots$

9.5 Tag Bias $\ldots \ldots \ldots \ldots \ldots \ldots \ldots \ldots \ldots \ldots \ldots \ldots$

9.6 Results for $R_{3}^{j} \ldots \ldots \ldots \ldots \ldots \ldots \ldots$

10 From Jet Rntes to $a$, 101

10.1 Phase-Space Supprcssion of Gluon Rediation . . . . . . . . 191

10.2 Ohtaining the Velues for $\alpha_{\mathfrak{y}} / a_{a}^{\text {dt }} \ldots \ldots \ldots \ldots \ldots \ldots \ldots$

11 Syntematic Errors and Consintency Cbecks 16

11.1 Experimental Errors $\ldots \ldots \ldots \ldots \ldots \ldots \ldots \ldots \ldots \ldots$

11.1.1 Event Re-weighting . . . . . . . . . . . . 190

11.1.2 Errors due to $b$-Hodron Mordeling $\ldots \ldots \ldots \ldots \ldots$

11.1.3 Errors due to c-Hedron Modeling . . . . . . . . . 203 
11.1.4 Other Exporinental Errors. . . . . . . . . . . . 205

11.2 Theoretical Uncertainties . . . . . . . . . . . . . . . 209

11.3 Checks on the Validity of the Resuits . . . . . . . . . 212

11.3.1 Variacion of the Choeen yow . . . . . . . . . 213

11.3.2 Varintion of the Tag Definition . . . . . . . . 213

11.3.3 Seruitivity to Anomalous Flavor-Dependent Strong Couplings 216

12 The Flavor Independence of $\alpha$; Conclumions 219

12.1 Final Results and Dincussion . . . . . . . . . . 219

12.2 Possibie Improvementa of the Method . . . . . . . . . . 223

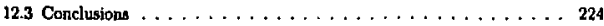

II A Measurement of the Parity Violation in the $Z^{0} c c$ Coupling 226

13 Anelyais Method 220

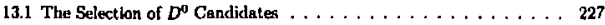

13.1.1 The Kinemetle Txchnique . . . . . . . . . 22B

13.1.2 The Decay Length Technique . . . . . . . . . . 232

13.2 The Selection of $D^{+}$Candidates . . . . . . . . . . 239

13.3 Determination of the Background and Signal Fractions $\ldots \ldots \ldots, 243$

13.4 The Rnw Anymmetry . . . . . . . . . . . . 247

14 The Arymmetry Likellhood Function 254

14.1 Formulation of the Likelitood Function $\ldots \ldots \ldots \ldots \ldots \ldots 254$

14.2 Inputs to the Likelihood Analysis . . . . . . . . . . 256

14.2.1 Determination of Asymmetrie . . . . . . . . . . 256

14.2.2 Formulation of the Functions $P(x) \ldots \ldots \ldots \ldots .258$

14.2.3 Other Inputs . . . . . . . . . . . . . 261

14.3 Results of the Fit $\ldots \ldots \ldots \ldots \ldots \ldots \ldots \ldots \ldots$

14.4 Checks of Fit Value $\ldots \ldots \ldots \ldots \ldots \ldots \ldots$. $\ldots \ldots 3$ 
16 Rudiative Corrections: Obtaining $A_{c}^{0}$

15.1 Electroweak Correction $\ldots \ldots \ldots \ldots \ldots$

15.2 QCD Corrections $\ldots \ldots \ldots \ldots \ldots \ldots \ldots$

16 Syatematic Errort 20

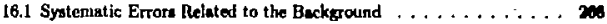

16.1.1 Determination of the Background Fraction . . . . . . 240

16.1.2 The Background Asymmetry . . . . . . . . . . . 273

16.1.3 Background Acceptance $\ldots \ldots \ldots \ldots \ldots \ldots \ldots \ldots .274$

16.1.4 Background $x$ Distribution . . . . . . . . . . 275

16.2 Systematic Errors Related to the Signal . . . . . . . . . 275

16.2.1 Determiuntion of the Signal Composition . . . . . . 275

16.2.2 Variation of the Value for $A_{b \rightarrow-0} \ldots \ldots \ldots \ldots \ldots$

16.2.3 Fragmentation Function Variation . . . . . . . . . 278

16.3 Uncertainties fron Outside Parameters . . . . . . . . 280

16.3.1 Uncertainty on $A_{\varepsilon} \ldots \ldots \ldots \ldots \ldots \ldots$

16.3.2 Polsrization Uncertainty . . . . . . . . . . 281

16.3.3 Uncertsinty in the Value of $a_{0}\left(A R_{2}^{2}\right) \ldots \ldots \ldots \ldots \ldots$

16.3.4 Uncertalnty in the $O\left(\alpha_{3}^{2}\right)$ QCD Correction . . . . . . 281

17 Conclunions 203

17.1 Final Result $\ldots \ldots \ldots \ldots \ldots \ldots \ldots \ldots \ldots \ldots \ldots \ldots$

17.2 Comparison with Existing Measurements . . . . . . . 283

17,3 Determination of $\operatorname{tin}^{2} \boldsymbol{\theta}_{w}^{f f}$ from $A_{c} \ldots \ldots \ldots \ldots \ldots \ldots$

17.4 Possible Improvernents $\ldots \ldots \ldots \ldots \ldots \ldots \ldots \ldots \ldots$

A Efficiency Matrice for $R_{3}$ Unfolding 280

B Coefficients for the Jet Rntes Calculation

C The Mathematical Formulntion for Unfolding $h_{3}^{j} \quad 297$ 
D Accolerator Becterounds at the SLC $\mathbf{5 0 0}$

D.1 The SLC Optics and Collimation Syatem . . . . . . . . . . . . 301

D.2 Typee of Beckground Seen in the SLD Detector . . . . . . . . 308

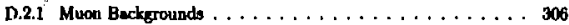

0.2.2 Synchrotron Pediation Backgroundo . . . . . . . . 310

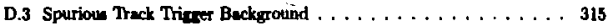

D.4 SR Beckground Mewurements . . . . . . . . . . . 318

D.5 Trecking Trigger Backgounds Studies $\ldots \ldots \ldots \ldots \ldots \ldots \ldots \ldots$

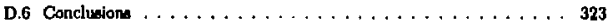

E The SLD Collnboration 329 


\section{List of Tables}

1.1 The funderrental fermions of the Stundard Model. . . . . . . . 3

2.1 The $L$ isodoublete and $R$ iosinglets of $S U(2)_{l} \times V(1)_{Y} \ldots \ldots, 7$

2.2 The parameters which specify the electrowenk Standard Model it tre level $\ldots \ldots \ldots \ldots \ldots \ldots \ldots \ldots \ldots \ldots$

2.3 The vector and axial vector couplings for the fetmions to the $Z Z^{0} \ldots 14$

2.4 A conparieun of the different observable mymutsies . . . . . 20

2.5 The largest branching frections in $D^{a}$ decay $\ldots \ldots \ldots \ldots \ldots$

2.6 The iargest branching fractions in $D^{+}$decay. . . . . . . . 49

5.1 Tracking Rewolution . . . . . . . . . . . 123

6.1 Hadronic Event Selection Efficlenciea $\ldots \ldots \ldots \ldots \ldots$

6.2 Hedronic Multijet Event Selection Efficiencles . . . . . . 14

9.1 Tog Biases for Eech Algorithr $\ldots \ldots \ldots \ldots \ldots \ldots \ldots \ldots$

9.2 Input Data for the $R_{3}$ Fit $\ldots \ldots \ldots \ldots \ldots \ldots$

0.3 Results for $\mathbb{R}_{3}^{j} / R_{\mathbf{3}} \ldots \ldots \ldots \ldots \ldots \ldots \ldots \ldots \ldots$

10.1 $R_{J}^{j} / R^{\ell}$ Values Corrected for Phase Space. . . . . . . . . 195

10.2 Values of $a_{0}^{a t}$ and $f$ Used . . . . . . . . . . . . . 197

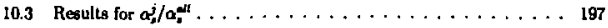

11.1 Systernatic Errors Due to $b$ Hedron Modeling . . . . . . . . 203

11.2 Systematic Errors Due to $\mathrm{c}$ Hadron Modeling . . . . . . . . 205

11.3 Systematic Errors Due to Variation of Tage and Cuts . . . . 208

11.4 Systematic Errors Due to Detector Madeling . . . . . . . 200

11.5 Theoretical Uncerteinties $\ldots \ldots \ldots \ldots \ldots \ldots \ldots \ldots \ldots \ldots$

11.6 Summary Table of Systematic Errors . . . . . . . . 212 


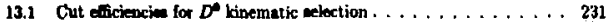

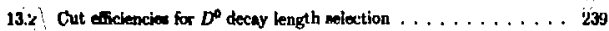

13.3 Cut estection eficiencies for the $D^{+}$sulyais $\ldots \ldots \ldots \ldots \ldots 243$

13.4 Tolal number of ajgal eventu and signal composition . . . . 251

13.5 Total number of sideband events and sideband composition . . . . 251

15.1 Frection of QQQQ or $9 Q Q Q$ events pasing cuts. . . . . . . 266

16.1 The number of double tagged $D^{0}$ decays in data and $M C \ldots \ldots 271$

16.2 The number of double-tagged $D^{\circ}$ decays in data and MC after correction273

16.3 Exicting messurements of $D^{+4}$ and $D^{+}$production in $Z^{0}$ decays. . . 276

16.4 The variation of $\omega_{b}$ for different veluca of $F_{b} \cdot P_{b \rightarrow D} / F_{c} \cdot P_{c \rightarrow D} . \quad \ldots 277$

16.5 Contributions to tl se estimated systematic error on $A_{c}^{0} \ldots \ldots \ldots 282$ 


\section{List of Figures}

2.1 The totel croes section for $e^{+} e^{-} \rightarrow \mu^{+} \mu^{-}$vi. center-of- onesenetgy , 15

2.2 The leading-order initital state $Q E D$ corrections to the $\mathbb{J}^{0}$ resonance

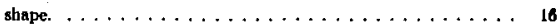

2.3 Variation of the $\boldsymbol{Z}^{0}$ resonance shapo from ISR (sec text). . . . . 17

2.4 A schematic viow of the different electromeak radiative corrections . 18

2.5 A schematic view of the photon self-energy corrections . . . . 19

2.6 A schematic view of the different vector boson self-energy corrections. 21

2.7 A schematic view of the different vector bowon self-energy corrections. 21

2.8 The deperdence of $\sin ^{2}$ off $w^{\prime}$ on $m_{1}$ and $m_{\text {Hispe }} \ldots \ldots \ldots \ldots . \ldots 23$

2.9 Electrowenk-corrected values of $A_{b}$ and $A_{c}$ sear the $Z^{\circ}$ pole . . . . 31

2.10 First-order QCD cortections to $A_{b}$ and $A_{e} \ldots \ldots \ldots \ldots \ldots$

2.11 Radiative Corrections to $A_{6}$ from virtual top quark icops. . . . 35

2.12 The $p$ and $p_{\perp}$ distributions for leptons in hadronic $Z^{0}$ decays. . . .

2.13 The left-right forward-beckward anymetry for high $p, p_{\perp}$ leptons. . 45

$2.14 b$ quark production asymmetries from Jet Charge $\ldots \ldots \ldots \ldots$

3.1 The Interactions contained in the QCD lagrangian . . . . . . 52

3.2 Feynmen diagrarns for $e^{+} e^{-} \rightarrow q \ldots \ldots \ldots \ldots \ldots \ldots$

3.3 Feynman dingrams for $e^{+} e^{-} \rightarrow q \$ \ldots \ldots \ldots \ldots \ldots \ldots$

3.4 Feynman diagresus for $e^{+} e^{-} \rightarrow q \phi q \bar{q}$ and $e^{+} e^{-} \rightarrow q \bar{q} g g . \ldots \ldots \ldots, 55$

3.5 The leading order corrections to $\alpha_{0} \ldots \ldots \ldots \ldots \ldots \ldots$

3.6 An overview of an $e^{+} e^{-} \rightarrow q \bar{q}$ event $\ldots \ldots \ldots \ldots \ldots \ldots$

3.7 Views of 2 - and 3 -jet events in SLD $\ldots \ldots \ldots \ldots \ldots$

3.8 Parton Shower evolution $\ldots \ldots \ldots \ldots \ldots \ldots \ldots \ldots$ 
3.9 Schematic represcntation of Independent Fragmentation . . . . . 72

3.10 Schematic representation of String Fragmentation . . . . . . . . 74

3.11 A qig ovent in the string fragmentation weheme. . . . . . . . 75

3.12 A dingran of Cluster Eragmentalion. $\ldots \ldots \ldots \ldots \ldots \ldots$

3.13 An Illustration of the Ward-Takaheahi Idontity . . . . . . . . . . 84

4.1 The Polarized SLC ..................... 92

4.2 Energy State Dingram for GaAs . . . . . . . . . . . B4

4.3 The WisRD ..................... 96

4.4 The Conton Polarimeter System . . . . . . . . . . . 98

4.5 The Mcusured Compton Asymmetry $\ldots \ldots \ldots$

4.6 S:C Luminosity Performance History $\ldots \ldots \ldots \ldots \ldots$

4.7 IP Benu Size History . . . . . . . . . . . . . . . 101

$4.81991-$ L993 tritegrated Luminosity . . . . . . . . . . . . . . 102

4.9 The SLD Detector: An tsometric View . . . . . . . . . . . 103

4.10 The SLD Detcetor: A Quadrant View . . . . . . . . . . . . . 104

4.11 The VXD ...................... 106

4.12 The LUM ................................ 107

4.13 The Superlayer Structure of the CDC . . . . . . . . . . . 109

4.14 The CDC Jet Gell . . . . . . . . . . . . . . . . 110

4.15 Local and Globel CDC Drift Distnuce Reanlution . . . . . . . . 112

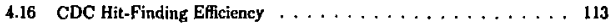

4.17 The CDC 2-hit Resolution ................... 114

4.18 The CRID ........................ 115

4.19 The LAC Tile Structure ................... 116

4.20 The WIC Structure . . . . . . . . . . . . 117

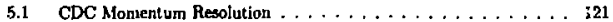

5.2 CDC Two Track Mis- Distences for the $z-y$ and $r-z$ Planes . . . . 122

5.3 GDC to VXD Linking Efficiency for Good Thacks vs. p. . . . . . . . 123

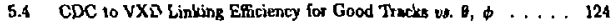

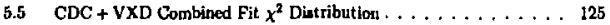

5.6 Derived luupact Parameter Resolutions . . . . . . . . . 126 
5.7 Fitting the Event IP Powition $\ldots \ldots \ldots \ldots \ldots \ldots \ldots$

5.8 Impact Parwneters of Mwon to (IP) $\ldots \ldots \ldots \ldots \ldots \ldots$

6.9 The $y_{r}$ dietribution for all events . . . . . . . . . 131

5.10 The $y_{r}$ diatribution for events with all tracks in the vertex. . . . 138 .

$5.11 x-y$ Normalized Impact Paraneters . . . . . . . . . . . 13

6.12 Ratio of Unumeared MC to data Normalized Impect Parameter Dir tributions $\ldots \ldots \ldots \ldots \ldots \ldots \ldots \ldots \ldots \ldots \ldots \ldots, 136$

$5.13 K_{0}^{0}$ and $\Lambda$ Mas Dhatrilustione $\ldots \ldots \ldots \ldots \ldots \ldots \ldots$

5.14 The Number of Two-Track Vertices Found in Hedronic Events . . . 138

5.15 The Decuy Length Resolution for $D^{+}$Decrya $\ldots \ldots \ldots \ldots \ldots$. $\ldots 39$

6.1 Thrust Axis Direction Determination: LAC v. CDC . . . . . 147

6.2 Distribution of Event Selection Cut Variables . . . . . . . 148

7.1 Diatributions of Qudity Track Selection Cut Variables in the MC . 154

7.2 Distributions of Track Selection Cut Veriabtes in the MC and data , 153

7.3 The Quality Track Multiplicity Dintrilutions . . . . . . . 15 s

7.4 Dizgram of a B Meion Decay Caucede . . . . . . . . 157

7.5 Inpect Parameter Signing . . . . . . . . . . Iso

7.6 Impact Parameter Distributions for uds, $c$, and $b$ Events . . . . . 160

7.7 The Number of Significant Tracks per Event . . . . . . . 163

8.1 Jet Rutes for All Algorithms . . . . . . . . . . 168

8.2 $D_{2}$ Distribution for the Pre veto Period Taged Sumples $\ldots \ldots .171$

$8.3 D_{2}$ Distribution for the Veto Perion Tagged Simples . . . . . . . 172

8.4 $D_{2}$ Distribution for the Non-veto Peried Tagged Sanples . . . . 173

9.1 The $D_{3}$ Distribution and $\mathcal{O}\left(a_{3}^{2}\right)$ Calculation for the $\mathrm{E}$ algorithm . 178

9.2 The $D_{2}$ Distribution and $\mathcal{O}\left(\alpha_{3}^{2}\right)$ Calculation for the EO algorithan . . 179

9.3 The $D_{2}$ Distribution and $O\left(\alpha_{3}^{2}\right)$ Calctiation for the $P$ algorithm .. 180

9.4 The $D_{2}$ Distribution and $O\left(\alpha_{s}^{x}\right)$ Cakulation for the P0 algorithm . . 181

9.5 The $D_{2}$ Distribution and $O\left(\alpha_{\text {g }}^{2}\right)$ Cakculation for the $D$ algorithrn . . 182

9.6 The $D_{2}$ Distribution and $O\left(a_{n}^{2}\right)$ Cakeulation for the $G$ algorithm . . 183

10.1 Phese Space Suppreasion Factors for eharm quarks . . . . . . 193

10.2 Phese Spece Suppression Fuators for b quarks . . . . . . . 19 
10.3 Conversion from $R_{3}$ ration to $\alpha$, ratios $\ldots \ldots \ldots \ldots$

11.1 Event Weights for the $b$ Fromentation Function . . . . . 202

11.2 The Results . rs. Input $R_{c} \ldots \ldots \ldots \ldots \ldots \ldots \ldots \ldots \ldots$

11.3 The Resuits vs. you Choice for E, E0, and $P \ldots \ldots \ldots \ldots 214$

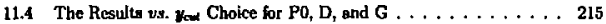

11.5 The Resulte vs. Track Significance Requirement . . . . . . . . . 216

11.6 The Resultu vs. Track Significance Requirement . . . . . . . . 217

11.7 Sensitivity to Input Anomolous Strong Coupling . . . . . . 218

12.1 Final results for $\alpha_{s}^{\$} / \alpha_{n}^{\text {all }}$ for each algorithm $\ldots \ldots \ldots \ldots \ldots 220$

12.2 Comparison of all results for $\alpha_{g}^{j} / \alpha_{f}^{n t} \ldots \ldots \ldots \ldots \ldots \ldots \ldots 22$

13.1 The invariant mass of $k x$ pairs after cuts. . . . . . . 228

13.2 Definition of the helicity angle $\theta^{*} \ldots \ldots \ldots \ldots \ldots \ldots$

13.3 Helicity angle distribution for $D^{\alpha} \rightarrow \mathfrak{k} \pi$ decays and background. . . 230

13.4 Slow pion momentum spectrum. . . . . . . . . . . 231

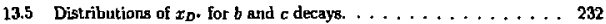

13.6 The $\Delta m$ distribution for the kimematic selection analysis. . . . . 233

13.7 The $x^{2}$ vertex probab"...y for falke vertices. . . . . . . . 234

13.8 Distributions of normalized decay length for true and fake vertices. . 235

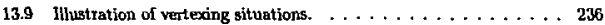

13.10 The impact parantiers of $D^{0}$ mesons to the IP $(D \rightarrow K \pi) \ldots \ldots 237$

13.11 The $\Delta m$ distributions for the decay lengtb analysis. . . . . . . 238

13.12 The $\cos \theta^{\circ}$ distribution for $D^{+}$decays. . . . . . . . . 240

13.13 The nngle between the $D^{+}$momentum and its flight direction. . . 241

13.14 The distribution of $K \pi \pi$ masses. . . . . . . . . . . . . 242

13.15 The $\Delta m$ and $D^{+}$mass distrihutions for date and MC. . . . . . 245

13.16 The $\Delta m$ distribution for data, MC signal and RCBG for $D^{0} \rightarrow K \pi .248$

13.17 The Am distribution for data, MC signal and RCBG for $D^{0} \rightarrow K \pi \pi^{0} .249$

13.18 The mee distribution for data, MC signal and RCBG for $D^{+} \rightarrow K \pi \pi .250$

13.19 The direction of the outgaing $D^{(*)+}$ (or the two different bears helicities.251

13.20 The forward beckward asymmetry, combining the two polarization states. . . . . . . . . . . . . . . . . 253 
14.1 Possible sources for $D$ menons in $B$ decays. . . . . . . . 257

14.2 The $x_{D}$ distributions for dato, beckground, and MC aignal . . . . 200

14.3 The MC distributions of $x_{c \rightarrow p}$ and their fitted perametrizations. . . 261

14.4 The MC distributions of $x_{b \rightarrow p}$ and their fitted perametrizations. . . 202

14.5 The minimization of the liketibood function. . . . . . . 263

14.6 Results of fitting for $A_{e}$ with a binred aymmetry. . . . . . 204

16.1 The MC background distribution for the $D^{+}$analyais. . . . . . 290

16.2 A third order polynomial beckground fit to the $D^{+}$signal and beckground. . . . . . . . . . . . . . . 272

16.'J Comparison of sigatal and sideband acceptances. . . . . . . . 274

16.4 The MC distributions for nominal, soft, and bard charm fraginentation.279

17.1 A comparison of the world's measurements of $A_{\tau^{*}}^{0} \ldots \ldots \ldots \ldots 28$

17.2 The dependence of $\sin ^{2} \theta_{W}^{\prime \prime}$ on $A_{r} \ldots \ldots \ldots \ldots \ldots \ldots \ldots$

D.1 An overview of the SLC collimation system . . . . . . . 301

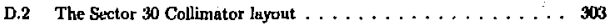

D.3 The 51-Line collimators . . . . . . . . . . . . . . 304

D.4 The Reverse-Bend collinators . . . . . . . . . . 304

D.5 Apertures in the Final Focus collimation system . . . . . . 305

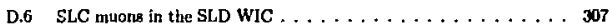

D.7 SLC muons in the SLD WTC . . . . . . . . . . 308

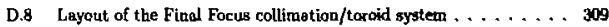

D.9 A diagram of the masking around the SLC IP . . . . . . . 311

D.10 Mensured and calculated CDC mespancies from the 1993 run . . 314

D.11 The $z$ priniary vertex posit ; , $z$ in lisw-nultiplicity events . . . . 316

D.12 The $x-y$ primary vertex positíntis in low-multiplicity events . . . 317

D.13 Measured and calculeted CDC occupancies from backgrounds studies 320

D.14 The CDC during low $B$ field running $\ldots \ldots \ldots \ldots \ldots \ldots$

D.15 Possible source of track-trigger backgrounds . . . . . . . 324 


\section{Chapter 1}

\section{Overview}

This thesis presents two studies of the physics encompasend in the Stundard Model of particle physics. The first study is a lest of the flavor independence of strong interactions, a fundanental assumption of the theory of strong interactions, Quantum Chromo-Dynamics (QCD). The accond analysis represents the first direct eneasurement of parity violation in the coupling of the $Z^{0}$ bason to clarm quasks. The data used to perform tlıcese investigations was accjuired at the Stanford Linear Accolerator Center (SLAC) using the SLAC Lerge Detector, (SLD) at the SLAC Linear Collider (SLC) during the calcudar year 1993. All of the data uas produced at a center-of-merss cnergy equal to the $Z^{0}$ boson mass.

The thesis begins with introductory chapters which present ant overvicw of the strong und clectrourak interactions that make up the Standard Model. Chapters follow which contah descriptions of the detector and accelerator apparatus and ity performance which are relevant to lotlt aublyses. At thio point, the thesig is divided into two parts, the first containing a deacription of the andysis roungrising the test of QCD in its entirety, the second coptuining the measurenent of the parity violation in the coupling of the $Z^{0}$ boesn to charin cilarke. Supporting appendices follow, adding complementary infornation to thei presented in the precediug chapters. In perticular. Appendix C. preacists an overview of the types of accelerator-induced backgrounds that can be encountered at a linear collider and various techuiques for their reduction.

The following sertion gives a brief layman's overview of the components of what 
hus become known as the Standarl Model of particle physics. Thoee portions relevaux to thls thes/s witl be iptroduced in much greater detal in the following chupters.

\subsection{The Standard Model}

In standard usage, the term "Gtandard Model" refers to the quantum field theories of the electroweak' and strong interactions that deacribe the interactions between the fundamental particles that nuake up the unlverse.

These fundamental particles, all of which are fermions (spin $\frac{1}{2}$ ), are divided into quarks and leptons. Quarks carry electromeak and colos charges, und thu interact via both the electroweak and atrong interactlons; thoy combine to form compoits particles called hadroma like the protun and neutron. The leptons consigt of particlea like the electron which carry clectroweak charges, and neul rinos whilch only carry the weak elharge.

The fundamental forces of unture are represented in these theories by vector (spin 1) booons which medipte the interactions betwen the fusudusuental ferrions. The electrowenk force containg four such bowons: the massless photon $(\gamma)$, which mediates the familliar electromagnetic force, the neutral $Z^{0}\left(m_{2} \sim 91 \mathrm{GnV} / c^{2}\right)$, and the charged $W^{+}$and $W^{-}\left(m_{W} \sim 80 \mathrm{GeV} / c^{2}\right)$, all of which are regponsilule for the weak force. The strong interactions are mediuted by massles partiches called gluons.

The one ingredient missing front the abowe description ls some means by which the masess of the fundamental fermions and bosons can be generated. This addition to the theoretical framework is commonly referred to tat the Higgy mechaniam. Its effect will be clincussed in detail in the next chapter.

As a sumniary, the fermions of the theory are listet in Tnble 1.1.

\footnotetext{
${ }^{1}$ The electrowet force contaln a deacription of the elecromagnetie and weak forces in a con-

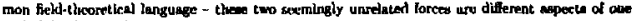
underlyins interaction.
} 
Table 1.1: The fundamental fernions of the Standaxd Model, with their electric charges and approximate masses in units of $\mathrm{GeV} / \mathrm{c}^{2}$.

\begin{tabular}{|cccc|}
\hline Charge & \multicolumn{3}{c}{$\begin{array}{c}\text { Qhunrike } \\
\text { Naune (Mass) }\end{array}$} \\
\hline$+\frac{7}{3} e$ & $u(\sim 0.1)$ & $c(\sim 1.5)$ & $t(\sim 175)$ \\
$-\frac{1}{3} e$ & $d(\sim 0.1)$ & $s(\sim 0.3)$ & $b(\sim 5)$ \\
\hline & & Leptons \\
Charge & & Name (Mass) & \\
\hline$-1 e$ & $e(\sim 0.05)$ & $\mu(\sim 0.1)$ & $\tau(\sim 1.7)$ \\
0 & $\nu_{e}\left(<\sim 10^{-0}\right)$ & $\nu_{\mu}(<\sim 0.003)$ & $\nu_{r}(<\sim 0.03)$ \\
\hline
\end{tabular}




\section{Chapter 2}

\section{The Electroweak Interaction:}

\section{An Introduction}

Beçiuerel's conjecture• that Roentgen's $\mathrm{X}$ rays[2] may have some relation to phosphoresenee in uranic salts has turned out to be true, but not in any way he could have imngined at the time. Becnungel'n 189 siscovery of what is nuw called radioactivity[3] while pursuing this question marks the starting point of the study of subatomic plysics as well as the first experitnental manifestation of the veale nuclear force. Since this result predates cven the churacterization of the electron as a fundumental particle[4] by a fow yearst, it is almust certain that the truc implications of this discovery were incomprehensible ot the time.

The discovery and subeequent claracte:ization of radinuctivity, however, Lave functioned us major catalysts for the cnormous growth in our underatanding of the substomic world that has occurred over the pust century. Consideration of $\beta$ decay s!ectra in conjunction with the unsolved problem of nuclear structure led Pauli to propose the oxistence of the electron nuutrino(6) in 1930; Fermi provided the first "correct" description of the low-energy weak interaction couched iu the language of quantum field theory anly three years later|7]. Out of al! of the developments that unfolded over the noxt two decades, the most striking is the discovery in 1957 that

\footnotetext{
-Ref. [1] hus been invalubble to the preparation of this thesis.

t the diecowery of the nucleum[5] was stllf 15 years hence
} 
the weak interaction maximally violates [arity[y], which led (after some confusion) to the $V-A$ theory of the weak interaction[0] with the four-fermion interaction baed on mediating charged vector boons, denoted $W^{ \pm}$.

Of course, the development of weak interaction theory did not proceed independently of the growing population of elementary particlex. Studks of $\mu$ decay in the 1940's indeated that the weak conpling was similar in magnitude and gtructure to that obecrved in $\beta$ decay, which hed many to postulate that the neak interaction wan universal. As the quest for unification continued, the electromagnetic interaction also came uuder scrutiny, and the searcl for u coublued theory of the weak and electromagnetic interactions was begun. The earlier introduction by Yang and Mills of a non-abelian theory of particle interactions[10] provided the besls, and after a decade or mote of casting about for the correct fonnulation of the theory, Weimberg[11] and Sulam[12] proposed a theory of the electroweak interaction which contained the underlying symmetry $S U(2) \times U(1)[13]$. The mification of the two theories necessitated the introduction of a new gauge boson, the $Z^{0}$, which is the weak interaction analogue to the photon. The Higgs method[14] of spont ancously breaking the symmetry of the theory[15] was used to trantform the massless gange bosous which result from the Yang-Mills theory into the massless photon and the massive $Z^{0}$ and $W^{ \pm}$. One coential difficulty remeinged, homever: this thoory setuned not to be renormalizable. This impasse wos solved by 't Hooft[16] in 1971, utd the theory was extially complete.

- It retnained for the experimentalists to find some evidence of the weak neutra] current or the massive gauge bosons themselves. This first cane in atudies of neutrino scutteritis, where the elastic sattering[17] $\nu_{\mu} e \rightarrow \nu_{\mu} E$ and the inelastic scattering[18] $\nu_{\mu} N \rightarrow \nu_{\mu}+X$ were seen. Corroborating evidence for the doublet structure of $S U(2) \times U(1)$ was provideel in the following ycar by the discovery of the charin quark[19]. The apparent symmetry between the generations of quarks and leptons whs upset shortly thereafter by the obserwition of anuther heavy lepton, the $\tau[20]$. The discovery of the $\boldsymbol{\Upsilon}$ resonances in $\boldsymbol{\mu}^{+} \boldsymbol{\mu}^{-}$production[21] provided the first quark of the third gencration, and reestablished the symmetry of the electroweak theory. Froin this point, the weurch way on for the clusive top quark, the last member of the third genoration. Eventually, the $W^{ \pm}$and $Z^{0}$ bowons were viwerved directly in $p p$ 
collivions[22] in 1962 ond 1983, providiag the fital piece of material evideace that the Weinbeis-Salam model of the electroweak interaction properly dexcribes Nature. The recent observation of the long-sought top quark[23] provides the final piece of the particle complement puzzle.

As it stands, what has cone to be called the Standard Model of electroweak interections is a remarkably succesful theory. Stuce its initial formulation, virtully all of its predictions have been verified to ever-increasing precision. Attention has now turned from confirmation of the model's validity to the questions underlying the assumptions involved in its construction: why are there three generatikns of fermione? What is the relationship between quarks and lejtons, given that both of then are necessary for the theory to be renormalizable[24]? Whiat is the nature of $C P$ violation? Just what is the true Higgs mechanisn? The current program of precision messurements of clectroweak parameters was undertaken to provide clues to the answers of these questions. Hopefully, the answers will be [orthcoming.

\subsection{The Electroweak Standard Model}

In this section, we present an overvicw of the components of the electroweak theory described in the Standard Model'.

The unification of the clectromagnctic and weak foress is accomplished by modifying the eletromagnetic current

$$
-i e j_{\mu}^{e n} \dot{w}^{\mu}=-i c\left(\bar{\psi} \gamma_{\mu} Q \psi\right) A^{\mu}
$$

to include the weak interaction. Here, $A^{\prime \prime}$ is the four-potenthal of the electromagoetic ficld, statisfying $\partial^{2} A=0, \psi$ is the fermion spisor wuvefunction, $\gamma_{\mu}$ is the usul spinor matrix, and $\mathcal{Q}$ is the charge operator, which has cigenvalus -1 for tbe electron. To complete this unification, the electromagnetic current is replased by two new currents: an $S U(2)_{L}$ isotriplet of wrak currents $\mathbf{J}_{\mu}$ which couple to three vector busous $\mathbf{W}^{\mu}$, given by

$$
-i g J_{\mu} \cdot W^{\mu}=-i g \bar{X}_{L} \gamma_{\mu} T \cdot W^{\mu} \chi_{L}
$$

\footnotetext{
"More detalied exponklons of this type cen bo found in, e.g., Rer. [25] or Rer. [26].
} 
Tubie 2.1: The $L$ ixodoublets and $R$ isosinglets of $S U(2)_{L} \times U(1)_{Y}$

\section{Quarks}
$(d)_{L} \quad(u)_{n}$ or $(d)_{R}$
$\left(\begin{array}{l}c \\ b\end{array}\right)_{L}$
$(c)_{R}$ or $(b)_{R}$
$\left(\begin{array}{l}t \\ b\end{array}\right)_{L}$
$(t)_{R}$ or $(b)_{R}$

\section{Leptons}
$\left(\begin{array}{l}\omega_{\mathrm{e}} \\ )_{2}\end{array}\right.$
(e) $\boldsymbol{H}$
$\left(\begin{array}{c}\nu_{k} \\ \mu\end{array}\right)_{L}$
$(\mu)_{\mathrm{A}}$
$\left(\begin{array}{l}\nu_{r} \\ r\end{array}\right)_{L}$
$(\tau)_{A}$

and a $U(1)$ weak hypercharge current $j_{\mu}^{Y}$ coupled to a fourth vector boson $B^{\mu}$, given by

$$
-i \frac{g^{\prime}}{2} j_{\mu}^{Y} B^{\mu}=-i g^{\prime} \tilde{\psi} \gamma_{\mu} \frac{Y}{2} \psi B^{*} .
$$

The operators $\mathbf{T}$ and $Y$ are the gonerators of the $S U(2)_{L}$ and $U(1)_{Y}$ gauge groups; the operators $T$ satisfy the commutstion relation

$$
\left[T^{1}, T^{j}\right]=i E_{i j k} T^{*}
$$

The new coupling constants of the theory are $g$ and $g^{\prime}$. The fundamental fermions have been arranged in left-handed isodoublets $\chi_{L}$ and right-handed iscoinglets $\psi_{R}$, which trarsform as

$$
\begin{aligned}
& \chi_{L} \rightarrow \chi_{L}^{\prime}=e^{i \alpha(x) \cdot T+i \beta(x) y} \chi t \\
& \psi_{R} \rightarrow \psi_{R}^{\prime}=e^{i \beta(x) \psi_{\psi}} \psi_{R}
\end{aligned}
$$

under the infinitesimal guuge trangformations given by $\alpha(x)$ and $\beta(x)$. The grouping of the fermions is shown in Table 2.1. Given a field corresponding to any fermion $f$, tlse left-handed and right-handed components can be projected out by meany of the operstor $\gamma_{\mathrm{s}}$ :

$$
f_{R}=\frac{1}{2}\left(1-\gamma_{b}\right) f, \quad f_{L}=\frac{1}{2}\left(1+\gamma_{B}\right) f .
$$


The generul electroweak interuction

$$
-i g\left(J^{\top}\right)^{\mu} W_{\mu}^{i}-\frac{g^{\prime}}{2}\left(j^{\gamma}\right)^{*} B_{\mu}
$$

can be rewritten in terms of the clectric-charge raising and lowering bosons

$$
W_{\mu}^{ \pm}=\sqrt{\frac{1}{2}}\left(W_{\mu}^{1} \mp W_{\mu}^{2}\right)
$$

which only couple to the left-handed fermion doublets, and a linear combination of two neutral bosons $A^{\mu}$ and $Z^{\mu}$

$$
\begin{aligned}
-i g\left(J^{3}\right)_{\mu}\left(W^{3}\right)^{\mu}-\frac{g^{\prime}}{2}\left(j^{\gamma}\right)^{\mu} B_{\mu}= & -i\left(g \sin \theta_{W} J_{\mu}^{3}+g^{\prime} \cos \theta_{W} \frac{j_{\mu}^{\gamma}}{2}\right) A^{\mu} \\
& -i\left(g \cos \theta_{W} J_{\mu}^{3}-g^{\prime} \sin \theta_{W} \frac{j_{\mu}^{\gamma}}{2}\right) Z^{\mu} .
\end{aligned}
$$

This tinear combination is juse the fields $W^{3}$ and $B$ rotated by an angle $\theta_{W}$ in the $\left(W^{3}, B\right)$ plane. The value of the so-ralled Weinberg angle $\theta_{W}$ is not predicted by the theory and must be determined from experiment. Relutions between the coupling $g$ and $g^{\prime}$ and $\theta_{W}$ can be derived by noting that the generators of the two groups aloo satiafy the relation

$$
Q=T^{3}+\frac{Y}{2},
$$

where $Q$ is the electric charge. This leads to an expression of the electromagnetic current in terms of the two newly introduced weak currents:

$$
j_{\mu}^{e m}=J_{\mu}^{3}+\frac{1}{2} j_{\mu}^{Y} .
$$

This choice of currents, with the electric charge given by Eq. 2.10, guarantes that the gauge boson $A_{\mu}$ which mediates the clectromagnetic interaction will remain maseless after the spontaneous symmetry breaking of the Higgs mechamism (to be discueed in the next section) that gives mases to the other gauge bosons.

If we identify the firat term on the right side of Eq. 2.9 with the electromegnetic current, we sec imnediately that

$$
g \sin \theta_{W}=g^{\prime} \cos \theta_{W^{\prime}}=e .
$$


II addition, the relationship

$$
\tan \theta_{w}=\frac{g^{\prime}}{g}
$$

also boths. We can now rewrite the second term on the right hand side of $\mathrm{Eq} .2 .9$ as

$$
-i \frac{g}{\cos \theta_{W}}\left(J_{\mu}^{3}-\sin ^{2} \theta_{\mu} \cdot j_{\mu}^{f m}\right) Z^{\mu} \equiv-i \frac{g}{\cos \theta_{W}} J_{\mu}^{N C} Z^{\mu} .
$$

The definition

$$
J_{\mu}^{N G}=J_{\mu}^{3}-\sin ^{2} \theta_{\omega} j_{\mu}^{\mathrm{em}}
$$

completes the spexification of the two oberved neutral interactions in terms of the new currents $\mathbf{J}$ and $j^{\gamma}$ that were introduced, Thus, the $S U(2)_{L} \times U(1)_{Y}$ formulation of the electroweak theory does contain both the electrc magnetic and weak forces with their approprinte properties. The only inisying element is the racans to arrive at the lnuge mass splitting berwen the ploton and the weak vector bosons. This is provided by the Higgt unecluarisin, the topic of the next section.

\subsubsection{Spontanecus Symmetry Breaking:}

\section{The Higgs Mechanism}

As the demands of gauge invariance und renornalizability prolibit the introduction of such terms as $M K_{W}, W_{\mu} W^{\mu}$ into the theory, ancther mechanisn must be invoiked that preserves the desirexi aspects of the theory. This is provided by introducing a term in the electrowed lagrangiant of the form

$$
\mathcal{L}=\left|i\left(\partial_{\mu}-g \mathbf{T} \cdot \mathbf{W}_{\mu}-\frac{g^{\prime}}{2} B_{\mu}\right) \phi\right|^{2}-V(\phi),
$$

where there are four sealar fields $\phi_{i}$ and the potential $v(\phi)$ is given by

$$
V(\phi)=\mu^{2} \phi^{\dagger} \phi+\lambda\left(\phi^{\dagger} \phi\right)^{2} .
$$

To preserve the gmuge invariance of the lagrungian, the fields $\phi$ must be members of $S U(2) \times U(I)$ multiplets. The least complicatexi choice is that of $\_Y=1$ igodoublet, which is given by

$$
\phi=\left(\begin{array}{l}
\left(\phi_{1}+i \phi_{1}\right) / \sqrt{2} \\
\left(\phi_{3}+i \phi_{4}\right) / \sqrt{2}
\end{array}\right) .
$$


If we choose the parameterg $\mu^{2}<0$ and $\lambda>0$, thu potential $V(\phi)$ posecese an SU(2)-invariant ununifold of minime whowe locus is given by

$$
\phi^{\dagger} \phi=\frac{1}{2}\left(\phi_{1}^{2}+\phi_{2}^{2}+\phi_{1}^{2}+\phi_{4}^{2}\right)=-\frac{\mu^{2}}{2 \lambda}=\frac{1}{2} v^{3} .
$$

We can now "spontaweusaly break" the $S U(2)$ sywumetry by asuuning that the phyo ical vacuum is given by some specific choke of the minimum of $V(\phi), e, g$.

$$
\phi_{1}=\phi_{2}=\phi_{1}=0, \quad \phi_{3}^{2}=-\frac{\mu^{2}}{\lambda}=v^{2} .
$$

The vacuum $\phi_{0}$ is then polven (from Eq. 2.18) by

$$
\phi=\sqrt{\frac{1}{2}}\left(\begin{array}{l}
0 \\
v
\end{array}\right) \text {. }
$$

This choice of vactum ts not SU(2) invariant, but the lagrangian of En. 2.16 still is, as it does not eare where the minimugn in the vecuum is located.

To examine the consequences of this choice of vacuum, we cau corsides small oncillations of the fhelds about this minimum. These fluctuations can be paraunetiaced by the four real fieldy $\theta_{1}, \theta_{2}, \theta_{1}$, and $h$ uning the form

$$
\phi(x)=\frac{e^{\pi \cdot \bar{\varphi}(x) / v}}{\sqrt{2}}\left(\begin{array}{c}
0 \\
v+h(x)
\end{array}\right) .
$$

Substitution of this forn into Eq 2.16 results in three massless fields ${ }^{5} \theta_{i}$ a term proportional to $2 \mu^{2} h^{2}$, and a number of cross terms proportional to $\left(W^{i}\right)_{\mu} \partial^{\mu} \theta_{j}$. We are free to exploit the $S U(2)$ gauge symmetry by performing a gauge transformation

$$
\phi \rightarrow \phi^{\prime}=e^{-\left[r+(x) / \omega^{\prime} \phi\right.}
$$

whicls leaves us with the field

$$
\phi(x)=\left(\begin{array}{c}
0 \\
\frac{v+4 \sqrt{x})}{\sqrt{2}}
\end{array}\right) .
$$

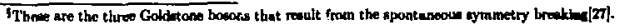


Upon inserting this parametrization into the lagranginu (2.t6), we find that that the flekd $h$, which we now call the Higges perticle, has acquired a mase $-\frac{\lambda p^{2}}{i}$, Hnd the gauge booons $W^{i}$ have acquired masses via the term

$$
\left|\left(-i g \mathbf{T} \cdot \mathbf{W}_{\mu}-i \frac{g^{\prime}}{2} B_{\mu}\right) \phi\right|^{2}
$$

which, after aquaring and soine algebra, yields

$$
\left(\frac{1}{2} v g\right)^{2} W_{\mu}^{+} W-\mu+\frac{1}{8} v^{2}\left[g W_{\mu}^{3}-g^{\prime} B_{\mu}\right]^{2}+0\left[g^{\prime} W_{\mu}^{3}+g B_{\mu}\right]^{2} \text {. }
$$

The mases of the tharged aud nettral boens can be read of from this expression by identifying the coufficionts of the various terms witl: $M_{W}^{2} W+W-, \frac{1}{2} M_{Z}^{2} Z^{2}$ and $\frac{1}{2} M_{A}^{2} A^{2}$, respectively. This gives

$$
M_{i v}=\frac{1}{2} v g,
$$

and, upon normalization of the fields,

$$
\begin{array}{lll}
A_{\nu}=\frac{g^{\prime} W_{\mu}^{\prime 3}+g B_{\mu}}{\sqrt{g^{2}+g^{3}}} & \text { with } & M_{A}=0 \\
Z_{\mu}=\frac{g W_{\mu}^{3}-g^{\prime} B_{\mu}}{\sqrt{g^{2}+g^{\prime 2}}} & \text { with } & M_{z}=\frac{1}{2} \sqrt{g^{2}+g^{\prime 2}} .
\end{array}
$$

Thus, we have arrived at a theory which contains three an msive and one messless vector boson. Using the information from $\mathrm{Eq} .2 .12$, we see that the Higgs mechenism has given us a new reintionship between the $W^{\prime}$ and $Z$ nurgass:

$$
\frac{M_{1 W}}{M_{Z}}=\frac{g}{\sqrt{g^{2}+g^{M}}}=\cos O_{W} .
$$

This prediction can be tested experinentally to verify that the Higes mechanism is responsible for the symmetry breaking of the clectriweak thcory.

\subsection{Specifying the Standard Model}

As we have just seen, the three parameters $g$ (the $S U(2)$ coupling constant), $g^{\prime}$ (the $U(1)$ coupling constant), and $\left\langle p h t_{0}\right\rangle$ or expivatenty, w (the Higgo vacuun expectation 
value) stifice to specify all of the tree level piocses that occir in the standard inodel. However, nons of thesc parandeters are experimentally acceatible. Inatead, we can define three combinations of tixem which can be menoured experimentaly. Firs, from $E$ q. 2,12, we bave

$$
\alpha \equiv \frac{e^{2}}{4 \pi}=\frac{y g^{\prime 2}}{4 \pi \sqrt{g^{2}+g^{\prime 2}}} .
$$

Next, we take frust the lew eucryy Leluaviour of the weak interaction the Fermi constant $G_{F}$, which is given by the charged weak current at $q^{2} \rightarrow 0$. In this case, wo can rewrite the Jagrangiun as

$$
\mathcal{L}_{C C}=-i \frac{g}{\sqrt{2}}\left(J^{\mu} W_{\nu}^{+}+J^{\mu} W_{\mu}^{-}\right) .
$$

In the low $q^{2}$ limit, this leads to the Ferıni four-point anplitude

$$
M_{C C}=\left(\frac{g}{\sqrt{2}} J_{\mu}\right)\left(\frac{1}{\Lambda_{W W}^{2}}\right)\left(\frac{g}{\sqrt{2}} j^{j \dagger}\right) \equiv \frac{4 C_{r}}{\sqrt{2}} J_{\mu} J^{\mu \dagger},
$$

whence we can extruct the relption

$$
G_{F}=\frac{g^{2}}{4 \sqrt{2} M_{\|^{2}}^{2}}=\frac{1}{2 \sqrt{2}\left(\phi_{0}\right)^{2}} .
$$

Finally, wo have

$$
M_{Z}=\frac{1}{\sqrt{2}}(\phi \nu) \sqrt{g^{2}+g^{2}} .
$$

The volues of these parameters are slown in Table 2.2 Any other measurable combiuntion of these thre parancters, such as

$$
\sin ^{2} \theta_{H^{\prime}}=\frac{g^{2}}{y^{2}+g^{22}},
$$

can be eompared agaitst its predicted value using the ahove tlu ree parwneters to test thic Standihrd Modr!.

At higher order, of course, the unspecificd fermion muskey utu Higgs mass and couplings cuter into the theoretical iredictions in the torm of rimlineive corrections. This hus the effect of modifying the observibles in such a way as to slow experimental access, given the Standard Model caleulntions ks input, to the n-yet-unoberved Higgs, and allows the verifiention of the higher-order behaviour of the theory (or, hopefully, to mone violation of the theoretical predictions?). 
Thble 2.2: The paraineters which spexily the clectroweak Standerd Model at tree level

\begin{tabular}{|c|c|c|}
\hline Parameter & Measured Valuc & Precision \\
\hline $\begin{array}{l}a \\
G_{z}\end{array}$ & $\begin{array}{c}1 / 137.0359895(61) \\
1.16637(2) \times 10^{-3}(\mathrm{GeV})^{-2} \\
91.187(7) \mathrm{GeV} / \mathrm{c}^{2} \\
{[30]}\end{array}$ & $\begin{array}{l}4.5 \times 10^{-6} \\
1.7 \times 10^{-3} \\
7.7 \times 10^{-5}\end{array}$ \\
\hline
\end{tabular}

\section{3 $Z^{0}$ Production and Decay}

Since 1989, we lave had direct access to the weak interactions by studying the production and decay of $Z^{0}$ basons in the pristine envirument of $\mathrm{e}^{+} \mathrm{c}^{-*}$ collisions. Since the $Z^{0}$ couples to dil known formions, the detailed vertex structure of the electroweak interaction can be studierl through measuring the $Z^{0}$ coupling to the initial and final state particles.

To aid in our discussion of physics at the $Z^{0}$ pole, can rewtite the weak neutral current

$$
-i \frac{g}{\cos \theta_{w}}\left(J_{\mu}^{3}-\sin ^{2} \theta_{w} j_{\mu}^{\mathrm{em}}\right) Z^{\mu}
$$

in teris of the quantum numbers of the electroweak interaction, $T^{3}$ and $Q$ (cf. Eq. 2.10)

$$
-i \frac{g}{\cos \theta_{\mu^{*}}} \pi_{\gamma} \gamma^{\mu}\left[\frac{1}{2}\left(1-\gamma^{8}\right) T_{f}^{3}-\sin ^{2} \theta_{W} Q_{f}\right] \psi_{J} Z_{\mu} \text {. }
$$

We call express the vertex factor in terus of the vector $\left(v_{f}\right)$ and axial vector $\left(a_{f}\right)$ couplings to the $Z^{0}$

$$
-i \cos _{w}^{g} \gamma^{\mu} \frac{1}{2}\left(v_{J}-a_{j} \gamma^{s}\right)
$$

where

$$
\begin{aligned}
& v_{f}=T_{f}^{3}-2 \sin ^{2} \theta_{W} Q_{f} \\
& a_{l}=T_{l}^{3} .
\end{aligned}
$$

Here, $Q_{J}$ and $T_{J}$ are the tharge and the third componeat of weak isaspin for the fermion $f$. For completences, the walues of the vector and axial vector couplings for each of the fermions is listed in Table 2,3. 
Table 2.3: The vector and exial vector conplings for the fermions to the $Z^{0}$.

\begin{tabular}{|c|cc|}
\hline Fermition & $a_{f}$ & $v_{f}$ \\
\hline$\nu_{e}, \nu_{p+}, \nu_{r}$ & $\frac{1}{2}$ & $\frac{1}{2}$ \\
$e, \mu, r$ & $-\frac{1}{2}$ & $-\frac{1}{2}+2 \sin ^{2} \theta_{W}$ \\
$u_{,} c_{k} t$ & $\frac{1}{2}$ & $\frac{1}{2}-\frac{1}{3} \sin ^{2} \theta_{w}$ \\
$d, s, b$ & $-\frac{1}{2}$ & $-\frac{1}{2}+\frac{2}{3} \sin ^{2} \theta_{w}$ \\
\hline
\end{tabular}

In Figure 2.1, we shown the tota' cross section for $e^{+} e^{-} \rightarrow \mu^{+} \mu^{-}$. The large resonance arourul the $Z^{0}$ polc is quite obvinus. Since it dominates the photon-mediated procesees by a factor of $\sim 800$, we coul safoly neglect the effects of the electromasnetic interaction when discussing processes that accur at the $Z^{a}$ pote. In arlition, at the pole the effects of $\gamma-Z^{0}$ interference vanish or become negligible, so we are left to consider the elcctroweak current as consisting of pure $Z^{0}$ exchange. As will be discussed in Chapter 4, the SLC is capabie of protucing a longitudinally polarined electron beam, which, as me shall sec montenturily, allows us to study the chiral. structure of the electronenk interaction in unore detnil than proviously possible.

The differential crass settion for $e^{+} c^{-} \rightarrow / \bar{f}$ at the $Z^{0}$ pole can be expresed as[31]

$$
\begin{aligned}
& \left.\frac{d \sigma}{d \Omega}\right|_{Z}=\frac{a^{2}}{4 \sin ^{4} 2 \theta_{W}} \cdot \frac{s}{\left(s-M_{z}^{2}\right)^{2}+\Gamma_{z} s^{2} / M_{z}^{2}} . \\
& {\left[\left(1+\cos ^{2} \theta\right)\left(u_{e}^{2}+a_{\varepsilon}^{2}\right)-y \cos \theta u_{r} a_{\mathrm{r}}: u_{j} u_{f}\right]} \\
& \text { - } P_{s}^{-}\left[2\left(1+\cos ^{2} \theta\right) v_{r} v_{r}\left(v_{j}^{2}+a_{j}^{2}\right)+4 \cos \theta\left(v_{r}^{2}+a_{r}^{2}\right) v_{j} a_{f}\right]
\end{aligned}
$$

Here, $P_{z}^{-}$is the longitudinal polarization of the efittron brun, where $P_{z}^{-}=1(-1)$ corresponds to right- (leit-) handed clectrons, $s$ is the squure of the center-of-mass collision chergy, and the winth of the $Z^{0}$ resonance is sp rified by $\Gamma_{z 2}$. The angle $\theta$ is the polar angle of the outgoing fermion with mipect to lwe electron bean direction. We have neglected in this representation extra terme that could arise if the poeitron beam is polarized, and tertn that wre due to transverse besm polarization, since neither apply i.. our particular case. Upon inspertiosi of this formule, it can be 


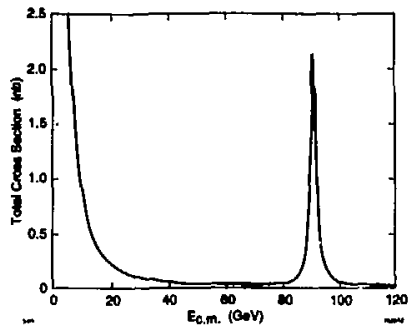

Figure 2.1: The total cross section for $c^{+} e^{-} \rightarrow \mu^{+} \mu^{-}$vs, ceutcr-of-uass energy

seen that hoth the polarization dependent and the polarization-independent parts of this expression contuin polar-angle anti-synmetric ternts, which implies that all $Z^{0}$ decays will exhibit fernion production asymmetries. As we sliall see below, these are enhaneed for the polarized case.

In general, thw effects of $\gamma-Z$ Interference cun be included by adding the aupplementary cross section

$$
\begin{aligned}
\left.\frac{d \sigma}{\mathrm{\alpha}}\right|_{\Upsilon Z}= & -2 Q_{J}\left(1-\frac{M_{Z}^{2}}{s}\right) \frac{.}{\left(s-\Lambda_{Z}^{2}\right)^{2}+\Gamma_{Z}^{2} \theta^{2} / M_{Z}^{2}} \\
& \left\{\left[\left(1+\cos ^{2} \theta\right) v_{\mathrm{r}} v_{J}+2 \cos \theta_{\varepsilon} a_{J}\right]-P_{z}^{-}\left[\left(1+\cos ^{2} \theta_{1} a_{\mathrm{e}} v_{f}+2 \cos \theta v_{\mathrm{q}} a_{J}\right]\right\} .\right.
\end{aligned}
$$

As mentioned above, this contrihution sanishes at the pole, where $M_{2}^{2}=s$. Since the energy dependasce of this crues ection is different from that of the pure $Z^{0}$ exchesige, however, the total fermion production cross setion and any fermion production nzymmetries Jepend on the center-ol-naws energy. In considering these cffects on the funl statc fermion production asymmetrics, we will see that eliey are omall. 


\subsection{Renormalization of the Electroweak Coupling}

In this mection, we consider the rodiative correction to the electrowealk coupling by exanining the higher-order diagramu that modify the tree-level coupling. A gener. discusaion of electroweak radiative corrections can be found in Rei. [32]. We divide the discuswion of the virtual corrections an in Ref. [32] into "wif" corrections, which can

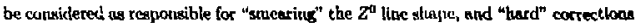
which determine the higher-order "unsnereaced" resonance through the renormalization of the electroweak parametcrs. Since a proper expoxition of the renormalization of the full electroweuk theory would be toro lengthy, we prosent merely a sketch of the procedure here.

\subsection{Soft Radiative Corrections}

The predomnant soft radiative correctisns oceur to $\mathcal{O}(\alpha)$ and are due primarily to initial atate radiation (ISR). The diagruuss that contribute to ISR at the $Z^{0}$ pole wre shown in Figure 2.2. Their effect is to modily the shape of the $Z^{a}$ resonsuce as a function of center-of-mass enerby. The rclative sigu of thls effect in ensily understood: since ISR is a relatively prolsably occurnice, some fraction of collisions that occur when the colliding beans are at the $Z^{\prime \prime}$ pole will take place at a reduced center-ofmass energy, where the $Z^{0}$ production cross section is lower. Thus, the peak cross section is reduced by initisl stnte radiution. In uddition, those collisions which occur

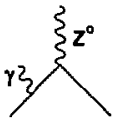

3)

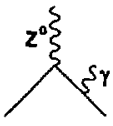

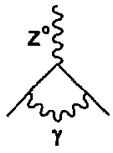

MA10

Figure 2.2: Tlic Icurlitug-order initiel state QED cortectious to the $Z^{0}$ resonance shape. 


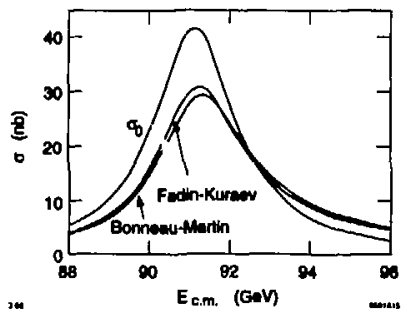

Figure 2.3: Variution of the $Z^{\prime \prime}$ resonence shape from ISR (nec text).

at a center-of--1nuse energy above the $Z^{0}$ pole can radiate down to the pole, where the crow-section is larger. This results in m overall upwerst shift of the obeerved pesk croos section, as well as an asynunetry in the shape of the resonance, with the high energy side bejug larger thun the low entergy side'. A comparison of the treelesel cross section with two higher-order calculations is shown in Figure 2.3. The Bonneau-Martin culculntion[33] is a fixed-ordex calculation per[ormed at $O(a)$. The Fadin-Kursev result [i4] is obtained by cvolving the radiating bean particles down to a lower ene ny by a proces simihir to the Altarelli-Parisi equation of QCD" and summing th: rontributions of the leating terns in the spliting kernel to O $\left(a^{2}\right)$. One can see that the reduction in the peak crow section is quite large, $\sim 30 \%$; contideration of these effecty is crucial in makitug precision electroweak messurements at the $Z^{0}$ pole,

\footnotetext{
TThe $c^{+} c^{-}$palt can always tadiate doun to the $Z^{0}$ proak.

ISee Sextion 3.1.4.
} 


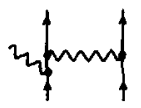

(1)

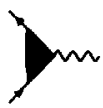

(b)

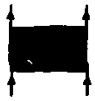

(c)

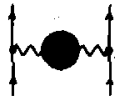

(d)

Figure 2.4: A wehematic view of the different electroweak radintive correctious, including (a) initial and final state breinentruhlung, (b) vertex corrections, (c) box diagrams, and (d) self-energy ("oblique") corrections.

\subsubsection{Hard Radlative Corrections}

A complete consideration of the radiative corrections to the ehrtromeak lagrangian should inclube the elfcets of all of the diugranus show'n schemuntically in Figure 2.4. The important effects of the initial-stute bremestralylung gripliss of Fig. 2.40 were discused in the previous section. We turn sow to ful overview of the other corrections which modify the electrowenk parameters. The nout importnnt of these to our present discussion are the oblikiwe correctiolss of Fig. 2.4d, which provide the primary contributions to the renomalized coughings and ure coutnan to all electromeak interactious. The vertex corrections (Fig. 2.4b) are proccsisnpecific in the sense that they depend on the masses and flavory of the external fermions. The box cometions (Fig. 2.4c) ara in most cases negligible[35] urd will not be considured further. We will follow the totation of Hollik[36] and Bordin[37] while kecping in tnind the organizational simplicity of Kennely and Ly'mi[38].

\section{Renormalization of the Electromagnetic Coupling}

As an example of the renormalization jrocedure, we consider finst the renormalization of the electromagnetic coupling $a$. By gunge invariance", the only contribution to the renormalization of the electric charge conse from the photon melf-energy diagrems,

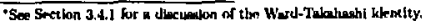




$$
\operatorname{mon}=10^{2} \pi_{\infty}\left(q^{2}\right)\left(g^{\mu \nu}-\frac{q^{\mu} q^{\nu}}{q^{2}}\right)
$$

(a)

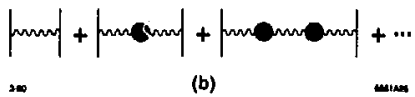

Figure 2.5: A schematic view of (a) the photon self-cnergy correction and the definjtion of the vertex factors and (b) the infinite series of one-particle irreducible diagranis that contrit te to the remormalization of the electric charge.

shown schetnatically in Figure 2.5. It is tonvenient to define the function

$$
\Pi_{G Q}^{\prime}\left(q^{2}\right) \equiv \frac{\Pi_{\infty Q}}{q^{2}},
$$

where II $Q Q$ is the coefficient of $g^{\mu v}$ in the photon self-energy in Fig. 2.5a. If the onepartick irruducible diagrams of Fig. 2.5b ure summed to all orders[39], one obtainy

$$
\frac{-i e_{0}^{2}}{q^{2}}\left(1+c_{0}^{2} n_{Q Q} \frac{1}{q^{2}}+\ldots\right) g^{\mu \nu}=-\frac{i q^{\nu}}{\varphi^{2}} \cdot \frac{e_{0}^{2}}{1-e_{0}^{2} n_{\phi Q}^{\prime}}
$$

20 the expression Ior the complete photon propagator, where $e_{0}$ is the bare electric charge. This suggests the definition of an effective charge $e_{e f f} \equiv \mathfrak{e}$ given by

$$
e\left(q^{2}\right)=\frac{e_{0}^{2}}{1-e_{0}^{2} \pi_{Q Q}^{\prime}},
$$

where now the coupling $e$ depends on the $q^{2}$ of the internction, i.e., it "runs". The value of $z$ at $q^{2}=0$ is choeen as the reference coupling: this can be determined from the $g-2$ of the electron or from orher measurcuneuts such as the Josephson effect. This allows us to define the familiar quantity $a$ by $4 \pi \sigma=e^{2}\left(q^{2}=0\right)$, where the value of $\alpha$ from this definition is given in Table 2.2. From this definition, the running of $\alpha$ is given by

$$
\frac{1}{4 \pi a\left(q^{2}\right)}=\frac{1}{4 \pi \alpha}-\left[\Pi_{Q \phi}^{\prime}\left(q^{2}\right)-n_{Q \phi}^{\prime}(0)\right] .
$$


Thangl: the quuntity $\mathrm{n}_{Q Q}^{\prime}$ diverges, the difference $\mathrm{n}_{Q Q}^{\prime}\left(\tau^{2}\right)-\Pi_{C O}^{\prime}(0)$ is finite. Of coturse, all of the difficultive involved in renormalizing t) uetually calculating the quant ity $\mathrm{I}_{Q Q Q}{ }_{\text {Q }}$ in a convenient gauge. We will onit a diecursion of that here, and merely mention that the value of $\alpha\left(M_{Z}^{2}\right)$ is necesary for the compruison of expcrimentul rexults obtained at the $Z^{0}$ pole with theoretical predlclions. This quatitity can be obtained from existing measurements of $e^{+} e^{-} \rightarrow f \bar{f}$ by a theoretical argument[40] using the optical theoren to relate the hadronic corrections to formard Bhabha seattering to the total cross section for $e^{+} e^{-} \rightarrow$ hadrons. A recent. result of this procedure is [41]

$$
\mathrm{a}^{-\mathrm{t}}\left(M_{Z}^{2}\right)=129.08 \pm 0.10,
$$

where the dominart uncertuinty is frou tive inpreise measurements of the total croes section for $\mathrm{e}^{+} \mathrm{e}^{-} \rightarrow$ hidrons at low-energy ( $2 \mathrm{GeV}<E_{\text {CM }}<3.0 \mathrm{GeV}$ ).

\section{Renormulizstion of the Weak Coupling}

Bechuse of the number of ditferent diagrans involved, a discusion of the renornalization of the weuk couplings becornes quite complicuted. As a summiary of the types of diagrams involved, wo shew in Figures 2.6 and 2.7 some of the contributing procenses. Their effect on the couplings is most easily cxpressed in terms of the induced modification to the form of the neutral curent vertex ${ }^{\dagger}$. If we rewrite the weak neutral eurent in terms the parameters chosen to specify the theory, we obtain

$$
-i M_{Z}\left[4 \sqrt{2} G_{F}\right]^{\frac{1}{2}} \gamma^{\mu}\left[\frac{1}{2}\left(1-\gamma^{3}\right) T_{J}^{J}-\left.\sin ^{2} \theta_{W}\right|_{s} Q_{J}\right] \text {. }
$$

where, for notational convenience, we have :sect the Sirlin definition of $\sin ^{2} \theta_{w}[42]$ :

$$
\left.\sin ^{2} \theta_{u} \cdot\right|_{S} \equiv 1-\frac{M_{U^{\prime}}^{2}}{M_{2}^{2}}
$$

which elevates the tree-level relation of Ex. 2.30 to a definition which will be taken us valid to all orders in perturbation theory.

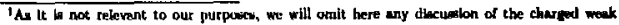
current.
} 


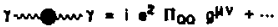

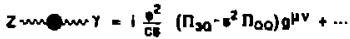

$$
\begin{aligned}
& z \operatorname{manv} 2=i \frac{\varepsilon^{2}}{c^{2} v^{2}}\left(\Pi_{3 j}-2 u^{2} n_{30}+s^{4} n_{\infty}\right) q^{\mu v}+\ldots \\
& \text { Wrangumw }=i \frac{l^{2}}{g^{2}} n_{11} g^{\mu v}+\ldots
\end{aligned}
$$

Figure 2.ti: A schematic view of self-energy diagrams that oceur in calculating reilative corrections to the wenk interaction und the definition of the vertex factors inuolved. Here, $s^{2}\left(c^{2}\right)=\sin ^{2} \theta_{w}\left(\cos ^{2} \theta_{w}\right)$.
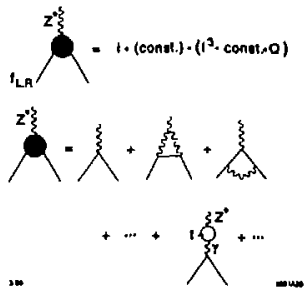

Figure 2.7: A schematic vew of self-energy dieprams that occur in calculating radiative conections to the weal interaction and the definition of the vertex factors involved. Here, $s^{2}\left(c^{2}\right)=\sin ^{2} \theta_{W}\left(\cos ^{2} \theta_{W}\right)$. 
Whien the efiects of tive one-loop corrections are included, the form of the current from Eq. z.45 can be retained with the addition of an overall normalization factor p/ and an Bdditional vertex form factor $\kappa_{f}$, where the cturrent is now given bys

$$
-i M_{Z}\left[4 \sqrt{2} G_{F} \rho_{f}\right]^{1} \tau^{\mu}\left[\frac{1}{2}\left(1-\gamma^{3}\right) T_{j}^{3}-\kappa_{l} \sin ^{2} \theta_{w} \mid ; Q_{l}\right] \text {. }
$$

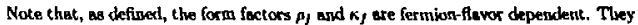
can, however, be sepurntid into univenal parts (due to tise boeon seli-energies) and nou-univeral parts (from the vertex corrections and self-unergies of the external fermion lines) as follows:

$$
\begin{aligned}
& \rho_{j}=1+(\Delta \rho)_{\text {univ }}+(\Delta \rho)_{\text {nom-univ }} \\
& k_{j}=1+(\Delta \kappa)_{\text {miv }}+(\Delta k)_{\text {nom-univ }} .
\end{aligned}
$$

The universal parts of the form factors contain csantially all of the the dependence of the couplings on the top quark tnaw $\left(m_{l}\right)$ and Higgs mas, except in the case of $b$ ¿quarks, where large vertex corrections are inuportant. In general, with the preceding excoption, the univirsal portions of the fonn factors are much larger than the uchuniversal contributions. To lowest order, we have

$$
\begin{aligned}
1+(\Delta \rho)_{\text {univ }} & =\frac{1}{1-\overline{\Delta \bar{\rho}}}+\ldots \\
(\Delta \kappa)_{\text {wiv }} & =\frac{\left.\cos ^{2} \theta_{u}\right|_{s}}{\left.\operatorname{sid}^{2} \theta_{u}\right|_{s}} \Delta \bar{\rho},
\end{aligned}
$$

where[43]

$$
\Delta \rho=N_{C} \frac{G_{F} m_{l}^{2}}{8 \pi^{2} \sqrt{2}}\left[1+\frac{G_{r} m_{l}^{2}}{8 \pi^{2} \sqrt{2}}\left(19-2 \pi^{2}\right)\right]
$$

is the result of cakculating the correction inclukling 2-loop irreducible diagrams.

The form of Equation 2.50 suggests that we define an "aflective" renormalized version of $\sin ^{2} \theta_{w}$ given by

$$
\left.\sin ^{2} \theta_{W}^{\prime \prime} \equiv \kappa, \sin ^{2} \theta_{W}\right]\left.s \equiv \sin ^{2} \theta_{W}\right|_{\text {univ }}+\left.\left(\Delta_{\kappa}\right)_{\text {nom- naiv }} \sin ^{2} \theta_{W}\right|_{s} .
$$

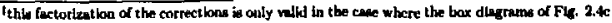
ine neglected. 


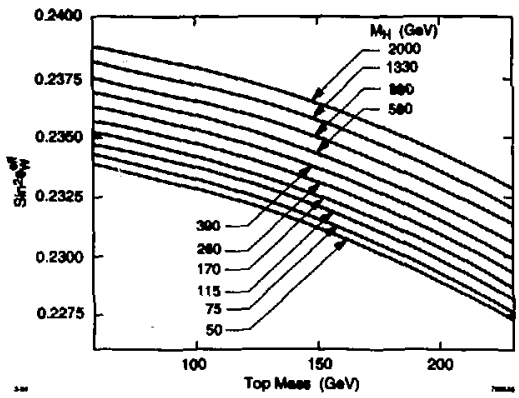

Figure 2.8: The dependeace of $\sin ^{2} \theta_{\psi}^{\prime \prime}$ wa $m_{\mathrm{t}}$ and $m_{\text {Higg }}$.

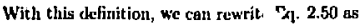

$$
-i \Lambda I_{Z}\left[4 \sqrt{2} G_{F} \rho_{J}\right]^{1} \gamma^{\mu}\left[\frac{1}{2}\left(1-\gamma^{s}\right) \gamma_{j}-\sin ^{2} \theta_{J} J^{\prime} Q_{J}\right] .
$$

Figure 2.8 shows the dependence of $\sin ^{2} \theta_{4}^{x / \prime}$ on the top und Higgs mosters, We note in passing thit. the "universal" wcrk mixing angle defined in Eq. 2.54 is the same as the $s_{*}^{2}$ of Kennedy aud Lynn[38], which is constructed to be independent of the icriex corrections.

Now, we ure sble to continue our discussion of eloctroweak physics ut the $Z^{\circ}$ pole, including the leading order corrections as we procued by replacing the couplings in the tree-level expreseions by their renormalized counterparts.

\footnotetext{
TTule dependerke wa caleuluted uning the program ZSHAPE(44), without the value of $\alpha^{-1}\left(M_{Z}^{2}\right)$ diven in Eq. 2.47.
} 


\subsection{Electrowalk Anymmetries: Testing the Standard Model}

As mentioned aloove, the measurentent of a different combination of the paranetern $g, g^{\prime}$, and $\left\langle\phi_{0}\right\rangle$ (or equivalently $\alpha, G_{F}$, and $M_{z}$ ) which specify the weak interaction can serve es a cest of the validity of the theory. Different coulsinntions of these parameters luve different sensitivity to the varions radiative corrections introduced by physical processery beyand the Standari Afodel. Thus, if a difference between prediction and experiment is seen, a compuriwen of the various observaljes may yield some intdication of its origin. Sluce the goul of these meagurcutents is precision in determining the tiectrowcak prameters, every attempt has becu mude to eliminate sources of experimental error. One powerful technique to accomplish this is to form ratios of cross sectious where the parameter of interest his been isolated by the angular- or polurization-dependence of the differential cross section. This has the aulvantage that the errors inherent in determining an sbsolute cross scetion, such ss those due to luminosity uormalization and absolute detuction efficiency, cancel in the asymuntry which is formed. Uking this met hod allows the isolation and examination of various combinations of the electroweak coupling. Sextral of these asymmetries will be discussed below.

Bofore we proceed, however, it, will be useful to rewrite the Born cross section for fermion production at the $Z^{0}$ prole $\left(\mathbf{E}_{\mathrm{f}} \mathrm{g} . \mathbf{2 . 4 1}\right)$ is follows:

$$
\begin{aligned}
\left.\frac{d a}{d \Omega}\right|_{z}= & K\left(v_{e}^{2}+a_{e}^{2}\right)\left(v_{j}^{2}+a_{f}^{2}\right) \times \\
& \left(j-A_{e} P_{e}\right)\left(1+\cos ^{2} \theta\right)+2 A_{J}\left(A_{e}-P_{k}\right) \cos \theta,
\end{aligned}
$$

with

$$
K=\frac{a^{2}}{4 \sin ^{3} 2 \theta_{u}} \frac{s}{\left(s-M_{z}\right)^{2}+\Gamma_{Z}^{2} s^{2} / M_{Z}^{2}}
$$

where we haw factored out the term $\left(v_{e}{ }^{2}+a_{f}{ }^{2}\right)\left(v_{f}{ }^{2}+a_{f}{ }^{2}\right)$ from the previous expreasion and have defined the rusantity

$$
A_{j}=\frac{2 v_{j} u_{f}}{v_{f}^{2}+a_{f}^{2}}
$$


for each species of fermion $f$. The difference of $A_{l}$ frow zero is a measure of the parity violation in the $Z f f$ coupling. The uigned longitudinal polarization of the electron bean will beverforth be referred to $P_{\mathrm{r}}$.

\subsubsection{The Left-Right Arymmetry}

Perhaps the simplest example of an ayymetry which can be formed in thin manner is the Left-Right Asymuetry, denoted $A_{L A}[45]$. At the SLC, the longitudinal polarizetoun of the clectron beam allows us to exanite the parity violation in $Z^{0}$ production directly by forming the ngymunetry

$$
A_{L R}=\frac{\sigma\left(\mathrm{e}_{R}^{+} E_{L}^{-} \rightarrow Z^{0} \rightarrow f f\right)-\sigma\left(e_{L}^{+} c_{R}^{-}-Z^{0} \rightarrow f j_{f}\right.}{\sigma\left(e_{R}^{+} e_{\bar{L}}^{-} \rightarrow Z^{0} \rightarrow f f\right)+\sigma\left(e_{L}^{+} e_{\bar{R}}^{-} \rightarrow Z^{0} \rightarrow f f\right)},
$$

where $f$ is any outgoing fermion. More specifically, from the expression from Eqe. 2.56 an $\div 2.40$ with $P_{e}=1$,

$$
A_{L . \mathrm{R}}=A_{\mathrm{r}}=\frac{2 U_{\mathrm{e}} \mathrm{a}_{k}}{v_{\mathrm{e}}^{2}+a_{\mathrm{e}}^{2}}=\frac{2\left[1-4 \sin ^{2} \theta_{W}^{t / J}\left(M_{Z}^{2}\right)\right]}{1+\left[1-4 \sin ^{2} \theta_{W}^{J J}\left(M_{Z}^{2}\right)\right]^{2}},
$$

Here, the dependence on the renormalized electruscak juranteters has boen introdured by replncing $\sin ^{2} \theta_{w}$ in Eq. 2.40 with $\sin ^{2} \theta_{w}^{* J}$. For arbitrary beam polarization with $P_{e}<1$,

$$
A_{L R}=\frac{1}{P_{e}} A_{m}=\frac{1}{P_{*}} \frac{N_{L}-N_{H}}{N_{L}+N_{R}} .
$$

where the quantity $A_{n n}$ is the measured usymutry, given by the difference between the number of $Z^{0}$ events produced with a loft-lianded clectron heam $\left(N_{L}\right)$ and the number produced with a righti-handed electron benm $\left(N_{R}\right)$ over the total. As can be secn from this exprcssion, the higher the beam polarization, the larger the asymmetry. For $P_{E} \sim 1_{2}$ the expected valiue of $A_{L R}$ is about 0.16 .

The quantity $A_{L R}$ la uniquely suited for a procision determination of the value of $\sin ^{2} \theta_{w \prime}^{f f}$ due to several inportant properties. Finst, can be fron Eq. 2.60, these is no dependence on the fingl atute fermion couplings. This inplies that all fimal atute fermions can be used to measure $A_{L R}$, which greatly incrences the statisticel

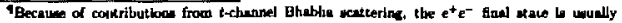
extuded.
} 
power of the messurement. This also inuplies that, if symmetric ncceptance limits wre chosen, $A_{L R}$ is invensitive to any final stute radiative orrections[46], such as thooe due to GCD. In addition, as long os the local detector eficiency for obverving fermions and antifermions is the same, $A_{\ell n}$ is independent of detector acceptance.

We give the sensitivity of $A_{L R}$ to $\sin ^{2} \theta_{L / I}^{\prime \prime}$ here as a benchmark againd which we can compare the other asymmetries:

$$
\frac{d A_{L R}}{d \sin ^{2} \frac{\theta_{W}}{W^{2}}}=-7.84
$$

which implies t'rat

$$
\delta \sin ^{2} \theta_{W^{\prime}}^{\prime \prime}=\delta A_{L R} / 7.84 .
$$

The sensitivities of all of the electromeak asymunct ries ure given in Tabte 2.4 .

The SLD Collaboration has perfonted two measurewents of $A_{\text {LR }}$ based on ruuning during 1992 and 1993 . The combinerl results for $A_{L A}$ and for din $^{2} \theta_{\mathrm{K}}$ If $^{\prime}$ after radiative corrections have been applied are[4]7]:

$$
\begin{aligned}
A_{L R}^{0} & =0.1637 \pm 0.0075 \\
\sin ^{2} \theta_{W} W^{\prime} & =0.2204 \pm 0.0010 .
\end{aligned}
$$

\subsubsection{The $\tau$ Polarization Asymmotry}

The angular cortelatione from the teenys of the fund state fermions can also be analyzed to provide information on the electroweak couplings. This measurement is most easily made in $T$ decays, ne it is the paly ferniun which can be "spin-analyzed" in a convenient manner, i.e., its charge is readily identified and it often decays two a two-body final state whose helicity components can be measured. Since the natural polarization of the final state fermion is used in this analysis, longitudinal beam polarization is not necessary. We can form the final state polarization asymatetry between the production of kft-lanuled $\left(\tau_{L}\right)$ and right-handed $\left(\tau_{R}\right)$ taus:

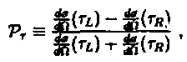


where the $r$ helicity is obtained by examining its decay. If we insert the unpolarized fermion production rroes section, we find

$$
\mathcal{P}_{\tau}=\frac{2 A_{r} \cos \theta+A_{f}\left(1+\cos ^{2} \theta\right)}{\left(1+\cos ^{2} \theta\right)+2 A_{\mathrm{r}} A_{f} \cos \theta} .
$$

This asymetry bas two intercsting pruperties. First, if it is integrated over a $\cos \theta$ symmetric acceptance, the dependence on the initial state coupling drops out, lesving

$$
\left\langle\mathcal{P}_{\mathbf{t}}\right\rangle=A_{\boldsymbol{r}} .
$$

Since the electroweak quantum numbers of the $\tau$ and the electron are julentical, this quintity has the ane sensitivity to $\sin ^{2} \gamma_{W}^{f \prime}$ as $A_{L R}$, namely

$$
\frac{d\left(\mathcal{P}_{r}\right)}{d \sin ^{2} \theta_{W}}=-7.84 \text {. }
$$

Second, by measuring the forwurd-backward asymmetry in $\mathcal{P}_{\boldsymbol{t}}$, one cout isolate $A_{e}$, which provides yet another way to accoss $\sin ^{2} \sigma_{\psi}^{e f J}$. Thus, the $\tau$ polarization asymmetry is a remarkable tool with which to probe the clectrowenk sector. Unfortunately, the fraction of $Z^{0}$ decays which prejuce a $\tau^{+} \tau^{-}$pair is only $3 \%$, and less than half of these exnits contain a $\tau$ decay mode suituble for spin unalysis. In addition, the anklyzing power of the helicity aralysis is not unity, which further dilutes the measured asymmetry. All told, each $Z^{0}$ event has appraximately 200 times less statistical wejglt for the $\tau$ polarizution asymmetry than is dues for $A_{L F}$. Comparisons of the statistical weight for each of the asymmetries are also summarized in Table 2.4 .

\subsubsection{The Forwerd-Bacloward Asymmetry}

The fermion forwyrd-backward asymmetry, which onc can measure at any $e^{+} e^{-}$experintent, provides information on the initial and finsl state couplings of the rectroweak interaction. This method has been usod at lower centur-of-mnss energies[48] to measure the extent of $\gamma-Z$ interference. At the $Z^{0}$ pole, it allows direet access to the fermion- $Z$ couplings throigh the parity-violation prewent in ayy weak interaction. We define the forward-backward asymmetry for a fermion of type $f$ as

$$
A_{F B}^{f} \equiv \frac{\sigma^{f}(\cos \theta>0)-\sigma^{S}(\cos \theta<0)}{\sigma^{J}(\cos \theta>0)-\sigma^{f}(\cos \theta<0)}=\frac{3}{4} \frac{2 v_{\varepsilon} a_{c}}{4 v_{c}^{2}+a_{c}^{2}} \frac{2 v_{f} a_{f}}{v_{f}^{2}+a_{f}^{2}}=\frac{3}{4} A_{c} A_{f} .
$$


Tuble 2.4: A comparison of the different observable fermicis production usymetries that $\cdots$ ' we messured at the $Z^{n}$ pole. Tho relative statistical sensitivities of each measurement (last coltumn) are calculated hy asuming reasongble sclection efficiencies for cach of the thalyses. Those meusurements using h quarks are ussumed to have been performed with a lepton tuy. The inagnitude of the longitidinal beam polarization is assumed to be $63 \%$. The size of the messured asy mineter isus not been taken into eccount.

\begin{tabular}{|c|c|c|c|c|}
\hline Parameter & Couplings & Raw Asymemetry & $d A / d \sin ^{2} \theta_{W}$ & Fel. Stat. Sens. \\
\hline$A_{L A}$ & $A_{e}$ & $\left|P_{v}\right| A_{e} \sim 0.1$ & -7.84 & 1 \\
\hline $\mathcal{P}_{\tau}$ & $A_{T}$ & $A_{\tau} \sim 0.16$ & -7.84 & 0.0075 \\
\hline \multirow[t]{3}{*}{$A_{F D}^{J}$} & $A_{e} A_{b}$ & $\frac{3}{4} A_{c} A_{b} \sim 0.11$ & -5.59 & 0.015 \\
\hline & $A_{\mathrm{e}} A_{\mathrm{c}}$ & $\frac{3}{3} A_{c} A_{\mathrm{c}} \sim 0.08$ & -4.39 & 0.001 \\
\hline & $A_{\varepsilon} A_{t}$ & $3-t_{\ell} \sim 0.02$ & -1.92 & 0.017 \\
\hline \multirow[t]{3}{*}{$\dot{A}_{F B}^{\prime}$} & $A_{b}$ & ${ }_{3}^{3} \mid P, A_{b} \sim 0.44$ & -0.63 & 0.0018 \\
\hline & $A_{e}$ & $\frac{3}{4}\left[P, A_{e} \sim 0.32\right.$ & -3.42 & 0.0008 \\
\hline & $A_{t}$ & $\frac{3}{4}\left[P_{1} A_{\ell} \sim 0.0 \mathrm{~s}\right.$ & -7.84 & 0.00 \\
\hline
\end{tabular}

If we take into uccount the symm :ric acceptunce of a real partiele detector which extends to some $\cos \theta_{0}$, this expres: on uexuires a $\cos \theta$ dependence:

$$
A_{F D}^{\prime}=\frac{\int_{0}^{\infty} \operatorname{\theta os} \theta_{0} d \cos \theta \frac{d \sigma_{f}}{d \Omega}-\int_{-\cos \theta_{11}}^{0} d \cos \theta \frac{d \sigma_{j}}{d \Omega}}{\int_{-\cos \theta_{0}}^{\cos \theta_{0}} d \cos \theta \frac{d \sigma_{\rho}}{d \Omega}},
$$

which yields, upon integration,

$$
A_{\mathrm{Fb}}^{f}=\frac{3}{4} \frac{A \cos \theta_{0}}{3+\cos \theta_{0}^{2}} A_{\mathrm{r}} A_{\mathrm{f}} .
$$

Since in the case of $A_{F B}^{f}$ ihe pro :ct $A_{C} A$, is measured, one cannot disentangle the Initial and final state couplings. $T l_{\text {. }}$. ineriments at LEP have used these asymmetries as a method to derive information $1 n \sin ^{2} \theta_{W}^{\prime f}$ by using the full $\sin ^{2} \theta_{W /}^{\prime / d e p e n d e n c e}$ of both the initial and final state c uplings. As will be scen below, the quantity $A_{I}$ 
is large for quark final states, which implics that the statigtical sensitivity of this method to $\sin ^{2} \theta_{t \prime \prime}^{\prime \prime}$ can be large if one can efficiently melect samples of quarks whose charge and production angle can be measured. In terms of raw sensitivity to $\sin ^{2} \theta_{y^{\prime}}^{\prime \prime}$ we have

$$
\begin{aligned}
& \frac{d A_{F H}}{d \sin ^{2} \theta_{W}}=-5.58 \\
& \frac{d A_{F H}^{\mu}}{d \sin ^{2} \theta_{H}}=-4.39 \\
& \frac{d A_{F H}^{\prime}}{d \sin ^{2} \theta_{W}}=-1.92
\end{aligned}
$$

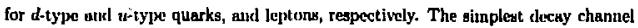
in which to nacasure $A_{F B}^{\prime}$ is $Z \rightarrow e l i$. The small branching fraction of the $Z^{0}$ to leptons, conjlexl with the sutell sonsitivity to $\sin ^{2} \theta_{W}^{\prime \prime}$ and the small summetry size $(\sim 2 \%)$ linit the statistical sensitivity of this method. The asymmetry in the case of $b$ quarks is lagge $(\sim 11 \%)$, and it also has $n$ large sersitivity to $\sin ^{2} \theta_{W}^{\prime J}$; the use of this channel is only limiled by the ability to correctly deternine the production direction of the $b$ (ws opposed to $\bar{b}$ ) quark. Teduniques for acconplishing this deternination will be discussed in Sextion 2.8 , below.

By alternuting the longitudingl polarization of the electron beam, we can form a double nsymmetry, the so-called left-righ for what beckward asymmel ry $\hat{\lambda}_{F B}^{l}$ (49):

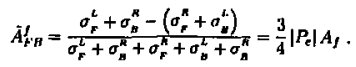

- In the above expression, $\sigma_{p}^{2}$ denotrs the cross section for producing the fermion $f$ in the forward hemisphere $(\cos \theta>0)$ with a keft-hended electron bean. This shows the experimental advuntage in using a longitudinally polarized beam: by forming the double asymmetry, the $Z^{0}$ production esymmetry caused by the parity violation in the initial statc coupling has been removed, leaving experimentally accesvible the find state coupling. In addition, for substantial net berum polarization, the raw asymmetry $A_{r B}^{\prime}$ is quice large compared to the stenclurd forward-bodkward abymmetry $A_{F o}^{\prime}$, since the mulciplicntive factor $P_{t}$ can, in principle, be $\sim 1$, whereas $A_{\varepsilon} \sim 0.16$. This makes 
for a smaller fractional error on the measurentetit of $A_{f_{u}}^{f}$ conituared with that for $A_{F B}^{f}$ Ior a similar data set.

For completenes, wo give here the angular dependence of the naymmetry as de. rived frotn Eq. 2.56:

$$
\dot{A}_{F D}^{\prime}=\left\{P_{t} \mid A_{f} \frac{2 \cos \theta}{1+\cos ^{2} \theta} .\right.
$$

This thesis presents the first memarement of $A_{c}$ using exclusively reconstructed cliurm mesons.

We turn now to a discusolon of the rediative corrections that effect the messurewent of $\dot{A}_{F B}$.

\subsection{Radlative Corrections to $\tilde{A}_{F U}$}

We divide the elescription of the effects of indiative corrections on $\bar{A}_{F \|}$ into two sections: the electromeak corrections, which are sunesl, mol the QCD corrections, which can be large and naust be considered in detail.

\subsubsection{Electroweak Corrections}

The prinary contribution to the ehetromenk corrcetions of $\vec{i}_{F B}$ (Hercafter denoted

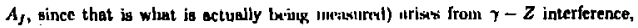

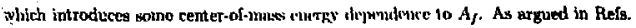
[wi] and [35], contributions from the wivk interuttion wertex corrections and the box

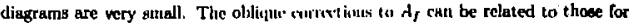
$A_{\text {LH }}=A_{e}$ by

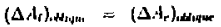

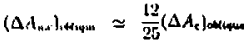

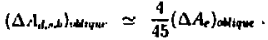

Thoe corrections due to QEU initial stute rudiation have the effect of eltifting the effective center-of-muss cnergy. To desuonstrate the size of these cfiecty, we show in 


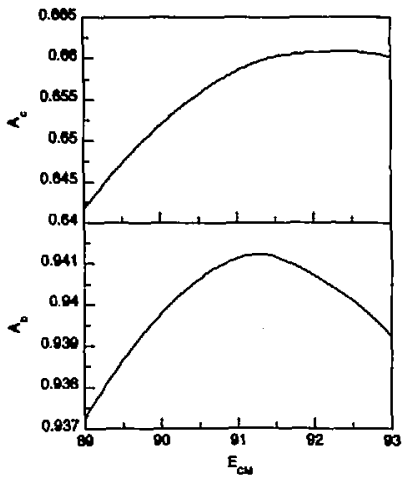

Figure 2.9: Electroweak-corrected valucs of $A_{b}$ and $A_{c}$ vs. $\sqrt{s}$ near the $Z^{0}$ pole. QCD radiative corrections are not included. The tree-level values are $A_{c}=0.67$ and $A_{b}=0.93$ for comparison. These values have been calculated using the ZFITTER package[5I].

Figure 2.9 the wilues of $A_{\varepsilon}$ and $A_{4}$ us. $\sqrt{5}$ near the $Z^{\circ}$ pole with the electrowenk radiative corrections appliedThese values hase been calculated using the ZFITTER pexkage[51].. One can see that the variation of these anymmetry parameters with respect to the center-of-masa chergy, as well as the magitude of the corrections, is small, as expected. 


\subsubsection{QCD Corrections}

\section{$O\left(a_{\text {s }}\right)$ Correction}

First order QCD cortections to the beavy quark for ward backward asymmetry heve been calculated by a number of groupa| $[52,53]$. The full differential croses section for massive quarks including $\mathcal{O}\left(a_{*}\right)$ corrections is given by[jo2]

$$
\begin{aligned}
& \frac{d^{2} \sigma_{t}}{d \Omega}=\frac{3}{4}\left(\frac{\alpha}{4 \sin ^{2} 2 \theta_{W}}\right)^{2} \frac{\beta}{\Gamma^{2}} \\
& \times\left\{( v ^ { 2 } + a ^ { 2 } - 2 a v P _ { - } ) \cdot \left[\left(v_{f}^{2}+a_{f}^{2}\right)\left(1+\frac{h_{f}^{2}}{2}\right)\left(1+\frac{4 \alpha_{f}}{3 \pi} F_{1}\left(\bar{n}_{I}\right)\right)\right.\right. \\
& \left.-\frac{3}{2} a_{f}^{2} \bar{m}_{f}^{2}\left(1+\frac{4 \alpha_{2}}{3 \pi} F_{4}\left(\bar{m}_{f}\right)\right)\right]\left(1+\cos ^{2} \theta\right) \\
& +\left(v^{2}+a^{2}-2 u v P_{-}\right) \cdot\left[\left(v_{l}^{2}+a_{j}^{2}\right)\left(\frac{\bar{m}_{l}^{2}}{2}+\frac{4 a_{t}}{3 \pi} F_{2}\left(\bar{m}_{j}\right)\right)\right. \\
& \left.-a_{j}^{2} \frac{\tilde{m}_{f}^{2}}{2}\left(1+\frac{4 \alpha_{f}}{3 \pi} F_{5}\left(\tilde{m}_{f}\right)\right)\right]\left(1-3 \cos ^{2} \theta\right) \\
& \left.-4\left(2 v a-\left(v^{2}+a^{2}\right) P_{-}\right) a_{j} v_{f} \beta\left(1+\frac{4 a_{j}}{3 \pi} F_{3}\left(\hbar_{j}\right)\right) \cos \theta\right\} .
\end{aligned}
$$

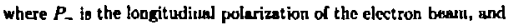

$$
\begin{aligned}
& \frac{4 \alpha_{i}}{3 \pi} F_{1}\left(\bar{m}_{f}\right)=\frac{\alpha_{d}}{\pi}\left(1+3 \bar{m}_{f}^{2}\right) \\
& \frac{4 \alpha_{1}}{3 \pi} F_{2}\left(\bar{m}_{l}\right)=\frac{\alpha_{1}}{\pi}\left[\frac{2}{3}-\frac{x^{2}}{6} \bar{m}_{f}-\bar{m}_{f}^{2}\left(\frac{1}{3}+\ln \frac{m_{l}}{2}+\frac{1}{3} \ln ^{2} \frac{\bar{m}_{l}}{2}\right)\right] . \\
& \frac{4 a_{t}}{3 \pi} F_{3}\left(\bar{m}_{f}\right)=\frac{a_{s}}{\pi}\left[\frac{8}{3} n_{l}+m_{f}^{2}\left(\frac{7}{3}+\frac{\pi^{2}}{18}-\frac{2}{3} \ln \frac{\bar{m}_{f}}{2}+\frac{1}{3} \ln ^{2} \frac{\bar{m}_{f}}{2}\right)\right], \\
& \frac{4 a_{4}}{3 \pi} F_{4}\left(\bar{m}_{f}\right)=\frac{a_{0}}{\pi}\left(3+4 \ln \frac{m_{f}}{2}\right) \text {. } \\
& \frac{4 a_{1}}{3 \pi} F_{0}\left(\bar{m}_{f}\right)=\frac{a_{s}}{\pi}\left(5+4 \ln \frac{m_{f}}{2}\right) \text {. }
\end{aligned}
$$

The quantity $\bar{m}$ is the scaled heavy quark mass, with velocity $\beta^{2}=1-\bar{m}_{f}^{2}$. The expresaions in Equation 2.74 are correct to order $m_{f}^{2}$. 
Forming the asymmotry $\dot{A}_{F B}$ revults in

$$
\begin{aligned}
\dot{A}_{F n}(0)= & \frac{3}{4} \beta \frac{2 v a}{v^{2}+a^{2}} 2 v_{j} a_{f}\left(1+\frac{4 a_{f}}{3 \pi} F_{3}\left(m_{f}\right)\right) \\
& \times\left\{\left(v_{f}^{2}+a_{f}^{2}\right)\left(1+\frac{\bar{m}_{l}^{2}}{2}\right)\left(1+\frac{4 a_{I}}{3 \pi} F_{1}\left(\bar{m}_{f}\right)\right)\right. \\
& \left.-\frac{3 \bar{m}_{f}^{2}}{2} a_{f}^{2}\left(1+\frac{4 a_{s}}{3 \pi} F_{4}\left(m_{f}\right)\right)\right\}^{-1} .
\end{aligned}
$$

We write it in the form

$$
\ddot{A}_{F R}(0)=\dot{A}_{F B}^{0}(0)(1-\Delta),
$$

with the exact lowest order anymetry

$$
A_{F B}^{0}(0)=\frac{3}{4} \beta \frac{2 a v}{v^{2}+a^{2}} \times \frac{2 a_{f} v_{f}}{v_{j}^{2} \frac{3-\beta^{2}}{2}+\beta^{2} a_{f}^{2}},
$$

and the radiative correction to first order in $\alpha$, and second order in $\bar{m}_{f}$, given by

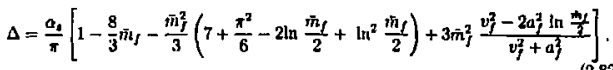

to $\mathcal{O}\left(a_{a}\right)$ and $\bar{m}_{f}^{2}$. Note that the correction has the expected sign: glwon rodiation must dilute the asymmetry, as it tends to stnear out the initjal quark directions, in most cases smearing them away fron the region at high $\cos \theta$ where $A_{F B}$ is large. This is easily seen in the limit where the radiated gluon is very energetic. In this ease, the observed qurk directions are essentially unrelated to their directions before gloon radiation, wid the quarks can end up anywhere in the detector.

These analytic formulac have been incorporated numerically into the differential craes section that is used in our measurement of $A_{c}$. In Figure 2.10 we ahow the effects of the $O\left(a_{4}\right)$ corrections for massless and maseive quarks.

\section{$\mathcal{O}\left(n^{2}\right)$ Corrections}

Second order calculations for the QCD radjative corrections (with mussive quarks) to A) lsave been perforined[54]. The total correctione have the form

$$
A_{c}^{\text {mesus }}\left(\mathcal{O}\left(\alpha_{a}^{2}\right)\right)=A_{c}^{0}\left(1+\left(\frac{\alpha_{n}}{\pi}\right)^{2}\left[-(4.4 \pm 0.4)-(26 \pm 6) \int D_{s}\right]\right)
$$




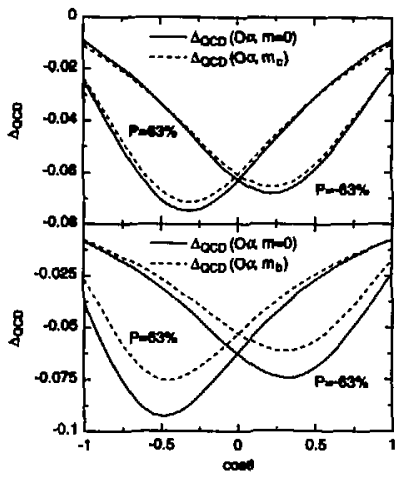

Figure 2.10: The $\mathcal{O}\left(\alpha_{2}\right)$ QCD corrections to $A_{b}$ and $A_{c}$ for maseless (solid line) and maswive quarks (dotted lise). The quantity $\Delta_{Q C D}$ relates the uncorrecled asymetry $A^{0}$ to the measured asymuetry by $A^{\text {meas }}=A^{0}\left(1+A_{\alpha C b}\right)$.

for c quarks, and

$$
\left.A_{*}^{\text {meas }}\left(O\left(0_{*}^{2}\right)\right)=A_{4}^{0}\left(1+\left(\frac{u_{4}}{\pi}\right)^{2} !-(1.9 \pm 0.4)-(3.5 \pm 0.7) f_{D_{*}}\right)\right) \text {. }
$$

for $b$ quarks. These corrections are jist the next order contributions: they niust be applled in addition to the leading order calculation shown above. The secoud order correction depends in detail on the seceptance of analysty cuts for four-jet ovents whereiu a radjated glion emerges as a puir of henvy quarks. In principte, in the cabe where the two primary quarks are one of the light flavora, these events ahould be treated a QCD corrections to the light quurk production cross section. Since the 

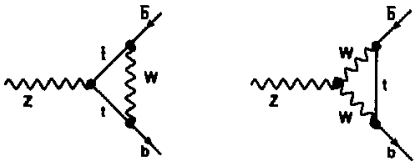

Figure 2.11: Radiative Corrections to $A_{4}$ from virtual top quark loop .

beavy quarks can cause the event to be tasged as part of the heavy flavor surnple, however, they need to be coneldered, as they will nodily the measured anymutritry by a factor proportional to their probability to pass the tvent selection criterin. This experimental acceptance is paramatrized by the quantity $f_{V_{B}}$ and must be determined by Monte Carlo simulation. Tise errors quoted are due to the ambiguity of what value the quark mases should take in the calculation, whote the ranges asoumesl are $4 \mathrm{GeV} / c^{2}<m_{b}<5 \mathrm{GeV} / c^{2}$ and $1.1 \mathrm{GeV} / c^{2}<m_{c}<1.7 \mathrm{GeV} / c^{2}$.

\subsection{Pósible Modifications to $A_{f}$ from New Physics}

The values of the fermion asymmetries can be modified by any new physics that can change the final state through corrections to the $Z J f$ vertex. The value of $A$, alrcady recrives a correction of this type from the presence of diagrams contuining virtual top quarks, as shown in Figure 2.11, whose effects are proportional to $m_{i}^{2} / M_{W}^{2}\left|V_{W}\right|^{2}$. The

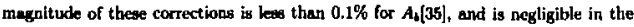
case of $A_{e}$, nince the corresponding diagrams contain strange quarks instead of top quarke. It is through diagrams sisch as these, however, that the influence of other, heavy perticlea can enterl.

The semitivity of the parameter $A_{f}$ to modifications of the electroweak coupling

\footnotetext{
ITwe conelder axtions for thin mation are drawn from Raforwace (35).
} 
caul be parametrlzed in torms of the difference of the observed $A_{f}$ and the standard unosel prediction $A_{f}^{\mathrm{yAr}}$. If the sarinble $\eta$ is introduced, where

$$
\frac{A_{f}}{A_{j}^{\text {gh }}}=1+\eta
$$

variations in the asymmetry parameters can be expressed in terms of changes in the vector $\left(v_{f}\right)$ and axial vector $(a f)$ couplings of the fermions. A short calculation yields

$$
\begin{aligned}
\eta_{J} & =\frac{2\left(1-c_{j}^{j}\right)}{c_{f}\left(1+c_{f}^{2}\right)}\left[\delta v_{j}-c_{j} \delta n_{J}\right] \\
& =\frac{2\left(1-c_{J}\right)}{c_{f}} \frac{\left(1+c_{f}\right)^{2}}{1+c_{f}^{2}}\left[\delta g_{H}^{J}+\left(\frac{1-c}{1+c}\right) \delta g_{L}^{\prime}\right],
\end{aligned}
$$

where $c_{f} \equiv v_{f} / a_{J}=1-8 / 3 \sin ^{2} \theta_{w}$, and the couplings have been rexpresed as $v_{f}=g_{L}^{f}+g_{h}^{f}$ und $a_{f}=g_{L}^{f}-g_{k}^{f}$ so as to give expressions independent of the value for $\sin ^{2} \theta_{w}^{c f l}$. Assuning $\sin ^{2} \theta_{w}=0.2 \mathrm{~J} 25$, we obtxin

$$
\begin{aligned}
& \eta_{c} \simeq 5.43\left[\delta_{k}+0.45 \delta g_{i}^{c}\right] \\
& \left.\eta_{k} \simeq-1.74\left[\delta_{t}^{*}+0.18 \delta \delta_{t}\right]^{\prime}\right]
\end{aligned}
$$

This shows that, in fact, $A_{e}$ is more sensitive in deviations of the couplings if the changes were to occur equally for $b$ and $c$. Since charm quarks have the light strange quark in their weak isoxpin doublet, however, most effects tlitt arive from new phystcs in the vertex couplings are murkedly smaller for charm quarks when compared to those for $b^{\prime} \mathrm{s}$. In the following sactious, we briefly review how soano of the potential extenwions of the standard model coukd wodify the values of the heavy quark asymmetries $A_{f}$, with particular attention to thown: proceses which could result in large modifications of the $Z c c$ coupling.

\subsubsection{Two-Higgs Doublet Models and Supersymmetry}

When the single higgs doublet of the minitnal standard model as described atrove is extended to two donblets, the single "standerd nockel" higge perticle is replaced by five higgs bosons, each of which can participace in vertex loopo and hence, cen effect 
the heavy quark asymmetries[50]. Typically, three neutral higes $h^{0}, h^{0}$, and $A^{0}$, and two charged biges $H^{ \pm}$, comprise the higes spectrum of these theories. Theories of this type are parametrized by the ratio of the vacuum expectation values of the two doublets $t_{2} / v_{l} \equiv \tan \beta$. Charged higes and (in aupersymmetry) chergino exchange in $Z \rightarrow b \bar{b}$ decayn can have potentialy lerge effects on the effectjvo $Z b \bar{b}$ coupling $[57]_{i}$ these modlfications are suppresed by $\left|V_{\infty}\right|^{2}<2 \times 10^{-3}$ for charm. Corrections from neutral higes exchange cast be proportional to $m_{f}$, and thus are amall $a$ well. Other contributions from ueutralino exchange and squark contribution in supersymmetric theory are also expected to be small for charm.

Alternate snodels with two higgs doublets can contain flavor-changing neutral currents[58], where the effects are largest in the $t-c-$ higgs vertex. This is sdvantageous, as it allows a direct coupling of $c$ to the "beavy" physics npened up by $m_{t}$. Depending on the strength of the Yulawa coupling in these theoties, large (> $5 \%$ in $\delta A_{\mathrm{c}} / A_{<}$) could be oberived.

\subsubsection{Extended Gauge Sectors}

Or the various models for extending the gange structure in which the Stundard Model is emlxwkled in order to aclieve a unification of couplings, the $E(6)$ models[59] are interesting to our present discusion because they call contain "Alternute Left-Right" models that effectively switch the quantuni numbers of the ordinury fermions[60]. This leads to large vertex corrections for charn quark final states which are similar to those seen for $b$ quarks in the Stundard Mlodel, which could potentinlly add large corrections to the $Z c c$ vertex. The ratio of the higes mans to the mas of the $W_{R}$ boon that mediates the interaction is the determining fictor in the ajze of the modification of $A_{c}$ duc to a model of this type. For $m_{W_{n}}>0.3 \mathrm{TeV}$ and higgs mases greater than $\sim 1 \mathrm{TcV}, 10 \%$ deviations from the expected Stunderd Model value is possible. 


\subsection{Measurements of the Heavy Quark Forward- Backward Asymmetries}

Ax stomn in Table 2.4, the quakto produced in $Z^{\circ}$ decays have large anymetry parumeters $A_{f}$, which injlies that the prodect $\frac{3}{4} A_{e} A_{f}$ is aleo relutively large, or at

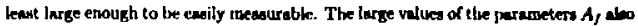
imply that the sensitivity of $A_{F B}$ to variations in the value of $A_{\text {r }}$ is nearly maxime (bil Table 2.4), which motivates the effort to tnesure exch separately in order to search for differoness in the initial and lind sate couplings. The atatistical boon provided by the large hadrunic width of the $Z^{0}$ provides extra incentive to attempt measurements of $A_{F D}$ for quertes.

In order to be sufficiently sengitive to the guark forward-backward asymmetries, however, us pure sample as paseible of the particular quark flavor is needed. With the cechniques presently swilable in modern perticle detectors, the ooly imo prinary quarks whoee events can be isolated in a "clean" (rcad, "with sinall systematic errors") manner are $c$ and $b$. These tronsiderations have led to many measurerdents of Afs for heavy quarks by the LEP experinuents in order to guin as much information $=$ possible about the value of $\sin ^{2} \theta^{\prime \prime \prime}$. We now turn to a discussion of the various techniques used to obtain samples of theary quarks for asymmetry mesouremenks. Ao will be secn, all of these techniquess exploit one or more of the feat ures of heavy quark production and decay:

- Hard Fragmentation Functions",

- Large Mnsses,

- Long Lifetimes.

Results of the varions methods will be discusent in Chapter 1.

\footnotetext{
-See Chaper 3 .
} 


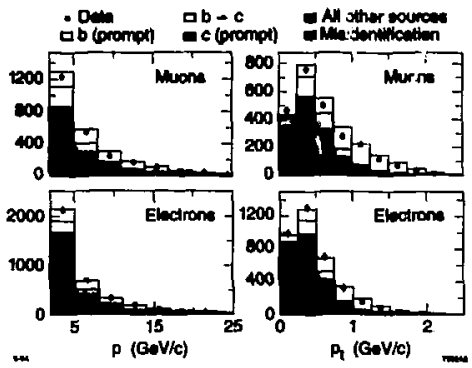

Figure 2.12: The $p$ and $p_{1}$ distributions for keptone in hadronic $Z^{0}$ decays.

\subsubsection{High $p_{\perp}$ Lepton Thgs}

The first inclusive method used to isolate leavy quark events[61] was to require the presence of high-ewergy, isolated leptors as a tag. This technique exploits the relatively large $(\sim 20 \%)$ benching fraction of heavy hadrons to kptons pliss a hadronic system $(Q \rightarrow \ell+X)$ the hard heavy quark fragmentation functions, and the large energy avalable in the heavy hadron decay. The latter two properties of henvy quark eventa result in leptons with large total momentum and large $p_{2}$ relative to the injtial heavy hatron directlon, which is commonly approximated by the jet axis' or the event thruat[62] axis.

The possible nources of keptons in hadronic decays of the $Z^{0}$ aret:

$\bullet b \rightarrow e^{-}$

$-c \rightarrow e^{*}$

\footnotetext{
'Ses Chepter 8.
}

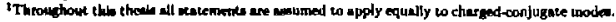


- $b \rightarrow \mathrm{c} \rightarrow \ell^{+}$

- $b \rightarrow \bar{c} \rightarrow t^{-}$, where the $\bar{c}$ is produced from the bresching of the $W^{-}$

$\bullet b \rightarrow \tau^{-} \rightarrow \ell^{-}$

- punchthrough, cleceys of $\pi s$ or $K s$ in filght

and the decays that proceed through uixing.

- $b \rightarrow \bar{b} \rightarrow \ell^{+}$

- $b \rightarrow \bar{u} \rightarrow c \rightarrow \ell^{-}$, etc

The sign of the outgoing lepton is noted here expluctly, as it is used to determine the sign of the purent heavy quark in onder to determinc whether the fermion wa produced in the forwurd or bsckward hemispluere. From the list above, it is clear that there is the posibility of confusion, as a kepton can come from any of the wources. As shown in Figure 2.12. however, those leptons with high momentum and higb transverse mosuentun relative to the jet axis actually do come predominantly from heavy quark deckys. For $b$ events in SLD, the purity of the remaiuing semplo after a cut which excludes leptons that fall within the ellipse

$$
\left(\frac{p}{16}\right)^{2}+\left(\frac{p_{\perp}}{1.2}\right)^{2}=1
$$

is approximately $72 \%$ [166]. The efficieucy for $b$ 's in the hadronic event sample to pass this cut is about $5 \%$. As the momentun requirements are lowered, a significant portion of the leptors from charm dechys are allowed into the sampte. As long as the proper corrections for $B-\bar{B}^{0}$ mixing at includel, one can perform a muxitnum likelihood fit to the lepton spectra vs. polar angle and determine $A_{F \Delta}$ or $A_{F}$ and $A_{F H}^{c}$ Binultaneously. Tuls method has been employed by the LEP experimenta|63) to measure $A_{F B}^{+}$and $A_{F B}^{c}$. It should be mentioned that the erros on $A_{F B}^{e}$ derived from an aralysis of this type tend to be significantly larger than those on $A_{F B}$ due to the effective background suberaction that occurs when fitting the asymmetry of a charm sample with small vigad-to-noise. 


\subsubsection{Exclusive Reconstruction of Charmed Mesons}

It is pussible to obtein a relatively pure sample of events containing primary charm quark, but at the prec of requirios the presence of specific fintul states in the chermt meson decay. Since methode of this type will be the peimary focus of this theath, we will discus them in some detail here. These analyses usually consist of kinematically reconstructing topologically imple decay modes of the $D^{0}$ meson, then requiring that the $D^{0}$ was produced in the decay casade $D^{*+} \rightarrow \pi_{0}^{+} D^{0}$. This second requirement reduces the tagsing cfficiency, but enthances the tag purity through a clever kineinatic trick[64]. Since the masses of the $D^{++}$and $D^{0}$ are $2010 \mathrm{MeV} / \mathrm{c}^{2}$ and $1864 \mathrm{MeV} / \mathrm{c}^{2}$, respectively, the mas difference between the $D^{+}$and the $\pi_{0}^{+} D^{0}$ combination is os gentially a $\delta$-function at zerol. The distribution is guneared out slightly due to the extrenkly small $\pi_{0}^{+}$momentum $(39 \mathrm{MeV} / c)^{\top}$ in the $D^{n+}$ rest frame and from tracking resolution cffects. The kinematics of this decay result in a srong correlation between the angle of the $\pi_{*}^{+}$with respect to the $D^{0}$ direction and the energy of the $\pi_{*}^{+}$. Since tracking syntems tend to measure angins better than monenta, the width of the peak in the mas difference $(\Delta m)$ diatribution is much narrower than that for the $D^{4}$ mas. Significantly more background is excluded by cutting on the reconatructed $D^{0}$ mas and the $\Delta m$ distribution simultaneously. The added advantage in using this technique in a forward-barkward abymmetry meaurement is that the sign of the $\pi_{*}$ gives the sign of the $D^{\text {*+ }}$ aud hence the aign of the charm quark without any of the anbiguitles anociated with leptons.

In addition, one can take adventage of the hand charm fressuentation function to obiain a sample of even higher purity, as the buckground overies and Da produced in $b$ decny cascades typically lave baver energy. Requiring the frectionel energy $x_{b}=2 E_{D} / E_{C \mu}$ of the chanu systern to be more than 0.4 , for example, reduces the backgroulud contamination by another large factor.

To extimate the usefulnes of this tasging techuique, lel us first calculate the fraction of $Z^{0} \rightarrow c \bar{c}$ events which tontuin the decay chain $D^{*+} \rightarrow \pi_{ \pm}^{+} D^{0}$. . This lest

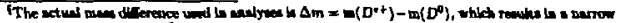

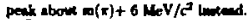

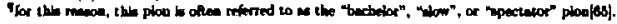


Table 2.5: The largest branching fraction in $D^{0}$ decay. We abow bere the branching [ractions into topological flnal stateo ignoring any reaconent substructure.

\begin{tabular}{|c|c|}
\hline Decay Mode & Branching Frection (\%) \\
\hline \multicolumn{2}{|l|}{ Seuni-teptanic Modes: } \\
\hline$D^{0} \rightarrow K^{-} e^{+} \nu_{e}$ & $3.80 \pm 0.22$ \\
\hline$D^{0} \rightarrow K^{-} \mu^{+} \nu_{\mu}$ & $3.2 \pm 0.4$ \\
\hline$D^{0} \rightarrow \boldsymbol{H}^{-0}-\pi^{0} e^{+} \nu_{r}$ & $1.6_{-0.5}^{+1.3}$ \\
\hline$D^{0} \rightarrow \bar{K}^{0} \pi^{-} e^{+} \nu_{r}$ & $2.8_{-0.6}^{+1.7}$ \\
\hline \multicolumn{2}{|l|}{ Hedronic Modes: } \\
\hline$D^{0} \rightarrow K^{-} \pi^{+}$ & $4.01 \pm 0.14$ \\
\hline$D^{0} \rightarrow K^{0} \pi^{0}$ & $2.05 \pm 0.26$ \\
\hline $\begin{array}{l}D^{0} \rightarrow K^{0} \pi^{+} \pi^{-} \\
D^{0} \rightarrow K^{-} \pi^{+} \pi^{0}\end{array}$ & $\begin{array}{c}5.3 \pm 0.6 \\
13.8 \pm 1.0\end{array}$ \\
\hline $\begin{array}{l}D^{0} \rightarrow K^{0} x^{0} x^{0} \\
D^{0} \rightarrow K^{-} \pi^{+} \pi^{+} \pi^{-}\end{array}$ & $\begin{array}{c}\sim 1.0 \pm 0.2 \\
8.1 \pm 0.5\end{array}$ \\
\hline$D^{0} \rightarrow K^{0} x^{+} \pi^{-} \pi^{0}$ & $9.8 \pm 1.4$ \\
\hline$D^{0} \rightarrow K^{-} \pi^{+} \pi^{0} \pi^{0}$ & $15 \pm 5$ \\
\hline$D^{0} \rightarrow K^{-} \pi^{+} \pi^{+} \pi^{-} x^{0}$ & $4.3 \pm 0.4$ \\
\hline$D^{0} \rightarrow K^{0} \pi^{+} \pi^{-} \pi^{0} x^{0}\left(\pi^{0}\right)$ & $10.6_{-30}^{+7.3}$ \\
\hline$D^{0} \rightarrow \pi^{-} \pi^{+} \pi^{0}$ & $1.6 \pm 1.1$ \\
\hline$D^{0} \rightarrow \pi^{-} \pi^{-} \pi^{+} \pi^{+} \pi^{0}$ & $1.9 \pm 0.4$ \\
\hline
\end{tabular}

gtep telly wa that even if we can reconstruct every last $D^{\mathbf{0}}$, only $7 \%$ of all charm events will fall into our event sanple using this technique if we make no ofher cuts. Unfortunately, we need to impose inore cuts, and only a snell fraction of $D^{0}$ decays are simple enough to be reconstructed in a straghtixward manner. In Thble 2.5 we liat tbe largest branching fractions of the $D^{0}$ for reference[66].

In general one woukd choose to concentrate effort on the $D^{0}$ clecay modes with the largert branching fractions to maximize the statistical power of the redult. As mentioned above, however, the cleareat signals are obtained when one call wo both the reconotructed $D^{\circ}$ maes and the $\Delta m$ diatribution, co urodes with invirible neutrah we difinored. Since the inefliciescles involved in $\pi^{0}$ reconseruction are often lerge, the 
modes exweninis wore thes one $x^{0}$ aloo sre often excluded. The special kinemstics of the decay $D^{0}+K^{-} \pi^{+} \pi^{0}$ make it suitable for inclusion sirce the $\pi^{0}$ leed not be reconstructed is obtein a nartow mas peak aritable for eveut selection. This comes abrout in the following manner[65]. The decay $D^{0} \rightarrow K^{-} \pi^{+} \pi^{0}$ can proceed through two intrrmediate resunance decays $D^{0} \rightarrow K^{-} \rho^{+}$and $D^{0} \rightarrow K^{+-} \pi^{+}$. Since the $D^{0}$ in a peixdoscalar, these decays are both from a state of $J=0$ to states of $J=1+J=0$. In this rase, the vector meson must bo in the $J_{z}=0$ state to conserve angular montentum. This results in the decay products being emitted jreferentially aiong the vector meson direction $\left(m_{,}, 0 \times 00 \theta\right)$. In exch case, approximatoly helf the time, the $K^{-}$and tlie $\pi^{+}$will be etnitted in opposite directions in the $D^{0}$ rest frame, which results in their combination laving a high invariant man. The other $\sim 50 \%$ of the conbinations ylelds a peak at lower nans. The peak is sufficiently narrow for one to select these decays by impresing a mass cut in a band lekow the true $D^{u}$ mass. Typically, then, the decay ankies $D^{n} \rightarrow K^{-} \pi^{+}, D^{0} \rightarrow K^{-} \pi^{+} \pi^{0}$, and $D^{0} \rightarrow K^{-} \pi^{+} \pi^{+} \pi^{-}$are iwluded in audyes which attenpt to isolate n mample euriched in primary clasin events using exclusive decay modes.

The fraction of charin evente remaining after each step in the decay of a charm quark for these devay modics cen be summarized by

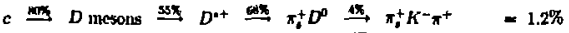

$$
\begin{aligned}
& \text { 䏚 } \pi_{1}^{+} K^{-} \pi^{+} \pi^{0}=4.2 \% \\
& \stackrel{8 \pi}{\longrightarrow} \pi_{0}^{+} K^{-} \pi^{+} \pi^{+} \pi^{-}=2.4 \%
\end{aligned}
$$

where the branching frwetions for $a \rightarrow b$ are given above the arrow for each step in the ceacmbe. So, the wel charm-sclection efficicucy of these analywes could be as high as $8 \%$ if miltiple decay modks aro included (assuming $100 \%$ event belection efficiency; typical event melection efficiuncien are as high af 20\%). Event samples derived with these selection teciniques have also been ued at LEP $[67,68,69)$ to ineaure $A_{F B}$. asuming $A_{F B}^{*}$ is given by its standard model value. 


\subsubsection{Lifetime The Combined with Jet Charyo}

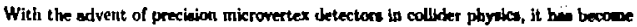
possible to reliably separate events containing heavy hadrons from thowe with only light hadrous by uing the lifetime signatures of the decaying hadrow. Heavy, woldydecaying hadrons such a $B$ and $D$ meson huve "lanf" lifetimes on the order of 1 pe, which, at Z0energies, implies that thelr average decay lengths will be one to several millimetera. Their decay products will teud to have large $p_{\perp}$ relative to the meeon Alight direction, which malues the secondery decny vertices cusily resolvable wing a vertex detector whose typicial impact paraneter resolution is thw than $100 \mu \mathrm{m}$ for these trucks. Heavy quark events can be tagget in an inclusive unanher by requiring the preseuce of a decay vertex or vertices ith an event or hernisphere that is well separated from the interaction point. Requiriog some numbrr of tracks to appear not to originate from the interaction point, either by simple counting or by a libelihood function busel on this information, can aloo he tuadl. A lifetime-aignificance libel. bood function tag was used to measure $\Gamma\left(Z^{0} \rightarrow b \bar{b}\right) / \Gamma\left(Z^{0} \rightarrow\right.$ hadron). in Ref. [70]. Up to this tirte, tags of this type have concentrated on isolating pure samples of b hadrons, as their typkal lifetiunes and decay snultipticitics are largent. Ericiencies in axcess of $60 \%$ for purities above $90 \%$ have bren achicked for these surts of tags when they are applied to select $b$ events.

Once a puro sainple of $b$ eveuts (in this case) has bren selected, an inclusive way mist be found to mesure the charge of the ontgoing finarks to discern whether the fernion was producet in the forward ix barkward hemisplucre. One way to prosed ja to employ the moxnentum-weighted jet charge $Q[71]$ :

$$
Q=\sum_{\text {tracts }}-q_{h} \cdot \sin n\left(\vec{p}_{i}-\hat{T}\right)\left|\vec{p}_{i} \cdot \hat{I}\right|^{n} .
$$

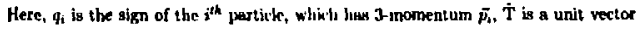
along the tlurust axis direction, and $\kappa$ is un adjustable parameter which muat be optimized for each situstion. The sign of the vector $\dot{T}$ is chosen guch that overall oum $Q$ is neystive, making $\mathrm{T}$ wi estimate of $t \mid \mathrm{se} b$ quark direction. $Q$ w defined auch tinat

\footnotetext{
For dealle ol a slaple coupting tas, wo Chapher?.
} 
high momentutn particles close to the thrust win contibute the rrost information to the charge aum in an attempe to meximize the weight of the $b$ decay product in the sum. This is succesoful since the hard $b$ quark fraganentation function results in the decay products of the $b$ having large average momentum along the original $b$ quark direction in andition to carrying the majority of the enersy in each thrust hetnisphere. The LEP experiments have measured $A$ ks using thin technique[?]2].

\subsection{Measurements of $A_{b}$ and $A_{c}$ at SLD}

The anplysis that is presented in this theris is a single panel in a triptych of measure ments of the parity-violating couplings $A_{4}$ and $A_{c}$ performed using the SLD detector at SLC and the techniques described above.

Using the 1993 data sample of $Z^{0}$ decnys, measurements of $A_{b}$ and $A_{e}$ have been performed using high-p muons and electrons a a tag of heavy quark events. As an illustration of the results, the asymmetry ws. the poler angle of the outgoing fermion is shown in Figure 2.13 for leptons scletted with the elliptical cut of Eq. 2.88. The

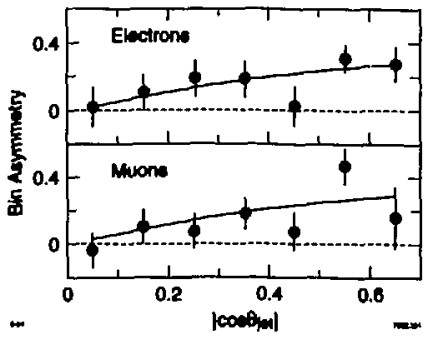

Figure 2.13: The left-righe forward-backward asymmetry for high $p, p_{\perp}$ leptons. 


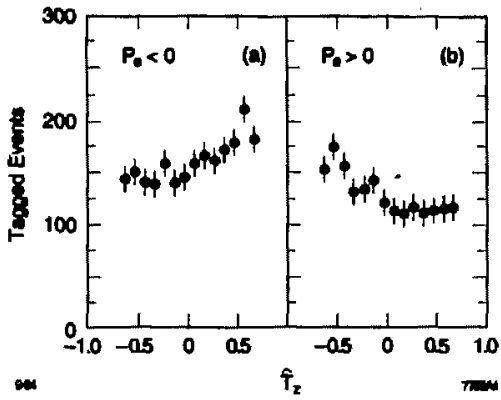

Figure 2.14: The outgoing $b$ quark direction as determines by jot charge for the two beam belicities. Note the large raw asymmetry.

curve drawn through the points is the asymnetry function from Eq. 2.72. Note that the obwerved asymuktry is quitc large, as would be expected with the large abeolute beam polarization (63\%) of this data set. Froul a unairnum likelihood analyais of the lepton $p$ und $p_{+}$spectra, they obtained the following rosultei[73] for $A_{6}$ and $A_{c}$ :

$$
\begin{aligned}
& A_{b}=0.01 \pm 0.14 \text { (stat) } \pm 0.07 \text { (syst) } \\
& A_{\varepsilon}=0.37 \pm 0.23 \text { (stat) } \pm 0.21 \text { (syst) } .
\end{aligned}
$$

Note that the comment made above in reference to the typical crtor size on $A$ f 8 from uneaurements using the lepton tac techique applies equally well to mewarenents of $\boldsymbol{A}_{\boldsymbol{c}}$.

A jet-charge technique has deo been employed to measure $A_{b}$ atter a mapple of b events whes selected wing at inpect parameter tayging technique like that deceribed in Clapter 7. To dernonatrate the power of this technique, we ahow in Flare 2.14 
the raw dientibutions of the poler ande of the outgoing fernilon for the two diffrent bangitudinal polariantion states of the beam. The raw noymmetry is quite large, esen if the ideneification of the fermion direction is not quite perfect" ". A bisned tit to the expreation in Eq. 2.72 afler backgrond suberaction yields[74]

$$
A_{4}=0.87 \pm 0.11 \text { (stat) } \pm 0.09 \text { (syst) . }
$$

All of the above results hre consiatent with tile standard model values of $A_{4}=0.93$ and $A_{c}=0.67$. Comparison of thes value with those obteited th the LEP collaborations will be nuade in Cliapt:r 1.

We now turn to s description of the metlods used in this thesis to meseure $A_{c}$.

\subsection{The Mensurement of $A_{c}$ Using Exclusively Reconstructed $D$ Mesons}

In this thesis, we cotwentrnte on a measurcment of the parnmeter $A_{c}$ uging anl enriched mmple of $Z^{0} \rightarrow$ ã crents. As discused bbove, the only way such a sample can be obtained is through the reconst ruction of exclusive decay channels of $D$ means, and this will be our method. Since the charm-tageing efficiency of this method is so small and we begin with a data gample 50 times smaller than that of the LEP experiments, we weed to rlesign on analysis that yiekls as many charm events wos posible in order to arrive at a conjpetitive meawrenetr for $A_{c}$. To this end, we have created a new met of sekection criteria for isolating $D^{0}$ cecays that make extensive use of the SI,D precision vertex detector by reyuiring that the those particles used to reconstruct the $D^{\text {a }}$ nueson also form a sccondery decay vertex well separated from the interaction point. The senpie of $D^{4}$ decays used to meanure $A$ is a combination of events belected ly the stuxderd kinematic annlysis and thowe chowet by the new decay length melection tritcris. In addition, to maxinize the statintical power of the neagurement we include a xample of $D^{+}$decays. Note that sign of the $D^{+}$also unatobigueusly determines the sigl of the charm quark produced in the $Z^{0}$ decay. Sinec there is no

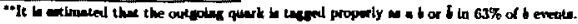




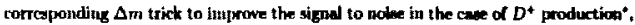
we are snly intereated in thowe $D^{+}$secay modes with a lerge probubility for correct

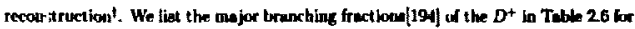
comparison to Table 2.5; we heve choeen to consilder only the mode $D^{+} \rightarrow K^{-} x^{+} x^{+}$ for this andysis at it offers a large branching fruction wid containe no $\pi^{2 / b}$. The $D^{+}$ sclection analysis nlso makes extensive use of the vertex detectur to obtein a smple of events with very little beckground.

A detaikd description of the $D^{\infty}$ and $D^{+}$selection lechminges will be presented in Chapter 13. Discuswion of the likelihood fitting nethod used to cxtract $A_{c}$ follows in Chapter : with a discussion of systematic errors on the axeasuretuent (Chapter 1) und conclusions (Chapter 1) serving to close thez electrowenk part of this thesis.

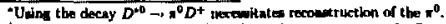

TOre udvatage ta uing the $D^{+}$unowke in that the prosence of a $D^{*} \rightarrow D$ cancade la not required.

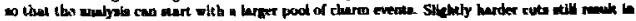
- lerto matpip of events 
Table 2.6: The largest branching fractions in $D^{+}$decay. We show here the brankhing fractions into topological hinal states ignoriug any resonent aulsetructure.

\begin{tabular}{|lc|}
\hline Dechy Mlode & Branching Fraction (\%) \\
\hline Seni-kptonic Modes: & \\
$D^{+} \rightarrow K^{0} e^{+} \nu_{c}$ & $6.6 \pm 0.9$ \\
$D^{+} \rightarrow K^{0} \mu^{+} \nu_{\mu}$ & $7.0_{-3.0}^{+3.0}$ \\
$D^{+} \rightarrow K^{-} \pi^{+} e^{+} \nu_{e}$ & $4.2_{-0.7}^{+0.7}$ \\
$D^{+} \rightarrow K^{-} \pi^{+} \mu^{+} \nu_{\mu}$ & $3.2 \pm 1.7$ \\
Hadronic Mlodes: & \\
$D^{+} \rightarrow K^{0} \pi^{+}$ & $2.74 \pm 0.29$ \\
$D^{+} \rightarrow K^{-} \pi^{+} \pi^{+}$ & $9.1 \pm 0.6$ \\
$D^{+} \rightarrow K^{0} \pi^{+} \pi^{0}$ & $9.7 \pm 3.0$ \\
$D^{+} \rightarrow K^{-} \pi^{+} \pi+\pi^{0}$ & $6.4 \pm 1.1$ \\
$D^{+} \rightarrow K^{0} \pi^{+} \pi^{+} \pi^{-}$ & $7.0 \pm 1.0$ \\
$D^{+} \rightarrow K^{-} \pi^{+} \pi+\pi^{0} \pi^{0}$ & $2.2_{-0.0}^{+3.0}$ \\
$D^{+} \rightarrow K^{0} \pi^{+} \pi^{+} \pi^{-} \pi^{0}$ & $5.5_{-1.0}^{+3.0}$ \\
$D^{+} \rightarrow K^{0} K^{0} K^{+}$ & $3.1 \pm 0.7$ \\
\hline
\end{tabular}




\section{Chapter 3}

\section{The Strong Interaction: An Introduction}

The quest for the correct theoretical formulation of the muclear force bus continued since the clerlficution of alclear slucture that resulted from Chadwich's discovery of the neutron in 1935 [75]. Yukewn's pion [76], ahthough it provided a key concept in the sevelopment of fiekd theory, proved to be the first of an enorinous number of meson states instead of the particle solely responsible for cartying the strong interaction. The incredible simplification realined in the quirk nodel of Gell-Mann and Zweic [ $n]$ and the introduction of "color" by Han and Nambutrol represent the comerstone of oar "modern" upderstanding of strong interactions, as their supposition of flavor and color $S U$ (3) as the cortect group sylunetries has been borne out by experiment. The early 1970 's nitnessed the trassition of quarks from phantoms to physical entitles [79] in the deep-inelastic electron scattering experiments performed at SLAC. The disconery[80] that the non-A helian nat urc of Yang-Mlills theories[10] alkowed the ultraviolet freechon of quarks necesary to expluin the Bjoricen scaling sect in these experinents allowed the theory of the strong inturactions that we now know as Quantum Clirenodynamica (QCD) to resch its final form. The obecrvations at PETFA [8t] of jets of particles iuterpreted to be from the radiation of hard gluons served to cement QCD as the "correct" theory of the strong interactions. Since that titne, all other tests of the axtimptions of QCD have reinforced this conclusion. Since part of the topic of thic 
thesis is another of the teots of QCD, it is proper to review in more detnil the theoreticni formulation of QCD and the manner in which it can be teated.

\subsection{Quantum Chromodynamics}

This section presents a discussion of the theory of the strong interactions, proceeding from its basic grousp-theoret ical symmitries through the question of remormalizability. The phenomerological stccesses of $Q C D$ will by pointed out at the appropriate points.

\subsubsection{The QCD Lagrangian}

We begin with the lagrangiau density for Quantum Chromorlynamics* of one quark field $q$ :

$$
\mathcal{L}=\bar{q}\left(i \gamma^{\mu} \partial_{\mu}-m\right) q-g_{\rho}\left(\overline{\bar{q}} \gamma^{\beta} \mathrm{T}_{*} q\right) B_{\mu}^{\alpha}-\frac{1}{4} C_{\mu \nu}^{*} G_{\alpha}^{\mu \nu} .
$$

Hete, $T_{*}(a=1, \ldots 8)$ are the generators of $S U(3), B_{n}^{n}$ are the eight gange-invariant cluon fieldy, and $g$ is the coupling introduced in requiring lorel SU(3)-color gauge inveriance. The generators $\mathrm{T}$ sutisfy

$$
\left[\mathrm{T}_{a}, \mathrm{~T}_{b}\right]=i \mathrm{f}_{\mathrm{ar}} \mathrm{T}_{\mathrm{c}}
$$

where the factors $f_{\text {alc }}$ are the $S U(3)$ structure constants. The kinetic energy term is given by

$$
C_{\mu \nu}^{a}=\partial_{\mu} B_{\nu}^{\alpha}-\partial_{\nu} B_{\mu}^{\alpha}-g_{\nu} f_{\Delta \alpha} B_{\mu}^{+} B_{\nu}^{*} .
$$

This lest term, required by local geuge-invariance, seperates the behaviour of QCD from that of Quantum Electrodynamica (QED), as it implies that the gluons themselves carry the color charge. This addition arises due to the non-Abelian uature of the gauge group; similer terns occur in the Electromeak Lagrangian, Eq. 2.2. The interactions amociated with ench of the terms in the Lagrangian of Eq. 3.91 are shown *hematically in Figure 3.1.

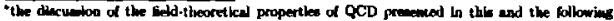

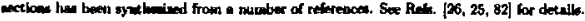




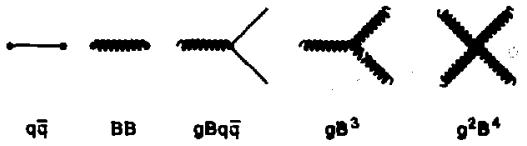

Figure 3.1: A symbolic representation of the interactions contuined in the QCD 2 grangian. The diagrams labeled $q q$ and $B B$ are the quark and gluon propmegtors. The vertices to order $g$ are shown next, and finally, the four-gl'ton vertex is shown.

It lans become customary to refer to the dinutity $a_{3}=x_{12}^{2}$ ws the strong coupling. All further discussion of the strong couphing in this thesis will follow this convertion.

\subsubsection{Simple QCD Procene:}

In this bection, we will diecure the processes $\mathrm{e}^{+} e^{-*} \rightarrow q \hat{q}$ and $e^{+} e^{-} \rightarrow q \bar{q} g$ in order to extibit sone of the propertics of the strong interaction formulated in QCD. Figure 3.2 a siows the Faynan dingram for $\mathrm{c}^{+} \mathrm{e}^{-} \rightarrow a \bar{y}$ ut the tree level, whit in except for the required fuctor of $N_{c}$ (the number of colors) in the croes section, recudires no input from QCD'. To lowest order is the strong cuuplitg $\alpha_{s,}$ the rate for $\mathrm{e}^{+} \mathrm{e}^{-} \rightarrow q \bar{Q}$ is modifier by virtual corrections due to internal gluson loops, as shown in Figure 3.2b. Each of these diggramus contains ultra-viojet and/or infra-red divergences, but the sum of the squared amplitudes yiekda a finite result for the total croses section when the $\mathcal{O}\left(a_{2}\right)$ correction dwe to the enirsion of a real gluon (Figure 3.3n) is adked. For completeness, Figure 3.2c contains the Feyrinan graphs of proceses of order $a_{\text {a }}^{2}$ incluched in a higher order ralculation of $e^{+} e^{-} \rightarrow q \bar{q}$. The schlition of these dingrams to those for sll other $O\left(a_{*}^{2}\right)$ processes, including those in Figure $3.3 \mathrm{~b}$ and Figure 3.4 also results in a finite total cross scetion for $e^{+} e^{-}-$hudrous after the coupling constant is renormalized (aee the next section).

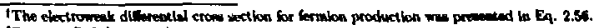

'See, e.g., Ref. \{62, p. 50\}. 
(a)

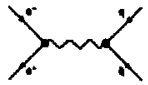

(b)

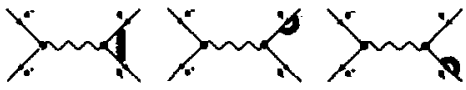

(c)
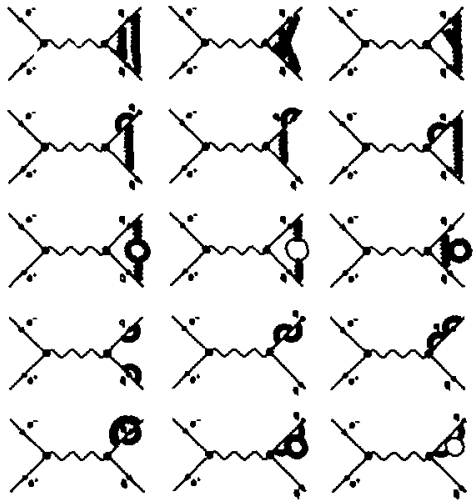

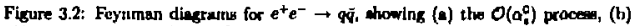
$O\left(a_{1}\right)$ processes, and $(c) O\left(a_{n}^{2}\right)$ procenes. 
(a)

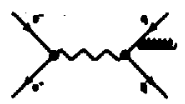

(b)
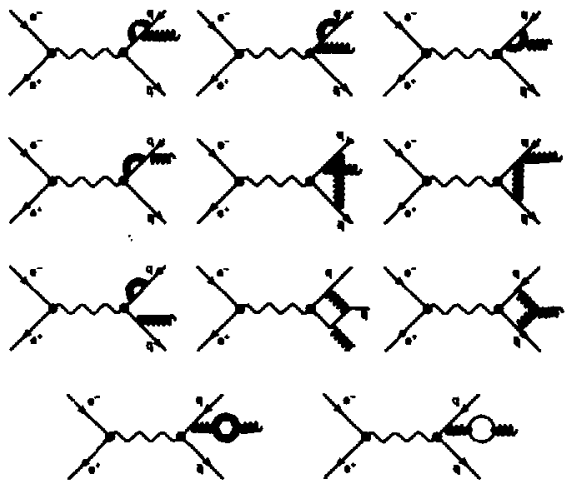

Figure 3.3: Feynmen diagrams for $e^{+} e^{-} \rightarrow q \overline{q g}$, showing (a) the $\mathcal{O}\left(o_{3}\right)$ process, and (b) $\mathcal{O}\left(a_{2}^{2}\right)$ jrocesses.

Lot us consider explsitly the process $e^{+} e^{-} \rightarrow$ wig where, instead of being inkerested only in the total cross section for $\mathrm{e}^{+} e^{-} \rightarrow$ hadrons, we would like to obeerve the radiated gluon. To howest order in QCD, the differential crom noction for radiutine a 

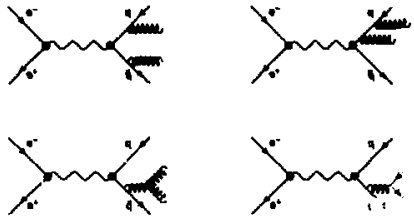

Figure 3.4: Feymman diagrams for $e^{+} c^{-} \rightarrow q d q 9$ and $e^{+} e$

single gluon in exents of the type $e^{t} c^{-}-q \bar{q} g$ (sec Figure 3.3a) is given by|Bi3|

$$
\frac{d \sigma}{d x_{1} d x_{2}}=\sigma_{0} \frac{2}{3} \frac{a_{1}}{\pi} \frac{x_{1}^{2}+x_{2}^{2}}{\left(1-x_{1}\right)\left(1-x_{2}\right)},
$$

where $\sigma_{n}$ is the lowest order Born cross section,

$$
\theta_{0}=\frac{4 \pi \alpha^{2}}{3 s} N_{c} \sum_{l} q_{j}^{2},
$$

and $x_{i}=2 E_{j} / E_{G N}$ for $i=1: \vec{q}, i=2: q, i=3: g$. Here, $N_{c}=3$ is the number of colors, $\alpha$ is the electroneak coupling, and $q_{f}$ is the clange of quark flavor $f$. Of course, the values of $x$ tlest are kibenstically allowed in this formulation are $0 \leq x \leq 1$.

The lowest-order crows section of $\mathrm{E}_{\mathfrak{l}}$. 3.94 diverges as $x_{1}$ or $I_{2}$ spproaches unity. The caso $x_{1} \rightarrow 1, x_{2} \rightarrow 1$ rorresponds to the emiseion of a gluon of vanlshingly small momentum. This is an infrared divergence, in that the croes section diverges when tiwe momentuy of the emitted quantum approaches zero. The other case, where $x_{1} \rightarrow 1, x_{2} \neq 1$ corresjonds to the situation where one of the quarks emits a soft, collinear gluon, and is known as a collinear divergence. In either case, tle gluon is not rewolvable frout the $y$ or $y$. As stated ubove, however, the total crom nection for $\mathrm{e}^{+} \mathrm{e}^{-} \rightarrow$ loudrons is finite, wo the probability of radiating a "risolvelble" final-state glwon must also be finite. We can define the term "rewolvable" by any number of ulferent criterin. For exauple, the energy of the outgolng gluon and ita andea with 
respect to the two quarks must be ulwow sotne threshold [84], or, equivalentiy, the scaled invariant maw xpluared

$$
y=\frac{m_{i j}^{2}}{1}
$$

must be grenter than some resolution paraintict $y_{\text {min }}$ for ull pmirs of quarks and gluons[85]. Integration over the region of pluse space that the plinos is reolvalste yields a 'inite croes section for $e^{+} e^{-} \rightarrow q \bar{q} g$ events'. Note that the croses section for

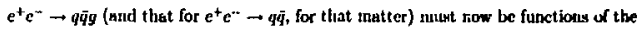
resolution critcris[89];

$$
\begin{aligned}
& \sigma^{4 q}\left(y_{\min }\right)=\sigma_{0}\left[1+\frac{4}{3} \frac{\alpha}{2 \pi}\left(-2 \log ^{2} y_{m 1 n}-3 \log y_{\min }+4 y_{\min } k_{g} y_{\min }-1+\frac{\pi^{2}}{3}\right)\right] \\
& \sigma^{a+s}\left(y_{\min }\right)=\sigma_{0} \frac{4}{3} \frac{a_{1}}{2 \pi}\left(2 \log ^{2} y_{\text {min }}+3 \operatorname{kg} y_{\text {min }}-4 y_{\text {min }} \log y_{\text {min }}+\frac{5}{2}-\frac{\pi^{2}}{3}\right)
\end{aligned}
$$

Note that the cross section for the threaperton fual state is proportional to $u_{\text {ai }}$; we will use the measurenkent of this quantity to detive the magnitude of the gtrong coupling.

Similar chl:ula wons have been performed to oltain mat rix elenents for more coulplicated finul vintes of quarks and gliusus (henceforth suferred to collectively as "partons"). In particuler, results cxist fur the cross sections for four $\left(e^{+} e^{-} \rightarrow q \bar{q} g g\right.$ or

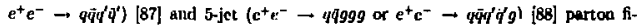
nal states. In prinriple, these calculutiong riun be comparel with the experimental mcasurements of the same quantitits us a check of the volidity of higher-orier QCD caleulations.

As mentionel sbove, the individual feyman amplit udas for the first order QCD corrections to any process contuin divergences. Since the "bawe" corpling of the theory appears to le: infinite when the liryt orcher corrections are ackld, it is useful as u comput ationtal tool to invoke a revunnalizafion procedure to redefine the coupling a, us a finite experiment ul olwervable aud ebeorb the infinitins of the individual grapilw. This will be discuwarl in the sext section.

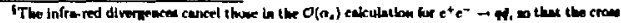

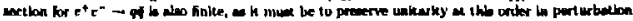
theory $[80]$. 
(a)
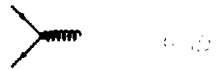

$-\log _{0} \gamma_{p} \mathbf{T}_{.}$

(b)
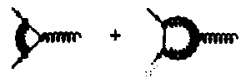

$-\log _{0} r_{\mathbf{F}} T_{0}\left(z_{1}^{-4}-1\right)$

(c)

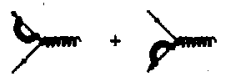

$-2\left(z_{2}^{\prime}-1\right)\left(-b_{0} r_{1} T_{0}^{\prime \prime \prime}\right.$

(d)
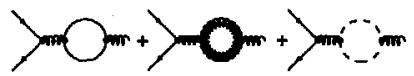

$-D_{0} \gamma_{\mu} T_{1}\left(Z_{3}-1\right)$

Figure 3.5: The legding order corrections to the quark-gluon vertex, i.e., to $\alpha_{11}$ showing: (n) the bere 999 vertex; (b) the one loop vertex correction, which multiply the bare vertex by the factor $\left(Z_{1}^{-1}-t\right)$; (c) the quark self-energy correctlous, which multiply the bare vertex by the factor $-2\left(Z_{2}^{-1}-1\right)$; (d) the gluon self-energy corrections, which multiply the bare vertex by the factor $\left(Z_{3}-1\right)$. The solid lines corropond to quarks, the lielical curves represent gluons, and the dashed lines are the "ghost" distribution acessary for renoving non-physical gluon polarization stetes from the calculation.

\subsubsection{The Renormalization of the Strong Coupling}

Before moving on to a diseussion of the various predictions of QCD, the behaviour of the theory at the one-loop level needs to be explozed. It is the renormalized coupling which exhibits all of the salient fenttures of QCD phenomenology, and we turn now to a diseussion of its derivation.

The lowest ordet diagrams and virtual corrections to the quark and gluon propagators are shown in Figure 3.5. As is shown in the figure, the lowest order vertex factor $-i g_{,}^{0} \gamma_{\mu} T_{\mu}$ is modified" by the various one-loop corrections:

\footnotetext{
TExpresions for the corrections can be fouid in, e.g. Ref. [82]. Nole thas Firure 3,5d contains an oxtra diagrarti due to the "ghoot" terius wlitch mut be addexl to remme non-physical pulariration teres in the gauge chosen for thit calculation.
} 
- $-i g_{\mathrm{s}}^{0} \gamma_{\mu} \mathrm{T}_{\mathrm{a}}\left(Z_{i}^{-1}-1\right)$ by the vertex costections,

- $\left(-i g_{\mu}^{0} \gamma_{\mu} T_{a}\right)(-2)\left(Z_{2}^{-1}-1\right)$ by the quark self-enetcy correctiotus,

- $-i g_{0}^{0} \gamma_{\mu} \mathrm{T}_{\mathrm{a}}\left(Z_{3}-1\right)$ by the corrections to the gluon propagator.

Each of the $Z_{1}$ terns contain uitra-violet divergences resulting from the infinite momenta which are posible in the parton loops. Thew infinities must be sboorbed into an effective cospling constant in order to render the theory calculationally weful. Including the proper normalization for oxternal fermion lines and the gluon cource, we can odd the anplitudes for al of the diagxass to arrive at

$$
\begin{aligned}
-i g_{.} \gamma_{\mu} T_{4} & =\frac{-i g_{n}^{0} \gamma_{\mu} T_{n}}{Z_{2} \sqrt{Z_{3}}}\left[1+\left(Z_{1}^{-1}-1\right)-2\left(Z_{2}^{-1}-1\right)+\left(Z_{3}-1\right)\right] \\
& =\frac{Z_{3} \sqrt{Z_{3}}}{Z_{1}}\left(-i g_{\mu}^{0} \gamma_{\mu} \mathrm{T}_{4}\right)
\end{aligned}
$$

or

$$
y_{2}=\frac{Z_{2} \sqrt{Z_{3}}}{Z_{1}} g_{1}^{0} .
$$

This coefficient can be written as a power saries in terms of $a^{0}$, the bare coupling, where $\alpha_{0}^{0}=g_{0}^{0} / 2 \pi$. The llew, renormalized effective coupling $\alpha_{s}^{\text {eff }}$ is given by

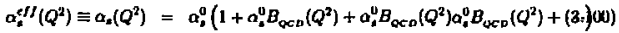

$$
\begin{aligned}
& =\frac{\mathbf{o}_{\alpha}^{0}}{1-\mathbf{a}_{0}^{0} B_{\alpha c D}\left(Q^{2}\right)} \text {. }
\end{aligned}
$$

Here, $B_{\text {qCD }}$ is the actunl functional form of the result, nud $Q^{2}$ is the gluon momentum wyuared and hence the energy avxilable for the intersetion. In general, the specific form of $B_{\text {OCD }}$ depends on the nethod chosen to regulate the divergences that arize in calculating the $Z_{i}$ factors while preserving gauge invariance. One way to do this, called dimensional regularization[90, 91] is to ralculate the loop diugrains in $N$ dimengions, where $N$ is typically set to $N=4+\epsilon_{\text {r }}$ and then take the limit of the results as $N \rightarrow 4(e \rightarrow 0)$.

Equation 3.101 implies that

$$
\frac{1}{\alpha_{n}\left(Q^{2}\right)}=\frac{1}{a_{0}^{0}}-B_{Q ⿻_{C D}}\left(Q^{2}\right) .
$$


The function $B_{\text {Qco }}\left(Q^{2}\right)$ diverges, however, and a stanclard renormalization procedure must be folkowed in order to obtnin a finite, obecrvable coupling in terins of a finite difenence of intinitc terms. Since $B_{Q C D}\left(Q^{2}\right)$ is propertional to $\log Q^{2}$, we caunut define the coupling in the linit of xero nomentum transfer. Instead, we inust choowo a finite reference an:sle $Q^{2}=\mu^{2}$ and defino the "experimental coupling" as

$$
\alpha_{2} \equiv \alpha_{1}\left(\mu^{2}\right)
$$

as a replacement for $a_{d}^{0}$ where it appers. The relntionship betwent the experimental compling and the bere coupling is given, then, by

$$
\frac{1}{a_{0}^{0}}=-\frac{1}{\mu_{a}\left(\mu^{2}\right)}+B_{\alpha C D}\left(\mu^{2}\right) \text {, }
$$

so that at other values of $Q^{2}$, we find the coupling is

$$
\frac{1}{a_{s}\left(Q^{2}\right)}=\frac{1}{a_{s}\left(\mu^{2}\right)}-\left(B_{Q C D}\left(Q^{2}\right)-B_{Q C D}\left(\mu^{2}\right)\right) \text {. }
$$

Sitce the $Q^{2}$ defendence of $H_{Q C D}\left(Q^{2}\right)$ only ajppears in a logarithmic term,

$$
B_{Q C D}\left(Q^{2}\right)-B_{Q C D}\left(\mu^{2}\right) \propto \log \left(Q^{2} / \mu^{2}\right) .
$$

To lowest order [82, p. 242], the exact expression is

$$
B_{Q C D}\left(Q^{2}\right)-B_{Q C D}\left(\mu^{2}\right)=-\frac{\beta_{0}}{4 \pi} \log \left(Q^{2} / \mu^{2}\right),
$$

where

$$
\beta_{0}=\frac{11}{3} C_{A}-\frac{4}{3} C_{F}=11-\frac{2}{3} n_{s} .
$$

Here, $C_{A}$ and $C_{F}$ are the Dynkin indices of the adjoint artu fundamental representutions of $S U(3)^{4}$, and $n_{f}$ is the pumber of quark flavors avcesejble at energy rang: $Q^{2}$. The difference in the two infinite $B_{\text {oco }}$ tertns is finite and doesn't depend on

\footnotetext{
"Thne factors arles in calculating the traces in obtainging tlx bop amplitudes. The quantity

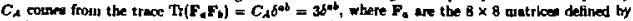

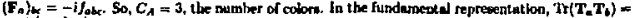

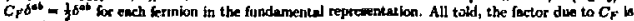
given by $\frac{1}{2} n_{f}$. wlicere $n_{f}$ wa the number of acceenibls kermilows.
} 
the contants introduced by dinchwiounl regularleation. Insertlng this expresulou into Equation 3.104 yidids

$$
a_{s}\left(Q^{\mu}\right)=\frac{\alpha_{s}\left(\mu^{2}\right)}{1+a_{s}\left(\mu^{2}\right) \frac{\alpha_{4}^{S}}{4} \log \left(Q^{2} / \mu^{2}\right)} .
$$

It is cossunon to rewrite the above equation in terms of a single parameter, often denoted $\boldsymbol{\Lambda}$. If we make the replacenent

$$
\log \left(\Lambda^{2}\right)=-\frac{4 \pi}{A_{0} \omega_{n}\left(\mu^{2}\right)}+\log \left(\mu^{2}\right),
$$

we cun rewrite $\mathrm{E}_{\mathrm{i}} .3 .108$ as

$$
\alpha_{s}\left(Q^{2}\right)=\frac{4 \pi}{\beta_{0} \log \left(Q^{2} / \Lambda^{2}\right)} .
$$

This perticular cheice of which constauts are absorbed into $\Lambda$ is known as the Minimal Subtraction (MS) scheme[92]. Other schenves, such as the Modified Minimal Subtraction (MS) scheme|9J], also exist.

Note that there two effects of the renormatization of the coupling. Fint, the coupling $a$, now is fuuction of $Q^{2}$, i.e. $\alpha$, is a rwnning coupling. Second, the renormalization procedure has re-expressed the coupling of QCD as a function of a single parameter, $\Lambda$, which is now the funduriental parameter of QCD.

\subsubsection{The Altarelli-Parisi Dquations: The Leading Logarithm Approximation}

It was shown by Altarelli and Parisi[9]] that, to leading order in powers of the logarithms $\log Q^{2} / \Lambda^{2}$, the resules of $\mathcal{O}\left(\alpha_{*}^{2}\right)$ QCD calculations for the behaviour of the Btructure functions in derj-inelastic scattering can be reprodiced by a consideration of only the basic vertices of QCD coupled with parton probability densities in an infinite momentum frame $\left(p \rightarrow m_{4}\right)$. Their description of hard scattering is valid to all orders in $a_{,}\left(Q^{2}\right) \log Q^{2} / \Lambda^{2}$. These corrections to the tree level diagrume can be wurnmed to intinite order, resulting in the so-called Leding Logarithm Appraximation (LLA)[96] to QCD. 
The Alterelli-Perisi fornulation of the LLA in terms of the more intuitive parton model enneepts allows a unuch simpler besis for calculating thooc quantities where the loading logurithms add importunt contributions. In thejr deacription, the basic vertike of QCD are expreseed in terms of probability densities fox the branching of one parton into two partons, $P_{a-k}$. The evolution of a parton deneity function $f_{t}\left(z, Q^{2}\right)$ is given by

$$
\frac{d f_{0}\left(z, Q^{2}\right)}{d t}=\frac{a_{*}\left(Q^{2}\right)}{2 \pi} \int_{2}^{t} \frac{d y}{y} P_{n \rightarrow b_{c}}(y) f_{6}\left(\frac{z}{y}, Q^{2}\right) .
$$

Herc, $t$ describes the evolution of the system, e.g. $t=\log \left(Q^{2} / \Lambda^{2}\right)$, and $z$ gives the fraction of the initial partion exergy retained by parton $b^{*}$. The $\left(Q^{2} \text {-inclependent }\right)^{\text {th }}$ functions $P_{a-b e}$ uxe given by:

$$
\begin{aligned}
& P_{1-\infty}=\frac{4}{3} \frac{1+(1+z)^{2}}{z} \\
& P_{g \rightarrow \infty}=6\left(\frac{1-z}{z}+\frac{z}{1-z}+z(1-z)\right) \\
& P_{g \rightarrow \infty}=\frac{41+z^{2}}{3} \frac{}{1-z} .
\end{aligned}
$$

The uscfulness of this formulation in the generation of purton distributions within a MC simulation of hadron production will be discussed below in Section 3.2.3.

\subsubsection{Higher Order Corrections:}

\section{The Renormalization Group Equation}

It is possible to perform the calculation of the renormalized coupling to higher order in $a$, by coscidering the iacreasingly complex Feynmen graphy which oceur. Sozne of these for $O\left(\alpha_{i}^{2}\right)$ art shown in Figure 3.2c.

The results of thewe calculations can be conveniently exprented in terms of the $\theta$ function $\beta\left(\alpha_{n}\right)$ conlained within the so-called Renormalization Group Equation:

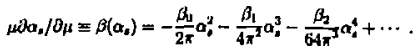

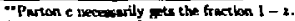

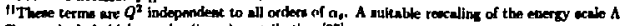

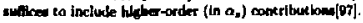


The coefficients are given by

$$
\rho_{1}=\left(306-38 n_{\jmath}\right) / 6
$$

and

$$
\beta_{1}=2857-\frac{6033}{9} n j+\frac{325}{27} n_{j}^{2}
$$

in minimal subtraction (MS) scletred. Note that the definition of the coenicients $\beta_{n}$ for $n \geq 2$ in the series depend on the specific renormalizution procedure uned to ubbtruct the infinities, i.c. they are renormalization-scherne-dependent. The co-called renormalization scale $\mu$ is a mensure of the man glwor enerzy taken in the bigherorder corrections that modify the coupling. It is the same parameter introduced in tite calculation of Section 3.1.3 to define the scale of the leading order corrections.

With this definition of the $\beta$ function, the definition of the scale $\Lambda$ can be given sy

$$
\Lambda=\mu \exp \left[-\int_{0}^{\alpha}\left(Q^{\top}\right)_{A} \frac{d \alpha_{\Delta}}{\beta\left(\alpha_{0}\right)}\right] .
$$

The parameter $A$ is arbitrary and serves to specify the boundary conditions on the choice of scale.

If could perform the calculation to all orders, any dependepce of the anower on $\mu$ must vanish, as it is an artifact of the truncated calculation. We can see from corrbining Eq. 3.113 with Eq. 3.116 that, at least to this order,

$$
\partial \Lambda / \partial \mu=0
$$

though $A$ still depends on the renormalization scheme through the exact, values of the coefficients in the $\beta$ function series.

The integration of the renormalization group equation yields an expreseion for $a$, is tems of $\mu, \alpha$ exuivitenly, from En. 3.116, in terms of $A$. One choice of a reprosentation for this result is[98]:

$$
\begin{aligned}
\sigma_{1}\left(Q^{2}\right) & =\frac{4 \pi}{\beta_{0} \log \left(Q^{2} / \Lambda^{2}\right)}\left[1-\frac{\beta_{t} \ln \left(\ln \left(Q^{2} / \Lambda^{2}\right)\right]}{\ln \left(Q^{2} / \Lambda^{2}\right)}\right. \\
& \left.+\frac{4 \beta_{1}^{2}}{\beta_{0} \log ^{2}\left(Q^{2} / \Lambda^{2}\right)} \times\left(\left(\ln \left[\ln \left(Q^{2} / \Lambda^{2}\right)\right]-\frac{1}{2}\right)^{2}+\frac{\beta_{2} \beta_{0}}{8 \beta_{l}^{2}}-\frac{5}{4}\right)\right]
\end{aligned}
$$


Having proceeded through the discussion of first- aud second-order culculations of the modification of the strong coupling, we stould nause and verify that the coupling we have obteined in relatively surdl, 0 that our use of a power ecries in theos calculations can be abown to be valid, as lesst a posteriori. We would expect that the strong intoraction becomes "atrong" when the enctgies of the quarke are on the order of the binding energy of a simple hadron, like the plon or knon. Inserting a value of $A$ of the order $f_{\pi}(\Lambda=100 \mathrm{MeV} / c)$, we hind that, for valucs of $Q^{2}$ larger then about (5 $\mathrm{GeV} / c)^{2}, \alpha_{n}<0.2$, so at least there is some hope that there is a perturbative regime in whicl calculations based on tle prower series will converge. We wilt now discusto several of the properties exhibited by the renormalized maluc of the strong coupling $a_{*}$.

\subsubsection{Asymptotic Freedom and Quark Confinement}

The renormalized coupling shown in Equation 3.108 or Equation 3.118 posseses a remarkable property: as the momentum transfer of the reaction, $Q^{2}$, approsechto infinity, the strength of the coupling drope to zero[80]. This is kntowr as "Asymptotic Frectom*, and was sunuised to be a necessary part of the thicory of QCD after the

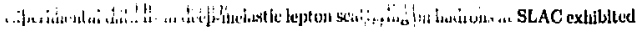
so-called "Bjorken scaling" [99]. The oberviy behaviour of the struct ure functions in

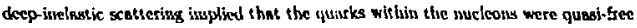
perticles when struck by a hard photon, and thus any theoretical description of the strong force must include this property.

The forn of Equation 3.113 gives us a convenient way to discuss the properties of the theory. Cosubider the lowest order torm

$$
\mu \partial a / \partial \mu \equiv \beta(\alpha)=-\frac{\beta_{0}}{2 \pi} a^{2} .
$$

Since

$$
\beta_{0}=11-\frac{2}{3} n j,
$$

for $n_{j}<16$, the coupling $\alpha$, decreases as $\mu \rightarrow \infty$, i.e., $S U$ (3) QCD is asymptotically free. This was stown to be a general property of Yang-Mills theorits; indeed, only non-Abelien theories have a chasce of being asymptotically free[100]. 
Let us compare QCD and QED. The anakgous cquation to Eq. 3.108 in QED is

$$
\alpha_{E N}\left(Q^{2}\right)=\frac{\alpha_{E N}}{1-\left(\alpha_{E N} / 3 \pi \log \left(Q^{2} / \mu^{2}\right)\right.} .
$$

The coefficient we woukd identify os $\beta_{0}^{O S D}$ is then

$$
\beta_{0}^{a k n}=-\frac{4}{3} \text {, }
$$

which inplies from Eq. 3.119 that the cuupling in QED increases as $Q^{2} \rightarrow \infty$, the diametricully opposite behaviour. It is the countribution of the gluon loops in the gluon sclf-encrgy correction, which are present only because the threengluon vertex exists, that wid the extra, positive tenn in $\beta_{0}^{X C D}$ that makes the thenry arymptotically free.

A more "physical" picture cun be obtained by consideriug $n$ jicture of the "charge strcenis!g" that occurs at stnall distankes in the two theories. Is QED, an electron is surrounded by a cloud of virtunl electron-position poirs. The pairs are polarized in sucls a manuer that the pouitrous are choser to the pote electron, and thus a probe photon of relatively low energy ubeurves a lower, shislded charge, as it canrot reailve the polarized cloud. As the encrey of the photon increases, the distance scale of its interaction shrinks, silowing it to resolve an area containing tewer of the shiplding partickes, and heoce observing a higlwer charge. Something conpletely different happuns in QCD. We can again for simplicity consider a photon probe impinging wa quark of a given color, say bludt. The same vicuum polarization effects that acets in QED will slks be present, i, e., the quark will be surrounded by pairs of blue-anti-blue quarks from the vacuum, which will shield its charge. However, if the blue quark were to emit a virtual gluon of charge, say, biue-nnti-red, it changes for a time into a red quurk. Since this radiation of virtual gluons occur continually, the color charge duc to the quurk is diatributed and diffuse, so that the higher the resolution of the photon probe, the leas effects of the color cbarge it can "sce". In effect, the that that 8 colored gluous cun be radiated overcomes the charge screening behaviour.

If we now look at the opposite $Q^{2}$ extreme, we can set that, w $Q^{2}$ appromches $\Lambda^{2}$

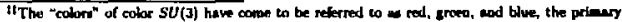
colone of lighe.
} 
in

$$
\alpha_{s}\left(Q^{2}\right)=\frac{4 \pi}{\beta_{0} \log \left(Q^{2} / \Lambda^{2}\right)},
$$

the coupling becomes infinite. Of course, the actud scake at which this happens is somewhat arbitrary, we it depends on the exact value of $\Lambda$ choeen, but it li a genteral fenture of QCD that perturbative calculations are no longer valid when the energy of the quarks and guons approwiches zero $\left(Q^{2} \rightarrow \Lambda\right)$. This behaviour implies that free, individuel, bare quarks are not fonnd in aature. They must be confined [101] within color-neutral hadronic matter, since it wouid requice an infinite amount of entery to liberate them; this is in stark contrast to the behaviour of electrons in the more familiar QED. At the present time, no rigorous proof exista that $S U(3)$ QCD contains confinement. It can be shown[78], however, that the presence of the gluon self coupling results in the lowest-energy states being thowe which are color siuglets. Fo: a discussion of dynarnic symatry breaking in non-abelian theorles and its relationship to confinement, see Ref [102].

The confinement of rusurks and glwons witbin ludrons rewkers the comparison of theoretiral calculations with experimental results difficult. Any experiment designed to test the tenets of QCD must begin and end with oberrvable particles. Regandkos of the energy of the probe used as a measure of the interaction, the parton distributions produced by a procens calculable in perturbative QCD necessarily pass through the incalculable non-perturbative regime as the asymptotically free partons are "dressed" up to become hadrouss which can be obeerved in a particle detector. In practice, experimentaliyts rely on several different Montc Garlo-besed model of this "leadronization" proces in acder to relste the observed distributiony of hadrons back to the urderlying parton proceses responsible for them. The observed diotribistions and the simulation techniques uscd to reproduce them are the suhject of the next section.

\subsection{Production of Hadrons in $e^{+} e^{-}$Collisions}

So fur in this discumion of QCD, whe couceutrated in a somewhst shutrect manner on the feid-theoretical properties of a hadronic theory baad on cotor $s U(3)$ 
and soune general phetwumenologheal implichtions. Here, we premont al overview of hadron production in $e^{+} e^{-}$collwhons inclubding the QCD procenes that realk in the experituental observabsen.

\subsubsection{The Fragmentation Procesw}

As the previous aection implied, when a quark-anti-quark peir is jroduced in the procesw $\mathrm{e}^{+} \mathrm{e}^{-} \rightarrow q d$, the two bare quarks are not otwerved as two individual chargad trackes emerging from the production point. Instewd, a procese simular to that shown in Figure 3.6 occits. The two quarks, slowed by the lincreasing muguitude of the strong

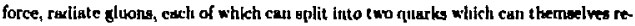
radiate. This "showering" continues until the availntle cuergy is degreded towards $\Lambda^{\mathbf{z}}$. As mentioned above, at this time the coupling becones very large, and the partons are no longer able to exist as free entitiex. $\mathrm{lt}$ is here where the urralculable hadronization procese ckcurs, and we are loft with many differem liedronic states. Many of these states cast be unstable rewonances, which quickly decay into more stuble hadrons. It is this finul distribution of stable hudrons which is obecred in particle detector.

\subsubsection{Jet:}

As will be shown explicitly below, the shower proceses for a single quark is analogous to electromanetic bremsolrahlung. Many of the rmdiated gluons are thus emitted travcling cloee to the initial quark direction. If the priniary quark has sufficlent relativiatic boost, the rediated partons, and hence their lialronic decay products, will form s collimated cono nround the ititial quark mongutuu slirection. These cones of particles are referred to as "jets", und ure ubiquitois in hedronic interactions at high energy colliders $:$. Evidenee for jets was first observed by the MARK 1 experiment[103] at SPEAR, uing an analysis that slowed that the hadronic events becarne keas apherkel (and henee more "jetty") as thu energy of collisions was irerewed from 3 to $7.4 \mathrm{GeV}$. At higher center-of-mass energies, jets are an obvions feature of hadronic evento. In 1979, experianents at PETRA[81] observed planar events with 3 well-beparated jes,

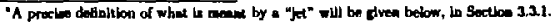




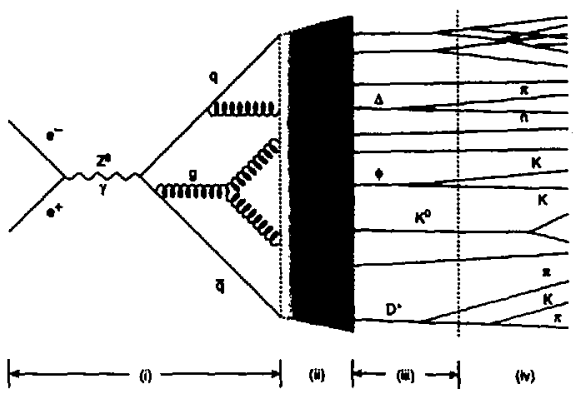

Figure 3.6: An overview of an $e^{+} e^{-} \rightarrow q d$ event. Four stages of the event evolution are indicated. Stage (i) is the perturbetive phase, where tive $Q^{2}$ of the interacthons are sutficiently large that perturbation theory is valid. Stage (ii) is fragmentstion/hwdronization, where the initial partons are conbined by some prescription, and cotor-singlet hadrons are formed. In stago (iii), the short-lived hadronic restonances decay; it is their decay products and the other stable hadrons that reach stage (jv), experimental obeervation.

the first "direct" obecrvation of hard gluon raxliation. As an example of the obeerved jet structure, Figure 3.7 ghows events in SLD with 2 and 3 jets. As will be explained in the following sections, a number of properties of jets can be related to the underlying parton diatributions and hence can be used to test the predictions of perturbative QCD.

\subsubsection{Simulations of Fragmentation}

The caecade of quarks and gluon produced in a parton shower is far too complicated to lend itself to an analytical calculation of the exact final state with present-day 


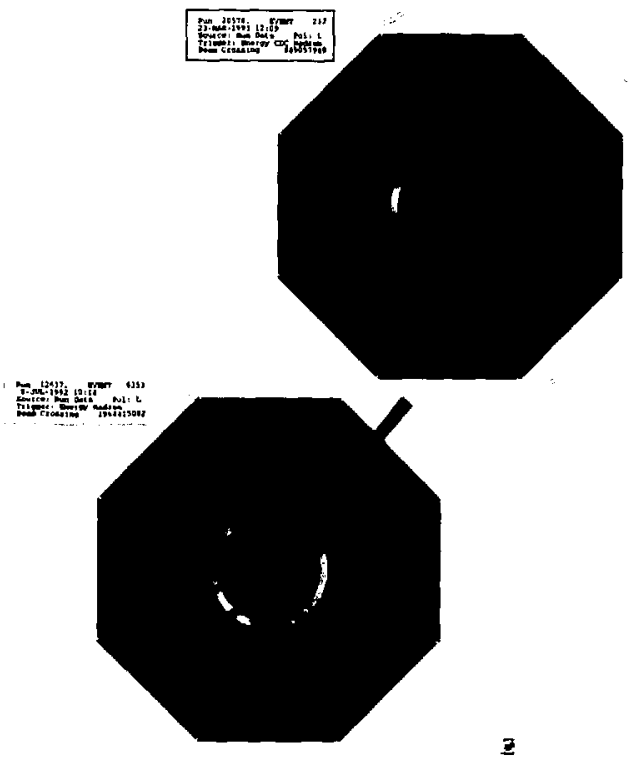

Figure 3.7: Views of 2- and 3-jet events in SLD. The curver! lincs originating from the center of the detector are cherged trucks; the irrczular rect wugular boxes which point back to the origin are encrgy deposits in the calorineter system. See Chapter 4 for a description of the various detector idrments. 
tecliniques'. Iustcad, various appecoeches haed on Monte Grilo simulation hxie been developed in an eflort to relate simple procewes contalniug susall numbern or partons to the properties of the hadronic final state[104]. They differ in the details of the shomering process, but all models contein a picture of the hedronic intersctions that boks something like Fig. 3.6.

To begin the shomring process, the initial state consisting of the four-monenta of the original pertons must be specified. Typically, this to done by either providing the calculated transition mutrix elements of the original distributions for some number of partons, known simply as the Matrix Element approech, or begind'sg with two partons and allowing then to shower based on splitting probabilitirs, known as the Parcou Shomer approecht

\section{Matrix Elements for Parton Production}

As dischssed in Section 3.1.2 the differential crows section for radiating a single gluon in events of the type $\mathrm{c}^{+} \mathrm{e}^{-} \rightarrow q \bar{q} \mathrm{~g}$ (see Figure 3.3 ) is given by

$$
\frac{d \sigma}{d x_{1} d x_{2}}=\sigma_{0} \frac{2}{3} \frac{\alpha_{0}}{\pi} \frac{x_{1}^{2}+x_{2}^{2}}{\left(1-x_{2}\right)\left(1-x_{2}\right)},
$$

where $\sigma_{0}$ is the lowest order Born crosis wection, and $x_{i}=2 E_{1} / E_{C M}$ for $i=1: \bar{q}, i=$ $2: g, i=3: g$.

Operationally, the writer of a Monte Cario simulation can avoid the infrared singularities of the matrix element by imposing bome sort of cut-off in the minimum inveriant mass between any tro of the partous, This corrsponds to modifying the the definition of what constitutes a 2- us. a 3-parton eveut depending on the energy of the radiated gluon.

As discussed above, some of the second- and third-order QCD matrix elements have also been calculated, yjelding the cross sections for 4 -jet $\left(e^{+} e^{-} \rightarrow\right.$ gipgg or $\left.e^{+} e^{-} \rightarrow q \bar{q} q^{\prime} \bar{q}^{\prime}\right)[87]$ and 5 -jet $\left(e^{+} e^{-} \rightarrow q \bar{q} g g g\right.$ or $\left.e^{+} e^{-r} \rightarrow q \bar{q} \sigma^{\prime} \sigma^{\prime} g\right)$ [88] wents. These

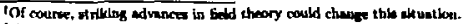

INoce that we have been weing the earm "perton sheref" to reler to the cancude of partone formed a tlo initial quarks fly apert from their production point. The Paton Shomer model of thin

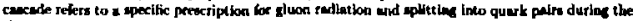
abowiting procesas.
} 
cass also be used to generate the hitinl-st ate perton distributious for conparison with experiment.

\section{The Parton Bhower Model}

Rather than attempt to calculnte the matrix elenepts fo,- all of the posible final sates to w high an order as poenible, one call we the Altarclif-Parlsi (LLA) formulation" and consicter the poesible branchings contained within QCD: $q \rightarrow \psi g, g \rightarrow q \bar{q}$, and $g \rightarrow g g$. Beginning with the imltial quark pair, the systetn can be evolved given the probabilitios that gluons are radiated, etc., as a functions of the parion energien and the energies of the radiated particles This proces would kook something like that shown in Figure 3.8. Typtcally, $a$, is allowed to "run" during the shower es the epergy of the partons decreases.

The shower process is inltiated with a salue of the energy of parton a end a suitubly choeen value of $t$, where $t$ describes the energy evolution of the system. The virtuality $t$ of the shower is allowed to run dowa until a brasching aecurs. Partome $b$ and $c$ arc then evolved following the same prescription, axd so on. Branching are allowed until the sivaleble $Q^{2}$ rceches some cut-oft valute $Q_{0}^{2}$, typically of $O(\Lambda)$. Note that the cutoff $\Lambda_{P S}$ depends on the actual definition of $Q^{2}$ choeen in implementing the parton shower and $\Lambda_{P S}$ is generally not equal to the $\Lambda$ introduced in renormalization.

Different inplementations of this model are distinguished by the definitions o $t, t$, sud $Q^{2}$. The implementation developed by the Lund group 105 ], for example, treats the shower procese in the center-of-muss frane, with $z$ being the cuergy fraction in that frame. The evolution paranetic $t$ is given by $t=\log \left(m^{2} / A^{2}\right)$, where $m$ in the mass of the parton. To account properly for the interference amons the many soft gicous that are emitted[106], an "angular ordering" can be imposed on the ahower such that successive gluou emisoions occur at smaler relative angles.

"Soe Section 3.1.4. 


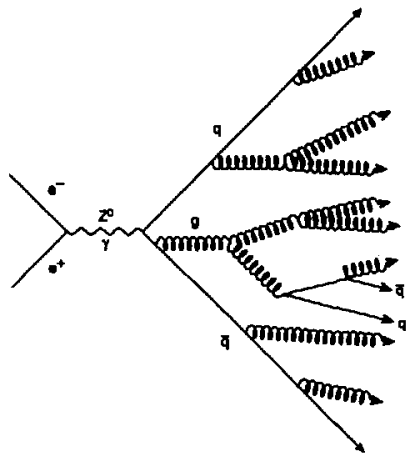

Figure 3.8: Parton Shower evolution, showing two primary quarks and n poosible set of their "Dranchings".

\section{Modele of Hedronization/Fragmentation}

Owee the initial parton diatributions are genernted from either the Matrix Element calculations or the cvolution of a Partan Shewer thcy must be "converted" into the cotor-singlet hadrons that can be obeerved by a pertich detector. The procese of this conversion is called fragmentution, $t$ and must be implemented by sorne model motivated by appropriate theoretical aseumptions, as the proceseces that form hadrons take place in the non-perturbative regine and are thereforc incukculable. There exist three major claskes of models for fraguentation:

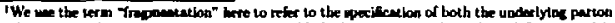

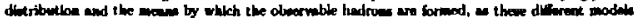
cal be didilegulated by their deacrigtion of both of these prycens.
} 


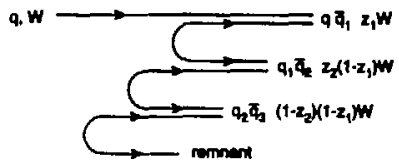

Figure 3.9: A schematic reprewentution of lindepeckest Fragmentation, showing the quark-autiquark pairs pulled from the vacuum and the energy fractions carried by each sucecuive hadron.

- The lindependent Fragmentation unde]

- The String Fragnientation model

- The Cluster Fragmenlation model.

Each of these will be descrilsed in some detsil in the following paragroph.

Independent Fragmentation: In this casc, cnch crurgetic parton in the event comesponding to a well-separated "jet ${ }^{n}$ is fragmented in Lolation. Sunll adjotments are made to the fragmented systemus to conserve ctwrgy and momentum axd to guarantee that quark flavor is conserved. The fregmentation proceses occurs in the following itcrative manner (sec Figure 3.9): an initial quark jet $q$ with energy $W$ becoulea paired into a luadron $q_{\bar{l}}$ carrying energy frection $W \cdot z_{1}$, lenving behind a remainder jet $q_{1}$ with energy $\left(1-z_{1}\right) W$. This jet in turn is split into another hedron, $q_{1} \overline{\phi_{h}}$, carrying energy $z_{2}\left(1-z_{1}\right) W$, leavitg abain a remnast jet $\psi_{2}$. The proces continues until the energy is too low to forn the lighteat hadron. The encrgy sharing between daughter products is given by a fragmentation function, often denoted $f(z)$, which is assuraed to be the barre at each step in the fragmentation process. The FieldFeynman model[107] as onc of the earlier vernions of this fragmentation acheme to be implemented in Monte Cerb calculations. 
This nimple approarh was made incresulingly complicated by the introduction of resonance production and decay, strangenew and baryon production, and mechanims to gcDerate an appropriste anoumt of tranverse inonentum friative to the initial quark direction. Varbous other independent fraguentation schemes of increas. ing comptexity have followed this initial formulation[108, 109]. The mon conmonly wed of these today is the ISAJET Monte Carlo|110).

String Framentation: The idea of using a string stretched between the uutgoing partons to represent the linear confinenent of QCD was frrt introduced in n Monte Carlo model by Artru and Mennesier[111]. Since then. the Lund group has been responsible for developing the model of string fragmentation as ne kiww it today. Their Monte Carlo sinuulation, JETSET 6.3[113], is used os the bagle for the results of this thesis.

In the string fragmentation model, the axis of the color flux tubet which is strutched bethen two jartons moving away from each olber is represented by a seniclassical massless relativistic string with no transverse degrees of [reedoun[114]. The otring is assumed to leave a uniform energy chensity por unit length. This gives both a lincarly rising confinernent potential and a coust ant probubility per unit length to split into new $q \bar{q}$ peirs that alow hadron formution. The splitting is done in such a way that energy, nomputum, and all internal çuantum numbers are conwerved. $A$ schematic view of the string splitting process for massless quarks is shown in Figure 3.10. Nawive quarks must act ually be produced sone dlatance apart so that the fiekd energy between them can lse transformed into maw und transverse moment ury. This can be uccusuplished by creating them at a point ask allowiug them to questunmechauscally "tunnel" ont to the allowed region. This tunneling process cecurs with a probablity proportional to [115]

$$
e^{-x+\frac{a}{T}}=e^{-x-\frac{1}{2}+e^{-a m^{2}}}
$$

where $\alpha$ is inversely proportionnl to the string constant. This formulation werves to generate tle transveres monventum spectrum of hadrons selative to the original quark

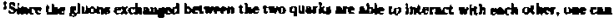

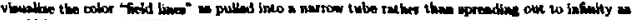
would be the cace in QED.
} 
(a)
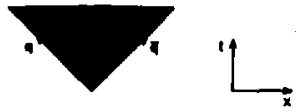

(b)

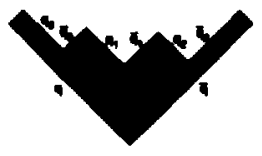

(c)

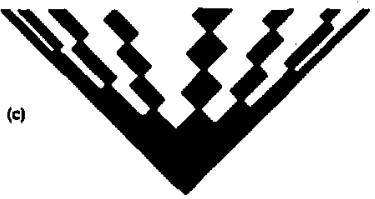

Figure 3. 10: A scthinatic representation of Striag Frukisuntation. In (a), the two primary çuarks fre shown ou they ituxт away from their consuon production point. The sladad region ts the physical extent of the inst-vastishiug color field. The fint three breakings of the atrive se showin in (b), with the guerk-enti-quark paire bebeled in the corder that they appeared. The further evolution of the shower is shown in (c).

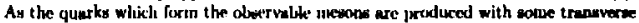
moneentunt relative to emch of her, they axillote about their censer of mon, which probluce the uepuestial rertanglex secil $a$ the mesons inowe towarda the top of the dingram. The uct und newon traject ory is the line drawi through the rectangle vertion. 


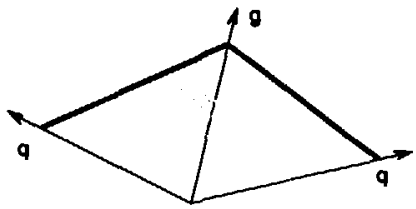

Figure 3.11: A qqg event in the string fragmentation scheme. The luenvy line is the string stretched between the glivon and the two quarks.

direction, and specifics by virtue of the relative quark mases the flavor composition of the $q$ of poirs ereated when the string is broken. Charm quarks, for example, are suppresed by a factor of $\sim 10^{-11}$ relative to the light $u$ and $d$ quariks and are esentially never produced in the string breakup.

These simple asi. mptions of the string picture lead to a number of interesting Featurs. Since the hedron production vertices are causally diseonaected, the timeordering of the production is Lorentz frmme dependent. Thim implies that all of the vertices must be treated jdentically, since no one branching is more primary than another, and leads to a an explicit form for the fragmentation function $f(z)^{5}$. The kinematks of string-breakup also lead to an ordering (on average) of hodrons in rapidity with respect to the primary quarks, as the slower hadrons are ptoduced earlicst.

The treatment of gluons is worth noting. The gluon is treated as $\mathrm{n}$ "kirk" in the string that is stretched between the two quarks (see Figure 3.11). This makes intuitive sense, siuce the gluon carries two color indices?. One consequetice of this fortnulation is that the multiplicity of hedrons will tend to be larger between the gluon jet and the two quark jets compared with tho region between the two quark

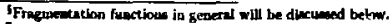

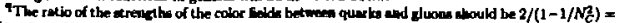
$9 / 4$, where $N_{C}$ is the mumber of colort. The vive a 2, then correponde to an ininile nurber of colon.
} 


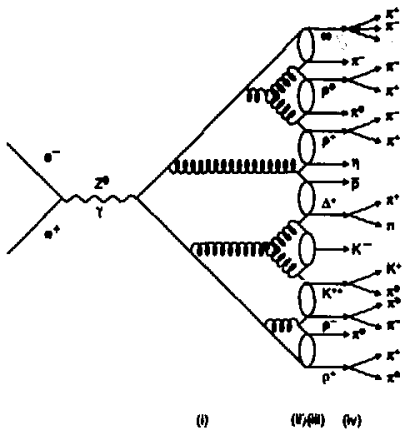

Figure 3.12: A lingusn of Cluster fregnentation.

jets. The first obwervation of this so-calked "string effect" was reported by the JADE collaboration in 1980[116], aud, after trore years of study, is now an establiehed part of the fragmentution! plenchisenology[117].

Cluster Pramentetion: In the Chuster Fragmeutation model, the Parton Shower evolution is given the prinnery role of producing the underiying structure of the final state hadrons by being allowed to evolve into many gluons a) 1 quarks. After brced splitting of the gluons into quarks, the quarks are combined into colorless "cluters", which are then decayed isotropically in their reat frames, typically wing sone aimple phase-spuce model. The asumption of the Cluster Fragmentation model. then, is that all of the properties of the hudronic final state should be determined by the perturbative QCD, rather than sone ad hoc model of non-perturbative fragnentation. A diagram of Cluster Fragmentation is shows in Figure 3.12. 
The fint widely successful model or this type was developed by Marchesini and Webbe[118], which incluchad a "culierent" parton shower approuch[119] containing prevloumly ignoked gluot interferesce effects[106]. The presest vetwion of this program is known HERWIG[120].

\section{Fraguentation Function:}

We int roduced abure the function $f(s)$ wilich goverss the shering of eaergy between ite dnughter hadrow aul the remuant jot that remains as the fregmentation process progreses. Here, we present witlout derication the two fraguentation functions used to guterate the Mouse Carlo events analyzed for this thenis. The Lund symonetric Iraginentation function[121] cns] le derived by requiring the left-right symmetries insolved in breaking a string starting at the quark ox the nutiquark side, and is given by

$$
f(z) \propto z^{-1}(1-z)^{\alpha} \mathrm{e}^{-6 \in 7 / 2} .
$$

Here, $a$ and $b$ are parameters which must be determined from experimental data. $A$ fit to the observed hadrun spectra in $e^{+} e^{-}$data gives typical values of $a=0.5$, $b=0.9 \mathrm{GeV}^{-2}[104]$.

For ments contuining heavy qunrks, experimental results indirated that a "harder" Iraginentation function nas mectessery: The so-called Peterson form][122] has become the stundard paramatrization:

$$
f(z) \times \frac{1}{=\left(1-\frac{1}{z}-\frac{i}{1-z}\right)} .
$$

Typical values for $\varepsilon$ are $\epsilon_{c}=0.06$ and $c_{b}=0.006[123]$. These valuts and the Peterson fragnentation function were used to generate the heavy quark Monte Carlo events for this thesis.

The remainder of this chapter is divided somewhat arbit rarily into mections on teclwigues for measuring ithe st rong conpling $a$, and wet hods for testing the properties of QCD outlited above. The division is arbitrary in the sense that measuring $a_{\text {, }}$ with different techniques anpl conpuring the resulta sctually provides a test of the calculational methods used to derive the theoretical predictions wed to nake the measurement[124]. 


\subsection{Measuremente of $\alpha_{s}$}

To begin the discusaion of techniques for neevuring the atrong couplims, we reand the reader of the matrix elesient for $\mathrm{e}^{+} \mathrm{e}^{-} \rightarrow$ qqg exents shown in Eq. 3.94

$$
\frac{d \sigma}{d x_{1} d x_{1}}=\sigma_{0} \frac{2}{3} \frac{a_{1}}{\pi} \frac{x_{1}^{2}+x_{2}^{3}}{\left(1-x_{1}\right)\left(1-x_{2}\right)} \text {. }
$$

Au night be expected, the rate for the redintion of a gluon is indeed fropurtional to the coupling $a_{0}$. So, a mensurement of the rate of nitgle gliton cmiosion whould, $\alpha$ this simplistic fevel, allow the derivation of a value for the strong coupling. From the

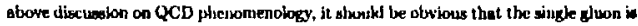
not observable, and that instesd the find state will contain some number of hadronic jets. So, meawuring the rate of single gluon enimikn corresponds experimenally to measuring the rate of expets that appear to heve three jets. A diacuesion of whot thiactually sneans will follow. We present this first as it is not only the mom inculeive method, it is the one uscd in thiy thesis.

\subsubsection{Jet Rates}

As was mentioned in the discussion following Eq. 3.94, the differential croes rection diverges as $x_{1}$ or $x_{2}$, the fraction of the beam energy sarried by the outgoing quarke, approaches unity. This cortesponds to the instance when soft gltwons are emittod aloes the quark direction but are still counted as ixhependent partons. When combined with the lowest order propagalor and vertex tortections, floc result actually is finite, bot this belaviour prints to a connont resolution to the problem of infrared divergences. To sidestep tlue collitiear divergeaces, a mww cut-off can be adderl to the theory, such that any two partong whose combined invurimit mast is less then sone parameter $y_{\text {min }}$ are congideral as an unresolvable siogle purton. This makes particular ane when one is dewling with a final hadronic stute containing jets rather than individual

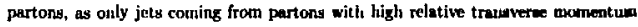
are distinguishable txperimentally. The firat tweswrement of $a$, wing the rate of "resolvable" 3-jet eventw was published by the PLUTO collaboration in 1960|125]. 
All of the jet alorithms currently in wase are based on a procedure of iterative cluster-finding. Firs, the user specifies the manner in which the invalant "mass" yy should be calculated. An axample, from the algorithm developed by the JADE collaboration:226], is

$$
H_{j}=\frac{2 E_{i} E_{j}\left(1-\cos \theta_{i j}\right)}{s},
$$

where $E_{i}$ in the avergy of the $i^{\text {th }}$ particle, and $\theta_{i j}$ is the angle betwett particles $i$ and $j$. The algoritum then considers all of the particles in the event and continice the two with the lowest $y$ value into a single peetodo-particle following some prexcription, like $p^{\#}=p_{i}^{H}+p_{j}^{\prime}$. The procese is repeated using the remaining particles until all of the (pseudo)- particles have an Invariant mass greater than $y_{\text {min }}$ (also reforrod to a $y_{\text {cou }}$ ) when combinations with al) otber clusters are tried. The number of clusters remaining is then the number of jets in the event.

Initially, this manper of measuriog $\alpha$, was limited by the omiseion of harge nextto-iending terms from the thenretical calculations. Results up to second order in $a$, have now been inciuded, aud attempts bave been made to raduce the dependence on ine renormalization parameter $\mu$ as well on the uncalculated higher-order terms[127]. The general form of the predictions for the 3 -jet rate $\sigma_{3 j \mathrm{jet}} / \sigma_{0}$ is $[12 \mathrm{~s}, 12 \mathrm{w}]$

$$
\frac{\sigma_{3 j e t}\left(y_{\text {out }}\right)}{\sigma_{v}}=\frac{a_{s}(\mu)}{2 \pi} A\left(y_{\text {rut }}\right)+\left(\frac{\alpha_{s}(\mu)}{2 \pi}\right)^{2}\left\{B\left(y_{\text {ow }}\right)+A\left(y_{\text {ow }}\right) 2 \pi b_{0} l_{0} f\right] .
$$

Here, $f=\mu^{2} / s$, and $b_{0}=\left(33-2 n_{\rho}\right) /(12 \pi)$, where $n_{\rho}$ is the number of active flavors $\left(n_{j}=5\right.$ at $\left.\sqrt{0}=M_{z}\right)$. The terms proportional to $B$ arise in part from 4-jet events where two ol the jets were unreso.valuk. The 4 jet rate $a_{4 j+1} / a_{0}$ hes only been calculated to lonest order $\left[8 \tau_{1}\right.$, and has the expected fonm:

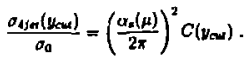

In general, the coefirients $A\left(y_{\text {owe }}\right)$ and $C\left(y_{\text {ow }}\right)$ are the same for all algorithms, since there ahoind be no dependerwe of the lowest order nesult on the choice of some specific jet-fiuding algorithw. It should be noted that these results are a comparinon of the second-order fet production rates with the unul Born croses action $\sigma_{D}$, where

$$
\sigma_{0}=\frac{4 \pi 0_{0}^{2}}{3} N_{C} \sum_{l} Q_{I}^{2}
$$


The tree-level croes section ls aleo modilied by QCD correction, which have actually been calculated to third order in $a$, for studies of the $Z^{7}$ hadronic width[120]. The: secoud-order result in the $\mathrm{MS}$ icbme $\left(\Lambda \equiv \Lambda_{\mathrm{KB}}\right) \mathrm{m}(130)$

$$
\frac{1}{a_{0}} \sigma_{\text {sox }}=1+\frac{a_{s}\left(Q^{2}\right)}{\pi}+\left(\frac{a_{s}\left(Q^{2}\right)}{\pi}\right)^{2}\left(1.900-0.115 n_{j}\right),
$$

whe re the corresponling second-uxcher value of o, we giveu above in Eq. 3.118. An exporiment messuring jet production rater necenurily nucuures $\sigma_{\text {njet }} / \sigma_{\text {ood }}$, so these corrections should be taken into acownat when ureasuremente of $\alpha$, are derived from these quantities.

\subsubsection{Event Shape Variables and Particle Corrolationa}

Various other properties of hadronic evends in $e^{+} e^{-}$ennihilation evemes can sho be cakulated in pert urbative QCD. Theuretical predictikn similar in form to Eq. 3.130 exist for quantities related to various dish ributions of perticle monenta about the jet (or some other) axis and the flow of hedrun in the everix; measurements of $a$, can then be derived from the observed dintributions. Rather then liat and deseribe all of the other techniques for masuring $a$, bere, we refer the reader to Ref. [128], which presents an overview of hadronic olwerialdea in $c^{+} c^{-}$annihilation at the $Z^{*}$ pole. $A$ recent paper from SLD [131] couteins u comprelensise set of mewurements of $a_{\text {a }}$ using these techniques.

\subsection{Tests of QCD}

The emergence of Quantum Chronodynamics as a visble theory of hadronic interactions (marked by the discovery of myraptotlc freedorn) coincided alnoet exectly in time with the final piece of evidence that the quark unokel of hedronic structure was correct (the discovery of the chermonium states in 1974[132]). The predictions of QCD were now gubject to experimental scrutiny, an new experimental results were 
compared to all of the different pasible models of the strong force in order to determine the "correct" theory of the strong forte".

In additlon to the r rucial suceses in dexcribing the phenomenotoxy of deep inelestic scattering, the fin: observation of jets in $e^{+} e^{-}$annililation[103] provided anothy early triumph of the theory, ws jets are esentially a result of quark confinement. As mentioned ubove, the observation of 3-jet events at PETRA|81] gave the firgt direct evidence for the gluor, though neutral, chargelese hadronic constituents had been required earlier to make the quark-parton model agrce with deep inelast ic acattering data[134]. It was also shown quickly from the angular distribution of the gluon jets that the radiated parti le was consistent with having spin 1[135]. Another important

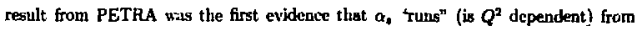
studies of jet-production rates at different center-of-mass nnergies[136]. Dirict cvidence for gluon sclf-coupling whos fouts in the orientation of the jets in 4-jet events found at TRISTAN[137]. Eien as these basic terets of QCD liave been verified, efforts continue in testing the theory on many fronts.

\subsubsection{Flavor Independence of $\alpha_{s}$}

One active aren of investigation is tests of the flusor independence of the strong coupling. Iu priuciple, the QCD Lagrangian, iustcad of looking like Eg. 3.91, could have a swn over flavor:

$$
\mathcal{L}=\sum_{j}^{\eta_{f}}\left[\hat{\gamma}_{j}\left(i \gamma^{\mu} \partial_{\mu}-m_{j}\right) q_{j}-g_{\alpha}^{j}\left(\bar{q}_{j} \gamma^{\mu} \mathrm{T}_{a} q_{j}\right) G_{\mu}^{a}\right]-\frac{1}{4} n_{f} C_{\mu \nu}^{\prime} C_{a}^{\mu \nu} .
$$

However, this formulatron violstes the Wurd-Takahasli identity[138], which, by virtue of color conservation, II guires that the coupling in the three-pluon vertex lye the sause as that in the $g q \bar{\eta}$ vertex. A brief discussion of the implications of the Ward-Takahashi identity and its importance is in order.

\footnotetext{
An early indlewtion that color for some odwer wew quaneurn nutuber allowitg three itrkependent

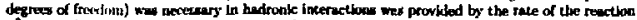
$x^{\circ} \rightarrow 77$. A calculatton of this rate proved to be incompatible whb experimentel obuervitions unkew each "quark" that contribut od to the final amplikude was counted three timsed133]. However, this ras realiand before the full applicabitity of QCD becuse eppunat.
} 


\section{Tho Ward-Talaluent Identity}

To introduce the ideutity, we return to the more familiar environs of QED. Gurs and Lorentz invariance of the electronagiatic field $A_{\mu}$ requires that free pholons heve only tranoverse polarizution states, i.c., that the cundition

$$
k_{\mu} e^{\prime \prime}(k)=0
$$

can be reduced to

$$
\vec{k} \cdot \vec{c}(k)=0,
$$

where $k$ is the phnton monent $u m$, and $e(k)$ is the ploton polarization vector. This can be seen in the foilowing menner. The free photon field has the $t_{i}, A_{\mu}=c(k)_{\mu} e^{-i t-x}$,

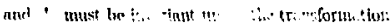

$$
A_{\mu} \rightarrow A_{\mu}^{\prime}=A_{\mu}+\partial_{\mu} X
$$

where $X$ satiafics $\partial^{2} \chi=0$ to insure that $\partial^{3} A_{\mu}=0$. We are free, then, to cboome a gauge paranuicr

$$
X=i a e^{-i k \cdot s}
$$

where $a$ is some constant. Under the conditions of $\mathrm{Eq}_{\mathbf{q}}$ 3.137, the physics numb be unchanged ff the polarization vector $\epsilon_{\mu}$ is replaced by

$$
\epsilon_{\mu} \rightarrow \epsilon_{\mu}=\epsilon_{\mu}+a k_{\mu} .
$$

This implies that two polurization vectors which differ by a multiple of $k_{\mu}$ describe the same photon. This degree of freedom implies that there are only two independent polarization vectors to deras the phototi; we can require the time component $c^{0}$ to wuish identically, $\epsilon^{0} \equiv \mathbf{0}$. Then, stupiring the field $A_{\mu}$ to sacisly Maxwell's equations ior a fiee field gives us the further condition that the polarization must be perpendicular to $k$, which results in the condition of Eq. 3.136.

We can now exemine some of the consequences of the relationabipe derived ubove from geuge invariance. Given uny anuplitude $M_{1}$ including an exterial photon of 
polarization $\epsilon_{\mu}(k)$, the following relation mut holdt:

$$
k_{\mu} \cdot M_{i}^{\mu}=0 \text {. }
$$

We have used the abbecript I bere to denote the amplitude lacluding the external photon. Although we have not proven it here, this rrsult is true to all orders of perturbation theory. If we define the amplitude $M_{0}$ ss the sane set of disgrams without the photon, then we obtain $\mathcal{M}_{1}$ by summing the diagrams $\mathcal{M}_{0}$ with the additional photon inserteri at every possible point. For an smplitude $M_{1}$ with $n$ incoming and $n$ outgoing fersion lines (and an arbitrary number of external photons), the gemeral form of the Ward-Taknhashi identity is glven byt

$$
k_{m} M_{i}^{*}=q_{s} \sum_{i}\left[M_{0}\left(p_{1} \cdots p_{n} i q_{1} \cdots\left(q_{i}+k\right) \cdots\right)-M_{0}\left(p_{1} \cdots\left(p_{i}+k\right) \cdots ; q_{1} \cdots q_{n}\right)\right] .
$$

Here, $q_{f}$ is the ferrion charge, the $p_{t}(l=1, n)$ are the momenta of the incoming fermions, and the $q$ are the moments of the outgoing fermions. In the case that $M_{l}$ is an sunplitude for sone physical process, the two unplitudes $M_{0}$ do not contribute, and the original form of the identity (Eq. 3.142) is recowered.

The singitest example is shown in Figure 3.13a, where we have a froe quark propegating the findernental Drocess $\mathcal{M}_{\mathbf{0}}$. If we add un external photon, this gives the digram on the right. hand side, which we denote $M_{1}$. In this case, there is only one diegran in the sum ove ineertion points, since tbetc is only one place we cars. attach the ploton to the quark line. From Equation 3.143, we find the situation abow $v$ in Fig. 3.13b. The components of them graphs are simple, as they are given.

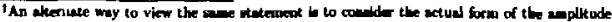

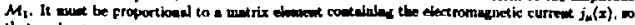
thet we teve

$$
M_{\mu}(p) \propto \int d x e^{-s p}\left(J\left|j_{\mu}(x)\right| i\right) .
$$

Slace $\partial_{\mu} j^{\mu}=U$ by current nas rvalion,

$$
k_{\mu} M^{\mu} \propto \int d x e^{-\omega}\left(J\left|D_{\mu} j^{\mu}(x)\right| j\right)=0 .
$$

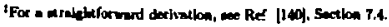




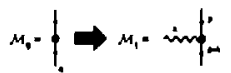

山1

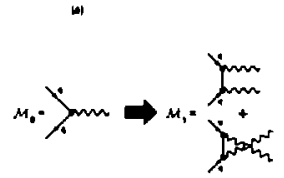

(a)

$$
\mu_{1}^{*}=n_{*} \times(f)-\left\{\begin{array}{l}
m \\
m
\end{array}\right)
$$

a

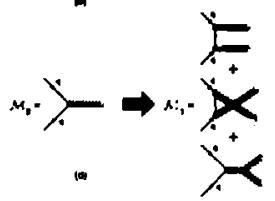

Figure 3.13: An illustration of the amplitudes involued in a discuesion of the Werd. Taloahashi ldentity. In each case, the diagrams labelod $\mathcal{M}_{0}$ ropresent the minplitude for some process without the addition of an exterad photon or gluon. The diacrans labeled $M_{1}$ make up the aniplitude after the external photon or gluon hing been added. In (b), a clisgramatic representation of the identity is given for the simpled cawe. Soe the text for a description.

by fermion propagators and the vertex function. Let us discuss the ranifications of the Ward-Takahush identity on the renortnalized nuas and charge in QED by uino the renormalized fermion propugator and vertex functions. Then, we haves

$$
S(p)=\frac{i}{-m-\Sigma(p)}
$$

for the fermion propengator. Here, $\Sigma(p)$ is the sun of all (infinite) one-particle irreducible virtual corrections to the fermion lise, and $m$ is the fermion mave. The multiplicative senormalization coeflicien $Z_{2}^{9}$ is defined by

$$
Z_{2}^{-1}=1-\left.\frac{d \Sigma}{d \phi}\right|_{j=m}
$$
7.

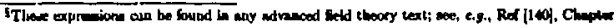

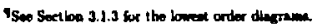


- and is the residuc of the propragator pole

$$
S(z) \sim \frac{i Z_{2}}{1-m}
$$

at the physical nunse in. The vertex function is denoted thy the symbol $\Gamma^{\mu}\{p+k, p)$, whach repraxents the sun of all of the corrections to the Lure vertex. The renoriun]ization constant $Z_{1}$ is given by

$$
\Gamma^{\mu}(p=0)=2{ }^{-1} \gamma^{\mu} .
$$

If we set $p$ near the mass shell, nul expausion of E(1. 3.143 to lowest order in $k$ gives

$$
-i Z_{1}^{-1} \not=-i Z_{2}^{-1} \not
$$

or

$$
Z_{1}=z_{2} \text {, }
$$

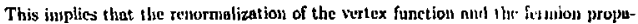
gator aue not independent. If ur rmeill froll uur discussion of the renormalization of the atrong mojting, Eq. 3.98 , that the renonuslization of the charge is given by

$$
e=\frac{Z_{2} \sqrt{Z_{3}}}{Z_{1}} e_{0} .
$$

we cun casily see that the renormalization of the charge is given only by the contribution from the vacuum polarization of the plioton, represented by the factor $Z_{3}$. That is, the clectrite clarge renornadization must be universu, $n$ result which is valid to all orders in perturbution theory. Note that different fermions are allowed to lave differcut electric churges; the ratio of the charges can be expresed in terms of a universal constant $c$.

The next most complicated case is shown in Fig. 3.13b, in which we add an extra photon to a $49 \gamma$ verlex to obtails quark Conspton scattering. In this cabe, the sune cancellation must occur. This can be compared to the diugrame of Fig. 3.13k, whitis shos' the same process in QCD, with photons replaced by gluons. Although the derisation of the Ward-Tularlasshi identity is more difficult in Youg-Milis theories[139], the qunlitative result is the saute: $\mathrm{k}_{\mu} \mathcal{M}_{1}^{\mu}=0$, where $\mathrm{k}_{\mu}^{\mathrm{g}}$ is the momentum of the 
external gluon. in QCD, the presence of the three-gluon vertex here demands that the coupling between gluoss must be the same as that letween gluots and quarke, or elwe QCD censes to be a unitary, renorinulizable theory. Thus, the stroug "charge" or the maguitude of the serong coupling muat be the sanke for all quari, ilawort. Again, this numat be true to al] orders in perturbation theory. It [s through this meclianisun thut unsdifications to the legrarjgjan like that of Eq. 3.134 are forbidden.

\section{Other Menns of Iwtroducing Flavor Dependence}

Undaunted by field-thenretical considerations, we can still entertain tic powitsilities of different strong coupling strengths for each of the quark fluwors, since, if the experiment nlly neasursol couplings were to be unequinl, new pliysies must be present. Vartly different couplings among the light quarks can be dismisterl by the consideration of the rpectrum of hadrons seen is $c^{+} c^{-}$interactions, wa a large discrepancy between $\alpha_{\text {* }}^{*}$ is $!$ ' would violute the apparent $S U(3)$ llavor und iswopil symmetries observed in nuture.

Nor" possible, perhurys, is a difference in the couplings of the light and beavy quarks, through some tnechanism involving powets of the quark masses which would supjsrss the effect for the light quarks. If, for example, a quark had some son of cluromo-magnetic noment, its effective coupling to the strong interaction would be different then quarks laxking this extra vertex currection. This sort of effect has been propoued in the context of studies of the top quark (141), but it is equally applkable herc. In this sort of theory, the sernisl term of the QCD lagrangian would be replaced by

$$
\mathcal{L}=g, \bar{q}_{j} \Gamma_{\mathrm{u}}\left(\gamma^{\mu}+i-\frac{F_{2}\left(k^{2}\right)}{2 m_{j}} \sigma_{\mu \nu} \mu^{\mu}\right) \boldsymbol{q}_{j} G_{\mu}^{a} .
$$

Here, $k$ is the outgoing ghon monrentum from the $q_{j}$, g,g vertex, and $F_{2}\left(k^{2}\right)$ is a form fartor descriling thus strengtlis of the anomuluus coupling. This term does not violate the Ward-Takalushit idmetity in QCD. atul thus is permissible. This is usually puxamotrized by a coustunt such as $x$, where $F_{2}\left(k^{2}=0\right) \equiv k$. Performing tho 
calculation of the 3-jet ratc with these terms in the lagrangian yiclds

$$
\frac{\sigma_{\text {et }}}{\sigma_{*}} \simeq \frac{\alpha_{1}}{\pi} \frac{n^{2} s}{18 m_{1}^{2}} \frac{v^{2}+1.25 a^{2}}{v^{3}+a^{2}}
$$

where a (v) is the axdal (vector) coupling of the quarks in the electrowesk interaction. If $k$ is non-zero for some quark, the measured value of $\alpha$, for that quark will be larger than those with $k=0$. New couplings of this type do not arise in the "uwual" extensious of the standard model, so the observation of en enonalous strong coupling for a specific quark flavor would he a sigll of truly "new" physics.

Tests of the flator indepentidtice of QCD have been uade possible by the accumulation of $r$ iulively large data sets contmining five quark fluvors at PETRA, TRISTAN, and at LEP/SLC. To perform a teat of tjis sort, an eveut sample containing predominantly the quark favor of interest must be obtained and an analysis performed on this sampic to yield a value of the strong coupling for that quark favor. Methods of flavor tagging linve bees devcloped which allow the isolation of events containing printary quarks of r particular flavor. The mepration of heavy ( $b$ and $c$ ) and light (uds) quark cveits is the inost straightionesid dise to the long lifetimts, hard fragmentation functions, and large masses of the hudrons that contain licavy quarks. A curonological discussion of the previous tests of Havor independence will serwe both to introduce the various flavor-tagging tecbriques und to summarize the existing results.

The TASSO Collaloration carricd out the first tests of the flavor independence of $\alpha$, at PETRA. Evouts containing charm quarky were identified by exclusive reconstruction of $D^{\cdot}-\pi D^{0}$ meson decsys and the EEC distribution was used for the measurements of the strong coupling. They obtaived $[142] \alpha_{g}^{c} / a_{a}^{e l l}=0.91 \pm 0.38 \pm 0.15$. It ls worth noting that this partieular technique of selecting cherm decaya nectesarily roquites the charm moson to be carrying a large fraction of the energy svailable to it, which limits the acceptance for events with very hard gluons. Also, cluarm events containing the appropriate $D^{*} \rightarrow \pi D^{0}$ cascedes only make up a small fraction ( $\sim 7 \%$ ) of the total and the exclusive decay tnodey used to reconstruct the $D^{0}$ have small bronching fractions. These factors lead to an undesirably smoll efficiency for tagging charm events and hence to an undesirably large statisticul error. Events containing

\footnotetext{
Isee the other half of thlo thesin for a dhcumbon of there lechniquen.
} 
$b$ cuarks were tagked by searching for the presence of decay vertices well- separated from the beam interaction point. They obtained[143] $a_{*}^{+} / a_{*}^{-1}=1.17 \pm 0.50 \pm 0.28$ from the EEC distribution. Using lifetinc information to tag heavy querks is made ensier due to the loug typical Hight distances of the heavy tneachs and the lerge avalable enersy in the meson decay. These factors result in sufficionit numbers of charged tracks erstenating fron the tresuen decay polnt with relacively large tranoverse momentun relutive to the meson flight direction to make mearcluing for decay vertices, for exumplu, an efficient process. This method of tagging is ulso unch less dependent on the kinematic propertics of the event, as the vertex flight diatance resolution is typically much smoller than the mean meson decay length".

The ern of experimentation at tle $Z^{0}$ pole with $\mathrm{e}^{+} \mathrm{e}^{-}$colliders has allowed tests of the fluvor independence of QCD with unprecedentel jrexision. All of the eariy results from the LEP experiments were prodiced without the present set of precinion microvertex detectors that allow tle type of $b$ tagging dune with TASSO. Instead, kineniatic tags were used to sepurate events of different flavors. The most common is to use identified leptons will, lifge momentum and large trajkscrse nomentum relative to the neareat jet uxis as a tas of $b$ or $c$ quarks" Depending on the cuts placed on the lepton momente, this netliod could also bias the event sumpte away from those evettes containing hard givon sadiation. The lepton tog spprosech was followed by the L3 and DELPHI collaborations, who found $\alpha, \alpha$, were $=1.00 \pm 0.05 \pm 0.06$

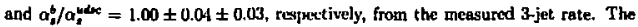
OPAL colinboration[145] performad the first truly corpprehensive stuxly of the flavor independence of $a$. They used high $p_{1}$ keptons for $a b$ tos, exclusively reconatrueted $D$ mesons for the charm tag, fast $K_{b}^{* 0}$ for a strange tag $\left(x_{K}=2 E_{K} / E_{G N}>0.4\right)$, and fast pions, protony, and kaous $\left(0.7<x_{n k}<1.07\right)^{\text {tt }}$ as a taf of light (uds) flevors. The 3-jet rate is measured, and a graud unfolling is done to oblain the ratios of

\footnotetext{
"Althouch, thin wes not the cae for the TASSO vertex detectox, which explairs thetr larte statiotical error and pclatively and anding eficieticy.

t'See Section 2.8.1.

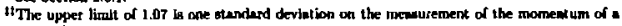
partleb with $I=1$.
} 
couplings:

$$
\begin{aligned}
& n_{*}^{6} / a_{*}^{\text {ant }}=1.021 \pm 0.013 \pm 0.023, \\
& o_{j}^{p} / \alpha^{d / t}=0.912 \pm 0.067 \pm 0.061 \text {, } \\
& a_{a}^{*} / \alpha_{s}^{d t}=1.141 \pm 0.043 \pm 0.142 \text {, } \\
& 0: / o_{*}^{* 4}=0.033 \pm 0.087 \pm 0.175 \text {, } \\
& \alpha_{\mathrm{N}}^{\mathrm{N}} / \alpha_{\mathrm{s}}^{\text {ell }}=0.951 \pm 0.103 \pm 0.182 \text {. }
\end{aligned}
$$

The large systematic crrors on the light quark couplings are due to the uncertainties in hwsing a tog on tbe identity of the fastest particle in an event, as this is uncharted territory for many of the Nonte Carlo models of hadron production. The large statis-

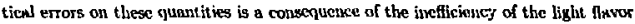
tags. A recent paper by OPAL has extended the repertoire of tecluniques used in the ineusurement of $\alpha$, for the flavor tagged samples[146]. They use a detached vertex $b$ tag similar to the onc developed at TASSO to obtain a pure sumple of $b$ events. A large number of mentureumts of $\alpha$, are then performed on this $b$ sample, and the results are averaged to state a single value: $a_{\$} / \alpha_{3}^{a l l}=0.994 \pm 0.005_{-0.020}^{+0.010}$. This is by far the noost stringent test of the flavor independence of $\alpha_{\text {p for }} b$ quarks.

One feature of oll of these analysos is that a ratio of couplings like $\alpha_{j} / \alpha_{4}^{\prime \prime}$ is meusured. This has the balizntage that it reduces the effects of a number of errors that can plague the determination of $\alpha_{\mathbf{n}}$. For example, if concetions due to detector resolution or acceptance are extially the same for each flavor, the uncertainties on the ratio due to these corrections are smallec. Uncestsinties due to the dioice of reporiualization scale $\mu$ should also motly cancel, since the evolution of the parton showers are identical in all events up to the uflects of quark masses.

In all but the OPAL analysis involving five flavors, asonumptions need to be niade about the relatise strengths of the stroug coupling for the other quark flavors, at no sample of events is $100 \%$ pure, and the background being subtricted has sonic 3-jet

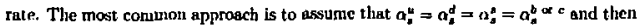
proceed with the backgroutud subtraction. 


\section{Methodis Cbowen for This Analyois}

In an attempt to obtuin the best poesible statintical precision for all flavory, whe choeen to pursuc an "inclusive" aralysis to teat the flavor independence of the atrong interaction. The method is inclusive in that all hedronic events which pass the wkection suts (Clinpter G) are ued. We wo the precision microvertex betectoc to separate the fiavors baved on the charged multiplkity of secondary decays*: there are no decays of henvy secondary particles in uds events, and the average number of secondary tracks in $c$ and $b$ quark cveits differs by aimost 3 tracks. The tas b based on truck counting; reconstructed decay vertices are not required, th this reaults in tags of lower efficiency. Using the nuenber of trucks that are not conaistent with originating fron the precisely determined powition of the intcraction point, we can obtain highly enriched sanjples of events containing primary uds and $b$ quarks, with . somewhat enriched semple of $c$ quurk events in the remainder. This tayging process is relatively insensitive to the underlying event kinenutica, and results in event tagsing efficiencies that are quite Jigh. We note in pasing that it is the tiny, stable beam spot of the SLC that allows us to tag the uds sample. We do not need to obtain the position of the interaction point for crery event, a procedure which could introduce large systematic effects for a tag of this sort.

We also perform a jet rutes unalysis on cach of the tagsed souples, and the

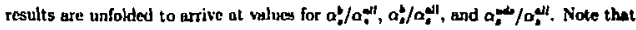
by using all events and unfolding to artive at values for $a_{a}^{3} / a_{s}^{\text {all }}$ we ouly make the

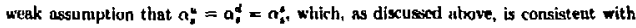
the approximate isospin and $S U(3)$ Havor symmetry observed in badronic structure.

To determine $a_{a}$ ratiog from the jet rates measurements, we use six of the commonly used jet-fivuling algorit hnas in order to coshpare the effects of the uncalculated higher-order terms in the the $3-$ and 4-jet croses yections (Chapter 8). Our results will be discussed and compared with those nemtioted above in Clispter 12.

"The method of bavar teg will be explaiged in great detail in Chapter 7. 


\section{Chapter 4}

\section{Experimental Apparatus: the SLC and SLD}

This chapter presents a description of the unique facility that exists at SLAC for the study of $Z^{\mathrm{a}}$ bowon physics. Added detail will be included in the sections that are relevant to the analyses presented here.

\subsection{The SLAC Linear Collider}

The S!.AC Linear Collider (SLC) is the world's first lizear collider, a an accelerator concept which gaitued prominence[147] in the 1970's iss perhaps the only econarnically feasible way of pustring $\mathrm{e}^{+} \mathrm{e}^{-}$colliders to the high energy frontjer. The prototypical linear collider consists of two linesr sccelerators (linscs) producing high-intensity beams which impinge on a common interaction point (IP) which is surroundexl by the particle detcetor that will record the interactions produced in the collinions. In their inimitable SLAC faskion, the designers of the SLC, when faced by budgetary and spatibl constraints, produced a linear collider folded back on itself, as shown in Figure 4.1. Both the $e^{+}$and $e^{-}$benms are accelerated by the linac and then the beams are split, bent $00^{\circ}$, and forced to collide bend-on. One of the salient features of a linear collider is that the beam bunches cannot be in imediately re-used 


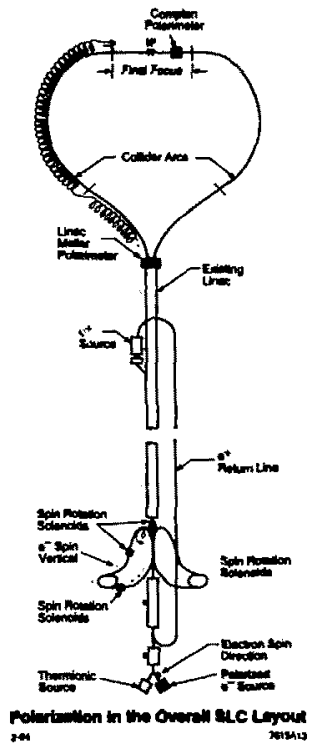

Figure 4.1: The Polarized SLC, showing the overall layout of the scorletator complex. The orientation of the electron epins is gives by the arrows along the path of the beam. SLD sits st the IP. 
- for collisions as they are in a storage ring collider due to the violence of the bearnbeam interaction. Some schemes exist for recirculation of the beams[148], but here the speat bunches are sboorbed in a beam dump after each collision. Another "feature" is the low repetition rate of the acceleretor, which is limited by power constraints as will as the maximum operational frequency of the pulsed-magnet "kickers" which perform nexrly instentaneous steering of the beams along the desired orbits. In order to obtain a sufficiently high interaction rate in the beam collisions, it is necesary to focus the porticke treams to incredibly tiny crost-sectional areas, which is accompliehed by a complex optical system known as the "Final Focus" inmediately prexoding the interaction point. Some of these features will be discussed in more detail in the following seetions.

\subsubsection{The Polarined Electron Source}

The SLC is unique in its ability to accelerate, transport, and collide a longitudinally polarized bean of electrons. This is unado possible by the introduction of Galljum-Arsenide [GuAs) as the active matcrial[140] in a photocathode- besed electron gun[150]. A circularly polarized laser is used to electively excite transitions jnto longitudinally-polarized states in the conducrion bund. An energy state transition diegram is shown in Figure 4.2. In 1992, a bulk GAs cathode was used which had n theoretical maximum polarization of $50 \%$. Thu aturage polarization measured[151] wax 22\%. In the 1993 run, a new strained-lattice cathode[152] using GaAs grown on a GuAsP (Gallium-Anenide-Phosphide) substrate why enjploycd, yiciding an avcrage polarization of $\sim 65 \%$ at the source (see Figure 4.2). A new cathode with a thinner GaAs layer is currently yielding average polerizations of $\sim 80 \%$.

\subsubsection{Beam Transport}

A single accelerator cycle begins with the production at the source of two bunches of electrons cach contaiuing approximately $5 \times 10^{10}$ particles. These are captured ly the accelcrator, and accelerated up to an energy of $1.19 \mathrm{GeV}$ at which time they are slored in the North damping ring. The damping ring reduces the size of the 

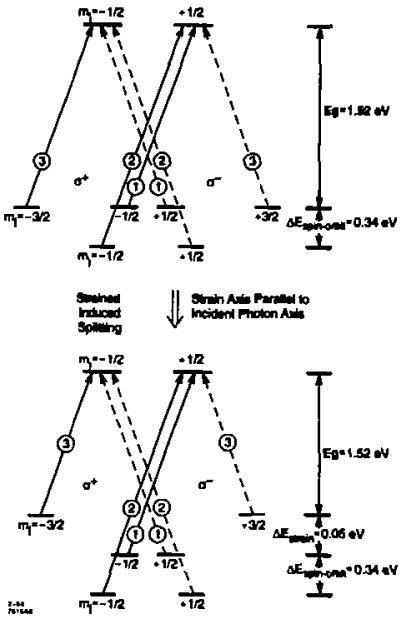

Figurc 4.2: The energy state diagram for bulk GaAs (top) and the changes it undergoes when the lattice is strained (bottom). The relative gizes of the matrix elements governing the interatate transitions are given in the circles. For excitation of electrons to the $m_{j}= \pm 1 / 2$ statc, the theoretical maximum polarization is $50 \%$ for bulk GaAs (top). For the strained lattice, the degeneracy between the $\left|m_{j}\right|=3 / 2$ and the $\left|m_{j}\right|=1 / 2$ valence states is broken, allowing the potential for $100 \%$ polarization. 
beam phaee-space through the emlasion of synclurotron rediation and the application of radiofrequency power. After a large number of turns, they are extructed from the ring and folkw a positron bunch from the South demping ring down the linac. Approximately two-thinds of the way down the linec, the eecood electron bunch is diverted osto a target to produce poeltrons, which are then captured and sent buck to the South demping ring to awat the next accelerator cycle. After reaching their maximum enersy of $46.7 \mathrm{GeV*}$, the production electron and positron bunches are split opart in the Bean Switchyard by a bending magnet and folkw the curved, terrain-following ares around to the IP, where they collide. Each outgoing beam is then steered onto a benm dump, and the cycle begins agnin. The repetition rate is $120 \mathrm{~Hz}$. Sore description of the accelerator and the accelerator-detector interface and interaction will be provided in Appendix C.

\subsubsection{Spin Transport}

To preserve the longitudinal polarization of the electron beam, a series of spin rotation solenoids were installed. The cote immediately upstrean of the North damping ritug is crucinl, as it rotates the efectron spirs into the vertical plane so that they will not precess during their time in tlie ring. Any precesion of this type would result in a large decrease in the average polarization of tbe beam, since particles witb different energics bave different spin-preccstion rates. Since the time spent in the dimping ring is celatively long, any non-zero precession rate would have a long time to depolerize the bearn.

The other two spin-rotator walenoidy were used in 10y2 to provide an arbitrary orientation of the glectron spin at the IP. However, in 1993 the operational mode changed to so-called "flat benms", whose verticnl size is much amaller than the borizontal[209]. Since the spin rotator solenoide downst ream of the damping ring would mix the borizontal and verical beam motions, potentially destroying the small vertical spot, they were turned ofI. Instead, the fact that the SLC aros have a betatron oscillation frepurncy very close to a spin-precession resonance frequency is urod to manipulate the

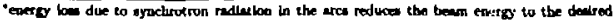
vilue of $\mathrm{H}_{z} / 2$.
} 


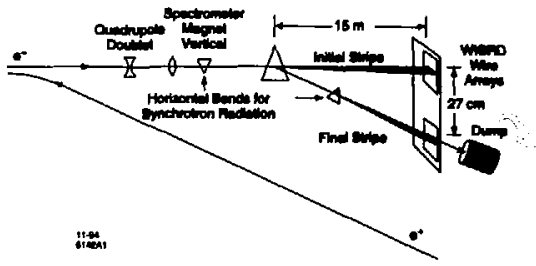

Figure 4.3: A diagram of the WiSRD chergy spectronjeter, showing the beam components and the WiSRD cletertor.

spin orientation[15j]. This has been exi remely sucicisin].

\subsubsection{Beam Energy Measurement}

The bearn edergy is measured on every pulse by two spectrourctera that are pleced jux prior to the outgoing beam dumps where the spent beams arrive after the collidion. The axtual energy measurement is performed by deflecting ench beal horizockelly, then vertically by a preciscly-ealibrated bend magnet, then boxinomally agin. The horizontal bende produce two kynchrotron radiation swaths whoee positions are measurct by the Wire Imaging Syndirotron Radiation Detector (WISRD)[154]. The vertical distance between the two stripes is inversely proportional to the beem energy, which can be extracted given the integrated field of the precision bend and the distence to the detector. A schematic view of the WISRD spectrometer is shown in Figure 4.3. The center of mases energy for the 1993 run was $91.26 \pm 0.02 \mathrm{GeV}[47]$ ].

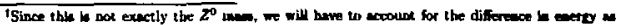

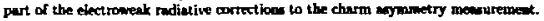




\subsubsection{Beam Polarization Measurement}

All of the electroweak esymnetries measured at the SLC dejend op a precise knowledge of the longitudinal polurization of the electron bean. Thu polarization is anessured immediatcly downstrum of the SLC IP by a Compton Polarimeter, which uses the difference in t]e Compton scattering cross sections[155] for the $J_{2}=\frac{3}{2}$ aud $J_{z}=\frac{1}{2}$ combinatious of tircularly polarized light and the longtudinally polarizexi electron beam to extract tbe electron beam polarization. The ineasurement is baned on the dependence of the scattering crows bection $\left(\sigma_{C}\right)$ on belicity and on the electronphotw collision angle in the center-of-mass frame ${ }^{t}$ When the electron-photon collision is boosted back into the laboratory frame, the differences in scuttering probability as n function of scattering angle become olwervable as an asymunetry in the scattered clectron energy spectrum. The shape of the usymmetry function does not depend on tle polarizations of the electron or photon beams, as it is only $n$ function of the cnergy in the center-of-auss system. The unknown electron bean polarization $P_{\mathrm{e}}$ can be extracted from

$$
A_{\text {treas }}=\frac{\sigma_{C}\left(J_{z}=\frac{3}{2}\right)-\sigma_{C}\left(J_{z}=\frac{1}{2}\right)}{\sigma_{C}\left(J_{z}=\frac{3}{2}\right)+\sigma_{C}\left(J_{z}=\frac{1}{2}\right)}=a_{d} P_{z} P_{7} A_{C}(E)
$$

where $P_{7}$ is the (measured) laser polarization, $E$ is the (mensured) scattered electron cuergy, $A_{C}$ is the (caleulated) Compton scattering asymuetry, $A_{\text {mes }}$ is the observed Bsy rnsets; and $a_{d}$ is the (culculated) anabyzing power of the detect of which actually measures the scattered electron energy and deternincs the nymmetry. The errors on the bean polarization measurement come primarily from uncertainties in the nct circular polarization of the laser and the analyzing power of the detector.

The polarimeter bystem[156] is shown schematically in Figure 4.4. The photon beam is provided ty a frequency-doubled YAG laser which produces $2.33 \mathrm{eV}$ photons which are transportal down into the SLC to collide with the $45.6 \mathrm{GeV}$ electron beam at the Compton IP, which is 33 meters downstream from the SLC IP. The ejectron energy is measurta $b y$ a spectrometer consisting of an analyzing bend magnet and a multi-chamel Cerenkov detector. A tremendous amount of effort has been

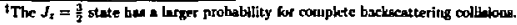




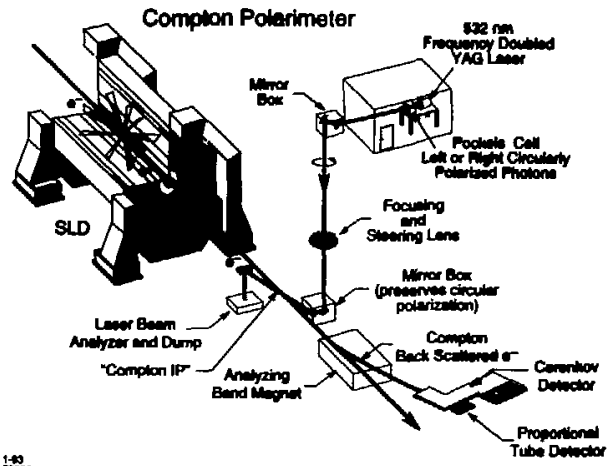

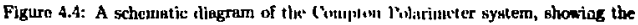
positions of the components relative wo the' $\$ 1.11$. Whiveter.

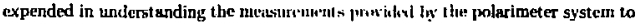
a precision of better than $1 \%$. The susills of this witk ure strown in a somewhat

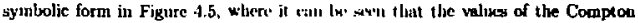

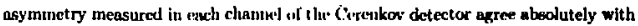
the theoretical calculation and a Monte Curlu simulation of the detector respones to within $0.5 \%$, channel-by-chaunel.

The measured polarization for the 1993 run is $63.0 \pm 1.1 \%$. 


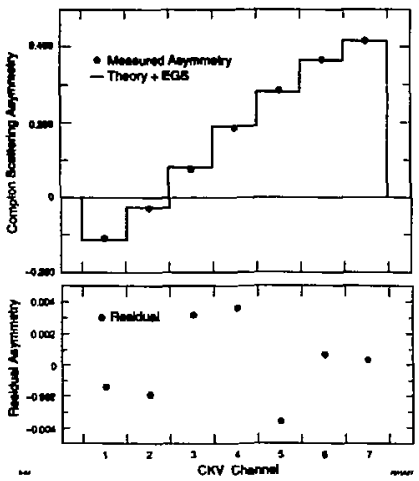

Figure 4.5: The measured Compton asyminatry, commared wth the theoretical calcuLetion as modified by the EGS[213] simulntion of tilu Cerenkov chetector response. The lower plot shows the ubsolute channel-by-chunicl risidus! between the measurements and the calculated values.

\subsubsection{SLC Performance History}

Construction of the SLC began in 1083 and concluded in 1987. After a sorrewhat difficult commissioning period, the lirst $Z^{0}$ loton ever produced in $e^{+} e^{-}$collisions was observed by the Mark II detector in April of 1989. Over the course of two runs spanning 1989 and 1990 the Mark Il recorded $826 Z^{0}$ decays, with peak luminosities of the SLC rusching $4 Z \mathrm{~s} / \mathrm{hr}^{5}$. The Mark II era ended on Nonerober 21, 1990.

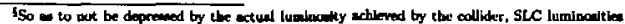

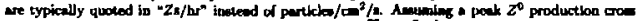

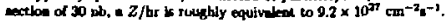




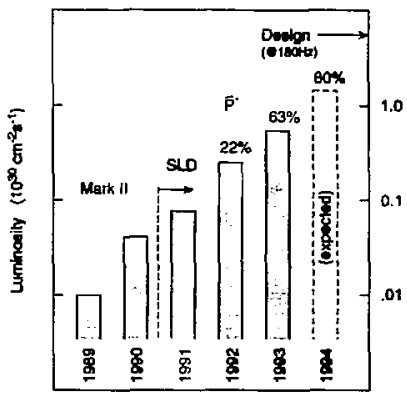

Figure 46: The history of the SLC Lutinoxity performance, along with the achieved polarizations to date. The urrow labeled "Design" is the propowed SLC luminowity, with the accelerutor running at $180 \mathrm{~Hz}$ colliding bunches of $7 \times 10^{10}$ particles.

The SLC Large Detcetor (SLD) progratu began with an cagineering rm in 1991, during wbich luminosities of $6 \mathrm{Zs} / \mathrm{hr}$ were achieved during $60 \mathrm{liz}$ operation. The improwemeut in luminosity is mostly due in this instance to the increased magnetic field providerl by the new: superconducting final qundrupole triplets which perform the funel focussing of the beans just prior to the IP. In 1992, SLD had its first physics running, recording approximately $1000 Z^{0}$ decays with unjolarized beams, and $10,000 Z^{0}$ events with an average polarization of $22 \%$. Peak luminosities werc around $30 \mathrm{Zs} / \mathrm{hr}$. During a machine physics program at the conclusion of the 1992 run which was ained at studying final focus systems for the next generation of binew colliders[158], it was realized that the linac was capable of transporting the nuturelly fat' beam from the damping rings without much growth in size due to sdded racolom

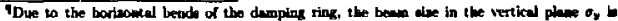
typleally much inaller than $\sigma_{x}$ unkar the $x$ ad $y$ motion ure alined.
} 


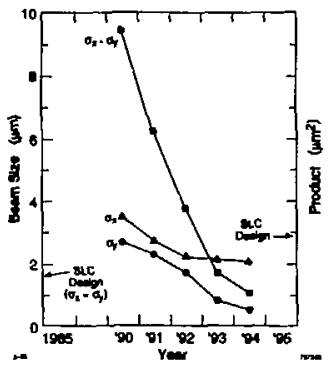

Figure 4.7: The listory of the SLC beain size/area, slowing the horizontal and vertical sizes of the berns, and the combined bean aren. The design area is also shown.

beam uotion. This reduced the aren of the leam erose section by approxiunately a factor of two with essentially no changes to the accelerator, and was choeen as the default operating mode starting with the $1993 \mathrm{~min}$. The SLC produced approximately $50,000 Z^{0}$ bosons that were recorded by the SLD during the 1993 rum, and peak luminosities approeched $60 \mathrm{Z} / \mathrm{hr}$. A history of the luminotity provided by the SLC is shown in Figure 4.6. The cxponential growil of the luniursity continues, as, at the time of this writing, peak luninosities near $90 \mathrm{ZB} / \mathrm{hr}$ have been seen". This next jump has been made possible by the installation of new final focus elements that canet some of the clurinnatic aberrations that limited the vertical sper size. The historical progression of spot sizes in the horizontal and vertical plaues is shown in Figure 4.7. The small, st uhle luminous region of the SLC will play a large purt in the analysis of this thesis. The history of the integrated luminosity for the 1991 through

\footnotetext{
It is lxartening to thut the SLC in now capable of producing in a day awore $Z^{0}$ events thas the Alark 11 tocorded in ice zrueling two geang of rumbing.
} 


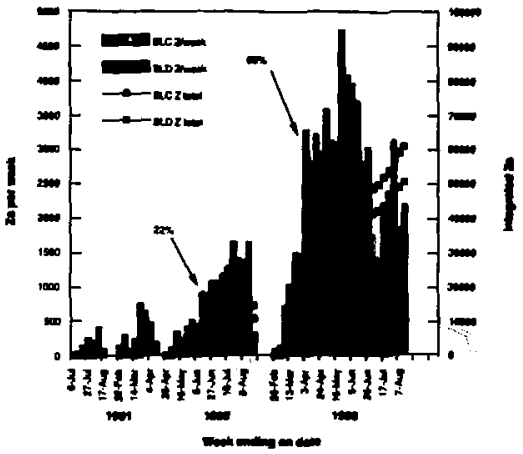

Figure 4.8: The Integrated Luminusity for SLD nuning from 1991 to 1993, dieplayed as luminosity per week (left axis) and total luminosity (right axis) as delivered by SLC and recorded by SLD.

1993 runs of the SLD experimeut is shomn in Figure 4.8.

\subsection{The SLC Large Detector}

The SL.C Large Detector (SLD) was fint proposed[150] in 1984 and was compteted in 1990. It was roled onto the SLC bcanline in time for the sutnmer 1991 engineerine run, and has been recoxding data ever since. The SLD poweses the cylinder-endcap geometry typical of most collider detectors. An isonetric view of the SLD in abown in Figure 4.9, where the reparate elements which make up the detector 0 \& whole are labcled. The individual detector elements, beginning at the center and working 


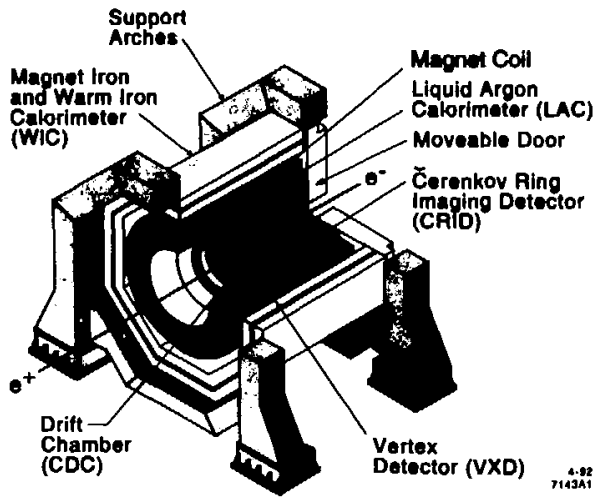

Figure 4.9: An isowetric view of the SLD detector, showing the layout of the detector componemLB.

outwards, are:

- The Vertex Betector (VXD)

- The Lunithority Monitors (LUM)

- The Central Drift Chamber (CDC)

- The Cerenkov Ring Imaging Detector (CFJD)

- The Liquld Argou Calorimeter (LAC)

- The Solenoidal Mecuet. 


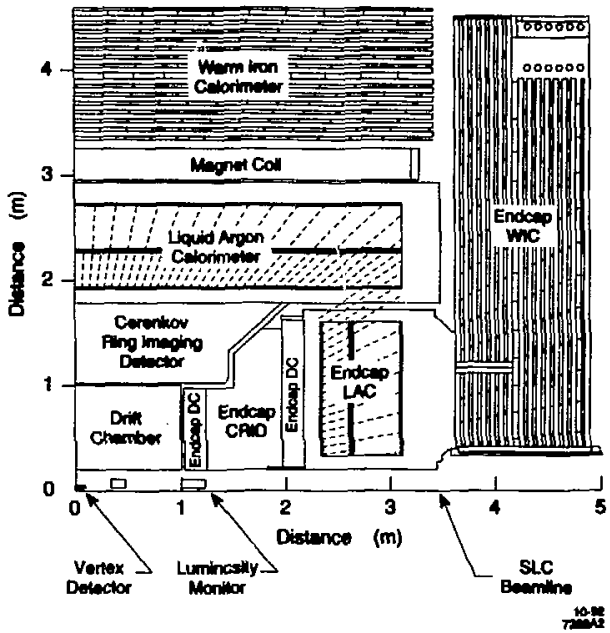

Figure 4.10: A quadrant view of the SLD detector, giving the overall dimensions of the delector components.

- The Warn Iron Caborimeter (WIC)

The endcaps which complete the bermetic conerage of the detector are organized in a similer fashion, except that the VXD (and the solenoid) ha no endcap composents. Euch of thene detector elements will be described briefly in the following sections with 
cmphesis on the barrel systems, as the endcaps (excepting the LAC) were not urod in these andyes. Special attention will be focused on the tracking aystems an their performance lies at the heart of the analyes prosented in this thexis. A quadrant view of the detector including the physical dimensione in showin in Figure 4.10.

The SLD coordinate system taked $z$ to 1 a along the beam axis, with $z=0$ defined $\omega$ the center of the CDC. The ry plane to perpendjcular to the beam axis, with the $x$ axis parallel to the ground. The anghe $\theta$ will be uod often in this thesin, and in defined of the angle bet ween a vector or line druwu from the IP to the object in question and the bean axis. We will define positive $\cos \theta$ to mean that the angle in quextion is arraller than $90^{\circ}$ when measured with respect to the outgoing electron beam, alt bough the official coordinate system of SLD signs this in the opposite manner.

\subsubsection{The VXD and Beamplpe}

The beampipe section ahich lies at the very center of the SLD is Iasbioned from a thin beryllium cybider. This section of pipe was made $25 \mathrm{~cm}$ long so that the full tracking volume of itu SID would be preceded by a mininum of waterial. The beampipe's outer diameter is $25 \mathrm{~mm}$. Including the VXD cooling jacket, the total thickness is radiation leugths hefore the first layer of the VXD is $0.71 \%$, which is amaller than the thickness of cach VXD layer. This material is kept as small an possible to minimize the amount of scuttering earh track undergoes belore the first position measurement can be performed.

The VXD uses Chrrge-Coupled Devices (CCDs) as the modiun for detecting the Leposition of ionizntion from through-going charged particles. Since a single CCD is conposed of a large uumber of tiny jixels, a detector based on this technology is a source of three dimeusional spare juints along the track trajectory.

The VXD is constructed[160] from sixty $9.2 \mathrm{~cm}$-long ladders arranged in four concentric cylinders which are beld in plsce by a beryllium shell. An end-on view of the detector is uhown in Figure 4.11. Eiglit CCDs are mounted on each ladder, with fow on each wide to maintain full coverage in $\cos \theta$. Oue can from Figure 4.11 that most of the tracks passing through the VXD will uiquire two VXD bisa. Some 


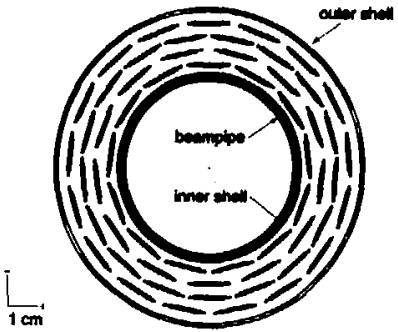

Figure 4.11: An end view of the VXD, showing the four layers of CCD-earryins ledders. The gaps between ladders result in what is effectively a two-leyer trecking device. The innermont layer is $28.5 \mathrm{~mm}$ from the center of the detector.

tracks can hit noxe than two CCDs, und the average number of VXD hite per track is 2.3. Two hits are pusible on any trock for $|\cos \theta|<0.74$. The inner layer of CCD. is $29.5 \mathrm{~mm}$ from the IP, and the outer layer is $41.5 \mathrm{~mm}$ axray. Each layer comprien. $1.1 \%$ of a radiation lesigth $\left(X_{0}\right)$ in material.

Each CCD is appruximately $1 \mathrm{~cm}$ square, and contains $375 \times 578$ pixels, each 22 hm-square. Each pixel has a depletion depth of $20 \mu \mathrm{m}$, which allowis excellent position revolution even for traves passing through the detector at lurge dip angles. This fine granularity provides robustness against tracking inefficiency duc to large backgrounds in tire detector. This is 2 ureful feature in the SLC environpent, slnce the skow romdout apeed of the CCDs necessitates a readout time of approximately $160 \mathrm{~ms}$ (19 beam crowings), during which time any charge deposition in the detector is resorded. Even in this high-noise environnent, occupancies above $0.1 \%$ are extremely rare. Upon installation, it was discovered that two of the ledders and an anorted CCD or two were completely inoperative due to inaccenible bad connections. This resulted in 
approximately $4 \%$ of chanpels remaining inoperative for the lifetjme of the detector.

An internal alignment of the poxitions of all of the CCDy rulative to their lublers and the relative pasitions of the ledders theunselves was pesformed uajug changed tracks from $Z^{0}$ hadronic decays. To check this procedure, tracks with three VXD hits were used to obtein the intrinsic position resolution (ircluding alignoment errors) of exch hit. The messured singlo-lit resolutions are $5.5 \mu \mathrm{m}$ in the $x y$ plane, and 5.5 $\mu \mathrm{m}$ up to $9 \mu \mathrm{tn}$ for centrol tracks and those with $|\cos \theta|>0.55$, respectively[160]. These values are consistent with those lound by using the two-track miss distance in $Z^{0} \rightarrow \mu^{+} \mu^{-}$and $Z^{0} \rightarrow e^{+} e^{-}$events fitted with the VXD hits only. The slight degradation in the resolution at larger $\cos \theta$ is most likcly due to radial aligntment errors and homing of the CCDs.

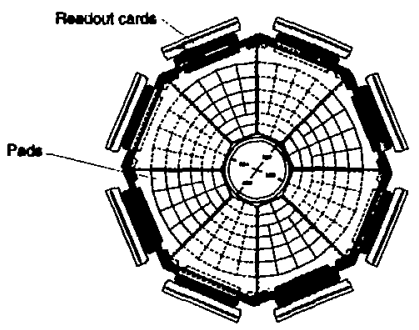

Figure 4.12: $A$ beam's eye view of the Luminosity Monitor (LUM), sbowing the pad structure and the readunt cards. The inner diameter is indicated in the center. 


\subsubsection{The LUM}

The luminosity monitors for the SLD are silicon-tumgren calorimetery arranged in a pad configuration surrounding the beampipe[1G1]. Their primary purpan se to meesure the abeolute integrated luminosity by recording all nonell angle Bbubha scattering crents. The inner edge of the fiducial acceptunce is defined by a tungeten naak that covers the regiou of $|\cos \theta|<25 \mathrm{mr}$. The peds ure urrenged into $\mathrm{n}$ projective tower geometry. A beam's eye view of the LUM is shown in Figure 4.12, where tbe tower segmentation cuu be meen. Each tower is divided longitudinaliy into an EM1 section of 5.5 radiation lengths in thickness and an EN2 section of 15.6 radiation length The energy resolution has been measured[162] to esscutially agrce with the design of $3 \%$ at $50 \mathrm{GeV}$.

\subsubsection{The CDC}

The CDC is the primary tracking detector for : ise SLD[163]. It is constructer in the form of a cylindrical annulus of inscr radius $20 \mathrm{cru}$, puter radius $1 \mathrm{~m}$, and length 2 m. The actiwe clements are $\mathbf{5 1 2 0}$ serise wires interspersed throughiout the chamber volume. Tile ahell structure which bears the tension of the wires wus designed to be as thin as poesilile. It consists of "disbed" 5 min-thick siuminum endplutes and inter and outer cylinders macle from an aluminum sbect-Hexcel fiberbaard laminate. The inner cylinder las $1.8 \%$ of a radiation length of material.

The sense wircs are arranged in 80 lasers wich are organized tuto 10 superlayers of 8 wires each. Six of the stiperlayers have a 40 mrad stereo angle with respect to the beam axis to allow a messurement of the z position of the track bits. A subection of the CDC endplatc slowing the layer geonetry is shown in Figure 4.13. The superlayers are broken into jet cells, where each of sense wires is radially oriented". The wire layout of a single cell is shown in Figure 4.14. The field-ahaping and guard wires consist of $150 \mathrm{~J}$ m diameter gold-coat ed aluminum wires, and the sense wires are $25 \mu \mathrm{m}$ dinmeter gold-conated tungten. Each set of sense aud guard

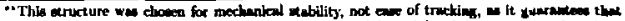

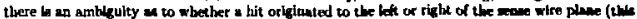

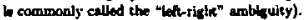




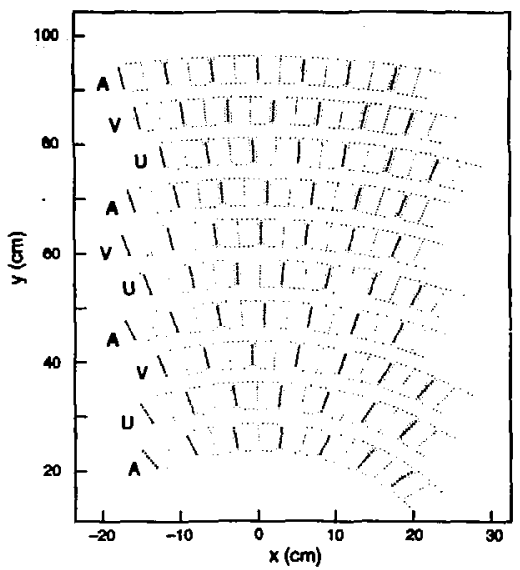

Figure 4.13: A portion of the CDC endplate, showing the layout of axial (A) and sterco $(U, V)$ layers.

wres is unemb itd into individunl Lexan blocks which can be precisely preitioned and tensioused in situ in stots in the ulumium endplate.

To read out the signals fron the chasiver[164], the charge from each sense wire is clocked on each bean crowing into a switched caperitor array, which functions as an analos uremory unit (HAMU). If the chamber is to be read out, the charge is digitizod and aent up to a FASTBUS waveform wmpling module (WSM), which performs zero suppression, corrects esch HAMU chanpel for lintarity, and subjects 


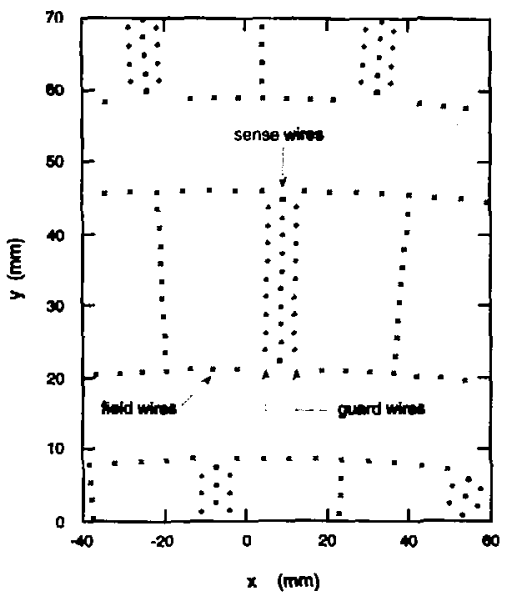

Figure 4.14: A dikstam of an individual CDC cell, showing the sense, guard and fied wire layout. The two sense wires th the rind of each rou are dummy wires and are not read out.

the date to waveform- finding algorithrn which extracts, for each wire end, the time, chargo, leight, and width of each pulse found in the data. Both ende of the chamber are instrurpented, so that the $\mathrm{x}$ coordinate of ach hit can be moarured to approximately $2 \%$ of the wire length using tbe charge division of the pulne.

The gas mixture wes choeen to provide maximum preclaion on the drift ditance 
mesurement. To thin end, $\mathrm{CO}_{2}$ was chosen at the primary ges in the mixture, os in has a bow drift velocity and a kow diffusion constant. The kow drift velocity aljows higher remitution on the shape of the arriving pulse for a fixed anpling time, and the bow difusion limits the pmeariog of the pule over long dintances. The other components of the ges are: bobutanc, added es i quencher; argon, added to iucrense avanche gain; and trace amounts of water to supprese the efrects of wire sging[165]. The composition in percent is $\mathrm{CO}_{2}: A r i s o b u t a n e: \mathrm{H}_{2} \mathrm{O}: 155: 21: 4: 0.2$. The drift vekxity of the final gas mixture is $7.9 \mu \mathrm{m} / \mathrm{as}$ at the mean drift 6 eld of $0.9 \mathrm{kV} / \mathrm{cm}$. It is worth noting that the drilt velocity of the ges depends strongly on gas density, gas compaition, and the electric field in the drilh region. All of these must be monitored in order to guarantee stability and precision of the drift distapce measurement. The bocal resolution. which is celculaled by the difference in residuals of adjacent bits on a track within individual drift cells, awd the globel resolution, which is given by the residunis of all of the hits on a track, are sbown in $\mathrm{Fi}_{\mathrm{h}} \mathrm{r}$-ut $4.15 \mathrm{as}$ a function of drift diotance from the wire plane. The difference is locel and globel resolutions can be attributed to small inter-cell alignment errorst". Also plotted is the resolution curve one would expect for diffusion, given a rosolution of 68 $\mu \mathrm{m}$ at $1 \mathrm{~cm}$. The moan resolution of the hits between 0.5 and $2.5 \mathrm{~cm}$ is $82 \mu \mathrm{m}$, making this the most precise lerge drift chanber eser constructed.

Two other mesurres of the chamber performance on the local level are the hitfinding eficiency and the twa-hit resolution. The hit-finding efficiency is defined as the fraction of time a hit in one of the $\mathbf{8 0}$ sense wire layens is found on thectu which pased through that leyer. This is slrown in Figure 4.16, along with the MC expectation for the same quantity. Iligh bit-finding efficiency and good acreenent with the MC have been achieved. The two-hit reolution is $j ;:-i b$; the minimum resolvable separation between bits on adjacent tracks. This is shown in Figure 4.17, where an efficiency of $50 \%$ is obtained at a hit separation oi $1 \mathrm{~mm}$. This resolution is determined by the length of eacti pube after diffunion, elc, and the opecific portion

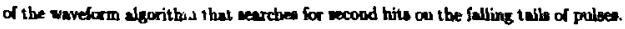

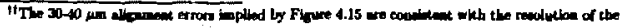




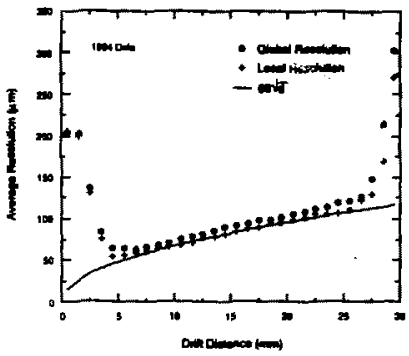

Figure 4.1 is The bocal and global GDC drift distance readution mesenred with trwde in hadronic events from the 1994 date sample. Aloo shown is the renolution curve expected due to diffusion effects for the specific $\mathrm{CDC}$ ges composition. The difiuion turve has been normalized to gite the correct minimum resolution.

\subsubsection{The CRID}

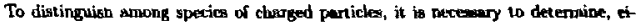
ther directly or indirectly, the masey of each particle under scrutisy. With particies moving at relativistic speeds, it becouns a much simpler matter to meanure the momentum of a particle and its velocity, thereby deternining the mass indirectly*. Thle has been accomplished in pust collider experiments by prociec menurements of the travel times of particles between two fixed points ("time-of-fight"), and measirements of the bonization per unit puth length through a gus ( $\left.d E / d x^{n}\right)$, which in related to the Lorentz $\beta \gamma$ of the particle). Cerenkov detectors, which mesurn the Loremz $\beta$ of

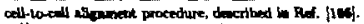

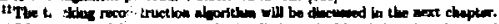

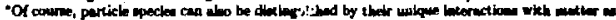

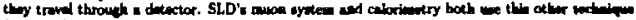

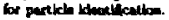




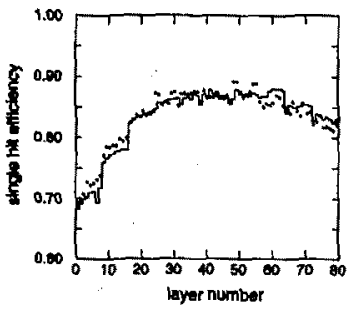

Figure 4. I6: The efficiency for finding a single hit on a track which puses through a given layer in the CDC. Shown are the efficiencies in the data (points) and the MC sinulation (solid histogran). The detailed geometry of the cell louktiont determines the layer-to-layer snriations.

a particle, are heavily hsel in fixet-target experiments for farticle identification but hove only recently been adented for the cylindrical geonetry of a collider detector. The SLD CRID is one of anly two such detectors crer constructed These devices allow measurements of the Cerenkov angle for all perticles in an event whose velocity is targe enough to allow then to cmit Cerenkov rediation. The setive elements of the CRID detector rTe long time projertion clambers (TPCs) which drift and captwe the photoelectrosu that have been liberated from the TPC gis volume by the Cerenkov photons. Tlic CRID has two radiatory to generatc Cercnkov photons, a liquid $\left(\mathrm{C}_{6} \mathrm{~F}_{1} 4\right)$ and a gas $\left(\mathrm{C}_{5} \mathrm{~F}_{1} 2\right)$. Photons from the liquid radiator impinge directly on the drift boses, while a system of 400 mirrors locuses the photons from the gas radiator bark onto the photo-sensitive medjum. Given the direction and momentum of a particle as determined by the CDC, the radius of the "ring" of photoelectrons cresled by the cone of Cerenkov photons determines the Cenenkov angle and bence

\footnotetext{
The other a the DELPHI Rthy Imanging Cerenten (RICH) detector[167].

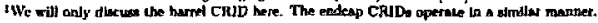




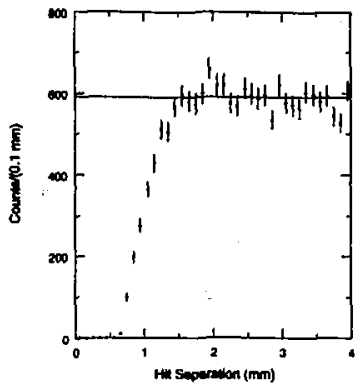

Figure 4.17: The number of bits found in a single CDC layer as a function of the distance ofter the first hit. The horizontal liue is drawn at full hit-finding efficioncy. The $50 \%$ efficiency point correspouds to a hit separation of $1 \mathrm{~mm}$.

the particle velocity. A schernatic view of the CRID detector is shown in Figure 4.18. At this time, the GRID is beconing well-enorgh understood to be useful in physics analy:ces. The observed number of photorlectrons and the loral resolutions on the unessured Cerenkov angies have been meusurad to be close to the derign values.

\subsubsection{The LAC}

Calorimetric energy measurements are provided by the LAG, a sampling calorineter whose besic module consists of lead plates innersed in liquid argon[169]. The argon is the active medium, as it is ionized by chargod particles pazsin' through the calorimeter. The lead serves to induce particle showers and as the coll stion medium for the charge liberated in iotizing the argon. The basic structure of the LAC is shown in Figure 4.19. The layers of lead are broken up into alternating grounded 
phates and tiles held at high voltage; it is here where the churge collection cecurg. As the lead is stacked, the tiles are arranged intu projective towers whose longitudinal depth depeads on how many layers have beven ganged together for readout.

The LAC is segunented into two layers, the electromagnetic (EM) and balronic (HAD) sectious. The Eil section is formed of $2 \mathrm{~mm}$ thick iesd plates sepurated by $2.75 \mathrm{~mm}$ of liquid argon, and is subdivided into two layers, EMI (6 $X_{0}, 0.24$ interaction lengtha $\left.\left(\lambda_{0}\right)\right)$ and EM2 (15 $\left.\lambda_{0}, 0.60 \lambda_{0}\right)$. The HAD section is formed of $6 \mathrm{~mm}$ thick lead plates, but the $2.75 \mathrm{~mm}$ argon gap is muintained. The HAD wection is also subdivided into HADI $\left(13.9 X_{0}, 1 \lambda_{0}\right)$ and HAD2 $\left(13.9 X_{0}, 1 \lambda_{0}\right)$. The EM towers subiend one quarter of the solid nngle of the HAD toners, so that each set of four EN towers is backed by a single HAD tower. The EM sections contain approximately $99 \%$ of the energy from a $45 \mathrm{GeV}$ electron, while the LAC as a whole contsins $85-90 \%$ of the total energy in a hadronic $Z^{\circ}$ decay. The emet By resolutions for the LAC have been measured[170] using $\mathrm{e}^{+} e^{-} \rightarrow e^{+} e^{-}$and $Z^{0} \rightarrow$ gi events to be $12 \% / \sqrt{E}$ and $65 \% / \sqrt{E}$ for the EM and HAD sections, respectively.

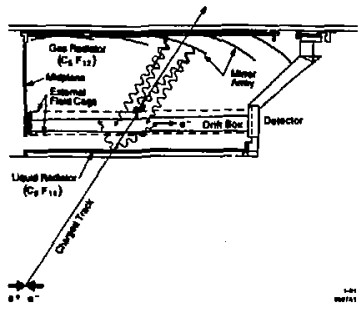

Figure 4.18: A schematic diagram of the principle of CRID operation. A charged perticle entering the likquid radiator emits Curenkov photons which impinge on the dift boxes containis a ploto-sensitive medium. Cerenkow phouns from the ges radiator are focused back onto the photo-sensitive medium for detectlon. 


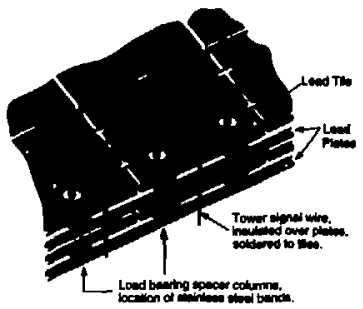

Figure 4.19: The internal structure of the LAC, showing the alternate layers of plates and tiles which form the readout towers.

\subsubsection{The Solenoidal Coil}

Surrounding the LAC is the SLD solenold, an aluminum core magnet. It prowides a field of 0.6 Tesle along the beam axds. The field has becn rapped[171] to be uniform to $3 \%$ over the tracking volume of the CDC; the lawest order polynomial expansion to the non-axial field in a finite solenold is used during track reconstruction to paiametrise the field aun-unlformity. The polynomial agrces with the the measured field to better than $0.05 \%$ over the CDC volume.

\subsubsection{The WIC}

The WIC serves four functious within the SCD: Hux return for the solenoid, a backing calocingter to neasure the residua badronic energs which lias leaked out of the LAC, a moon-identification system, and the structural support for the rest of the detector components. The WIC is made of 18 lavers of lartoci (limited-streamer) tubes sandwiched between $5 \mathrm{~mm}$ thick steel plates[172]. The tubes are instrumented with square pad readout for caloximetric purpoes and long strips for reading out the individual tubes in arder to use the WIC as a muou tracker. The WIC geondetry is 


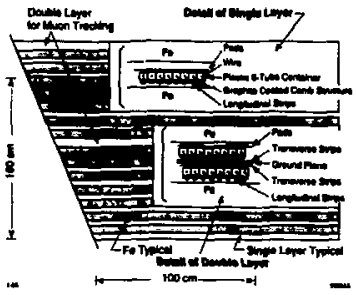

Figure 4.20: The WIC structure, slowing the single layers containing longitudinal strips for muon tracking as well as pad tower resdout. Also shown are double layers with crossed strips for tracking in the other planc.

shown in Figure 4.20. The WIC stripw which provide the muva-tracking informatton are arranged in tho separate arrays $90^{\circ}$ from each otber to chable the trajectory of a muon in thro dimensions. The cndcap chambens in particular have half of the strips oriented vertically and half horizontally. This information will be useful in the context of the bxckgrounds discussion in Appendix C. The barrel W7C has proved to function as intended and has been used to identify muons from beavy quark semlleptonic decays[7; 3$]$

\section{- 4.2.8 The SI.D Detector Simulation}

The interactions of all purticles with the materinls of the SLD are simulated by the GEANT 3.15[173] package of routides. The compouition of each of the detector volumes is specified, and the simulation deposits the appropriate amount of enorgy in each volume, ecatters through-going particles by angles consistent with the materinl thicknes, and allows electromaznetic and hadronic showers to occur. Hadronic interactions are simulated by the GHEISHA package; electromagnetic showera are deposited in the detector using a parametrized shower shape rather than running a 
detailed stmulation.

To simulate the occelerator-induced beckgtounds that arise in the varions detector elements, all of the signalu trom random-trigger events clowe in time to the $Z^{0}$ decers are overlayed on top of the geperated Monte Carbo events. This is an attempt to insure that the proper luminosity-meighted background levelo are properly simuleed.

In addition, the obererved complement of dead chandeln is reproduced in the winulated detector response, so that the time-dependene configuration of the simalased detector matches that of the real SLD over the course of the run.

A detailed description of the SLD modifications to the heavy flavor decay packere in JEISET 6.3 can be found in Ref. [166]. 


\section{Chapter 5}

\section{Tracking System Performance}

As will be seen in the next chapters, a thorough underatanding of the minute details of the tracking syatem is crucial to the andyoes presentrit here. This chapter gives an overvipw of the excellent performance of the SLD tracking system and our understanding of lts workings, which is evidenced by measurements of data events as well as our ability to obtain agnement between data and the Monte Carbo simulation. In the previaus chapter describing the detector, various details of our underatandiug of the intrinsic local resolutions of the CDC and VXD were given. Below, will discuss the global penformance of these detectors. As charged tracks are reconstructed using both the VXD and CDC, thls global perfornuance directly impacts the physics analyses that rely on the precision of the tracking system.

\subsection{Track Reconstruction}

Charged track reconstruction begins in the individual drift cells of the CDC*. A "Vectored-Hit Finder" searches for straight or slightly-curved strings of hits on the eight sense wires in esch cell. Shorter combinations not contained within longer ones are also sought. Fach of these mininture track segments is neduced to a vector giving its direction and position. These serve as the input to the patter recognition software,

\footnotetext{
- As the VXD ooly provides two hita per track, it le not powille to do axand-alone uncling within

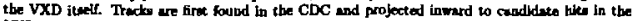
VXD.
} 
which atternjts to find actual tracks in tbe CDC. This stage begins by boikg fint at patterns of hits which form circles in the fotr axjal leyers, then proceede by addine hits from the stereo layers to form long tracks, Sborter track not conkeined within longer ones are slso foudd(174), down to tracks spenning 4 CDC superlayers. Ather the tracks are found, the hitn which they contein are recorded, and a preliminary track fit is pefformed. This fit pooduces initial track parameters which can be input into the actual iterative track fitting algorithm. The output of the track fitter in a track defined by two sets of track parameters, ode set evaluated at its innermoct radius, one at its outermost. Extrapolation inward to the VXD or outward to the CRID, LAC, or WIC begins with these sets of track parameters.

The CDC performance can tre studied by wing the paira of tracks frotn $Z^{0} \rightarrow \mu^{+} \mu^{-}$ and $Z^{0} \rightarrow e^{+} e^{-}$events, as well ws those from cosmic rayst. The neesured resolution for tracks found in the CDC only for the quadities relevent to physios analyoes are shown in Toble 5.1. For tracks to be used in andyses requiring precise knowledse of the track trajectory pear the IP, the resolutions of doca ${ }_{\tau y}^{\prime P}$, the dirtance of closeat approach to the IP in the plane transwerve to the beam axis, docal? , the dist ence of closest appronch to the IP along the bean axis, and $\lambda$, the polar angle of the track with respect to the vortical, are critical determinants of how well tracks can be extrapolated to the VXD in an attempt to find nsociated hits. The other parameters shown in the table are the resolution on tbe track direction $\phi$ in the $x-y$ plene, and the infinite monentum (a) and momentum-dependent $(b)$ terms in the momentum Iesolution, where $\sigma\left(p_{1}\right) / p_{t}^{2}=\sqrt{a^{2}+\left(b / p_{1}\right)^{2}}$. Plots of the $z-y$ and $r-z$ two track mixe distaners are shown in Figure 5.2. Except for the momentum, the resolutions for a single tack are deconvolved from the distributions of the differences between the two tracks for each of the messured quantities. The larger resolutions for those quantitiea measured in the $r-z$ plane are typical of chambers that use stereo wire hyers to measure the track positions along the cylindrical axis of the detector. As the VXD does not have a large enough kever arm to mesuure track momentum itgelf, it mus rely on the CDC measurement as a starting point. A plot of $q / p$ for $Z^{0} \rightarrow \mu^{+} \mu^{-}$

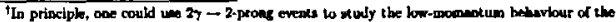

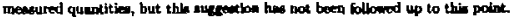




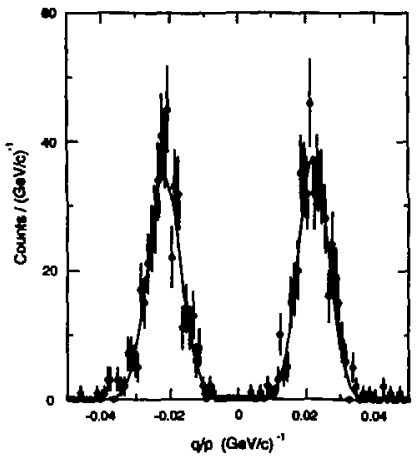

Figure 5.1: The signed inverse momentum of muons in $Z^{0}-\mu^{+} \mu^{-}$everts as determined by the CDC alone. The golid curves are gnusian fits to this distribution. The muons uged for this meesurement have sufficieatly small polar angles that the distribution is sell-spproximated by a gausian.

and $Z^{0} \rightarrow \mathrm{e}^{+} \mathrm{c}^{-}$esents is given in Fig 5.1 shows the expected gaussian shape. The momentum resolution of the $\mathrm{CDC}$ is $\sigma\left(p_{\perp}\right) / p_{t}^{2}=\sqrt{0.0050^{2}+\left(0.010 / p_{1}\right)^{2}}$.

To match the tracks reconstructed in the CDC to lits they possibly left behind in the VXD, the tracks are extrapolated inward to the outermost layer of the VXD. The extrapolated track exrors give an indication of an appropriate search region in which to look for VXD bits that could have belonged wo that track. A Billoir fit[175] is performed for each poesible combinations to select those VXD hitg (if any) which

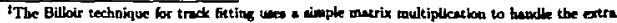

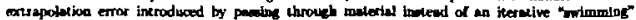
method, which seps the tracks along their belicul peth and coneiders makerial on a much more beal 


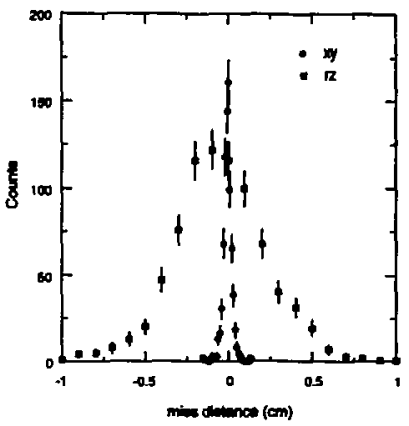

Figure 5.2: The two-track miso distances of muons in $Z^{0} \rightarrow \mu^{+} \mu^{-}$events as determithed by the CDC abone. The solid points represent the mesoured diatribution in the $z-y$ plase, the open squares represent that in the r-z plane.

provide the best match to the track. Hits are not allowed to be ahared by tracks. A find pars is made using a wenk vertex constraint to attenpt to link tracks to thowe regions of the VXD where ouly one hit is avilable due to the dead CCD.

The efficiency for linking "quality" CDC trocks' to hits in the VXD is shown in Figures 5.3 and 5.4a-b plotted ageinst track momentum, track polar angle $\theta$, and track azimuthal angle $\phi$. Alos strown in these plots are the same quancitiex and the fraction of the links that are incorrect as predicted by the MC. One can see from these plots the quality of the links, as well as the quality of tbe MC simulation, which ayrees with the data to better than $1 \%$ for quality trecks. Figure 5.5 shows the fit quality $\sqrt{2 X^{2}}-\sqrt{2 n_{1-a !}-I}$ of the combined CDC+VXD Billoir fit for data and MC. The bacle.

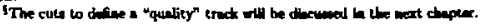


Thble 5.1: The resolutions messured for various track parameters with the CDC and CDC+VXD tracking systems. The trisibles are defined in the text. Resolutions quoted are those from single tracks, on deconvolved from the two-track difference dintributionn.

\begin{tabular}{|c|c|c|}
\hline Paraneter & CDC Only Resolution & CDC+VXD Resolution \\
\hline doca $_{s y}$ & $150 \mu \mathrm{mi}$ & $11 \mu \mathrm{m}$ \\
docars $_{r z}$ & $1000 \mu \mathrm{m}$ & $38 \mu \mathrm{m}$ \\
$\phi$ & $0.45 \mathrm{mrod}$ & $0.32 \mathrm{mrad}$ \\
$\lambda$ & $3.7 \mathrm{mrad}$ & $2.2 \mathrm{mrad}$ \\
$a$ & 0.005 & .0026 \\
$b$ & 0.01 & 0.0095 \\
\hline
\end{tabular}

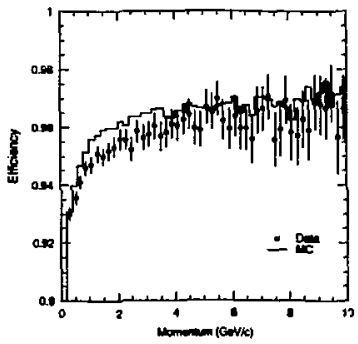

Figure 5.3: The efficiency for linking "good" CDC tracks (sec Chapter 7 for a defnition) to the VXD as a function of track momentum. The points are the measured efficiencies in the data the solid bistogran is the same quantity as determined in the MC simulation.

good agreersent here demonstrates that our understanding of the various resolutions and aligoment problems is approximntely correct. 

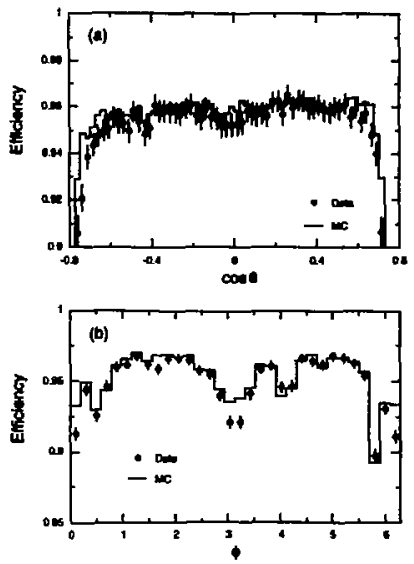

Figure 5.4: The efficiedcy for linting "good" CDC tracks (sec Chapter 7 for a definition) to the VXD a a function of (a) track polar angle and (b) track azimuthal angle. The points are the measured efficicncies in the data, the solid hirtogram is the same quantity as determined in the MC simulation. Note the effect of the $0.4 \mathrm{~cm}$ sbift of the VXD along the beam axis, which leaves the efficiency in $\cos \theta$ sowewhet esymmetric. 


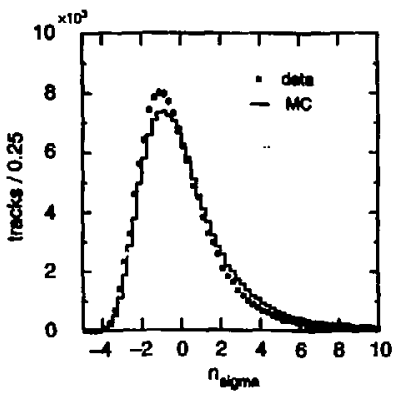

Figure 5.5: A comparison of the $\sqrt{2 \overline{x^{2}}}-\sqrt{2 \pi_{\text {a.o. }}-1}$ distribution for the CDC+VXD fit for data (points) and MIC (histogram) tracks.

As would be expected, adding VXD hits to tbe CDC track dramatically improves the resolution on nost of the track parameters over those measured by the $\mathrm{CDC}$ alone. The CDC+VXD results for these resolutions are also shown in Table 5.1. The momentum resolutions certuinly benefits from the addition of the precise VXD points clase to the IP; the combincd momentum resolution is $\sigma\left(p_{\perp}\right) / p_{t}^{2}=\sqrt{0.0026^{2}+\left(0.0095 / p_{\perp}\right)^{2}}$. Due to the staall kever arm of the VXD, the combined measurements are still affected by the precisiou of the CDC. In particular, tive single track impact parameter resolutions in the r. $z$ plane are noore than three timea worwe than those in the $x-y$ plane due to the lower precision of the CDC messurement of the track dip ande.

An estimate of the impact parameter resolutions for bover momentum tracke can be minde from the widthe of the distributions of doca $x_{x y}^{I P}$ and docarz by uving the MC to unfold the effects of heavy hadron decays and IP motion. The results of this study are shown in Figure 5.6 for tracks at $\theta=0^{\circ}$ and tracks at $|\theta|=70^{\circ}$. The impact parameter 
resolutions obtained for trecks with mowentum $1 \mathrm{GeV} / c$ are $\delta($ docanj,y) $)=70 \mu m$ and $\delta($ docalP $)=80 \mu \mathrm{m}$ at $\theta=\sigma^{\circ}$.
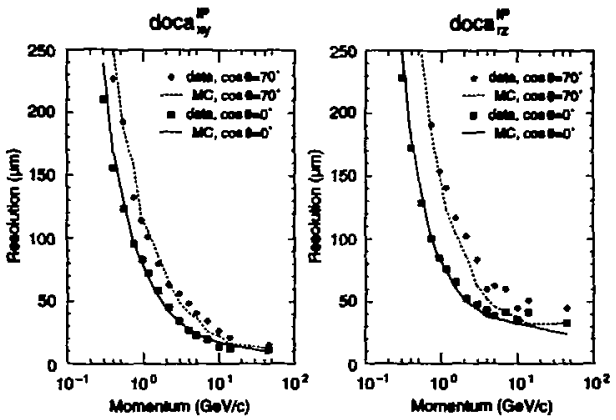

Figure 5.6: The impact parameter resolutions as a function of track moenentur and polar angle, for the $x-y$ and $r-z$ plares. The solid lines are tle resolutiona obtained from the MC; the points are those from the dats obtained by correcting the observed distributions for the presence of long-lived mesons. Two sets of reolutions are shown: thowe for tracks with normal incidence on the VXD ladders $\left(0^{\circ}\right)$ and thowe with a relatively large polar angle $\left(70^{\circ}\right)$. Note the worsening of the impact parameter resulution due to the extra inultiple scattering in the effectively thicker detector at large angles. 


\subsection{Determination of the \\ Primary Vertex (IP) Position}

The inclurion of this section breaks the flow of the discuesion of treckins performance, but is is neceseary to define what is meant by the event primary vertex or IP position with respect to which all track impact parameters are measured. Up to this point, we have used the term IP to mean bosely "where the $Z^{0}$ decay oceured". Here, we will make the distinction between the SLC IP, which is defined the center of the huminous region of the overlapping beans, and the event Primary Vertex (PV), which is where the tracks in the cvent appear to originate, given the information provided by the SLD tracking system.

\subsubsection{Transverse Position}

In principle, one could find the PV for each event from fitting all of the tracks which appear to come from a single, central point to a common vertox. This results in an error on the spatial position of the PV in the $x-y$ plane that looks like an ellipse due to the inevitable presence of collimated jets of perticles. This is shown schematically in Figure 5.7. Typically, the size of the crrors are $100 \mu \mathrm{m}$ along the ellipee major axis, and $15 \mu$ atong the ellipse minor axis. As these values are large compared to the resolutions discused above for the SLD tracking system, they would introduce unacceptably large errors on the track impact parameters if this were the only way to find the PV for each event.

Fortunately, the SLC luminous region is stable and extremely small. As the beams must actually collide to produce useful luminosity, the average position of the collision poimt is stable over times of at lexst one hour during smooth running. Steering correcturs in the SLC final focms driven ly feeciback loops make small corrections to the beam positions over this time to maximize the luminosity, but the amount of induced motion is small. So, rather than attenpt to find the PV event-by-event, we can subatit ute the average position of the SLC IP. In addition to drastically reducing the mapritude of the position error, averaging over events mitigates several other 
problens: the error ellipe on the mean IP will be roughly circular, removing the asymthetry induced by the jet axis; the bien on the PV position due to single tracks will be greutly reduced; and poseible correlations bet ween the PV position and those of decay verices in events with many secondary decayo will be ementially elimianted.

To find valuss for the average IP (IP) position over the entire run, the SLD date sarple is divided" into sets of $\mathbf{3 0}$ sequent ial hadronic $Z^{0}$ decays (events selected with loose criteria), except when a run ended more thm 20 events into a set, at which point the set is endedl. A trial (IP) is determined for eacls set by fitting for the PV, then averaging the result. The (IP) for each set is then derived by fitting all tracks which have VXD hits and which come within $3 \sigma$ of the trial (IP) to a common vertex. The fit (IP) is theu used as a new trial (IP), and the procksw is iterated until it converges. Typically, 330 tracks are used in a fit and the fit couverges after 5 itentions. The $x^{2} /$ dof for the fit and the fraction of tracks within $3 \sigma$ of tle fit (IP) are monitored for each set and compared with those fron previous and suberuent sets to identify sets which might span a major shift in $I P$ position. Information from the SLC correctors is used to help determine exsctly wen a major shift owers. When a major shift is found within a set, the boundaries of the set are changed to coincioie with where the IP shift occurs while still maintaining $\sim 30$ events per set slyenever poesible, and the fitting procedure is repeated. For a set to be used in the analysis, the $x^{2} /$ dof is required to be $<1.3$, and the number of tracks used in the fit is required to be more than eight times the number of events in the set. The fit (IP) pasition for the set in which an event resides is then used as the best estimate of the PV position in the I-y plane for that event.

The uncertainty in the $\langle\mathrm{P}\rangle\left(\sigma_{i p}\right)$ is the cumbination of the statistical error from the fit $(\sim 3 \mu \mathrm{m}) \bullet$, the extent of the SLC Iuminous rezion ( 1 $1 \mu \mathrm{m})$, and the motion of the IP within a set $(\sim 6 \mu \mathrm{m})$. This totals $\sim 7 \mu \mathrm{m}$ when added in quadrature. There

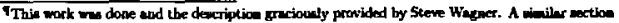
nppears In Ref. [182].

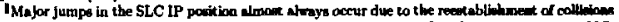

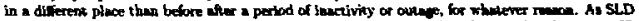

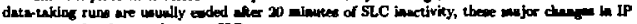
poaltos end w happen between SLD rurs.

"Coropere this to 15 wir for the single exen PV determiantion, sbove.
} 


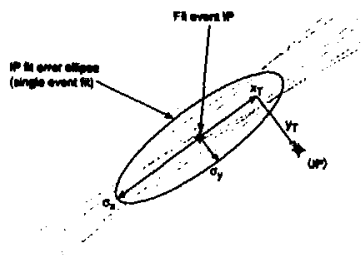

Figure 5.7: A diggran stowing tive definition of the rariables used in the discumion of tlee $\langle I P\rangle$ fit quality. Shosn are the possible results of fitting the IP position for a given event, with the error ellipe of this fit (which bes majox (minor) nxis length $\sigma_{x}$ $\left.(\text { sigma })_{4}\right)$. Also shown is the mean IP position (IP) for this of runs, and the two corrdinates specifying its location relative to the fit event. IP. The $(I P)$ position is $I_{T}$ away from the fit event IP along the major axis of the error ellipee, and $y_{T}$ elong the uninor axis.

are scueral ways to estimate $\sigma_{f F}$ wing the data. The distribution of track linpact parancters with respect to (IP) it $Z^{0} \rightarrow \mu^{+} \mu^{-}$events is shows in Figure 5.8. As $Z^{0} \rightarrow \mu^{+} \mu^{-}$events are not used in auy wry for the ditermination of (IP), they can provide an independent check on the precision and arcuracy of the beam poaition measuremeut. The $\sigma$ of the distributicn is $12.7 \mu$ s; when the trark extrapolation error is subtracted in quadrature, this gives $a_{j p}=6.7 \mu \mathrm{m}$, which confirms that our entimates of the uncertainties in extracting the (IP) are approximately correct. In addition, in hadronic events where most tracks fit to a common vertex, the distance $\left(y_{r}\right)$ between the (IP) and the fit vertex, projected onto the minor uxis of the fit vertex error ellipar, aleo contains informstion on $\sigma_{s p}$.

The quantity $y_{T}$ is calculated for each eveut using the fitted primary vertex for that event. The primary vertex for each event is found by beginning with the four tracks whose inpact parameters with reppect to the (IP) are amullest. Tracks which make the $x^{2}$ vertex probability the largert are added until the overall $x^{2}$ probability drups below $1 \%$. The poeition of the event PV and the error ellipse perameters ace 


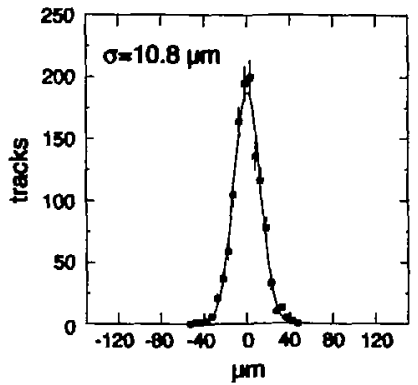

Figure 5.8: The impect perameters of muons from $Z^{0} \rightarrow \mu^{+} \mu^{-}$events to the (IP). As they are not used in the determination of the (IP) position, thene tracis provide an independent measure of the resolution on the $\langle$ IP $\rangle$ position.

then calculated. Figure 5.9 shows the distribution of $y_{T}$ for the evejus wlyere

- $\geq 7$ tracks are included in the primary vertex

- $\geq 70 \%$ of the tracks in the event are usidd in the primary vertex fit

- The error on $y_{\tau}$ is lets than $15 \mu \mathrm{m}$

- the overall $x^{2}$ vertex jrolmbility is greatcr thas $1 \%$.

The data points are overlyed on a MC simulation in which $7 \mu \mathrm{m}$ of IP motion has been addad. The non-gaussien tails are similar in both MIC and date. This distribution for all events, while having high ntatistics, includen sorne contamination from evenus contsining heavy quark decays, which couk bias the fit PV position. We can produce another sarople by requiring 


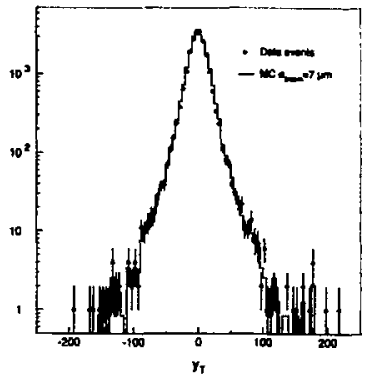

Figure 5.9: The $y$ distribution for all events sistisfying moderate vertexing critetia (see text). The points are the date, the solid bistogram is the MC with an input. smearing of $7 \mu \mathrm{n}$ in the (IP) position. Small taiks can be Been on both dirtributions.

- $100 \%$ of tracks with VXD bits are ued in the primary vertex

- the overall $x^{2}$ vertex probability is greater than $10 \%$.

These events contuin a very pure sample of light quark events, but the resulting $y_{r}$ distribution is lower in statistics, as shown in Figure 5.10. Nonethelewe, the $y_{T}$ distributions for both these samples were consideted; all distributions ogree with $\sigma_{i r}=7 \mu \mathrm{m} \pm 2 \mu \mathrm{m}$ for the $1993 \mathrm{deta}$.

Even though great care has been tuken to minimize the potestial for large beam motion within a set of events, it is gtill possible that some sets contain events which occur a large distance away from the (IP). This would lead to large numbers of tracks in each of these events that mise the (IP) by a significant amount, which would lead to a much ligher probability for these events to be taged af $b$ quark evente. This would effect any aralysis tinat attempts to ue the precision tracking a $n$ flevor tag. 


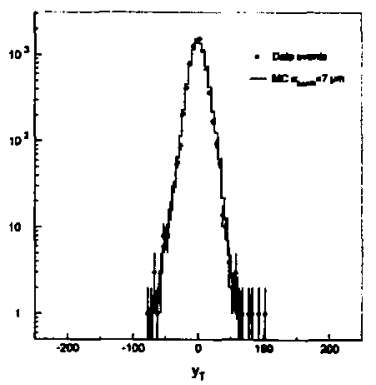

Figure 5.10: The $y \tau$ distribution for events with all tracks in the primary vertex (me text). The points are the date, the solid histogram is the MC witb en input smearing of $7 \mu \mathrm{m}$ in the 〈IP〉 pucition.

In an attempt to set an upper limit on how often this could happen, the ame distributions which are used to cotimnte $\sigma_{i f}$ sre searched for evidence of nod-zaunien toils. The $\mu^{+} \mu^{-}$inpact peremeter distribution show's no evidence for pon-gauden tails, but the statistics of this sample are not lerge enough to rule out non-gauesian tails in the much larger sample of leadronic everty. The highest statistics check, the $y_{r}$ distribution in all hadronic cvents, shows sinitar smull son-gausian tails in both the $M C$ and data. The $M C$ indicates that this is cauned by the occusional inclunion of $B$ or $D$ decey tracks in the vertex fit, which then pulis the PV vertex pocition sllphtly. All other distributions show straller gon-gamais trils than this one. We take the $y_{s}$ distribution in all badronic events as a conservative linit on the dae of these tails, which keads us to include a secosd IP extent $\left(\sigma_{\lambda p}^{\prime}\right)$ of $100 \mu \mathrm{m}$ is $<0.25 \%$ of the data to sitnulate this effect. The full shilt in the results obtrined with and witbout this additional tail will be taken as the systematic error due to thin ofiect. 


\subsubsection{Longtudinal Position}

Since the SLC buncles are apruximately $1 \mathrm{~mm}$ long, the luminous region along the - $\quad z$ aris has auch larger phyical extent than in the $x-y$ planett. There in nothing to be gined by averaging many events together in this plate to obtain the maen IP pocition, since the tracking resolution is much finer then the poesible distribution of interactions. The bese estimate of the PV $z$ poition for each event comea from a technique using only the median $z$ of the tracke in the evest jineif. Each trecte with asocisted VXD hits is extrepolated to the point of clasen approach to the (IP) in the $I-v$ plase, and the $z$ cooxdinete of the track at thl point in denoted as at. A selection of tracis is then wade $w$ require the track $x-y$ impact prameter to be leas than $500 \mu m$ and the trect to pans within $3 \sigma$ of the (IP) beed on the estimated treck impact parameter error and $\langle I P\rangle$ error. The evert IP $z$ locstion is simply defined as the modian of the 2 dow values from the selected treclu. For the amall frection of events with no tracks passing this rekection, all tracks with VXD hits are wed. The thoice of the median z metlod invead of the more common approuch involvins: vertexing is besed on the result of a MC study alowing that the median $z$ is more robust neninst the biases due to the inclusion of tractes not orginating from PV. The typical resolution for locating the PV $z$ as derived from MC are $(32,36,52) \mu m$ for (uds, c,b) exents respectively. The tails of the PV $z$ residual distributions can be charucterized by tlie frection of events with residual $>100 \mu \mathrm{m}$. The fraction of such events are $(0.8 \%, 1.6 \%, 7.5 \%)$ for (uds, $c, b)$ events according to the MC simulation.

\subsection{The Impact Parameter Distribution}

The final elcment in deacribing the performance of the SLD tracking syctem is aloo the mast relcvant. Shown in Figure 5.11 is the distribution of the normalized inpect parnmeters doca $a_{x y}^{I P} / \delta\left(\operatorname{doca}_{s y}^{I P}\right)$. The agreement between date and MC is quite good, even out to $20 \sigma$ on the tails. The wgremernent is equally good in tbe $r-z$ plane. The

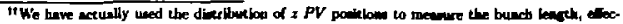

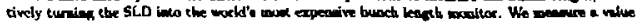
conivient with the expectution of $1 \mathrm{me}$.
} 


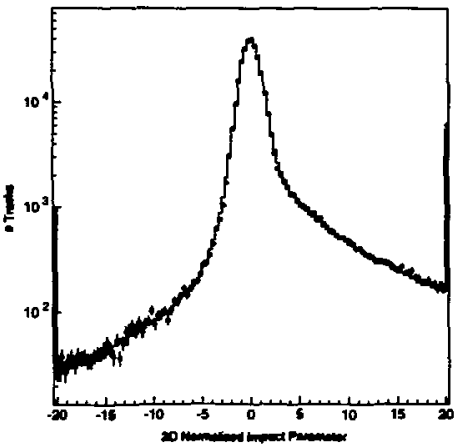

Figure 5.11: A comperison of the distributions of nurmulized impact peranetern in the $x-y$ plane for all hadronic events in the datu (points) and MC (hietrogram). Tho tracks included here are "good" trucks (see Chapter 7). The under- and overflow bin are also shown.

only "smwaring" which hes betn added to the MC simulation is the residual alignmene errors of the VXD and the CDC interisal and global allogments. The bocal aligament smearing was done on a CCD-by-CCD, ladder-by-ladder, and cell-by-cell bari in order to get the $\chi^{2}$ distributions to match between data and MC. An averaso anoune of smearing wrus added to each element, and at no time was there any tuming done on the tails of the distributions in Fig. 5.11 to stcenst to make the Leila agree. In thin version of the MC, the origial gues of the CCD alignment position precision was underestimated, and there was in addition an error in the almulation, both of which coneplred to meke the core of these dintributions wider in MC than in data. Give the "true" track parameters from MC, the diarribution were sctully mumenes to 


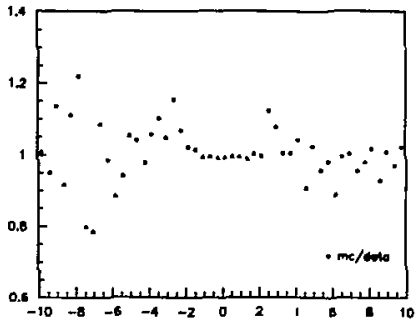

Figure 5.12: The ratio of the distributione of the normalized impect puranetur for the unaracared MC and daca (see text)

remove this effect. The ratio of ungmuared MC to the data is sbonn in Figure 5.12; the unamearing correction was ofly applied in the "cone" region, and ito magnitude we calculated solely fron the MC. The agreement betwen MC and deca in the core region reflects the true quality of the MC simulation, not a conspiracy to force agrement between the simulation and the dats. The full change in the results of the amelysis by applying this correction will be taken as a syetematic erroc.

In the following sections, we discuso anpects of the tracting gystem performante that are relative to the reconstruction and isolation of events containing $D$ mesons for the charm usymmetry memsurement. Two methods are used to find Ds: one requires

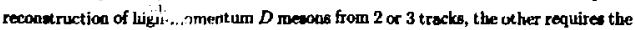
wolation of the $D$ decay vertex. T!'"1, the proper simulation of the mans resolution of the detector and its vertexing capabilitit are neceasary in order for us to have confidence in the MC jesults. It is worth noting that $\mathrm{w}$. "rin concerned here ebout the traching performance es applied to uniti-track combiustions as oppoed to the single variable distributions we have been diecussing. 


\subsection{Mase Resolution}

To study the mes resolution of the SLD tratisg systers under conditions anilar to those encoupleted in reconstructing $D$ mewons, we can examine high-mosentum $K^{0}$, and $\Lambda$ decays (collectively called $V^{\bullet}$ deswy), and compare the width of the mass penks in data and MC. If the tracking performance in properly inoulated a bigh momeut um, we ahould obtain reasonable agreement betwen the dace and MC distributions. Candidates for $K^{0}$, and $\Lambda$ decayn are found by pairing all conbinations of oppositely-aigned tracks and ascigning either both tracts the $\pi$ moes $\left(K_{i}^{*}\right)$ or one the proton mave and one the $x$ mas. At high monentum, the ferter of the two particles in $\Lambda$ decay in aluost always the proun; wing this information for the moo assignments reduces the combinatoric backgroutsd. Several cuts are applied to anch $V^{\circ}$ candidate to obtuin a clean sarpie[176]. The pair of trecke forming the candidate $V^{\circ}$ must pass within $2.5 \mathrm{~mm}$ of cach other, and their actual dimance of cloeser approack must be leas than $3 \sigma$ of the uncertainty on their comanon point of ocigin in the plane perpendicular to the sum of their momente. The difference between the $V^{0}$ candidate momentum direction and iLa flight direction muat be ben than $1^{\circ}$ in the $x-y$ plare, and leas than $2^{\circ}$ overall. Figures 5.13 and b ahow the iavariane menes of the $K^{\circ}$ and $\Lambda$ candidates for $x_{\gamma}>\mathbf{~} \mathbf{0 . 2}$, which is the lower of the two momentum cuts that are used in the $D$ meson reconstruction analyois. There is resonable agreement between the data and MC simulations, which gives us some coofidence that we will be able to reconstruct accurately tbe manses of $D^{0}$ and $D^{+}$mesoas for the cherm aytnotry analysis.

\subsection{Vertex Finding}

There are two concerns about tracking performance that are relevent to an andyis that depends on the isalation of a decay vertex involving two or mori tracks. The first is whether or not suxch a vertex can be found, including whether or not the tracks are propety reconeructed, which is enentially a trecking (vertexing) scioscy queution. The econd is whether ar not, given that $n$ vertex in found, the vertax can 

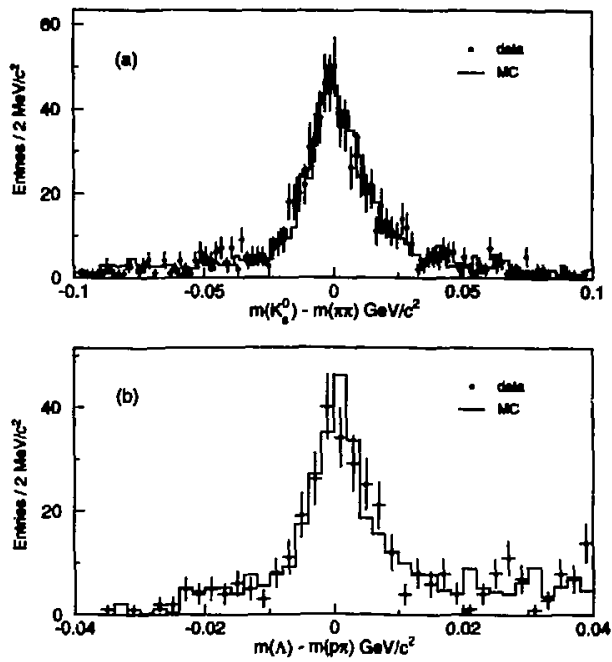

Figure 5.13: The invariant mass distributions for (a) $K^{0}$ and (b) $\Lambda$ candidetes with a total nomentum greater thas $9 \mathrm{GeV} / \mathrm{c}\left(\mathrm{x}_{v}>0.2\right)$. The points (himogran) reprewent the data (MC) distribution. A reaconzble agreenent between the duse and the simulation cen be ...... 


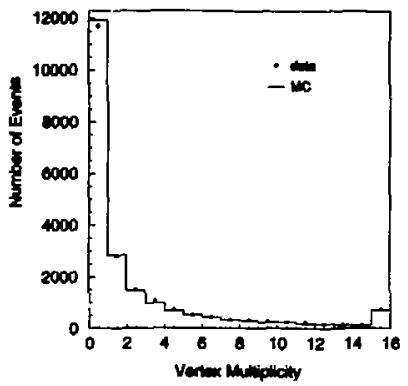

Figure 5.14: The distribution of the number of two-track sertices found in hedronic ovents, comparing the data (points) and MC nimulation (histograin).

be isolated frou the other vertices around it which arise from rasdom cumbination of unasociated tracks, which is a vertex resolution quest iont. We will discius each of these concerns in turn.

Ratber than give an explicic exposition of the vertexilu efficiency as $r$, function of vertex monenturn and vertex decay kength, we present berc evidenice that both the tracking efficiency and the track resolution is jroperly silusuluted in the MC*. To do thls, we can conpare the data and MC distrilnutions of the number of twotrack vertices found in all hadronic events. We must muke sonke cut to conider only those vertices wi_ch are some distance from the IP; this we choose to be 30 . Thit distribution then contains the efficiencies for trusk fitsling and cocrect track reconstruction, as wall as a clieck that the crrory oll the wertex fit are calculaced cocrectly. The tracks usert in making this plot are "guod" trucke as defined in Chapter

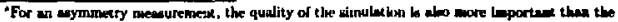
actud punuercal vilue of the vertexins ctickikicy.
} 


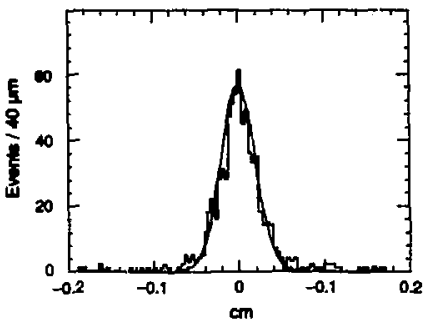

Figure 5.15: The fligdit distance rosolution of the SLD tracking system as nimulated in MC for $D^{+} \rightarrow K^{-} \pi^{+} \pi^{+}$decays. This fit curve is a single gaussian of width 200 $\mu \mathrm{m}$.

7. The wertex fit is performed using the SLD wriexing routines zxTwo followed by ZXFIT, which perform a 1-C fit for the vertex proition and crror matrix. The momenta of the trarks are reevaluated at the vertex pouition. Figure 5.14 shows the number of vertices with flight distances greater than $3 a$ for all (selected) hedronic events in tbe 1993 daes semple and the correspouding MC simulation. Good agreement is meen betwen the two distributions, wlich it lies that the track- and vertex-finding eficiencies ure well simulesed in the MC.

The resolution of the tracking system in mennuring decay leugths can be eotimated from the MC simulation, as we bave no way of knowing the exact decsy lengths of secondary vertices in the dats ${ }^{+}$We huve used the MC to eatimate the decsy length rewolution for $D^{D} \rightarrow K^{-} x^{+}$and $D^{+} \rightarrow K^{-} x^{+} \pi^{+}$decays, as these two and thre-track modes are those uned in the analysis. The MC nistributlon of the mearured minus the true decay length for the $D^{+}$decay is shown in Fizure 5.15. The width of the gingle

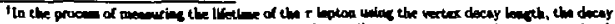

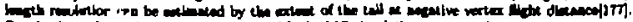

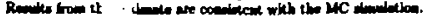


gausoien fit is $200 \mu \mathrm{m}$. Since the menn $D^{0}\left(D^{+}\right)$decay length is approximetely 1.5 $\mathrm{mm}$ (2.5 mun), requiring found vertices to be separated from tbe IP by 30 of so will still renult in an efficient vertex tas while supprevalug the large gumber of randow vertices the oair from tracks peusing close to the IP. 


\section{Chapter 6}

\section{Hadronic Event Selection}

One of the advantages of doing plysics in the envirament of an $e^{+} e^{-}$pollider is that orie expects the backgraund processes that could mitric an event of phyaics interent to occur infrequently enogh that thr trigger algorithm that cansce events to be recorded can be relatively simple and indiscriminant, and yet not be overwhelmed by a flood of date. This approsch also leads to a very efficlent tribger arorithen, where the efficiency for actually; recording the events of interest is for all prectical purponea $100 \%$. In orler to be this efficient, however, the tricjer aigorithm must be made sufficiently indiwcriminant, which results in eanentinlly all events that reginter any a onable amount of energy in the detector being written to tape. Nonetheles, this „proach has worked well, even at the SLC, where accelerator-induced backgrounds can be more of $n$ problem that at a typical storage ring collider. Since magpetic tupe is a relatively cheap storage medium we can afford to write to tape a data stream which contains less thun $1 \% Z^{0}$ events and sift through later to select out the decays that we want for physico analysis purposes. Thio chapter describes in some detall the selection criterie applied to the raw events to pass the trigger, the initial $Z^{n}$ selection filter, and our definitions of a hadronic $Z^{0}$ decay. 


\subsection{The SLD Trigger}

The trigger criteris are dencribed in detall in Ref [178], and will be anmariand briefly here for completence. During the 1993 SLD run, thete were aix main clenes of triggers: a total enercy trizger, a charged track trieger, a "hedron" (HAD) trimer,

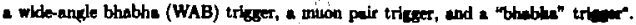
The energy trigger required a minimum total energy of $8 \mathrm{GeV}$ ', where the mam in then only over thowe EM (HAD) calorimeter towers containins more then 60 ADC connts (120 ADC counts) of deposited energy, which corresponds to an eneros threiloild of $245 \mathrm{MeV}(1.296 \mathrm{GeV})$ per tomer. Only the calorimeter rysteme were read out when this trigger fired. The charged track trigser was baed on a pattern mep of the celle that migkt be bil a a charged track of momentum greater then $250 \mathrm{MeV} / \mathrm{c}$ paned through the CDC[179]. A hit cell was defined as a cell where pulses consistent with hits from tracks fired a discriminator on 6 of the poenible 8 wires. Those cventa contnining two tracks pascing through at least 9 superlayers of the CDC and lying roughly $120^{\circ}$ apart were paned by this trigser, and al of SLD was read out if it fired. The HAD trigger is a combination of tbe previous two trigsers, as it required oos charged hinck of 9 or more superlayers and a large energy deposition in the L.AC. As it was expected that most $Z^{\prime \prime}$ events woukl entinfy this trigser, the entirety of SLD wes reat out when this trigger was satiafient. The WAB trigger wo designed to isure that all wide-angle $e^{+} e^{-}$peirs were recorihd, even those at anglos where the track gtubs in the $\mathrm{CDC}$ were not long enough to satisfy the track trigger requirements. Thin trigger also initiated readout of all of SLD. The mison trigger required a combination of $\mathrm{s}$ charged track in the CDC and hits In opposite WIC octants, as this would be

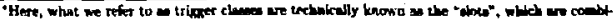

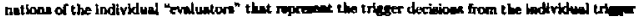
procemors in the dele acyilisition syite.

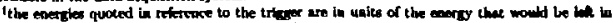

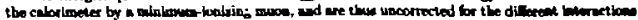

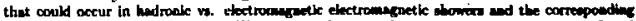

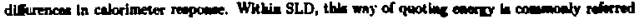

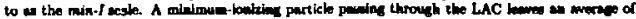

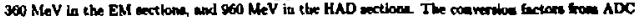
counts to energy are $4.1 \mathrm{MleV} / A D C$ cound (10.8 MeV/ADC coupt) for tho EM (HAD) hyest of the calorimeter.
} 
the aignature for a $Z^{0} \rightarrow \mu^{+} \mu^{-}$event. Once agaln, all of SLD was read out If this trigge wa satlefied. The bhabha trigger required a total energy (EM scale) in the EM2 section of the LUM to be above $12.5 \mathrm{GeV}$ in both the nurth and south detectors, where the sum is mede over toners with tnore than $1.25 \mathrm{GeV}$ of energy. This energy deponition is what one might expect as a slenature for an $c^{+} e^{-}$pair above some splash of beckground. This ouly trisgered the resdout of the LUM/MASiC system, and thus will not concern us further.

\subsubsection{The Tracking Trigger Veto}

A number of vetos are added to the trigger system in order to prevent excesive detector dead-time caused by processing huge events where the detector is sprayed with accelerator-induced lackgrounda. Acceletator hiccups could happen in many different ways (sec Ippendix C), but the effect on SLD is more or less independent of the cause, as the detector will end up completely blasted with noise. One of these vetos, the one employed in the track trigger, has a large impect on this analysto. The tracking trigger ret 7 causes an event with wore than 275 cells having 6 out of 8 wires hit to not be read out. In the nomind configuration, this has a reiatively srabl effect on the number of $Z^{0}$ decays that lack tracking information. Unfortunately, during approximately two-fiftio of the $1993 \mathrm{run}$, a hardware problem effectively shited the number of hit cells up from a minimal value of 50 up to a mininum value of $100^{7}$. The effect of this is that in approximately $12 \%$ of hadronic events the CDC information is missing when it would ordinarily be prcsent. For the $\alpha_{4}$ analysis, this could have severe consequedces, as it is easy to see that the veto preferentially renoves 3-jet events, as they tead to have higher charged multiplicity and would hit more celis. In particular, since $b$ events have a higher average multiplicity, 3 -jet $b$ events are vetoed more often than 3-jet events from other quark flavurs. All of these cfiects could potentially bias the flavor-independence test if they were to be ignored". A simulation of the track trieger veto does in fact exint, however, snd cas be used on the MC to provide the

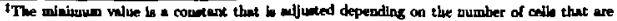
loppermive at a given time.

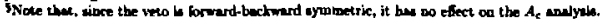


efficiencies for trigger and event selectlon for the given quark flavore ard event jet multiplicitio that ghould match tbe data. This has been diseused in greal detail in Ref. [166]. Variations of the rate at which ovents are vetoed will be considered below as a systematic error.

\subsection{The Hadronic Event Filter}

The initial event selectlon is done using calorimctry infortnation only, as it is much faster to process compared with the tracking data. The filter glgorithm used at this stage, called "EIT pass-1" [178], requires that the events arr telatively spherical axd have good forward-backward noment un lalance. It is baned on thee LAC quantities:

- NEAIHI, the aumber of LAC EMI towers with sigals gbore $60 \mathrm{ADC}$ counts ( $250 \mathrm{MeV}$ min-I)

- EHI, the sum of the energy deposited in all EM (HAD) tomers with signals greater than high thresholds of 60 (120) ADC counts. (This is equivalent to 250 $\mathrm{MeV}(1.3 \mathrm{GeV})$ min-I.)

- ELO, the sum of the energy deposited in all EM (HAD) towers with signals greater than low thresholds of 8 (12) ADC counts. (This is equivatent to 33 $\mathrm{MeV}(130 \mathrm{GeV})$ min-1.)

The filter requires that each event satisfy:

1. ${ }^{\prime} E M H I \geq 10$

2. $E H I>15 \mathrm{GeV}$ min-I

3. $E L O<140 \mathrm{GeV}$ min-1

4. $2 \times E H I>3 \times(E L 0.70)$

3. The north and south bemispheres of the detector must each have NEMHI $>0$ 
Cuts 3 and 5 tend to renuve beam-wall events and other beam burp events; cut 4 removes thoee events with large numbers of SLC muons (see Appendix C) pasing through the LAC. The coubined efficiency for a hadronic $Z^{0}$ decay to pass the trigger and the EIT pass-1 filter is npproximately $92 \%[180]$.

\subsection{Hadronic Event Selection Cuts}

After applying the EIT pass-1 filter, 63553 events remain in the data sample from the 1993 mu. In order to redice this to a sample of hadronic events useful for physics andysis, ne apply cuts desigual to select exents contsined within the fiducial region of the SLD detector. Since we use charged trucks as a basis for unuch of the analyals, this restricts us to the barrel region of the SLD, as the CDC starts to lose trecking efficiency outside of $|\cos \theta|>0.8$. We also wish the energy thew of the event to be well contained tithin this fiducial region so as to guarantee that we are not missing large portions of the event that have ended up in the detector enckaps. In addition, we should note that, since it poesesses the simplest geometry, the barrel region of the detector is also the easiest to model in the MC, and can be considered well understood ${ }^{\prime}$ in this context jelative to the rest of the SLD. Since number of the cuts are based on charged tracks, we first set fortll a definition of a "charged track", which is any track satisfying:

- transwerse monenturn relative to the beam axis $p_{\perp}>200 \mathrm{MeV} / \mathrm{c}^{2}$

- $|\cos \theta|<0.8$

- distance of ciosest approach in the $x y$ plane $\left(\right.$ doca $\left._{x y}^{I P}\right)<5 \mathrm{~cm}$

- distnnce of closest approach in the $r z$ plane $\left(\right.$ doca $_{r z}^{(P)}<10 \mathrm{~cm}$

Since slightly different event selection criterio were used for the two analyses presented here, we prosent two descriptions of the eveat selection cuts.

TThe $A_{c}$ nalysis used a uinitarg trancerse momentum of $p_{\perp}>200 \mathrm{MrV} / \mathrm{c}$ 


\subsubsection{Event Selection for the $\alpha_{d}$ Analyais}

The sclocted events for the flavor indepentence analysis are required to satisfy:

-7 charged tracks

- $\left|\cos \theta_{\text {Thrme }}\right|<0.71$

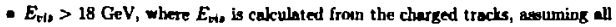
tracks to be pions.

One can see that these cuts meet the requirements uentioned above for defining a set of fiducial bedronic events with minimal background from ot her processes.

The one exception to the exclusive use of charged-track information in this analysis is the determination of the thrust axis direction, where we uoe the thrust axis calculuted from calorimeter clusters. The reason for preferring the calorimeter determination of the thrust direction is shown in Figure 6.1, where the thrust direction calculated for each event from calorimeter atnl track information is plotted. One can clearly see the cases at high calorimeter $\cos \theta_{\text {Thrat }}$ where the tracking determimation falls because of lost information. Since one expects the global esent properties of these events to be nodeled kos well than those where many tracks are present in the fiducial volume, it is best to exelude theru from the analysis sample.

Figures 6.2 show the data sample before cuts, with the hadronic event MC overlayed. After applying these cuts, 28036 events remain. Table 6.1 shows the efficiency for the different event flavors to pass these selection crits for the period prior to the trigger veto problem (henceforth called the "Pre-Veto" period), the period of running during which the track trigger veto was incorrectly set (the "Veto" period), and for the later run time after it had been fixed (the "Non-Veto" period). Any small flevor bias in event selection will be explicitly zorrected in the analysis, but, as can be ween, any eveat selection bias is staall. The veto period does show some bies for rejecting $b$ events. This is expected, as they tcud to have a higber charged multiplicity, and thus sbould trigger the veto more often. The surviving backgrounds are small and are neglected in the analyss. 
It in intereating to break this domn further Into the efficiencies for 2- and 3-jet ovents to paso the selection culs (und the trigger veto). Table 6.2 containg morc dotailed cut information. The effect of the tracking trigger veto is readily seen, as the eticiencies for 3-jet events to paes the veto and cuts are from $7 \%$ to $12.5 \%$ lower during the Veto perbod compared with the rest of the run.

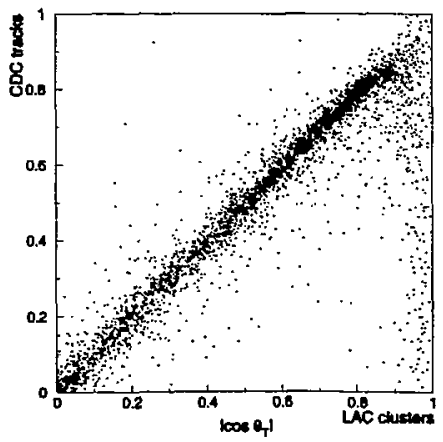

Figure 6.1: A comperison of the direction of the thrun axis as determined by the CDC (tracks) and the LAC (calorimeter clusters). The effects of the linited oolid angle coverage of the CDC are rendily apparent, as there are many events where the majotity of the evergy is deposited in the LAC endcaps (and hence at large $\cos \theta$ ) in which the thrust axis direction L miecalculated by using the CDC-only information. 

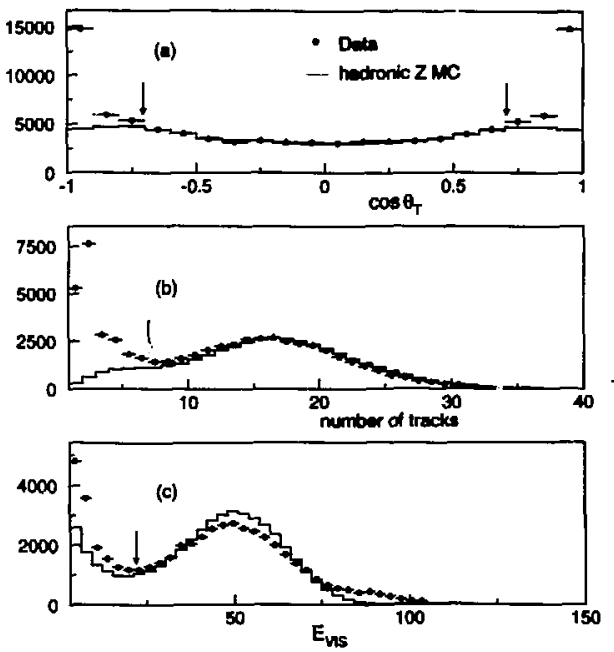

Figure 6.2: Distributions of the event selection variebles. To tnrke each plot, all of the other cuts bave been applied except for the one being plotted. The arroms indicate the values of the cut on exch of the variables. 
Tabte 6.1: The efficiencies for bedrunic events of different primary quark flavon to pas the event selection cuts.

\begin{tabular}{|c|c|c|c|}
\hline Run Period & wds efficiency & ceficiency & b efficiency \\
\hline Pre-Veto Period & $0.5749 \pm 0.0020$ & $0.5738 \pm 0.0037$ & $0.6594 \pm 0.0033$ \\
Veto Period & $0.5457 \pm 0.0017$ & $0.5392 \pm 0.0033$ & $0.5115 \pm 0.0029$ \\
Non-Veto Period & $0.5934 \pm 0.0029$ & $0.5969 \pm 0.0055$ & $0.5926 \pm 0.0049$ \\
\hline
\end{tabular}

Table 6.2: The efficiencies for hadronic events of different primary quark flavors and different numbers of jets to pass the event selection cuts. The $\mathbf{E}$ algorithm with a $y_{\mathrm{N}}$ vilue of 0.8 was used to obtain these values.

\begin{tabular}{|c|c|c|c|}
\hline Run Feriod & uds eficiency & c efficiency & b efficiency \\
\hline Pre-Veto Petlod: & & & \\
2-jet ercnts & $0.6145 \pm 0.0023$ & $0.6177 \pm 0.0043$ & $0.6113 \pm 0.0038$ \\
3-jet events & $0.4619 \pm 0.0039$ & $0.4534 \pm 0.0073$ & $0.4512 \pm 0.0065$ \\
Veto Period: & & & \\
2-jet events & $0.6013 \pm 0.0020$ & $0.5979 \pm 0.0037$ & $0.5751 \pm 0.0033$ \\
3-jet eveuts & $0.3868 \pm 0.0033$ & $0.3776 \pm 0.0061$ & $0.3299 \pm 0.0053$ \\
Non-Veto Period: & & & \\
2-jet events & $\mathbf{0 . 6 2 8 3} \pm 0.0033$ & $0.6329 \pm 0.0063$ & $0.6354 \pm 0.0056$ \\
3-jet events & $\mathbf{0 . 4 9 1 5} \pm 0.0058$ & $0.4964 \pm 0.0109$ & $0.4753 \pm 0.0097$ \\
\hline
\end{tabular}

\subsubsection{Event Selection for the $A_{c}$ Analysis}

Since, as will be seen, the $A_{c}$ aralysis is driven to obtain the largest posible sample of $D$ dccays, we can loosen the eient selection cuts substantially. We are not concerned sbout the energy flow of the event, wh the reconstructed $D$ meson direction is used instead of a jet or thrust axis to approximate the initial quark direction. Since the asyumetry is also largest at large $[\cos \theta]$, we would also like to be able to reconstruct as wany $D$ 's at large angles as is possible given the acceptance of the vertex detector. The requirement that thre tracks form a $D^{*}$ or $D^{+}$also tends to suppress beckgrounds from misneasured tracks, so that any degradation in the tracking performance at largo 
$|\cos \theta|$ atnounts only to an overall efficiency lose. To this end, we define the hadronic sample for the $A_{c}$ analysis to be those events which hase

- 5 or more charged tracks

- $\left|\cos \theta_{\text {Thrual }}\right|<0.8$

- $E_{\text {vis }}>18 \mathrm{GeV}$.

We use charged tracks in all cases, even to find the thrust axis directionl. Theos looser cuts result in an event saraple of 33,524 hadronic decays.

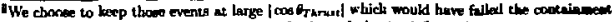
cut had the thrue exis direction been calculeted wies caborieneter informetiog. 


\section{Fart I}

A Test of the Flavor Independence of the Strong Interaction 


\section{Chapter 7}

\section{Flavor Tagging}

As mentiond in the introduction, we have chocen to use the differences in lifetime and decay saultiplicity among the different hadronic species a a tool to separate events contuining differcut flavoss of primary quarks. In principle, the tas abould be relatively insensitive to the kinematios of the tauged event (whether it had 2 or 3 jets, in particular), should be efficient, and should provide meximal purity foe all of the tagged semples. Simplicity can also be considered a virtue in this particular case. These criteris rule out the more complicated taps mentiosed in Section 3.4.1, as well as attempts to select heavy favor events by the exclusive reconstruction of charm or bottom mesons. As will be described betow, a remonuble compromine is to look only at the number of tracks in each event that mis the IP by a significant distanre, as this efficiently separates high-purity samples of $b$ and uds events, as well as a sumple of enriched purity. This chapter presents a detalled explenation ox this tagging method.

\subsection{Selection of Quality Tracks}

As will $i=$ described in Chapter 9 , this analysis depends in a critical manner upon our ability to understand the performance of the SLD tracking systern in as rauch detail as possible. In particular, the entire process of flavor separation require the MC to tell us the efficiencies for tagging an event of a given quark flavor for ench 
of the lags. As the tags are based on track multiplicity, it is imperative that the ensemble of tracks used in the tags are xell understood and well measured. To this end, we need to apply further cuts on the charged tracks used it he andysis so as to guarane that they are well-described by the NC simulation and do not heve some parameter or other lying way out on a mysterious tail of some du:tribution. These cuts hase the other beneficial effect of removing some tracks from light quark events that carny lifetime information, such as those from $K^{\circ}$ dexays, which can cont ribute to the light quark contamination of the heavy Havor samples. Tracks considered as -Tagging T. reks" must have:

- at least one VXO hit

- at least $40 \mathrm{CDC}$ hits, with the first hit at a radius less then $39 \mathrm{~cm}$

- a coinbined CDC+VXD fit quality $\sqrt{2 x^{2}}-\sqrt{2 \pi_{\text {d.a. }}-\mathrm{I}}<8.0$

- total momentum greater than $0.5 \mathrm{GeV} / \mathrm{c}$

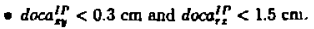

- an crror on doca ${ }_{x y}^{I P}$ of $<250 \mu \mathrm{m}$

The cuts are placed on tbe track parameters calculated frotn the combined CDC+VXD fit. In addition, tracks from condidate $\boldsymbol{K}^{\circ}$ and $\boldsymbol{\Lambda}$ decays and $\gamma$-cunversions found by kinematie reconstruction of tho-track vertices are removed from this analysis|181] to eliminate tracks from uds events that secun to come from particles with long lifetimes. All of these cuts tend to discard poorly measured or mis-recont ructed tracks, since they tend to have larger extrapolation errors, fewer hits, and larger IP miss distances than properly reconstructed, well-mcasured tracks. Figure 7.1 shows the dist ributions to which the cuts are applied, an well as the cut values, for the three classes of tracks containing different sources of bfetine information. Immediately following, Figure 7.2 shows the distributions of these sune variables for $\mathbf{M C}$ and data tracks, with all cuts applied except the one being examined. Within the cut values, the shspes of these dist ributions are well-modeled by the MC. 

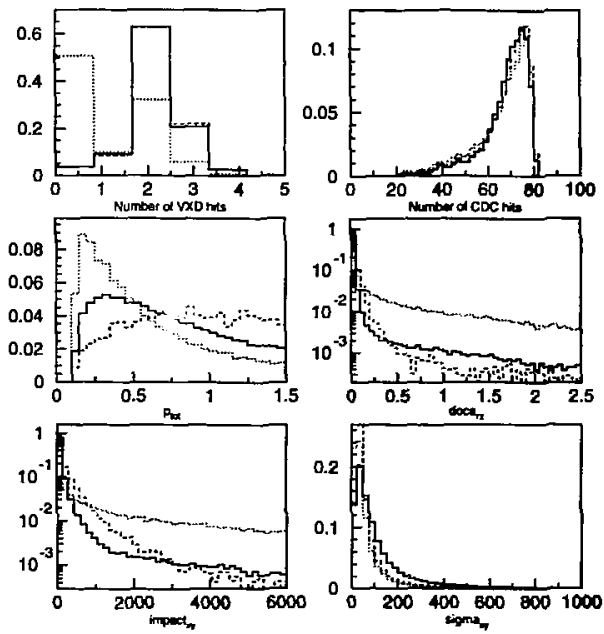

Figure 7.1: Distributions oi six of the variables upon which cuts are placed to select quality tracks. The curves shown sre solid for those tracks from uds events and/cr the fragmentation tracks in $c$ and $b$ cwnts (uds $-f$ rag), dotted for those from $\boldsymbol{K}^{0}$ and $A$ decays and $\gamma$-conversions (Vees), and dashed for those from $c$ and $b$ badron decays $(c+b)$. Some salient features are the barder momentum spectrum for those trecks from heuvy quark decays and the !ung tails on the irnpact parameters for strange derays and other Vees. 

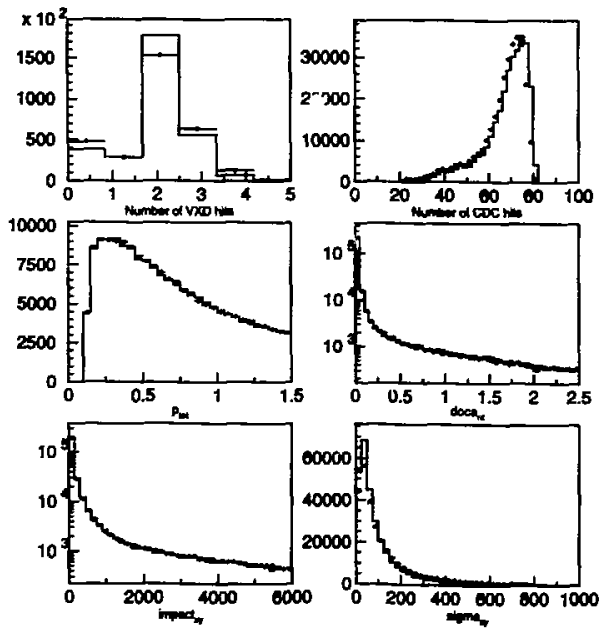

Figure 7.2: Dintributions of aix of the variables upon which cuts are piaced to select quafity track, compering data (points) and MC (bintogram). 


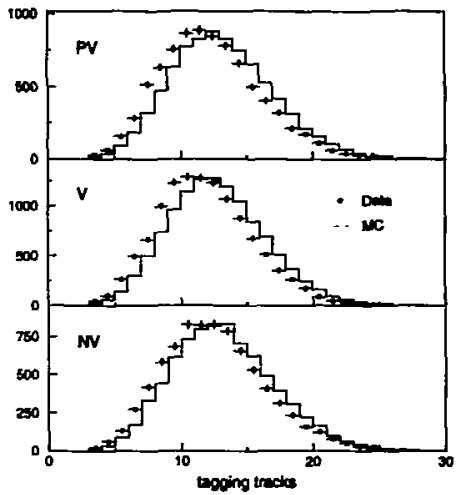

Figure 7.3: The distributions of the number of quality tracks for thase events pacing the badronic selection cuts in the three running periods. The discrepancy betwed the cherged multiplicities between data and MC can easily be seen.

A potentially seriour problem is meked by the normalization of these plots, however, as date and MC bave been normalized wo that the total number of tracks is the same. Figures 7.3n-c display the problem in 2 more telling manner, showing the number of quality tracis $n_{1}$ in the data and MC for those events paring the hedroaic evept selaction cuts. For this generation of MC, the tracking eficiency in simulated events is noticenbly higher than that in the date. This is due to correlated hit low at the edgas of the CDC drift celis, where an entire superiayer's worth of hits can be lost if the track peases too clowe to the field wires. The detrils of the sharge deparition sear the ndge of the CDC cella wese oversimplified in the MC, rewulting in a diference 


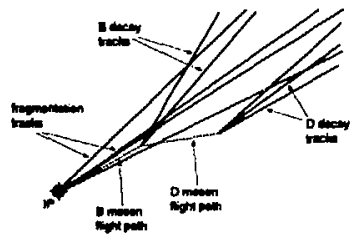

Figure 7.4: Dingram of a $B$ Meson Decay Cascide

bet meen data and $\mathrm{MC}$ in the number of hits avilable for treck finding and fitting. Studies have shown[182] that the track los occurs in an esentially random manner and specifically does not depend on track $p_{1}, \phi_{1} \theta$, or the angle of the track with reopect to the jet axis. Thls is almont certainly due to the randomly uniform location of cell odges within the CDC. So, to infure agreement bettren the MC and data in terms of the number of quality tracks available for tegging, we apply a treck-by-track eficiency correction to all MC events buch that the mean values of $n_{\text {pood agree for }}$ date and MC events. The error on this correction could be arbitrarily small, since the lar be number of tracks in cach sumple results in a tiry error on $\left(n_{\text {pas }}\right)$. To be conservative, however, we compare our MC multiplicity to the world average, sigce wo don't know a priori that the MC has the correct multiplicity. The mean total charged multiplicity at the $Z^{0}$ has been best determined by by ALEPH[183], who measured $20.85 \pm 0.24$ end by OPAL[184], who meseured $21.4 \pm 0.43$. One can see that these two messurements differ by 0.6 tracks, yet are consistent within erron. The average totel charged multiplicity in the SLD MC, 21.1 tracks, is consistent with the these other results, but we will asign a \pm 0.3 tricks etror to the efficiency correction to cover any posable ersor.

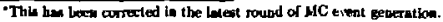




\subsection{The Normalized Impact Parameter}

From this point on, we will refer to the $x y$ distance of clased approach to the IP for a charged treck docan as the iropact perameter d; the mociated error on the quantity is defined $a \sigma_{\mathrm{A}}$. The bacis of the tag method, oripinally suggested by Hayes[185], is that tracks from the decays of mesons containing $c$ and especially b quarts tend not to extrapolate becis to the IP. This is due to two Inctors, the long meson lifetimes and the significast $p_{\perp}$ acquired relative to the meson flighe direction, a result of the large available energy in the heavy quark decay. Figure 7.4 illortrate this point. In principle, a vertex detector mth sulficient resolution should be able to measure the impact parameters of these tracks well enough to alliow a sample of heavy yank events to be selected bued on the number of tracks that mim the IP. As an inproverwent to this techinique, the Mark II collaboration[186] introducod a signed impact paraneter, where now $d$ is positive if the track cranes the meson dighe diraction domnstream of the IP, and negative if it crome the meson tight direction upstreary or behind the IP'. This creates an symmetric distribution, since the trach from heavy quarke tend to populate the region of large positive values of $d$. Fisure 7.5 shows the signing procedure in cartoon form. Since we wimh to be inementive to track mis-keconstruction, the absolute dintence scale, and our imprecine knomledoe of the meson lifetimes, we will use instead the significance of the distance by which each track misces the IP, which we refer to a the pormelbed impact parameter $d / \sigma_{d}$ 프 $d_{\text {narm. }}$. The distributions of $d$ and $d_{\text {more }}$ were nhown in Section 5.3 during the earlier discusgion of trecking performence. Here, we show in Figure 7.6 the dharm distrihution for all tracks from $u d s, c$, and b events in the MC ac an illustration of the different regions that are populated by tracks from events with long-lived heavy mesons.

In this analysis, we have completely decouplat the tagging frow any of the jetfinding ot udies by choosing a fixed definition of the procedure wed to sign the impact parumeters of the tracks. For the event tres, we exploy the JADE jet-finding algorithm[126] with calorineter ciusters as input. The jet rewolution parameter how

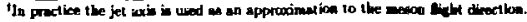




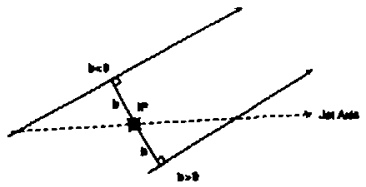

Figure 7.5: Definition of the signed impact parameter. Tracks which crom the jet axis "downatream" of the IP are given positive impact parameters.

of 0.02 is uned to define thene jeth. Several ntudies by the SLD B-phyrics goup[182] heve hown this to prowde the maximum fraction of conrectly-rigned tracks in the emple of hadronic decays.

\subsection{Deflnition of the Flavor Tags}

From the distributions shown in Figure 7.6, one can wee that $b$ and $c$ events do cend to have a lerge fraction of trecke mining the IP by a few sigme. The impact parameter tagging method atlempts to capitalize on thir diffenence to ecparate events with primery light quarks from those with primery beavy quarks. The two variables that define a tag of thin type are the impact paramet:" significence (i.e., how far awy from the IP does the treck heve to be to be have mimed it significantly?) and the number of tracks of that significance in each event (how many track significantly min

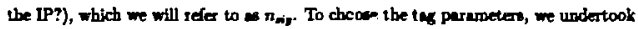
a audy of the eficiencies $\varepsilon^{2}$, puritie $\Pi^{5}$, and tag bieses. Unfortunately, the wearch $\alpha$ \& 27-dimensional epece doesa't bend itself to grephical representation. Since tra of this maner are wentially guaranted to be of bigh eficiency, we seurched for tag

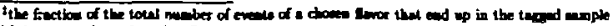

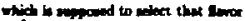

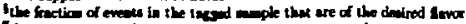

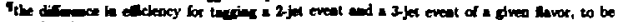
anenow in Cheter
} 


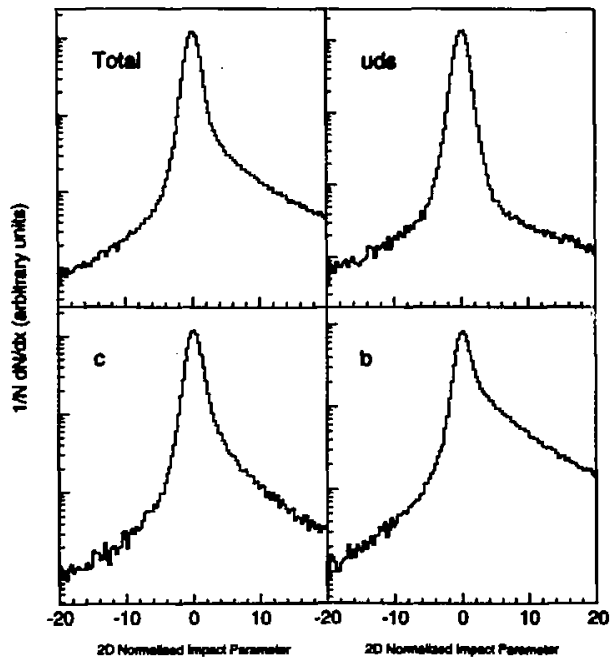

Figure 7.6: The impact parameter diatribution for all tracks in hadroaic eveace, and all tracks in uds, $c_{\text {, }}$ and bevents. The diatributions abown are nomslinad to the totel number of truche in each semple. The long taik at high impect parameters aro readily vinible in the $c$ and $b$ events. 


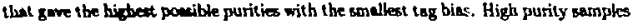
are dedrod becaune large background "subtraction" that occurs when unfolding the propertion of the pure finvor sample from the mix of Bavors in a given tar is reduced, w are the comelatioas bet ween the results for each flavor once the unfolding is compieted. It is interesting to note that, ws the significance cut of the tas is made harder, the b umple becomes more pesre, but the wd purity is diluted. The opposite happens when the significunce cut is lowered unjes a levge $n_{\text {wio }}$ is required. An intermediate cul of a significence of $\mathbf{3 . 0}$ balanoes the nurity in the uds and $b$ samples. Figure 7.7 show the number of tracks with $d_{\text {mon.. }}>3.0$ in each event. The leftmost bin, the one with no niguificant tracks, is almost $90 \%$ uds cvents, while the bins with 4 or more significant tracks are extremely pure in b events. The middle bins contain an euriched cumpte of $c$ events. Onec again, the MC provides a good description of the date for this distribution.

The event sample is divided accordif" ly into thro mutually exclusive tagged subsumples, cach of which will be referred to $m$ the $i^{\text {th }}$ tagged sample:

- those events with $n_{m i g}=0$ were defined to be the udr-tigged sample $(i=1)$

- those with $1 \leq n_{m i} \leq 3$ mere defired to be the c-tagged sample ( $\left.i=2\right)$

- those with $n_{\text {mg }} \geq 4$ were defined to be the b-ticgged sumple $(i=3)$.

For illustration, we list bere a sample set of tas efaciency matrices, for 2- and 3-jet events ${ }^{l}$ which have passed the selection cuts. Tue matrices $c_{n}^{j / j}$ are the efficiencies for tegging an event with $n=2,3$ jets of true flavor $j(j=1: u d s, 2: c, 3: b$ ) with tag i. Hence, the columns coutain the probabilities that an event of a given flavor will be taesed by ewch of the tegs; each column should thus sum to unity.

$$
\varepsilon_{2}^{j J}=\left(\begin{array}{lll}
0.771 & 0.374 & 0.044 \\
0.228 & 0.593 & 0.439 \\
0.001 & 0.033 & 0.517
\end{array}\right) \varepsilon_{3}^{i j}=\left(\begin{array}{lll}
0.732 & 0.411 & 0.072 \\
0.260 & 0.560 & 0.512 \\
0.002 & 0.028 & 0.416
\end{array}\right)
$$

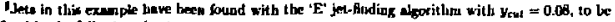
dufied in the following diaptes.
} 
For completeneas, we show also the purity matrice for 2- and 3-jet evept. Here, the rows give the purities of each tas:

$$
\Pi_{2}^{i j}=\left(\begin{array}{lll}
0.865 & 0.117 & 0.018 \\
0.413 & 0.300 & 0.287 \\
0.005 & 0.047 & 0.948
\end{array}\right) \Pi_{3}^{i f}=\left(\begin{array}{lll}
0.839 & 0.131 & 0.030 \\
0.434 & 0.257 & 0.304 \\
0.013 & 0.049 & 0.939
\end{array}\right)
$$

For such a simple tas, wetually do quite well in term of absolute performance: the $b$ tas is about $51 \%$ efficient, with a high purity of about $95 \%$. The excellent reolution of the tracking syotem tumbes this quality of ta easy to achieve.

We mowe on now to a description of the jet-finding thit is performed on these tagged samples to make posible the extraction of the strong coupling. 


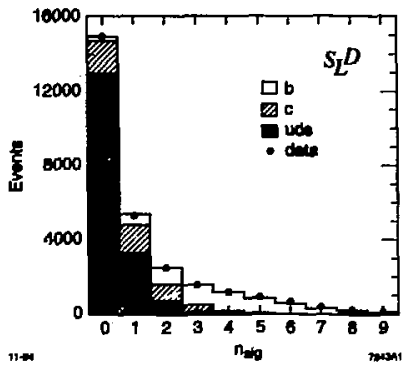

Figure 7.7: The number of tracks $n_{\text {sig }}$ per event which mins the origin by more than $3 \sigma$ in the $x-y$ plane. The solid histogram reprosents the total MC sample, while the points represent the data distribution. The fisvor composition of the dintribution is sivo brivn. (See text.) 


\section{Chapter 8}

\section{Jet Finding}

In order to extract the strong coupling from the final state hadronic structure of $Z^{\circ}$ decays, we must fiset set forth the definition(s) of the jets into which we will oryanize thern.

\subsection{Jet Algorithm Definitions}

As mentioned in Section 3.3.1, jets are defined operationally by ilstative clunterine algorithms. When these algorithms are applied, a meanure m, auch $\boldsymbol{*}$ the inrariant mass, of all pairs of particles $k$ and $l$ in a given exant in calculated, and the pair with the smallest $w_{\text {in }}$ is combined into a single (peetudo)-particle. This proces is repealed until all pairs have vu excesding a value vos. At this point, each peeudo-particle that remains is called a "jet", and the jet multiplicity of the event is just the number of jets.

The differem algorithms are diatinguiabed by their definition of the measure wh and the manner in which the two particles $k$ and $l$ are combined (known $*$ the "recombination scleme"). In the analyzis presented here, we use six of these jet algorithm: the ' $\mathrm{E}$, 'EO', 'P', and 'PO' veriations of the JADE llgorithm]126], * well as the Durham ('D') and Genev ('G') schemes[187]. The definitions of in and the recombination schemes for esch alforithm are presented below"

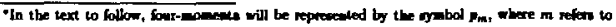




\subsubsection{The E Scheme}

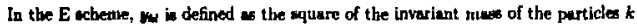
and $l$ nortindied by the rivible erctgy in the event, $E_{\text {wa }}$

$$
y_{u}=\frac{\left(p_{L}+p_{l}\right)^{2}}{E_{v_{b}}^{2}}
$$

The two particles $k$ and $l$ are combined into \& new (poeudo)-purticle $m$ according to

$$
p_{m}=p_{k}+p_{n}
$$

where pion maseax are asumed in culculating the cnergies. Total enera and momentun are explicitly conserved in this mcheme.

\subsubsection{The E0 Scheme}

In the EO wheme, $y_{y}$ is defined by Equation 8.1 , while the recombination wheme is civen by

$$
\begin{aligned}
& E_{m}=E_{k}+E_{t} \\
& \vec{p}_{m}=\frac{E_{m}}{\left|\vec{p}_{k}+\vec{p}\right|}\left(\vec{p}_{k}+\vec{p}\right)
\end{aligned}
$$

Herc, $\bar{p}_{m}$ is rescaled so that pa: icle $n$ has zero invariant mass. This scherne does not conserve the total momentum in in event.

\subsubsection{The P Scheme}

In the $\mathrm{P}$ mcheme, $\mathrm{y}_{\mathrm{k}}$ is given by Equation 8.1 and the recombination is defined by

$$
\begin{aligned}
& \vec{p}_{m}=\vec{p}_{k}+\vec{p} t \\
& E_{m}=\left|\vec{p}_{m}\right| .
\end{aligned}
$$

This entheme does not conserve the total energy of an cvent, but does conserve the total momenturn surn.

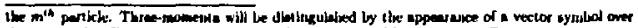

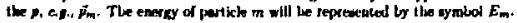




\subsubsection{The P0 Scbenve}

The P0 scheme identical to the P wcheme, except that the viwible cners $E_{\text {nd }}$ in Equation 8.1 is recaleulated after each iseration moonding to

$$
E_{w+}=\sum_{m} E_{m} .
$$

\subsubsection{The D Scheme}

In the D acheme, the parameter $/ / w$ is defined $*$

$$
\nu_{M}=\frac{2 \min \left(E_{h_{1}}^{2}, E_{?}^{2}\right)\left(1-\cos \theta_{m}\right)}{E_{m}^{2}},
$$

where $f_{k}$ in the angle betwen partkles $k$ and $l$. The recombination wheme is given by Equation 8.2. From an inspection of Equation 8.6, one can see that the D aloorthen will tend to cluster wof perticles with other nearby wh particles, a property sot whed with the JADE-derived algorithen, which ofien anociete coft perticlas with a jet in the oppoeite thrat beminplere.

\subsubsection{The G Scheme}

The definition of $y_{w}$ for the $G$ scheme is

$$
y_{d t}=\frac{8 E_{1} E_{l}\left(1-\cos \theta_{w}\right)}{\mathscr{g}\left(E_{k}+E_{t}\right)^{2}} .
$$

The recombination scheme is aloo given in thin ine by Equation 8.2. Note that the G acberne has the same properties vis-a-vis sof particles as the D schene, but in this cas $y_{k}$ onj; depends on the enerpits of the two particles under consideration, not on the total $E_{\text {tio. }}$

\subsection{Definition of the $n$-Jet Rate and $D_{2}$}

In determining $a_{*}$ from QCD predictione, it is of course the rate of multi-jet events that matten, not the number of jets in each event. So, define tho n-jot rate 


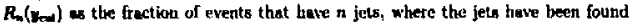
wing a jet algorithm with inges cut-off tow. For purposes of illust tration, we slow in Fizure $8.1 R_{n}\left(y_{c r}\right)$ Ior $n=2,3,4,5$ for all of the algorithtns, wivere the jnts linve been found using clurged tracks in the detector.

Since ecli of the points at each value of you in Figure 8.1 is deternined using the entire date wet, all the points show are highly correlater. One can remove this correlation by defining the differential 2-jet rate, $D_{2}[188]$

$$
D_{2}\left(y_{\text {ax }}\right)=\frac{R_{2}(m)-R_{2}\left(y_{\text {at }}-\Delta y_{\text {aut }}\right)}{\Delta y_{\text {mat }}} .
$$

An event will only contribute to the $D_{2}$ distribution if it is redefined as a 2-jet event instead of a 3-jet crent as the salue of $y_{\text {out }}$ is clanger from yout to $y_{\text {rot }}-\Delta y_{\text {act. }}$. Sample $D_{2}$ diatributions will be shown in Section 9.3 und at the end of the present chapter.

\subsection{QCD calculations for Jet Rates and $D_{2}$}

As toentioned in the introduction, purturbative QCD calculations which relate observable quantitics to the strong coupling a, hese been perforsued for all of the jet-finding algorithrns and the $D_{2}$ distribution defined above. In the hext two sections, we discuss these calculations and their implications for the prospects of determining $a_{a}$.

The geters! form of the jredictions for the 3 -jet rat: $H_{3}[128,129]$ was presented in Section 3.3.1 and is reproduced here lor relerence:

$$
R_{3}\left(y_{\text {at }}\right)=\frac{\alpha_{d}(\mu)}{2 \pi} A\left(y_{\text {out }}\right)+\left(\frac{\alpha_{s}(\mu)}{2 \pi}\right)^{2}\left[B\left(y_{\text {out }}\right)+A\left(y_{\text {rut }}\right) 2 \pi b_{0} \log f\right] .
$$

Hore $\mu$ is the renormalization scale defined in Section 3.1.3, and $f=\mu^{2} / 8$. Here. $b_{0}=\left(33-2 n_{j}\right) /(12 \pi)$, where $n_{f}$ is dise tumber of active flavors $\left(n_{f}=5\right.$ at $\left.\sqrt{s}=M_{z}\right)$. The terms prowortional to $a_{8}^{2}$ include contributions $(B)$ from four-jet uvents where two of the ets were sufficiently close to be unresolvable at the chosen value of $y_{\text {out }}$. The culculaion rolating $D_{2}$ to $a$, is somewhat unssis, as it requires differentiating the expression in Equmlion 8.9 with respect to itint cnd thus has no analytic cloeed form. The 4-iot rate $d_{1}$ lusi the form has only been culculut od to loweat order[87], asd has 


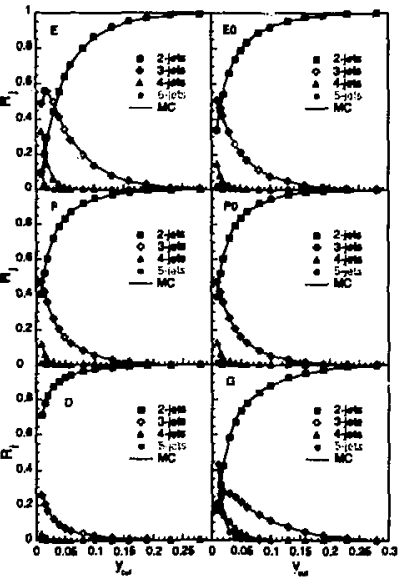

Figure B.1: Plots showing the fructional jet rates for euch of the 6 jet ajgnithms. The various points represent the $n$-jet rates, and the solid curves are the sa. quantities derived fron the MC simulation of hadronic $Z^{0}$ decays. 
the expected form:

$$
R_{1}\left(y_{\text {cut }}\right)=\left(\frac{\alpha_{s}(\mu)}{2 \pi}\right)^{2} C\left(y_{\text {cat }}\right) .
$$

In general, the coefficicnts $A\left(y_{\text {cad }}\right)$ und $C\left(y_{\text {oul }}\right)$ are the same for all algorithms, since there should be no dependence of the lowest ordor result on the choice of some specific jet-finding algorithm. The three eveffieients $A, B$, and $C$ were evaluated using the EVENT progran developet by Kunszt and Nason[128], snd tables of the value obtained are presented in Appendix $B$. Typical viues of $A, B$, and $C$, for example for the $E$ algorithm at $y_{\mathrm{cul}}$ of 0.08 , nre Note ugain that the renormalization scale $\mu$ appears in the formula for $\boldsymbol{R}_{3}$. Its clfact on the analysis will be diseussed in Chapters 9 and 11.

We find in examining the results of the calculations that the 4-jet rate predicted by Equation 8.10 is not that observed in the dat a for all but the $\mathrm{G}$ algorithm. We need to increase the coefficient $C\left(y_{\mathrm{out}}\right)$ by $\sim$ a factor of two for all of the other jet algorithms to obtain good agreement with the data. The full range of this variation will be taken as the systematic crror due to the uncertainties in this calculation.

\subsection{The Need for Corrections}

It should be stressed again that the perturbative QCD calculations described in the previous section refer to quantities caleulated ut parton-level in the theory. Ideally, the measurement of some global property of hadronic tvents using the final state hadrons should not be effected by the hadronization process itself. Unfortunately, losses due to such things as detector acceptance, non-perfect tracking and calorimetry, and the need to select fiducial events from a sample contuning hackground all imply that the global properties measured with the actual detector apprsatus will not be the same as those exhibited by the underlying partons. The typical remedy[131] to this situation is to apply corrections, either bin-by-bin over the distribution in question or using the full bin-to-bin correlation matrix, to "correct" the measured data distribution for all of its mistrentment, resulting in a sample thrt should resemble the true parton distribution. This requires, of course, a believable dutector $M C$, as well as some model 
of the hadronization process. Examples of correctel $D_{2}$ distributions, which can tben be compared directly with the theoretical predictions, are shown in Section 9.3. In this snalysir, we will also need to make correctiuns of this kind, and thus require good agreenent between MIC and data for the distributions of interest. In order to demonstrate the quality of the MC deacription of the detector, $w$ show in Figures 8.2-8.4 the $D_{2}$ distributions for the tagged samples in the three run periods. The MC and data agree quite well over the many decades of these distributions. We will not actually we the $D_{2}$ distribution to extract $\alpha_{\text {f }}$ for the different quark favors, but it serves here as a good cross check and gives us confidence that we underatand the effects of the detector on the jet rates well enough to attempt the neoded corrections. The methods used for the corrections will be deacribed in the next chapter. 


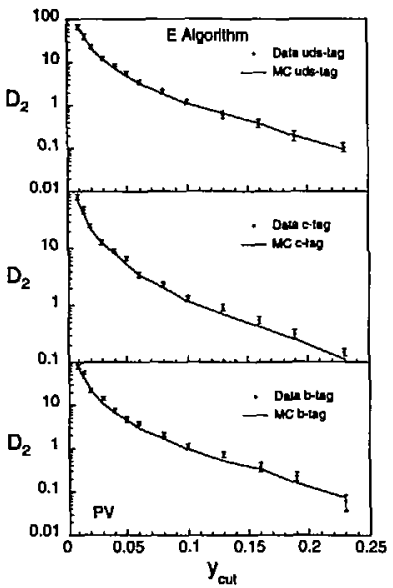

Figure 8.2: The $D_{2}$ distribution for the data (points) and MC (line) uds, $c_{1}$ and b-tagged samples. The duta and MIC are both tuken from the Pre-velo run period. 


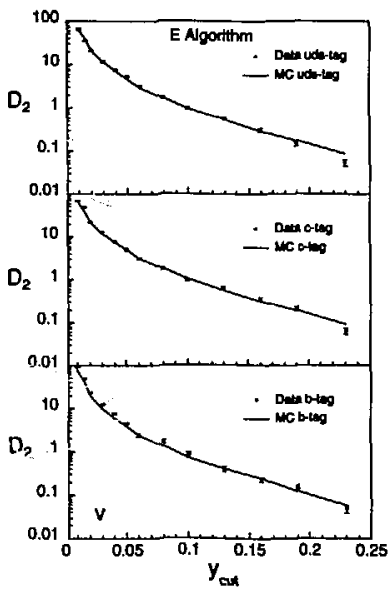

Figure 8.3: The $D_{2}$ distribution for the data (points) and MC (line) uds, $c$, and 6-tugged susnples. The data and MC are both taken from the Veto run period. 


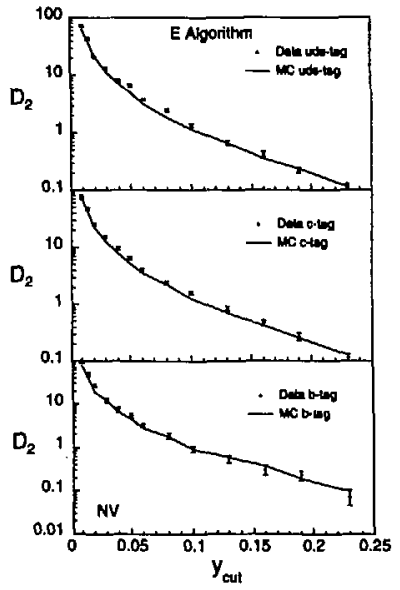

Figure 8.4: The $D_{1}$ distribution for the data (points) and MC (line) uds, $c$, and b-tsgged samples. The dain and MC are both taken from the Non-veto run period. 


\section{Chapter 9}

\section{Analysis Method}

Now that the tools for flavor tagging and jet-finding have been prosented, we can move on to a discuesion of the method by which we can extract $a$, for each quark flevor. This chapter presents a detailed discussion of the choice of method and the mathematical formulation of the unfolding and parton-level-correction procedure, and ends with the results for the jet rates deternined for each quark flavor.

\subsection{Considerations}

From the Inception of this project, it has been clear that the most precise toot of the flavor-independence of strong interactions must come from messuring a ratio of the strong couplings of one quark flavor to another or to the entire sample. The ratio must be formed in a way such that the uncertainties due to detector effecta, hadronization models, and renornalization scale uncertainty brgely cancel. If thin in not the case, then the test of flavor-independence will be plagued by the same large sources of theoretical uncertainty that effect an abolute determination of $\alpha_{n}$. These problems were discussed in some detail in Chapter 3 . Until this work, the atendard practice" has been to isolate an enriched sample of the quark flavor of interest and then to apply some combination of the techniques discusaed in Chapter 3 to manure $\alpha$, for that particular quark flavor, which ts then compared to $\alpha$, measured from

Fee Section 3.4.I 
the whole sample of hadronic decays using the same tixthiques. This requites one to make the parton-to-hadron and hudron-to-detectur corrections separately for the lagged ample and the whole set of hadronic derays, and then hope that they (and hence there uncertainties) cancel to a large oxtent when tho ratio is formed. This muy not be the case for events contaittius hervy yluurks, for example, which then linve to be given special treatnent. In addition, some waunption must be mode about the value of $a_{a}$ for the background in the tag sample; since no tag produces samples of 100\% purity. One can attemph, as huve the OPAL collaboration[195], to loosen thesen assumptions by combining the results from an exchusive, low efficiency tag for each Havor and unfold the ratios of couplings for puru cpark samples. This works, but lang some large errors from the unfolding yroccklure tssociated with it.

Instead of pursuing this minthod, we have chosen to pursue an inclusive tagging analysis, where we use all hadronic ovents in our fiducial sample. We have secn in Chapter 7 that the multiplicity of signiticant-impact-parnmeter tracks provides a clean tag of uds and $b$ evcuts nud some separation for $c$ events when the entire event sample is broken into the three tagged samples defined there. This tag definition is Lнaed on ял "inclusive" varial)le like the number of tracks, rather than something "exclusive", like requiring fast protons. Hencu, one can be morc certain that the eftciency matrices used to unfold the tagged distributions into results for pure flavor samples are insensitive to the Inimute detuils of the MC.

\subsection{The Choice of $R_{3}$}

\section{as the Analysis Variable}

Once the flukor seputution hes been yerforned, one is left with the task of act ually

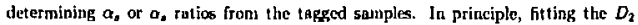
distribution would be the obvions choice, since it maximizes the statistical wejght of ench event. However, to avoid uncertainties due to the large corrections necessary to obtain the parton level distributions for each tagged sample and the globn! event yample sepnrately, we renlly would like to fit the ratio of the corrected values as a 
function of $y_{\text {sut }}$. This proves to be a somewhat tangled undertaking, as both diotribution shape and normalization information bscome relevant to the fit. In addition, we would then be fitting the ratio of two second derivatives, which docan't exactly lead to a clear, intuitive graply of the problens. If we add to this the difficulty that the fitting apparatus becones extrumely complicated and hard to understand in a etraightforward manner, we are driven to search for a more elegant means of extracting $a$, from the data.

This is provided by returning to the 3 -jet rate $R_{3}$ as the basis for the analyois. Calculation of $R_{3}$ for ench of the tapged somples is a simple taok, and if jets are found at a single value of $y_{c u t}$ for each algorithm, there is no double counting of ovents, We have in Equation 8.9 н perturbative QCD calculation for $R_{3}$ relating the parton-level jet rate to $\alpha_{3}$, so we can actually perform a messurement with this observable, and a ratio of the threejct rate for a tagget sample to that of the entire event sanple can easily be formed. Thls also provides las with a more intujtive variable space in which to perform the analysis. In the these of jet rates, calculations of the effects of heavy quark musses on the phese space for hard gluon emission luve been performed, and we cun use them to correct our observed distributions for the kinematic differences of heavy quark events. As we shall sec, this choice of method allows us to proceed in series of well-defined steps from the tagged distribution to the final ratios of $a_{\text {s }}$ values.

\subsection{Choosing the Values of $y_{c u t}$}

In order to perform the jet-rates analysis, we need to define what we mean by a "jet" for each jet-finding alyorithms discussed in Chapter 8. This involves the choice of the jet resolution paraneter $y_{\text {ous }}$, which gives the maximum allowed invariant maks of a pair of (pseudo)-particles such that they will be combined into a aingle cluster. Originally, we worrits about the uncalculated ligher orders in the QCD predictions for $R_{3}\left(y_{\text {cut }}\right)$, thinking they would introduce large theoretical uncertalntios. To wueliornte this problem, we chose at that tine ycuavelues such that the 4-jet rate $R_{4}$ (yoi)

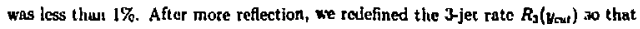


$R_{3}\left(y_{a x}\right) \equiv R_{3}\left(y_{c o t}\right)+R_{s}\left(y_{c u t}\right)+R_{s}\left(y_{\text {cut }}\right)+\ldots{ }^{\prime}$, as it turned out that the additional uncertainty in doing this is small ${ }^{4}$. Now, we are free to try and minimizo the statistical error on $\boldsymbol{R}_{3}$, by choosing a you vulue that givis the largest number of 3-jet events. We must be wary, though, of sclecting yeut in a region of parametor specco where the data are not well described by the $O\left(a_{2}^{2}\right)$ calculations thet we wish to use to extract $\alpha_{n}$, or where the decay products of henvy quarks are split inte different jols. A solution to the problem is provided by the parton-level-corrected $D_{2}$ distributions calculated by Ohnishi[131]. Figures 9.1 through 9.6 show the partun-correctod data plotted on top of the full $\mathcal{O}\left(a_{3}^{2}\right)$ calculution. The deviations of the data and the theory at low yoraccur when the contributions to the 2-jet rate from the unsisalon of many soft gluons becomes important. One can see that this happens at different values of $y_{\text {act }}$ for each algorithm, as can be expectod. Since it is this $O\left(a_{0}^{2}\right)$ calculation that we would like to use to extract $\alpha$, foi this analyxis, we are free to choose the lowest value of yout where the data is well-described by the theory. The chosen $y_{\text {cout }}$ values are denoted by the labe! "this analysis". Thesc valuer also appear in Table 9.3. Thest figures also display the hadronizution $\left(C_{H}\right)$ and detector acceptance and resolution corrections $\left(C_{D}\right)$ that were appliut to the date to obtain the parton-level distributions. The fit ranges that were used for extracting $a$, from fitting the $D_{2}$ distributions nre also shuwn. For some algoritlums, these are smaller than the full sunge over which the theory appears to agtoe with the data due primarily to the size and uncertainty in the applied corrections $C_{H}$ and $C_{D}$. We are free to explore a larger range of $y_{\text {cut }}$ as wo expect the uncert binty due to tlice corrections to be relntively small in this analysis.

\subsection{Fitting the Data for $R_{3}$}

Here, we present the mathematical formulation of the fit which allows us to extract $\boldsymbol{R}_{3}$ at the parton-level for eacls quark favor from $\boldsymbol{B}_{3}$ in each of the tagged samples. The parton-level 3-jet rate $R_{3}^{j}$ for each of the $j$ quark types $(j=1: u d s, j=2: c$,

\footnotetext{
Nole that thlo buyplies that $\Omega_{2}=\bar{l}-R_{2}$.

this will be discused in mone detall in Chnpters 10 and 11 , but tha eflect is $\sim 0.3 \%$.
} 


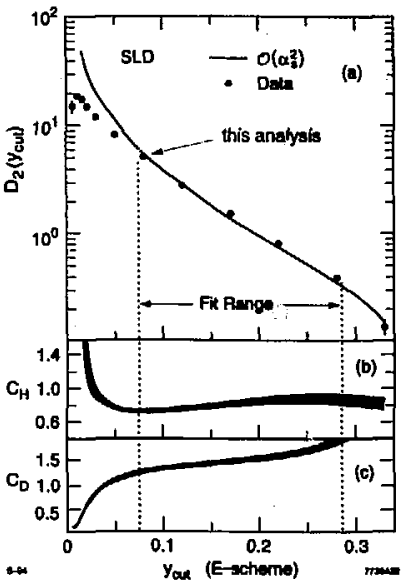

Figure 9.1: The $D_{2}$ Distribution and $O\left(\alpha_{*}^{2}\right)$ Calculation for the $E$ algorithen. The grey bands show the range of uncertainty on the corrections which would be applied to the data to obtain the proper "hadror"-lavel $\left(C_{b}\right)$ and parton-level $\left(C_{H}\right) D_{2}$ distributions if one wanted to extrect a value of $\alpha$, from this distribution. The solld curve in the top plot is the $O\left(a_{1}^{2}\right)$ QCD calculation of $D_{2}$. The artow show the valuo of yew selected for this analysbs (sec text). 


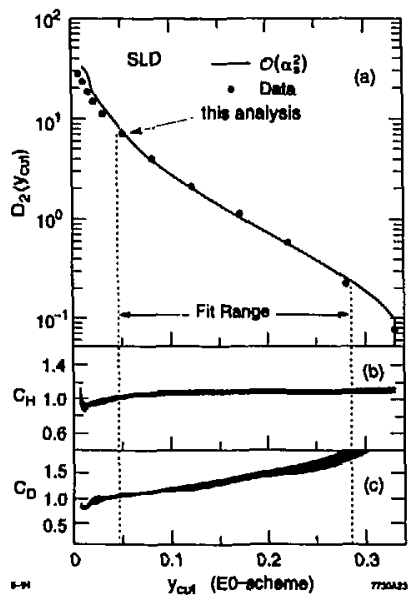

Figure 9.2: The $D_{1}$ Distribution and $\mathcal{O}\left(\alpha_{3}^{2}\right)$ Calculation for the E0 algorithm. See the caption of Figure 0.1 for details. 


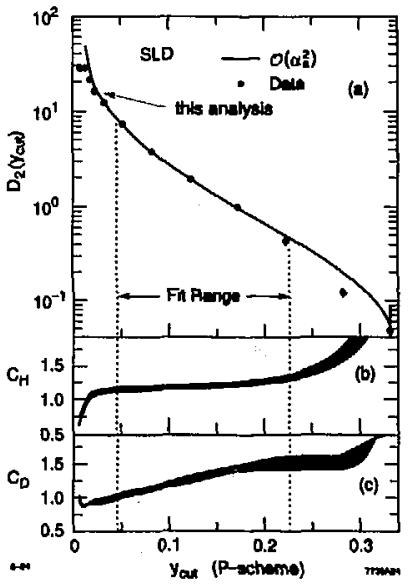

Figure 9.3: The $D_{2}$ Distribution and $\mathcal{O}\left(\alpha_{n}^{2}\right)$ Calculation for the $\mathrm{P}$ algorithn. See the caption of Figure 9.1 for detrils. 


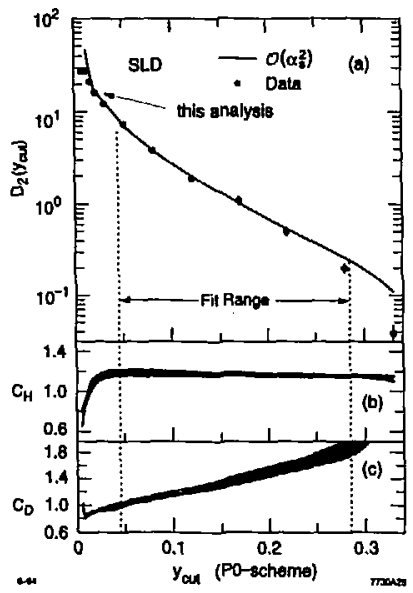

Figure 9.4: The $D_{2}$ Distribution and $O(\alpha)$ Calculation for the P0 algorithm. Sec the caption of Figure 9.1 for details. 


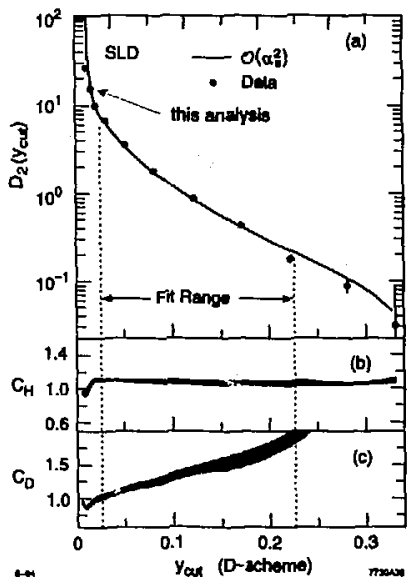

Figure 9.5: The $D_{2}$ Distribution and $O\left(\alpha_{f}^{2}\right)$ Calculation for the $\mathrm{D}$ algorithm. See the caption of Figure 9.1 for details. 


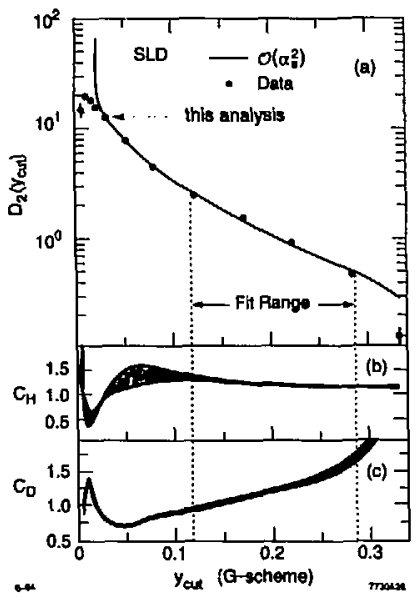

Figure 9.6: The $D_{2}$ Distribution and $O\left(a_{\text {? }}^{2}\right)$ Calculation for the $G$ algorithm. See the caption of Figure $\mathbf{9 . 1}$ for details. 
and $j=3: 6$ ) end for each jet slgorithin wns extracted from a maximum likelihood fit to the following expresions for $n_{2}$ and $n_{2}$, the number of 2 -jet and 3 -jet evests, respectively, in the $i^{\text {th }}$ touged sample:

$$
\begin{aligned}
& u_{2}^{i}=\sum_{j=1}^{3}\left(\varepsilon_{(3 \rightarrow 2)}^{j j}\left(1-R_{3}^{j}\right)+\varepsilon_{(3-2)}^{j j} R_{3}^{j}\right) f^{j} N \\
& n_{3}^{j}=\sum_{j=1}^{3}\left(\epsilon_{(3 \rightarrow 3)}^{j j} R_{3}^{j}+\varepsilon_{(2 \rightarrow 3)}^{j j}\left(1-R_{3}^{j}\right)\right) f^{j} N .
\end{aligned}
$$

Here $N$ is the total number of selected events corrected for the efficiency for a hudronic $Z^{0}$ decay to pass all event selection cuts, which effectively makes $N$ the total number of parton-level events that would correspond to observing $n_{2}^{i}+n_{j}^{i}$ data events after cuta and detector scceptance. The symbol $f J$ is the Standard Model fractional hadronic width for $Z^{0}$ decays to the $j^{\text {ih }}$ quark type, i.e.,

$$
\rho^{2}=\frac{B R\left(Z^{0} \rightarrow q, \bar{q}_{j}\right)}{B R\left(Z^{0} \rightarrow \text { hadrons }\right)} .
$$

The matrices $\varepsilon_{(2 \rightarrow 2)}^{i j}$ and $\varepsilon_{(3 \rightarrow 3)}^{(j)}$ are the efliciencier for an uvent of type $j$ containing 2- or 3-jets at the parton level to pass all cuts and be tagget an a 2- or 3-jet event, respectively, of type $i$. The matrices $\varepsilon_{(2 \rightarrow 3)}^{i j}$ and $\varepsilon_{(1-2)}^{i j}$ are the clficiencies for an event of type $j$ containing 2- or 3 -juts at the parton level to pass all cuts and be tagged as a 3- or 2-jet event, respectively, of type i. We remind the rcader that, by definition, $\boldsymbol{R}_{\mathbf{2}}=1-\boldsymbol{R}_{\mathbf{3}}$. The efficiency matrices e were calculated from the MC by performing the jet-finding at the parton level, then subjecting the events to the flavor tag and jet-finding at the detector level. One can think of the matrices $\varepsilon$ for 2-and 3-jet events as being the multiplicative product of three efficiency nuatrices

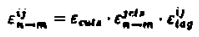

where the first two matrices are diagonal, sinec only the flovor lagging mixes the different quark species. The first matrix, ente, contains the efficiencics for the events of the different quark flavors to pass the trigger and event selection cuts. The second matrix keps track of the migration of the parton-level jets into the obecrvable final states in the deteclor, and the third matrix is a measure of the efficiency for tagging 
the eppropriatc final state. In general: all threc of those matrices are different for 2- and 3-jet events. Values for $\varepsilon_{\text {now }}$, were given at the end of Chapter 6 , and sample tagging efficiency matrices $\varepsilon_{i j}^{\text {lag }}$ were given in Ghapter 7 . For completeness, the four efficiency matrices for each jet algorithm and the mathematies for performing the fit are contained in Appendix A. The determination of these matrices are the heart of this anadysis; virtually all bystematic encors cun be expresced as a variation in asme or all of their clements.

One can see that this formalism explicitly accounts fur modifications of the partonluvel 3-jet rate due to hadronization, detector effects, and tagging bias. Since the jet retes for all three flavore are fit sirnultancously, there are a'bo no assumptions made ubout the vajues of the strong couplings for cach quark type. In fact, the total 3-jet rate $R_{3}^{\prime \prime}$ is just given by

$$
R_{3}^{\text {alt }}=f_{\text {rate }} \cdot R_{3}^{\mathrm{dat}}+f_{c} \cdot R_{3}^{\mathrm{c}}+f_{b} \cdot R_{3}^{b},
$$

$\infty \mathrm{it}$, too, comes from the fit.

Note that, by choosing to formulate the problem in terms of efficiency matrices, we do not depend on the MC to properly reproduce the rate of 3 -jet production and thus this analysis is only weakly dependent on the choice of $\alpha$, used in generating the MC Bample.

\subsection{Tag Bias}

We pause bricfly in the description of the analysis to discuss the bias of these tegs towards selecting 2-jet events preferentially cver 3-jet nvents. This can happen for a nutmiser of reasons depending on the tag in question. For example, requiring a high-p, high-p $p_{1}$ lepton to lag $b$ events tends to tag those events where the $b$ has acquired a large fraction of the available energy from the $Z^{0}$ decay. Since the $b$ hadrons in evonts with hard gluon radiation are less energetic, they are tagged less frequently by thiy trg, and the tag is therefore biased toward selecting 2-jet events. To quantify this, we dufine the tag bias $B^{\prime}$ as the difference from unity of the ratio of the $i^{\text {th }}$ diagonal 
elements of the 2- guld 3-jut efficiency matrices:

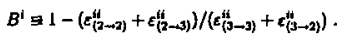

Averaged over the six jet algorithms, the biases are:

$$
B^{\text {wh }}=0.057 \pm 0.005, B^{C}=0.083 \pm 0.016, B^{4}=0.303 \pm 0.037 \text {, }
$$

where the errors repreacrt the r.m.s variations. In contragt to the previoualy employed tagsi, the tag biases for uds and $c$ events are relatively small. In general, lifetime tags of the type we use here are sensitive to the average impact parameter resolution of the ensemble of tracks in the desired event. This provides the koy to understanding the tag biases mentioned sbove. As the gluon in a 3-jet b event becomes more and more energetic, the recoiling $b$ hadrons carry less and less energy. Since the impact parameter resolution rapiclly becomes large for lower momentum purticles, the tracks from the decay of these softer bo will be less likely to have a sigulficant impact parameter because the measurenent of their trajectories will be leas precise. The smaler boost of the $b$ and the cotresponding docrease in average decay length also cantribute to the inefficiency for tagging 3-jet cvents. The differences in the case of uds events can be mainly attributed to event containment effects, as there is bome lows of efficiency when one of the jets exits the detector along the beam pipe.

As mentioned above, these biave are explicitly corrected by the matrix unfolding procedure. It is intereating to note the variation in bias emong the differcnt algorithros and among the Pre-Veto, Veto, and Non-Veto run periods. Table 9.1 tists all of these values. One can see that the inherent bias in this method, given by the Pre-Veto and Non-Veto sarnples, is much sunuller than those quoted in Equation 9.6, even for the $b$ tag. The track trigger veto hus drastically increased the bias of each of the tags by preferentially vetoing 3 -jet events. Since we believe that the effects of the veto are properly modeled in the MC, the corrections we apply in the unfolding fit should still yield the correct parton-level distributions.

\footnotetext{
Sgec Section 3.4.1.
} 
Table 9.1: The tag binses, as defined in Eq. 9.5 for each algorithm and esch running period.

\begin{tabular}{|c|c|c|c|}
\hline Jet Algorithm & $B^{\text {udd }}$ & $B^{c}$ & $B^{b}$ \\
\hline Pro-Veto: & & & \\
E & 1.012 & 1.041 & 1.244 \\
E0 & 1.019 & 1.017 & 1.203 \\
P & 1.024 & 1.041 & 1.172 \\
P0 & 1.027 & 1.039 & 1.172 \\
D & 1.028 & 1.049 & 1.266 \\
G & 1.039 & 1.057 & 1.214 \\
\hline Veto: & & & \\
E & 1.175 & 1.259 & 1.621 \\
E0 & 1.159 & 1.204 & 1.511 \\
P & 1.139 & 1.202 & 1.474 \\
P0 & 1.143 & 1.215 & 1.477 \\
D & 1.182 & 1.257 & 1.670 \\
G & 1.135 & 1.213 & 1.456 \\
\hline Non-Veto: & & & \\
E & 0.969 & 0.975 & 1.151 \\
E0 & 0.967 & 0.961 & 1.138 \\
P & 0.979 & 0.949 & 1.137 \\
P0 & 0.975 & 0.951 & 1.137 \\
D & 0.972 & 0.957 & 1.170 \\
C & 0.978 & 0.952 & 1.124 \\
\hline
\end{tabular}




\subsection{Results for $R_{3}^{j}$}

Table 9.2 lists the number of events for each run period that make up the inputs to the unfolding procedure defined in Equation 9.1. The MC sample used for this analyois consists of 286,764 events, divided into 102,360 Pre.Veto evento, 137,773 Veto events, and 46,631 Non-Veto everts. We solve Equation 9.1 for each of the six jet algorithrs for the Pre-Veto, Veto, and Non-Veto periodo separately', The results are then combined by taking the proper weighted average. The extracted 3-jet rate ratios $R_{3}^{j} / R_{3}^{a y}$ are shown in Table 9.3, where the stetistical errors on each $R_{3}$ value have been chtained using the full covariance matrix. Note that, since the seme events are used to obtain each of these result, the value are highly correlated. Since we are unfolding these values from a single $n_{\text {dig }}$ distribution containing all of the events, correlations are induced between the values obtained lor each of the quark flavors. Averayed over all six jet algorithins, the correlation coefficients from the fit are: udsc: $-0.76 \pm 0.02$, uds $-b: 0.30 \pm 0.02, c-b:-0.55 \pm 0.01$, where the errors are the r.m.s. variations. They follow the pattern one might expect: since the uds and $b$ samples are relatively pure, the correlation between the malus obtained for these two samples should be small. The correlation between the $c$ result and those for the other samples is large due to the much lower purity of the charen ample, which means that the result for the 3-jet rate for $c$ quarks is extremely gensitive to the trils of the $b$ and $u d s n_{\mu g}$ distributions in the $c$ tag region.

The next chapter will bring us from these raw cxtracted jet rute rutios to the final result.

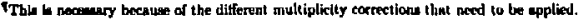


Table 9.2: The number of 2- and 3-jet events tagged by each tag for ench algorithm and run period. These are the $n_{1}^{j}$ and $n_{3}^{j}$ that are input to the fit in Eq. 9.1.

\begin{tabular}{|c|c|c|c|c|c|c|}
\hline Jet Algorithm & $n_{2}^{W^{\alpha}}$ & $n_{2}^{c}$ & $n_{2}^{\frac{1}{2}}$ & $n_{3}^{2+2}$ & $\pi_{3}^{\mathbf{c}}$ & $n_{5}^{b}$ \\
\hline $\begin{array}{c}\text { Pre-Veto: } \\
\text { E } \\
\text { E0 } \\
\text { P } \\
\text { P0 } \\
\text { D } \\
\text { G }\end{array}$ & $\begin{array}{l}3485 \\
3447 \\
3157 \\
3063 \\
3428 \\
2493\end{array}$ & $\begin{array}{l}2082 \\
2102 \\
1919 \\
1856 \\
2074 \\
1494\end{array}$ & $\begin{array}{l}804 \\
815 \\
746 \\
711 \\
806 \\
636\end{array}$ & $\begin{array}{c}963 \\
1001 \\
1291 \\
1385 \\
1020 \\
1955\end{array}$ & $\begin{array}{c}734 \\
714 \\
897 \\
960 \\
742 \\
1322\end{array}$ & $\begin{array}{l}220 \\
209 \\
278 \\
313 \\
218 \\
368\end{array}$ \\
\hline $\begin{array}{l}\text { Veto: } \\
\text { E } \\
\text { EO } \\
\text { P } \\
\text { P0 } \\
\text { D } \\
\text { G }\end{array}$ & $\begin{array}{l}5366 \\
5320 \\
4918 \\
4810 \\
5297 \\
3999\end{array}$ & $\begin{array}{l}2981 \\
2971 \\
2700 \\
2985 \\
2960 \\
2274\end{array}$ & $\begin{array}{c}1084 \\
1086 \\
1025 \\
994 \\
1098 \\
874\end{array}$ & $\begin{array}{l}1169 \\
1215 \\
1617 \\
1725 \\
1238 \\
2536\end{array}$ & $\begin{array}{c}722 \\
732 \\
943 \\
1018 \\
743 \\
1429\end{array}$ & $\begin{array}{l}209 \\
207 \\
268 \\
209 \\
195 \\
418\end{array}$ \\
\hline $\begin{array}{c}\text { Non-Veto: } \\
\text { E } \\
\text { E0 } \\
\text { P } \\
\text { P0 } \\
\text { D } \\
\text { G }\end{array}$ & $\begin{array}{l}3237 \\
3217 \\
2940 \\
2823 \\
3177 \\
2276\end{array}$ & $\begin{array}{l}2014 \\
2009 \\
1836 \\
1778 \\
1980 \\
1455\end{array}$ & $\begin{array}{l}794 \\
800 \\
733 \\
718 \\
789 \\
637\end{array}$ & $\begin{array}{l}1042 \\
1062 \\
1339 \\
1456 \\
1102 \\
2003\end{array}$ & $\begin{array}{l}714 \\
719 \\
892 \\
950 \\
748 \\
1273\end{array}$ & $\begin{array}{l}182 \\
176 \\
243 \\
258 \\
187 \\
339\end{array}$ \\
\hline
\end{tabular}


Table 9.3: The results for the ration $\boldsymbol{A}_{\mathbf{3}} / \boldsymbol{F}_{5}$ obtained in inverting Equation 9.1 for each of the jet-finding algorithms uned witb the given $y_{\text {ou }}$ walue.

\begin{tabular}{|c|c|c|c|c|}
\hline Algorithm & $\psi_{\epsilon}$ & $R_{3}^{2} / R_{3}^{\prime \prime}$ & $\boldsymbol{R}_{3} / \boldsymbol{R}_{3}^{w_{3}^{\prime \prime}}$ & $R_{3}^{b} / R_{3}^{N / N}$ \\
\hline & 0.080 & $0.941 \pm 0.042$ & $1.212 \pm 0.173$ & $0.990 \pm$ \\
\hline E0 & 0.050 & & .145 & $0.981 \pm 0.053$ \\
\hline & 0.030 & $1.001=$ & 0.109 & $1.007 \pm 0.041$ \\
\hline P0 & 0.030 & $1.014 \pm 0.026$ & \pm 0.102 & $1.037 \pm 0.039$ \\
\hline D & 0.015 & 0.989 & $1.096 \pm 0.145$ & $0.947 \pm 0.049$ \\
\hline G & 0.030 & $1.032 \pm 0.020$ & $0.942 \pm 0.079$ & $0.852 \pm 0.030$ \\
\hline
\end{tabular}




\section{Chapter 10}

\section{From Jet Rates to $\alpha_{s}$}

This chapter brings to a close the description of the methods used in this andyeis. Herc, we will discuss the corrections that must be applied to the measured 3-jet rates to account for the sup?" "cssion of hard gluon radiation due to the heavy querk mases. Then, we will present 'se firnl gtep in the extraction of the $\alpha$,velues for each of the quark flavors, the conversion of the ratios $H_{j}^{J} / R_{3}^{2 l}$ into the ration $\alpha_{s}^{j} / \alpha_{*}^{\prime \prime}$.

\subsection{Phase-Space Suppression of Gluon Radiation in Heavy Quark Events}

The 3-jet rate in heavy quark $(b, c)$ events is expected to be reduced relative to that in light quark events by the diminished phase-space for gluon etriosion duc to the heavy quark masses. This was first pointed out by loffe[189/, and further explored most resently by Ballestrero, Maina, and Moretti|191]. This is exactly analogous to the Inass effects on [192] the rates for the processes $e^{+} e^{-} \rightarrow \mu^{+} \mu^{-} \gamma$ and $e^{+} e^{-} \rightarrow \tau^{+} \tau^{-} \gamma$ compared to that for $c^{+} c^{-} \rightarrow e^{+} e^{-} \gamma$ in QED. In the QED calculation, the croses section for the radiative decaye is a function of the "observabitity" of the ploton in a detector, i.e., its energy and angle from the $e, \mu$ or $\tau$, wheress in the QCD calculation the jet rosolution yod is uned. The implication of this effect is that one would tend to measure a lower 3-jet rute (and hence a lower a,value) in heavy quark samples than 
for thowe from light (read mealen) quark.

We have uned computer code provided by E. Muine that allow we to calculate the magnitude of the phase auppression effects for each algoritbm at each yew for $c$ and $b$ quarko[193]. Figures 10.1 and 10.2 show the expected change in the ratios $\alpha F_{3} / F_{3}$ and $R_{3} / R_{3}$ for each of the jet algorithms as a function of vos. The quark mas enters into the jet-rate definition only in terms of the definition for $y_{k}$, The algoritban $E_{t}$ $E O, P$, and P0, than, are effected in an identical manner, vince their definition of $y_{\mathrm{u}}$ are identical". An wrow on ench plot indicates the chowed velue(s) of you. We have assumed the $c(b)$ quark mas is 1.5 (4.75) GeV/c. The dot ted lines on each plot are the resuits for $R_{5} / R_{5}^{*}$ and $R_{3} / F_{5}$ when the quark muetwe are varied by $\pm 0.25 \mathrm{GeV} / \mathrm{c}^{2}$. The suppression factors are aloo listed in Thble 10.1 .

In order to obtein corrected jet rates whase ratius can be related directly to the $O\left(a_{\text {s. }}^{2}\right)$ calculation the 3-jet rate we bave included these corrections in the unfolding procedure, auch that the proper set of exprewions for unfolding the $R_{j}^{j}$ is given by

$$
\begin{aligned}
& n_{2}^{j}=\sum_{j=1}^{3}\left(\varepsilon_{(2-2)}^{i j}\left(1-P^{j} R_{3}^{j}\right)+\varepsilon_{(3-2)}^{i j} P^{j} R_{3}^{j}\right) f^{i} N \\
& n_{3}^{i}=\sum_{j=1}^{3}\left(\varepsilon_{(3 \rightarrow 3)}^{i j} P^{j} R_{3}^{j}+\varepsilon_{(2 \rightarrow 3)}^{i j}\left(1-P^{j} R_{3}^{j}\right)\right) f^{j} N,
\end{aligned}
$$

where $P^{j}$ is the phase-8pece suppression for $R_{3}^{j} / \boldsymbol{R}_{3}^{-l}$. : he values of $P^{j}$ are obtained from those in Table 10.1 with a small amount of algebre, the values of $f^{j}$, and the assumption that $R_{3}^{*}=R_{5}^{4}=R_{5}$. The values of $R_{3}^{j} / R_{5}^{4}$ obtrined with the phasespace-corrected unfolding procudure are given in TaLle 10.1.

\subsection{Obtaining the Values for $\alpha_{s}^{j} / \alpha_{s}^{\text {all }}$}

Now that we have removed any bias due to beavy quark masses, we can continive with the analysis and translate the ratios $R_{3}^{i} / R_{3}^{-4}$ into the denired ratios $\alpha_{5} / a_{0}^{\text {at". }}$. The needed relation is given by combining Equations 8.9 and 8.10 ; eince we have defined $R_{3} \equiv 1-R_{2}$, we include contributions from the calculated rates of 3 . and 


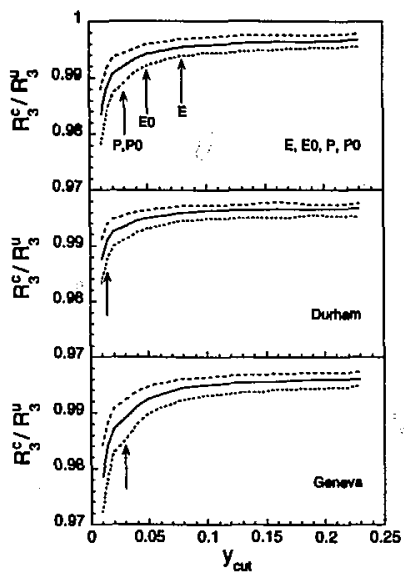

Figure 10.1: The ratio $R_{\mathbf{3}} / R_{3}$ (solid curve) as cklculated using the work of E. Maina. The arrows show the $y_{\text {rat }}$ values used for the analysis. The dashed line above the solid curve is the kame ratio, but for $m_{c}=1.25 \mathrm{GeV} / \mathrm{c}^{3}$. The dashed curve below the solid curve is the same ratio, but for $m_{c}=1.75 \mathrm{GeV} / \mathrm{c}^{2}$. 


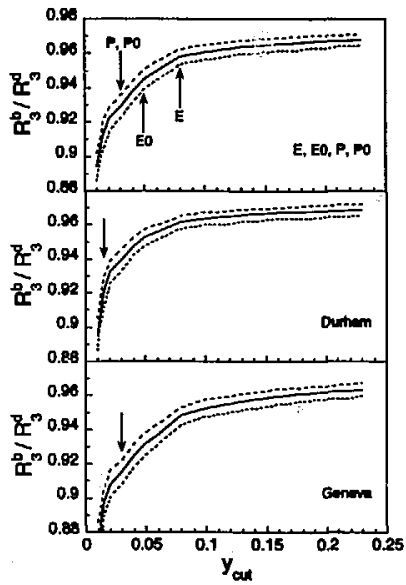

Figure 10.2: The ratio $P_{3}^{b} / F_{3}^{d}$ (solid curve) as calculated using the work of E. Mains. The arrows show the $y_{\text {aw }}$ values used for the andycis. The danhed line above the solid curve is the same ratio, but for $m_{4}=4.5 \mathrm{GeV} / c^{2}$. The dashed curve below the solid curve is the same ratio, but for $m_{4}=5.0 \mathrm{GeV} / c^{2}$. 
Table 10.1: The viluen for $R_{3}^{j} / R_{3}^{\prime \prime}$ derived from applying the phase-spece suppression correction $P^{i}$ wo shows in $E_{q}$ 10.1. The calculated ouppression factors $R_{5}=A_{5} / R_{5}$ and $\mathbf{R}_{5}=R_{\mathbf{3}} / R_{j}$ are also thown.

\begin{tabular}{|c|c|c|c|c|c|}
\hline Algorithm & $R_{3}^{u d o} / R_{3}^{\text {al }}$ & $R_{5} / R_{3}^{\prime l}$ & $R_{3}^{H} / R_{4}^{4 h}$ & $\mathbf{R}_{3}^{\mathbf{a t}}$ & $\mathbf{R}_{3}^{\mathbf{H}}$ \\
\hline $\mathrm{E}$ & $0.934 \pm 0.041$ & $1.200 \pm 0.171$ & $1.006 \pm 0.064$ & 0.995 & 0.958 \\
\hline EO & $0.867 \pm 0.036$ & $1.098 \pm 0.143$ & $1.016 \pm 0.054$ & 0.994 & 0.945 \\
\hline $\mathbf{P}$ & $0.989 \pm 0.027$ & $0.968 \pm 0.107$ & $1.053 \pm 0.043$ & 0.992 & 0.929 \\
\hline P0 & $1.001 \pm 0.026$ & $0.883 \pm 0.100$ & $1.084 \pm 0.041$ & 0.992 & 0.929 \\
\hline D & $0.977 \pm 0.035$ & $1.075 \pm 0.142$ & $0.997 \pm 0.052$ & 0.991 & 0.921 \\
\hline G & $1.017 \pm 0.020$ & $0.924 \pm 0.078$ & $1.005 \pm 0.032$ & 0.989 & 0.915 \\
\hline
\end{tabular}

4-jet production This yields the ratio

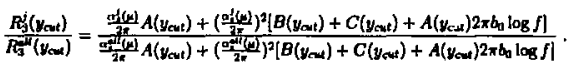

The values of the ration $R_{j}^{j} / R_{j}^{\text {di }}$ from Table 10.1 are input into this equation and it is inverted, yielding the valucs for $\alpha_{,}^{J} / \sigma_{1}^{\text {all }}$ for each jet-finding algorithm. It is worth noting that there is a residual dependence on the vaiue of $\alpha^{-\mu / 1}$ as it appears outsidy of the ratio when this expresston is inverted. (This is easily seen by multiplying numerator and denotninator by $1 /\left(\alpha_{1}^{\alpha}\right)^{2}$.) To handle this correctly, we have used the

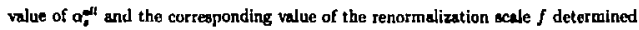
using the $D_{2}$ fit in Ref [131] for each algorithm. These are listed in Table 10.2.

The results lor $\alpha_{0}^{0} / \alpha_{*}^{\text {wll }}$ are given in Table 10.3 along with their statistleal errors. The statistical errors here are the result of the propagation of the statistical errors from the $R_{3}$ ratios obtained in the previous chapter. The results for each algorithm for tach quark flavor do not differ significantly from unity.

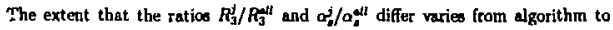
algorithm due to the differences in the coefficients $A, B$, and $C$. The difference in the value of $\alpha_{2}^{2} / \alpha_{a}^{a l f}$ from unity that comes from of inverting Equation 10.3 versus the input difference $R_{3}^{\prime} / R_{3}^{a t}-1$ is shown in Figure 10.3 .

The next chapter will consider the systematic errors on these resulte. 


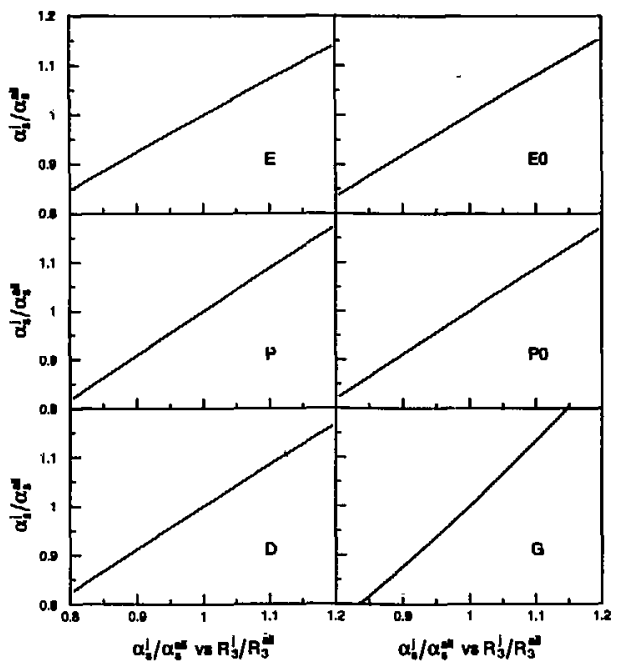

Figure 10.3: Conversion Fuctore for $R_{j}^{j} / R_{\mathrm{s}}$ to $\alpha_{j}^{j} / \alpha_{j}^{-1 /}$ for exch of the $6 \mathrm{jet}$ algorithm. In each plot, the borisontal axis is the input value of $R_{3}^{j} / R_{5}^{\prime \prime}$ and the vertical axie is the resultant value of $a_{0}^{j} / \alpha_{\text {, dil. }}$. 
Table 10.2: The values for $a_{s}^{e l}$, their errors, and the values of $f$ at which they wore derived using the $D_{2}$ distribution in Ref [131].

\begin{tabular}{|l|cc|}
\hline Algotithm & $\alpha_{\text {all }}^{\text {al }}$ & $f$ \\
\hline E & $0.118 \pm 0.012$ & 0.4 \\
E0 & $0.128 \pm 0.021$ & 0.25 \\
P & $0.116 \pm 0.008$ & 0.5 \\
P0 & $0.114 \pm 0.007$ & 0.6 \\
D & $0.125 \pm 0.010$ & 0.5 \\
G & $0.108 \pm 0.005$ & 0.8 \\
\hline
\end{tabular}

Table 10.3: The results of solving Eq. 10.3 for $\alpha_{j}^{j} / \alpha^{\text {all }}$ using the $R_{\mathrm{J}}^{j} / R_{5}^{\text {dl }}$ values from Tabte 10.1. The errors shown are statistical only, but the same events are used by each of the jet-finding algorithms, so the results are highly corzclated.

\begin{tabular}{|c|c|c|c|}
\hline Algorithm & $\alpha_{4}^{2 d /} / \alpha_{r}^{\alpha / 4}$ & $\alpha_{s}^{c} / a_{\varepsilon}^{n d 1}$ & $\alpha_{f}^{6} / \alpha_{f}^{4 / 1}$ \\
\hline $\mathbf{E}$ & $0.954 \pm 0.031$ & $1.154 \pm 0.114$ & $1.015 \pm 0.047$ \\
\hline E0 & $0.974 \pm 0.029$ & $1.081 \pm 0.110$ & $1.019 \pm 0.043$ \\
\hline$\vec{P}$ & $0.991 \pm 0.023$ & $0.974 \pm 0.091$ & $1.047 \pm 0.036$ \\
\hline P0 & $1.002 \pm 0.021$ & $0.901 \pm 0.086$ & $1.073 \pm 0.033$ \\
\hline D & $0.882 \pm 0.028$ & $1.060 \pm 0.111$ & $1.002 \pm 0.041$ \\
\hline G & $1.016 \pm 0.018$ & $0.931 \pm 0.072$ & $1.006 \pm 0.029$ \\
\hline
\end{tabular}




\section{Chapter 11}

\section{Systematic Errors and Consistency Checks}

In this chapter, we consider the potential systematic efiects that could chenge the re-

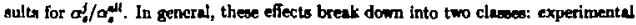
errors and theoretical uncertainties. Experimental errors result from posibie ineccurecies in detector modeling, extra triln on important detector resolution parametera that cannot be constrained by the data, and ertors on the experiment ai meaurenents that function 4 the laput perameters to the madeling of the underlyins phyaica proceoses. The last category includes such things ase errora on the menoured values of the total charged multiplicity in $B$ meson decays, the average $b$ hadron lifetime, etc.. The MC imulations are tuned to reproduce the messured distributions of these quantitien, Bo the poesihle variation in tie experimeatal results must be considered. Theoretical uncertainties include such things os the poesible r'ange of heavy quark masses, the renormalization scale $\mu$, and effects due to different models of hadronization. Each of these and their effect on the final results will be discussent in turn in the sections bolow. We will also consider a number of cross checks that we can perform with the data to guarante that the results are as insensitive a poesible to the specific choice of anaiysis method. 


\subsection{Experimental Errors}

The experitnental systematic urross can be separated into throe categories: those due to uncertainties in the modeling of $b$ hadron decays, those due to uncertainties in modeling $c$ hadron decays, and thoee due to imperfect detector simulation. In general, all of these effects could chenge the clements of the tagsing effiency matrices $\varepsilon$. The influence of the various unecrtaintles on the final results is evaluated in each case by varying the approptiate perenseter in the $M C$, recalculating the matrices $\varepsilon$, performing a new fit to Equation 9.1, and rederiving $\alpha_{a}^{1} / a_{n}^{\text {alt }}$ based on the sante cata sample.

\subsubsection{Event Re-weighting}

Generating a new set of MC events to evaluate the cficets of the variation in each of the parameten governing the production and decay of $c$ and $b$ hadrons would be imposible. Instead, we use an tvent re-weighting scheme to produce the correct distributions in the MC. This works as follows: given notmalizod" dintribution $F(x)$ from the defult $M C$ and the desired nomalized distribution $G(x)$, one can assign 4 meight to each event of $G(x) / F(x)$, where the weight is either calculated analytically using the two distributions (wes is the case in the re-weighting of the lifetimes) or is taken from a look-up table for a given bin of $z$. The totals weights of the eventu are then used to calculate the correct $\varepsilon$ matrices for this set of parameters. All of the re-weighted distributions were checked at the parton-level to insure that they had menn weight of unity and that the normalizations are correct.

\subsubsection{Errors due to b-Hadron Modeling}

As the systcmatic errors duc to the variation of each of the parameters are approxmately the same size for each of the jet-finding algorithms, we have cbosen to merely average tbe results and prescut a single value for each error in the tables. The errors on the ratios $\alpha_{a}^{j} / a_{a}^{a l}$ due to the effects discuosed below nppear in Table 11.1. The are listed here in decressing order of the effect on $\alpha_{j}^{\prime} / \alpha_{a}^{a d}$.

\footnotetext{
- $F(x) d r=1$.
} 
- 6 Hadron Decay Mulliplicity: The mean charged multiplicity in each b-hadron decay is varled by \pm 0.20 tracis, asuming the $b$ hedron decay in each evat hemisphere is uncortelated. The value for the averwse $B$ meson decey multiplicity comes from the $\Upsilon(46)$ measurements of Argus [195], who obtained $\left.\left(n_{\mathrm{e}} h\right\rangle\right)=$ $5.39 \pm 0.15$ tracks. Our variation corresponds to a slightly lerger than $1 \sigma$ chenge, where we have enlarged the crror to allow for sllghtly differint inultiplicities in $B$, and $B$ beryon decays, which are $w$ yet unmesoured. This showld be the largest systematic ecror due to uncertainties in modeling 6 plyysics, as we are relying on the entire spectrum of the number of significant tracks to be properly described by the MC. Obviously, any chenge in the aumber of tracks in $B$ meson decay nust change the calculated taging efficiency.

- o Eragmentation: The muan energy fraction $\left(x_{E}\right)$ received by weakly-decaying $b$ hedrona is varied by $\left\langle x_{E}\right\rangle=0.695 \pm 0.011$, a value consistent with unewurements from LEP[196]. This is done by changing the $\epsilon$ parameter in the Petersen irasmentation function. The tag should aloo be frirly sensitive to the distribution of quark energies, as we have already shown that the bies for tagging 2-jet 6 events is larger than for the other flavrs. This is ensily understooxl, as softer bo produce decay tracks with smaller totul momentum and hence larger extrapoletion errors due to multiple scattering, which makes the track leas libely to miso the IP by a significant amount. Since the $b$ has les boost, it also doesn't travel quite as far before decaying, which also makes that hemizphere herder to tas. Some care must be taken in the re-weighting procedure that is applled in order to calculate the effects of changing the frommeatation. The energies of the $b$ hadrons in events with hard gluon radiation are typically lowor than the mean energy of the distribution and the energies we reducad in a correlated maxner. So, any re-weighting scheme that attempts to weigh each event hemisphere separutely arrive at an improper weight for theme svents. The acturl effects of hemisphere weighting in this case are even more serious, $\approx$ 2-jet events and 3-jet ovents aro shifted in opposite directions when the framentatlon function is cnanged. This is clearly not the desired result of the re-weighting schernol To 
remedy the situation, wn created a routine to calculate an ovent weight based on the vilues of the two most energetic weakly-decaying $b$ hadrons in the event. Larg MC sampies of $b$ events were generuted with diferent Petersen $\varepsilon$ values, and the ratios $a$ he 2-dimenalonal distributions of the two hadron evergies were formed to calculate the mights. Figure 11.1 ahow the sot of meights calculated in this manner for shifting $\left\langle x_{S}\right\rangle$ up or down, as well as the default distribution of badron energies.

- b Hadron Lifetimes: As the easo of tagging b events is mede posedible by the long lifetimes of the $b$ hadron species, any change in the lifetimes will have an eflect on the tagging eficiency. The wlues of the $B$ mean (baryon) lifetimes in the MC sample are 1.55 po $(1.10 \mathrm{po})$. The $B$ meson lifetime was varied by $\pm 0.1 \mathrm{Ds}$, and the $B$ baryon lifetime by $\pm \mathbf{0 . 3} \mathrm{ps}$. Given the small errors on the average $b$ hadron lifetime[194], these crrors may seem a bit generous. However, given the past history of this quantity[199], it is best to be conservative in this particular case.

- b boryon Production Rate: The rate of b baryon production in 6 events was changed from its central value[107] of $9 \%$ by $\pm 3 \%$. A chunge in the $b$ baryon production rate could effect the b-tagging efficiency due to the low lifetime neasured for the baryons.

- $\boldsymbol{R}_{\mathbf{b}}=\mathrm{BR}\left(Z^{0} \rightarrow b \bar{b}\right) / \mathrm{BR}\left(Z^{0} \rightarrow\right.$ hadrons $):$ Without the standard model hadronic branching fractions, this andyais could not have begun. The fraction of b events in $Z^{0}$ decays gives the normalization for the unfolding procedure, so any change in that value has a direct efiect on the values for the other quark Havors, due to the correlntions in the unfolding. The high purity of the b-tag sample largely reduces the effects of these corrclations, howcver. We varied $R_{4}$ by \pm 0.004 about the standard model prediction of $R_{4}=0.220$. This range of error is consistent with the current world average[194].

- $B \rightarrow D^{+}$Fraction: Changes in the fraction of $B \rightarrow D^{+}$decays could have a large effect on the tagging efficiency for $b$ evente, as the long decay path of the 


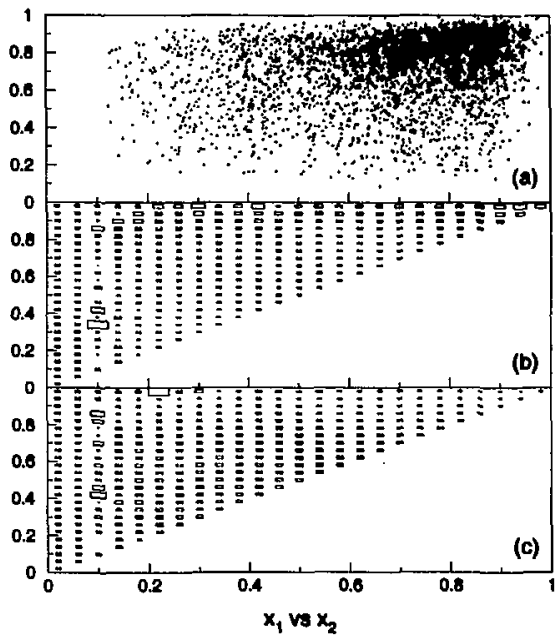

Figure 11.1: (a) The distribution of $x_{1}$ vs. $x_{2}$ for weakly decaying $b$ hadrons, showing the pronounced pealding at higher $x$ values. Figure (b) shows the computed event weights used to simulate $a b$ fragmentation function of Peterson $\epsilon=.0 .003$. The relative size of the boxes in each cell represents the relative magnitude of the ovent weights acrom the weight distribution. Note the weights have the expected dietribution, emphasizing thow events where both quarks have large $x$ values. Figure (c) shows the weights to simulate a b fragmentation function of $\epsilon=, 011$. 
Table 11.1: Syatematic Errors Due to b Hedron Modeling

\begin{tabular}{|c|c|c|c|c|c|}
\hline Source of Ertor: & Central Valuo & Vuriation & $\Delta\left(\frac{a}{a r}\right)$ & $\Delta\left(\frac{a}{a g}\right)$ & $\Delta\left(\frac{\left.g_{0}^{t}\right)}{\left.a_{f}^{t}\right)}\right.$ \\
\hline $\begin{array}{c}B \text { decay multiplicity } \\
B \text { fragmentetion } \\
B \text { meson lifetinto } \\
B \text { baryon lifetitne } \\
B \text { baryon prod. rate } \\
R_{6} \text { (bottom fraction) } \\
B \rightarrow D^{+}+X \text { frection }\end{array}$ & $\begin{array}{c}\left\langle n_{\mathrm{r}_{b}}\right\rangle=5.39 \\
\left\langle x_{b}\right\rangle=0.700 \\
\tau_{b}=1.55 \mathrm{ps} \\
\tau_{\mathrm{H}}=1.10 \mathrm{ps} \\
f_{\Lambda_{b}}=9 \% \\
0.220 \\
0.17\end{array}$ & $\begin{array}{c} \pm 0.20 \mathrm{tks} . \\
\pm 0.011 \\
\pm 0.1 \mathrm{pm} \\
\pm 0.3 \mathrm{ps} \\
\pm 3 \% \\
\pm 0.004 \\
\pm 0.07\end{array}$ & $\begin{array}{l}0.005 \\
0.003 \\
0.005 \\
0.002 \\
0.009 \\
0.002 \\
0.002\end{array}$ & $\begin{array}{l}0.046 \\
0.010 \\
0.030 \\
0.012 \\
0.013 \\
0.003 \\
0.005\end{array}$ & $\begin{array}{l}0.023 \\
0.016 \\
0.012 \\
0.004 \\
0.007 \\
0.006 \\
0.001\end{array}$ \\
\hline
\end{tabular}

$D^{+}$dowtstrenm of the $B$ meson decay point enhunces the efficiency for tagging that event. The uncertainty of this branching fraction is[194] $\pm 4 \%$ eurrently; we sosign a $\pm 6 \%$ error to acrount for the uncertaintios in the production rates of all of the charmed hadrons in $B$ decays.

\subsubsection{Errors due to $c$-Hadron Modeling}

The errors on the ratios $\alpha_{j}^{j} / a_{a}^{a l l}$ due to the effects discused below tippear in Table 11.2. The ere listed here in decreasing order of the effect on $\alpha_{*}^{c} / \alpha_{*}^{\alpha, 2}$.

- $R_{c}=\mathrm{BR}\left(Z^{0} \rightarrow \mathrm{x}\right) / \mathrm{BI}\left(Z^{0} \rightarrow\right.$ hadrons $):$ As the c-tug in of relatively low purity and contaninated by both uds and $b$ events, the fraction of $c$ ivents asumed for thet sample has a large effect on tho outcome for $a_{f} / a_{a}^{a}$. This is due to the large background nubtraction that occurs to derive the 3-jet rate for charm; the effect is shown graphically in Figure 11,2, where the value for $R_{C}$ input to the analysis is saried, and the reaults for $\alpha_{s}^{j} / a_{\text {all }}^{\text {ef }}$ for all three flavurs are plotted. As the amount of charm in the sanple is woumed to be leas and leas, the 3-jet rates for $u d s$ and $b$ adjust to maintain the total 3 -jet rate in the charm tas sample, though the $x^{2}$ of the fit (not shown) does gei worse. We varied $R_{c}$ by \pm 0.016 about the tandard model value of 0.170 . The veritution is alghtly 


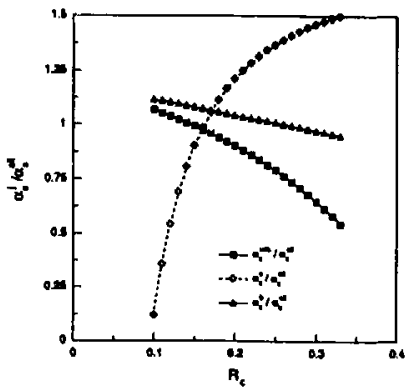

Figure 11.2: The reaultu for $\alpha, / \circ$ dit when the input vilue of $R_{c}$ in varied. The excen branching fraction is ascumed in this case to come from wds events.

larger thas the error on the world-average|194] for $\boldsymbol{R}_{\mathrm{c}}$. The central value of the world average lo approximately $1.5 \sigma$ low at thl point, at $\boldsymbol{R}_{c}=0.156$. Since the measurements are atill consistent with the standard model, we choowe to use that central value.

- c fragmentation The nean energy fraction $\left\langle I_{s}\right\rangle$ received by weakly-decuying a hadrons was varied (in the same manner as the $b$ fragmentation case, above) by 〈Is $\div 0.50: \pm 0.012,2$ wlue consistent with messurements from LEP[198].

- $\bar{a} \rightarrow D^{2}$ Fraction: Once agan, due to the long $D^{+}$lifetime, we would expect that the froction of charm decays containins tignificant trecke depends on the rate of $D^{+}$production in $\propto \bar{x}$ events. Wo soign $a \pm 5 \%$ error to scount for the uncertajatics in the production rates of all of the chermed hadrons. 
Table 11.2: Systematic Errors Due to c Hadron Modeling

\begin{tabular}{|c|c|c|c|c|c|}
\hline Source of Error: & Central Valua & Vuriution & 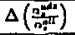 & $\Delta\left(\frac{a^{c}}{a^{a}}\right)$ & $\Delta\left(\frac{a^{b}}{n^{h}}\right)$ \\
\hline $\begin{array}{l}R_{c} \text { (charm fraction) } \\
c \text { fragmentation } \\
c x \rightarrow D^{+}+X \text { fraction } \\
c \text { decay multiplicity }\end{array}$ & $\begin{array}{c}0.170 \\
\left(I_{c}\right\rangle=0.494 \\
0.2 .0 \\
\left\langle n_{c h}\right\rangle \sim 2.34\end{array}$ & $\begin{array}{c} \pm 0.017 \\
\pm 0.012 \\
\pm 0.04 \\
\pm 0.20 \text { tks. }\end{array}$ & $\begin{array}{l}0.016 \\
0.004 \\
0.003 \\
0.003\end{array}$ & $\begin{array}{l}+0.05 \\
0.005 \\
0.016 \\
0.01 \\
0.0008\end{array}$ & $\begin{array}{l}0.008 \\
0.003 \\
0.002 \\
0.006\end{array}$ \\
\hline
\end{tabular}

- c Derry Multiplicity: The mean decay multiplicities of the $D^{0}, D^{+}, D_{\Delta}$, and $\Lambda_{c}$ are varied by $\pm 0.06, \pm 0.10, \pm 0.31, \pm 0.40$, respectively. The measurements of these $c_{\text {..cay }}$ inultiplicities are from MARK-11t[200]. The multiplicities for $D^{0}$ and $D_{a}$ in our MC agree exactly with the uncesured valucs, so the variations ase just the experimental errors. For the $D^{+}$, our MC multiplicity distrihution is deficient in 1-prong decay nodes when compared with MARK-III's mensurement. So, the variation for this decay mode is the difference in the mean decay multiplicity between our MC value and the messurement. The varistion for $\Lambda_{c}$ is estimated from varying the parameters that can effect charm baryon production in the $M C$.

\section{1,1,4 Other Experimental Errore}

The errors on tho ratioe $a_{a}^{j} / a_{a}^{\text {all }}$ due to the effects discusend below appear in Table 11.4 .

- Limited MC Statistics: Since the $c$ efficiency matrices are evaluated using the MC, each matrix element has a statistical error duc to the finite number of MC events available. We estimated the effects of this uncertainty in the tags by generating the multinomial distribution for the elements of each column of each $\varepsilon$ matrix based on the MC statbstics used to create the matrix. This was repeated 1000 times for each algorithm, with results for $\alpha_{s}^{j} / \alpha_{\text {, }}^{\text {"l derived each }}$ time using the numbers of tagged events in the data. The atandard deviations 
of the $\alpha_{n}^{j} / \alpha_{a}^{\text {alt }}$ distributions were taken as the error due to finite statiaties. This procediure results in errors that are approximately what one expects beed on the total number of MC arri' data events.

- Tracking Efficfency: Any variation of the number of tracts avalleble for we in tagging will have some eflect on the tag efficiency. As diecused in detail in Section 7.1, the average multiplicity for togsing tracks difers between MC and dats. We have applied a correction to the MC to remove this diecreparay, and have assigned a conservative error of \pm 0.3 tracks on the correction. The vuriation in the final results due to changing this correction are relatively wall.

- Tails in the IP Position Distribution: If the position of the IP in incomsetly deternined, then it is powible that a large niumber of tracks in any given event could mis the IP by a significant amount and cause the owent to be included in the $b$ tas ample. Since we cannot rule sut the preacnce of $a 100 \mu \mathrm{m}$ tell at the level of $0.25 \%$, wo add this amount of teil to the MO events to simulate this possible effect.

- Tracking Resalution We discused in Chapter 5 the "unamearing' correction which was applied to tracks in the MC to remove errors in vimulating the CDC positions. We take here the full correction a a syntematic otror and perform the analysis without tho unsmearing applied. This turns out to be a small effect. The effects of changing the efective tracking resolution by requiring two VXD hits on each track were dso minimal, lending us to believe that the tracking is well modeled by tho MC.

- "Vee"-Finding Wo can place an upper limit on the possible eftects of a variation in the frequency of $s \bar{j}$ production in hadronic shomers by allowing $K^{0}$ and $\Lambda$ decays intc our sample of charged track. Even though the efficiency for reconstrueting their decays becomes stnall in the region claw to the IP where they could be more ensily mlataken for a heary quark decay, the frection of tracks from these sources that remains in the analysia when th ey aro not excluded changer by far more than the $10 \%$ variation ane can atimate for the 
uscerteinty on si production. The overall effect is aloo small.

- Voriation of Event and Thack Selection Cuts: Chenging the event and track selection cuts checka the valjdity of the MC simulation of the detector response, and, to $a$ lemer extent, tho physics included in the $c$ and $b$ hadron decay modeis. As we heve alrady considered the systematic errors due to changing the physics perametern, we will tcy not to double-count eources of error, choosing for consideration cuis that should be relatively independent of those parmeters". The effect of changing these cuts is to modify the total efficiency for an event to pass the crent seluction cuts; since the effieiencies for everts of each quark flavor to pere the cuts aro virtually identica, wo would expect these effects to be sunll. Due to the sulall size of these effects relative to the statiatical errors (and fluctuations) on the measured values, we varied each of the cuts in ateps away fron the central value and used the slope derived from these points to

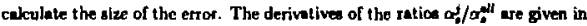
Table 11.3. Due to the small number of events that are affected by the andysis chenges, all of theee slopes differ from eno by lew then 0.10 ; the eflecto of these variations on the analysts will be ignored.

Thrust Axis Containment: the cut on $\left|\cos \theta_{\text {Thumen }}\right|$ was varied over $0.67 \leq$ Cut $\left(\left|\cos \theta_{\text {Thrum }}\right|\right) \leq 0.75$, a range conoistent with our estimates of a 2 smearing in the thrust axis dirextion due to shower Huctuations in the calorimeter reaponse.

Visible Energy: the minimurn visible energy required for an event to be selected we varied by $16 \mathrm{GeV} \leq C u\left(E_{\text {vog }}\right) \leq 20 \mathrm{GeV}$. The effect of this variation is to subtly modify the probabilities for a parton-level event to switch asaignmeats between a 2-jet eveat and \& 3-jet event aud to change the probability of a parton-level 3-jet event to pas the exloction cuta.

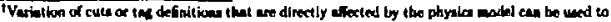

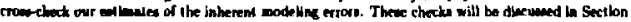
11.3.
} 
Tuble 11.3: The derivatives of $r^{\prime}=\alpha_{3}^{j} / \alpha_{2}^{4 *}$ with reapect to the veriod andyats parameter $x$.

\begin{tabular}{|c|c|c|c|c|c|}
\hline Source: & Central Value & Variation & $\partial r^{20} / \partial x$ & $\theta+2 / \theta x$ & $\partial r^{*} / \partial z$ \\
\hline $\begin{array}{c}E_{\text {wts }} \\
\cos \theta_{T} \\
n_{t+k} \\
\cos \hat{\theta}_{t r k} \\
p_{t r y} \\
p_{t}\end{array}$ & $\begin{array}{c}E_{\mathrm{win}}>18 \mathrm{GeV} \\
\left|\cos \theta_{T}\right|<0.71 \\
n_{\text {Th }} \geq 7 \\
\left|\cos \theta_{\text {TRA }}\right|<0.8 \\
p_{t a s} \geq 0.5 \mathrm{MeV} \\
p_{1} \geq 0.2 \mathrm{MeV}\end{array}$ & $\begin{array}{c} \pm 2 \mathrm{GeV} \\
\pm 0.04 \\
\pm 1 \\
\pm 0.04 \\
\pm 0.1 \\
\pm 0.1\end{array}$ & $\begin{array}{c}<.01 / \mathrm{GeV} \\
0.805 \\
0.003 \\
-0.310 \\
-0.152 / \mathrm{GeV} \\
0.074 / \mathrm{GeV}\end{array}$ & $\begin{array}{c}<.01 / \mathrm{GeV} \\
-3.112 \\
0.031 \\
0.248 \\
0.083 / \mathrm{GeV} \\
-0.388 / \mathrm{GeV}\end{array}$ & $\begin{array}{c}<.01 / \mathrm{GeV} \\
0.987 \\
0.017 \\
1.185 \\
0.265 / \mathrm{GeV} \\
0.065 / \mathrm{GeV}\end{array}$ \\
\hline
\end{tabular}

Charged Track Multiplicity: the number of charged tracks required for an event to pen the eeloction cute was variod between 6 and 8 treckit to check the effects of allowing sorpe variation in the exoount of backgrotund in the emmpic.

Charged Track Definition: The defintion of the allowed track parameters for a track to be included in the total charged multiplicity of an event wo varied within remonable bound to atudy the effectu of additional sol tracks on jet definitions, totel visible energy, and the beckgrounds in the enmple. The allowed $p_{\perp}$ was varied between $100 \mathrm{MeV} / c$ and $300 \mathrm{MeV} / c$. The allowed track | $\cos 0 \mid$ wre varied between 0.76 and 0.84 . Variation of the slowed track docan and doca, produced negligible changes.

- Tagging Thet Homentum Cut: The poulble variations in the $\varepsilon$ matrices due to the uncertainties in modeling the momentum epectre of the $b$ and $c$ hadron decay products wes not explicitly considesed above is the physics modeling enrons. We cun eatimate the effect on this analysis by varying the allowed mornenturn of the tagging track uned in the andyais. Sioce the minimum momentum cut is already set to the relatively bigh value of $0.5 \mathrm{GeV} / c$, we should be insensitive to the low-momentum repon where mot of the uncersinty lies. A variation of $\pm 100 \mathrm{MeV} / \mathrm{c}$ in this cut gives the expected anell effects on the find result. 
Table 11.4: Syxtematic Erroix Due to Detector Modeling

\begin{tabular}{|c|c|c|c|c|}
\hline Sourc & Veriation & 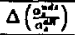 & $\Delta\left(\frac{a_{0}^{f}}{a_{f}^{\prime}}\right)$ & $\Delta\left(\frac{a^{b}}{a^{d}}\right)$ \\
\hline $\begin{array}{l}\text { M.C. Statiatics } \\
\text { tracking efficiency } \\
\text { IP Powition Trils } \\
\text { tracking resolution } \\
\text { Vec finding }\end{array}$ & $\begin{array}{c} \pm 0.3 \text { tracks } \\
0.25 \% 100 \mathrm{\mu m} \\
\text { no ursimear } \\
\text { none }\end{array}$ & $\begin{array}{l}0.011 \\
0.003 \\
\sim 0.001 \\
<0.001 \\
<0.001\end{array}$ & $\begin{array}{c}0.048 \\
0.031 \\
\sim 0.005 \\
0.017 \\
\mathbf{0 . 0 1 3}\end{array}$ & $\begin{array}{c}0.014 \\
0.017 \\
\sim 0.005 \\
0.014 \\
0.007\end{array}$ \\
\hline
\end{tabular}

\subsection{Theoretical Uncertainties}

Uncertainties in the underatanding of the exact phyvical prosese uncierlying the production and decay of hadrons and the emiasion of hurd gluou adiation can lead to varying prediction for the theoretical inputs to our models of hedronic interactions. Some phynical paraneters, such ws the puuric maseses and the rate for gluon sphitting to hesvy quark pairs, have only weak experimental constraints. The sheer difficulty of calculation in perturbative QCD and the size of $a$, result in uncertainties in the surrent calculations due to the effect of as-yet-uncalculated higher-order processes. The MC simulations that reproduce the observed data have tunable parameters that are not directly observable experimentally and are thu allowed to vary over bome range. All of these elements contribute to the theoretical uncertainties associnted with this analysis. Again, we present in Table 11.5 for cach of the quark flavora an sverage of the systemat:c c.rors for all of the jet-finding algorithur.

- Hadronization Modaluns We checked the variation of the parton-level 3-jot rates as functions of the various parameters within JETSET 6.3 that control the at ring fragmentation process. Their effects on the final results were then eviluated by changing the 3 -jet rates in the MC by the variation seen in changing the parametern. Since the ratio $\alpha_{j}^{j} / \alpha_{1}^{\text {dit }}$ is not sensitive to the absolute 3-jet rate in the $\mathrm{MC}_{\mathrm{t}}$ the effects of these variations nfe negligible. The paraneters atudied included

$Q_{u}(\operatorname{PARE}(22))$, which controls the parton shower cut-of $\left(Q_{0}=1.0_{-0.5}^{+1.0} \mathrm{GeV}\right)$ 
$\sigma_{n}$ (PAR(12)), which varies the width of the gaumian dimeribution of traseverue momentum senerated in the perton mower relative to the oridinal queck direction $\left(\sigma_{1}=0.39 \pm 0.04 \mathrm{GeV}\right)$

$a$ and $b$ (PAR(31) and PAR(32)), the coefficiente in the aymmetric Lund fragmentation function (theac were checked at the hadron-level)

- The rate for $g \rightarrow$ QQ: $A$ lurge rate for gluons to split into heavy quarla could change the frequency with which uds events are tagsed by the henvy flavor tere. As there are very few experibental contraints on thin rate, wo beve eatimated the effect by varying the rate for $g \rightarrow Q Q$ by $\pm 50 \%$ from the JETSET defoult value. This has a relatively emall effect on the roults.

- The Uncertainty on afl; As mentioned in Section 10.2, the results for the $a_{\text {, }}$ ration depend on the value of $\alpha_{4}^{\prime \prime}$ uwed when converting from jet rates to $\alpha_{4}$ weing

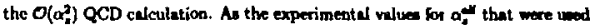
also have errors aseociated with them, their variation munt sio be coneidered as a source of uncertainty. Thix in included at a theoretical uncertajnty becave the dominant error on the value for $\alpha_{\infty}^{*}$ is due to the inability to fix the value of the remormalization wale $f=\mu^{2} / \Delta$, which arimes from a cut-of put into the theuretical calculatione to avoid the need to calculated higher-order processes. Thus, to extirnate the efrects of this error correctly, we need to vary

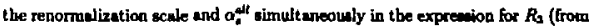
Section 8.3):

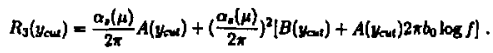

This generates up to $21 \%$ exror on $\alpha_{j}^{j} / \alpha_{3}^{24}$, which is fer smaller than the $10 \%$ error this effect hw on the sbeolute determination of $a_{1}$ " from the $D_{2}$ distribution][131]. The variation of ewch of the value of $a_{a}^{-1 /}$ for the individual jet algorithnu (see Table 10.2) was und to atimute tbe error.

- Uncertunties in the $R_{4}$ Cakulation: As diecumed in Section 8.3, the calculated 4-jet rate doss not match that obeerved in the data by approximately a fector 
of two for all algorithms except for $G$. This is presumably due to uncalculated coefficients of $\mathcal{O}\left(a_{a}^{3}\right)$ and higher. We take the full $100 \%$ variation in the value of

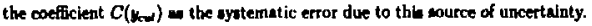
It hes an almoat negligibly unall effoct on the find resulus.

- Variation of the Hervy Quent Masses: The correction applied in the previous chapter to account for the smaller phee-space for hard gluon emingion in heavy quark events reliex on knowing the values of the $b$ and $c$ puark manes. As this

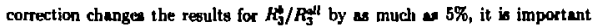
to consider poesibin varintions in its inagnitude. The quark manes uned in calculating the centrel values of the corrections were $4.5 \mathrm{GeV} / \mathrm{c}^{2}\left(1.5 \mathrm{GeV} / \mathrm{c}^{2}\right)$ for the $b\langle c\rangle$ quark naes, and we considered variations of $\pm 0.25 \mathrm{GeV} / \mathrm{e}^{2}$ about these values to estiniate the error on the parton-level quark masees uned in the calculation.

- Uncalculata' Higher-Orders in the Phase-Space Suppresvion Calculation: The calculation upon which our phase-sp]nce corrcetion is bued[193] hen only been perforned to leading order in $\alpha_{\text {.- }}$ Wo have as yet mule no eatimate of the possible size of the next-to-leading term; they should be of order $a_{3} / \pi$ times the first order correction, which would make them enall.

- Jet-Algorithmic Dependenx of Reswlo: All of the reoults quoted in this chepter have been the sverages of the shifts in the values for $\alpha_{3}^{3} / \alpha$, due to the variations of the different parameters which can affect the results. We have chosen to take a simple mean of thcse resulus, as there is no rewon a priori to prefer one algorithm over any other. However, there is significant scatter in the final values, which is posibly not all statistical. Since all of the jet algarithms are being uned to perform the same measurements on the sume dats wet, and the results should be independent of jet-algorithm to finet order, we attribute the scatter to uncalculated higher-order terms that contribute to the 3-jet rate. To extimate the aize of this effect, we calculate the r.m.t. of the results for each flavor relative to the mean value, and quote this as the theoretical uncertainty amociated with 
Table 11.5: Theoretical Uncertaintios

\begin{tabular}{|c|c|c|c|c|c|}
\hline Source of Error: & Central Value & Variation & $\Delta\left(\frac{4}{4}\right)$ & $\Delta\left(\frac{\alpha}{z}\right)$ & $\Delta\left(\frac{2}{2}\right)$ \\
\hline $\begin{array}{c}\text { Hedronization } \\
g \rightarrow Q Q \text { rate } \\
a_{g} \\
\Delta C\left(y_{c}\right) \\
\Delta m_{b} \\
\Delta m_{c} \\
\text { Jet } A l_{g o r i t h m}\end{array}$ & $\begin{array}{l}4.5 \mathrm{GeV} / \mathrm{c}^{2} \\
1.5 \mathrm{GeV} / \mathrm{c}^{2}\end{array}$ & $\begin{array}{c} \pm 50 \% \\
\pm .25 \mathrm{GeV} / \mathrm{c}^{2} \\
\pm .25 \mathrm{GeV} / \mathrm{c}^{2}\end{array}$ & $\begin{array}{c}0.002 \\
0.001 \\
0.003 \\
0.001 \\
0.001 \\
<0.001 \\
0.021\end{array}$ & $\begin{array}{l}0.007 \\
0.002 \\
0.009 \\
0.003 \\
0.002 \\
0.002 \\
0.096\end{array}$ & $\begin{array}{l}<0.001 \\
0.002 \\
0.010 \\
0.003 \\
0.004 \\
0.001 \\
0.027\end{array}$ \\
\hline
\end{tabular}

Tublo 11.6: Summary Table of Syuternatic Erons

\begin{tabular}{|c|c|c|c|}
\hline Sonrce of Error: & $\Delta\left(\frac{0}{6}\right)$ & $\Delta\left(\frac{6}{m}\right)$ & $\Delta\left(\frac{o_{0}^{k}}{a_{n}}\right)$ \\
\hline $\begin{array}{c}\text { Expurimental Erroct: } \\
\text { B Phyrica } \\
\text { C Physic } \\
\text { Detector Modeling } \\
\text { MC Statietica }\end{array}$ & $\begin{array}{c}0.0084 \\
0.017 \\
0.003 \\
0.011\end{array}$ & $\begin{array}{l}0.060 \\
0.060 \\
0.032 \\
0.048\end{array}$ & $\begin{array}{l}0.033 \\
0.011 \\
0.017 \\
0.014\end{array}$ \\
\hline $\begin{array}{l}\text { QCD Theoreticel Uncertainty } \\
\text { Jet Algorithm }\end{array}$ & $\begin{array}{l}0.003 \\
0.021\end{array}$ & $\begin{array}{l}0.011 \\
0.097\end{array}$ & $\begin{array}{l}0.012 \\
0.028\end{array}$ \\
\hline
\end{tabular}

the QCD calculations dewcribing the jet-finding algorithms. This is by far the largeat theory error.

All of the ayntematic enrors are summarized in Table 11.6, where the total contribution of each category to the error is listed.

\subsection{Checks on the Validity of the Results}

In this sectlon we present two cros-checks that cive us confidence that the analynis method wo have uned and our eatimates of the anociated uncertuinties aro robust. 


\subsubsection{Variation of the Chowen yeut}

To insure that the dependence of our results on the choice of year wes minimal, we repeated the entice enalysis at different yau values for all of the jet algoxittums. The average values are shown in Figure 11.3 and 11.4. Note that all of the points are highly correlated. No significent deviation from our resultu is obverved.

\subsubsection{Variation of the Tag Definition}

We defined as a significant track one that misses the IP by more than $3 \sigma$. We can change this requirement and obmorve the variation in our restults. This serves to verify that our mtimates of the pbyzics and tracking modeling errors are corroct, since differences betwen the true paraneters and our MC simulation could manifest thenselves in a dependence of the result on what is chosen for the sigtificance cut. Figure li.s shows the results for $\alpha_{,}^{\prime} / \alpha_{*}^{\prime \prime}$ as the gignificant cut is varied. There seems to be a systematic slope in all of the results. It is interenting to consider this result for the Veto perlod colapared with the Pre and Non-Veto periods, which is alown in Figare 11.6. It can be seen that virtually all of the variation with respect to the significance cut occurs in the Velo period. As the only substantial difference in the MC cimulation between the Veto period and the others is in the effects of the track trigget, we ent only surmise that improper simulation of this effect is the root cause of this dependence. To check tliks, we analyzed the Pre-Veto period data with the Veto period MC; without applying the trigger veto to the MC, and compared these results with the sane analysis performed with the correct $\mathrm{MC}$ and dats match. The reaults are consintent, which implies thut nothing is wong with the MC simulation excluding the sinulation of the trigger veto. We can check th, validity of the veto simulation in two ways. First, we cen njessure the branching fraction for $Z^{0} \rightarrow b \bar{b}$ in the Preveto and Veto periods reparately, and ace if they compare. Using the MC veto simulation to correct for the fact that $b$ events during the Veto period are lesw likely to pas the trigger, we oltain $A f^{\nu}=0.233 \pm 0.007$ and $R_{b}^{V}=0.226 \pm 0.006$, where the errors art ot at istical only. The first value is in excellent agteement with

\footnotetext{
ISese Tatic 6.2 .
} 


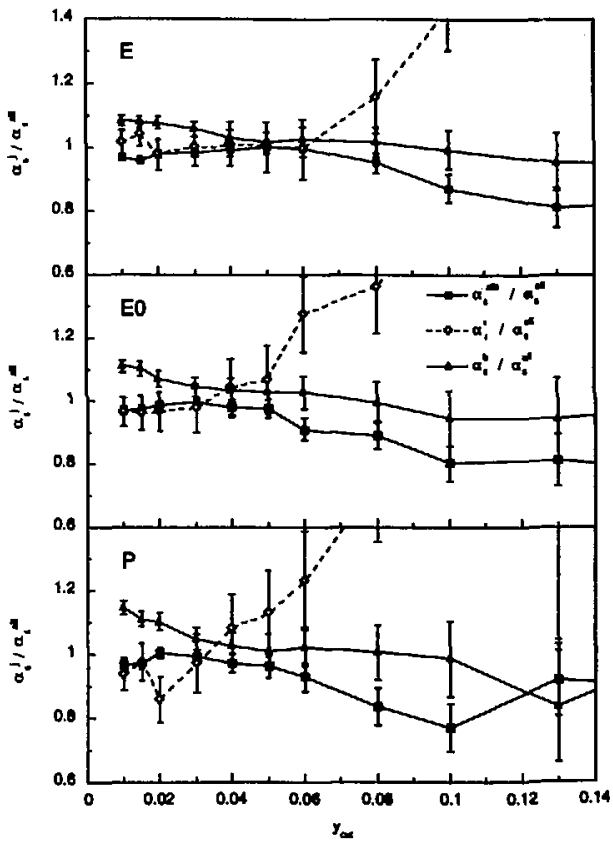

Figure 11.3: The Reults vs. You Choice for $E$, EO, and P 


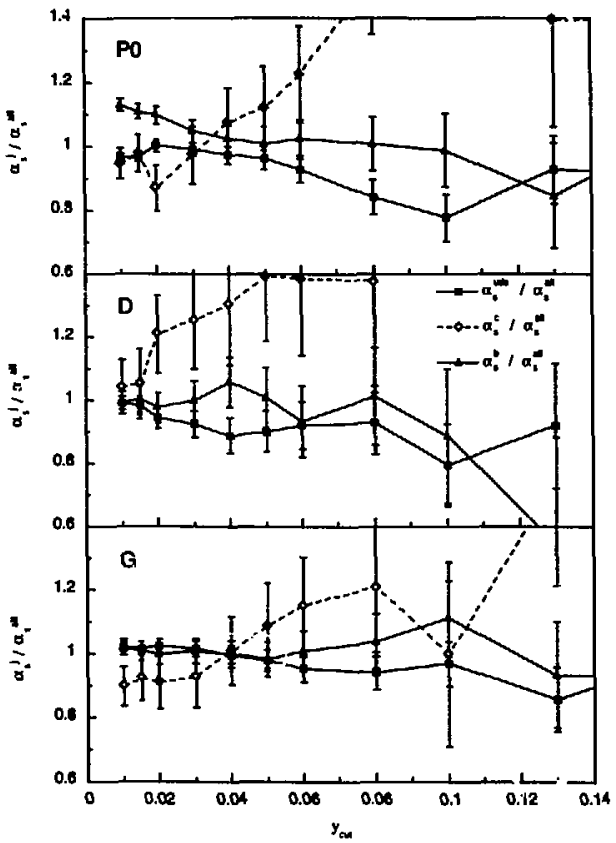

Figure 11.4: The Reaults us, you Choice for P0, D, and G 


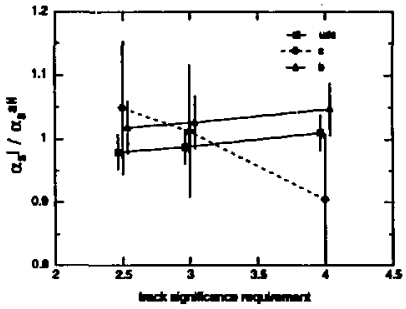

Figure 11.5: The results of the andyais obtained for diferent cuts on the track sif-

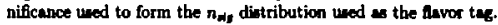

that of the official SLD $R_{4}$ mearurement[182] of $R_{4}=0.231 \pm 0.004$ ueing the shme technique and the Preveto and Nonveto data samples. Since the values for the two run periode are within $1 \sigma$, we cannot measure any difference introduced by the veto simulation using this technique. Another method is to vary the rate of the veto itself. We conidered the Veto period with a tas requirement of $b_{\text {norm }}>4.0$, as this is where the maximum deviation occure in Figure 11.6. No shift in the values of the three

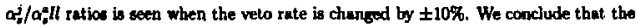
small slope seen on this plot is dus to the statistics of the impact parameter tas, and not to some systematic problen with the veto simulation.

\subsubsection{Sensitivity to Anomalous Flavor-Dependent Strons Couplings}

To invertigate the eensitivity of this analyais method, we ued a subample of the MC a a mock data cample and removed different fractione of 3-jet bor uds and $c$ events 20 as to vary $R_{5}$. This mock data sample was then subjected to the standard 


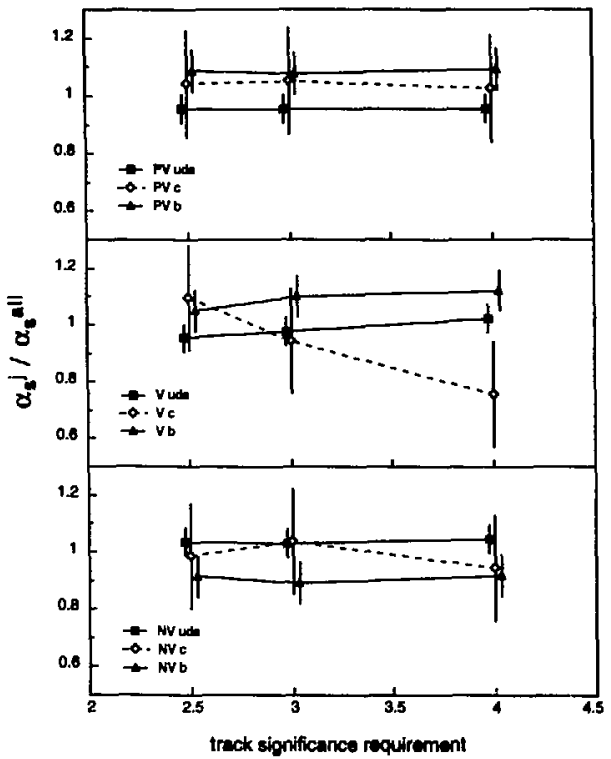

Figure 11.6: The results of the enalysis obtained for different cuts on the track significance used to form the $n_{\text {sig }}$ distribution ueed as the flavor tag. The three different run periods are shown for comparison. 


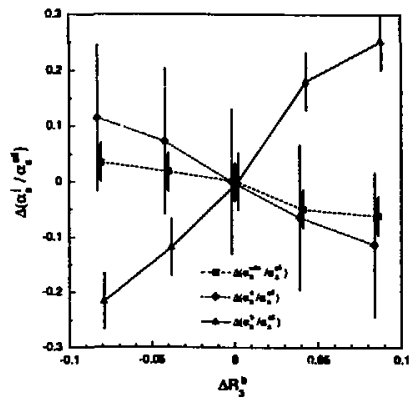

Figure 11.7: Sensitivity to Input Anormolous Strong Coupling

analysis. The results are shown in Figure 11.7. One can see that if there were large flavor-dependent differences in the strong coupling we would have oberved them in this analysis.

We now move on to the presentation of the final results, and some comments and conclusions about this analysis. 


\section{Chapter 12}

\section{The Flavor Independence of $\alpha_{s}$ : Conclusions}

\subsection{Final Results and Discussion}

The finel results fot all of the jet algorithms are showa in Figure 12.1. For the avernge values stown an this figure, the c.m.s. of the results over all algorithns is added in quedrature to the other theoretical uncertainties, as was discused in Section 11.2. The everage values, including this additionul error, ase:

$$
\begin{aligned}
& \frac{\alpha_{*}^{2 t}}{\alpha_{*}^{n t}}=0.987 \pm 0.027 \text { (stat) } \pm 0.022 \text { (syst) } \pm 0.022 \text { (theory) } \\
& \frac{\alpha_{s}^{e}}{\alpha_{s}^{*}}=1.012 \pm 0.104 \text { (atat) } \pm 0.1 \mathrm{~m} \text { (oyst) } \pm 0.096 \text { (theory) }
\end{aligned}
$$

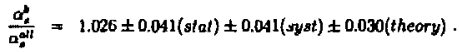

As a firat point of diecussion, it is clear from the above results that $a_{\text {, is indepen- }}$ dent of quark Havor within our present experimental sensitivity. Our novel means of eparating the different quark flasors irclusively by differences in the lifetime content of the charged tracks has prover to be free of large systematic bives while allowing us tos nalke use of the full statistica of our data umple. In particular, the measurement 


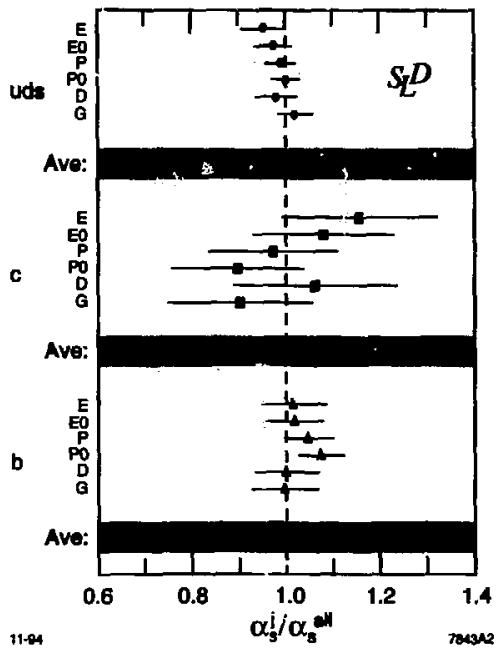

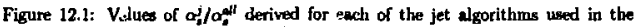
andynis for each of the quark favors (see text). The eror bars on the average values include the at atistical and systematic errors and the total theoretical uncertainty.

of ratioe ba minimized the effects of the $\mathrm{r}$ ormalization scale uncertainty and the

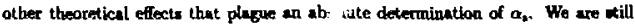
menitive to effects relating to the different et algorithms. The high purity of twe 
uds and $b$ taga results in smaller total errors for these two results due to teduced senuitivity to fuctuations in the backgrounds wlich are belng subtracted in tlie unfolding procodure. The reault for charm, on the other hand, sufiens froun baving a Agna-to-noine ratio of 0.5 ; nny smell change in the background composition leads to lage excursions of the extracted value for $\alpha_{j}^{c} / \alpha_{a}^{a}$. This is the source of the large syotematic and theoretical errors on the charm result. Possible improvements on this method will be discuraed below.

It is interesting to note the large theoretical uncertainty, which is dominated by the different values obtained for the $a$,ratios when using the six jet algorithms. As mentioned in the previous chapter, this is most likcly due to uncalculated bigher-order terms in the theoretical predictions for the jet rates. This large variation of the rcsults was expected from previous work[13l] on measurements of $\alpha_{A}$, but this is the first time a sybtematic study of this effect has been done checking the behaviour of the jet algorithms for quarks of flavors (masses). The possible variation of different observables in measuring the ratios $\alpha_{n}^{j} / \alpha_{s}^{\text {aff }}$ and the associated theoretical uncertainties have been largely ignored in ull of the other experimental results on fiavor independence.

To compare our limits on flavor independence with thowe obtained by other experimental nethods, we show in Figure 12.2 the above results along with thoce from the LEP experiments * discussed in Chapter 3 . As the only other andysis which has attempted to measure the strong coupling to each quark flavor independently is the one fram OPAL[145), it will be the focus of much of the compersative discussion. It should be mentioned that the errors on the measured ratios are necessarily enlarged by loosening the asomption that all of the quark Hevors have the same strong coupling except the one under study; both our rwuits and the ones from the OPAL study share this consequence of a more general analysis.

The advantages of our method over those used by OPAL for the light quark tegs is immediately obvious, as our results have smaller errors even though the analysis is based on a factor of $\mathbf{2 0}$ leas data. This is inostly due to the use of the precision tracking and our knowledge of the stable IP position as a method of excluding heavy quark wints from the sample of selected events, rather than selecting light quark events by

- The TASSO noulle have ban superacded in precision nnd will not be cansidared hre. 


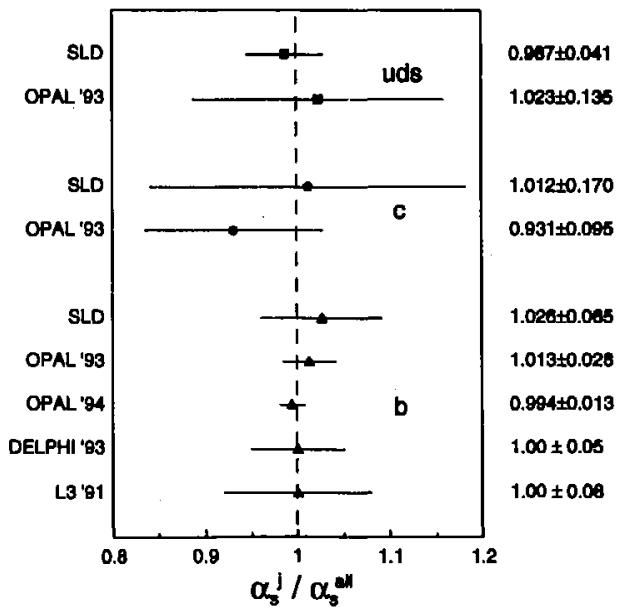

Figure 12.2: Valucs of $\alpha_{j}^{j} / \alpha_{d}^{\text {ell }}$ mensured in this thesis compared with other experimental results. References for other values are provided in Chapter 3. For a discussion of results, seo text. 
some idnematic method from anple with large, relntively unknown beckground. Even with the somewhat larget errors on the result for cherm, our errora are more of lew comparable to those obteined by OPAL due to the ineficiency of their chum teg (reconstructing $D$ mesons) and its larger systematic bias. For b quarks, larger atatiotica and the amaller systematic etrors anociated with s bigh-p lepton tag produce - more precise reault from OPAL.

In contrast, the advantages of ubtaining a high statistics high purity sample and using that as asting ground are exemplified in the newcat reault from OPAL, (46] for $0_{*} / a_{*}^{\prime \prime}$. Although they feil to consider properly the additional theoretical error absociated with combining correlnted mesurements of the same quantities, thoir result is still by far the most precise tent of flavor independence in the strong interection.

\subsection{Possible Improvements of the Method}

The primary weaknesses of the method used in this andyzis are the low purty of the charm sample and the reliance on the Monte Carlo sinulation of heavy quark decays to represent the true decay propertics. The latter introduce the largeat aystematic errors, while the former serve to magnify ofl of the errors asoociated with $\alpha_{n}^{c} / \alpha_{n}^{N}$. Systematic erross due to uneortaintics in the heavy quark decay model can be drestically reduced by performing a "double tag" using both hemispheres in the event to actually measure the tagging efficiency for a given tag'. Given the stability of the SLC beam spot, a technique of this sort could also be used in traging the light flavors instead of just $b$ events. A more complicated version of the elficiency metricen used in the unfolding could be created that accounts for the flavor composition of doublytagged vs. mixed-tag events. Since the tag efficiency could then be messured for uds and $b$ events, this should result in smaller errors on those results. The charm rosult woukd also benchit, aince the vlowed fluctuations in the charm ample backgrounds woukd be smallex.

The only way to effectively decouple the charm result from those for uds and $b$ is

\footnotetext{
This has been aptly demowatrated, ef. by the ALEPH Colfaboration in their moneurement of $\Gamma\left(Z^{*}-\boldsymbol{B}^{3}\right) / \Gamma^{(}\left(Z^{*} \rightarrow\right.$ hedrons $)[\mathbf{7 0}]$.
} 
to obtein a charm ample of high purity. The entabliabed method of reconatructiug $D$

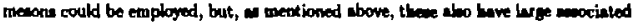
syatematic erron. The ideal solution would be If a mose eficient way of tegaing charm

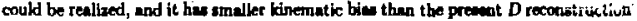
andyaes. Work th in progrew on auch a techsique, but it bas not maturad ax this the. The future of tests of flavor independence could well be in the bolation of high-purtty

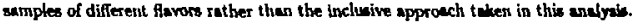

\subsection{Conclusions}

We have presented a test of the flaver independence of the strong interaction by isolating event samples enriched in quarkn of specific asoms (uds, $c$, and $b$ ) and meanuring the atrong coupling in exch eample uning a jet rates alynis. This in the first test of fisvor independence to uee a prectejon microvertex detector to select event sampies of all quark flavors besed on the lifetime content of charged tracka. The etrons coupling for each quark Havor was unfolded from tbe composition of quark Bavors in exch cumple, renulting in

$$
\begin{aligned}
& \frac{\alpha_{y}^{2}}{a_{z}^{2 t}}=0.987 \pm 0.02 \pi(s t a t) \pm 0.022 \text { (syst) } \pm 0.022 \text { (theory) } \\
& \frac{a_{t}^{c}}{c_{t}^{p t}}=1.012 \pm 0.104 \text { (stat) } \pm 0.102 \text { (syst) } \pm 0.096 \text { (theory) } \\
& \frac{a_{1}^{*}}{a_{0}^{*}}=1.026 \pm 0.041 \text { (stat) } \pm 0.041 \text { (syst) } \pm 0.030 \text { (theory). }
\end{aligned}
$$

The effects of quark manes on the jet rates and the full $O\left(\alpha_{0}^{2}\right)$ calculations for the jet rates have been employed in this messurement. The dominant experimental syst tematic errors arise from the uncertalnties in the physics of heavy quark decay. The In'geat theoretical uncertainties are due to the variation in the resulis obtained with the six different fot algorithms used in the andysis, which are presurnably caund by uncalculated higher-order terms in the predictions for jet production rates. These results are the moet precies test of the flavor independence of the strong interaction for light quark ovents; the other mearurements compare favorably with similar results obtained at LEP. 


\section{Part II}

A Measurement of the Parity
Violation in the $Z^{0} c c$ Coupling 


\section{Chapter 13}

\section{Analysis Method}

This chapter contains a description of the reconstruction and selection tachniques uned to isolate hadronic events contuiring charm mesons" These techniques are band upon the reconstruction of $D$ meoons, which are identified by the corbined inveient ane of their decay products. At this point, we inkroduce the symbol $A C B G$, which will be ueed throughout this thesis to denote Random Combinatoric Back Ground avents or other quantities, such ws asymmetries, reluted to or derived from these events'.

Before wo begin the discusaion of the analyais techniques, we meation weveral polnts that are common to all of the cherm selection analyses to swold repeating them later:

- We are allowed to be less restrictive in the quality of tracks required in thi andysis than in the inclusive tagging analysis. The invariant mases obtuined when a mismeasured track is combined with other tracks is cosentially random and thus does not contribute preferentially to background under any of our signal mass peaks. So, we use in this analyais all charged tracks defined by the criteria listed in Chapter $6^{t}$ namely, thoos tracks which have

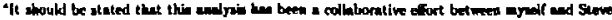

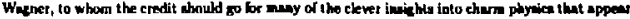
hete.

'This wes nugreated by ky wriets and hande as one why to stave of carpal tumel aydrome until afwe this theis is typed.

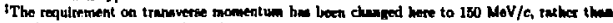
the $200 \mathrm{MeV} / \mathrm{c}$ wed in the QCD andrin.
} 
- tramaverse momentum relative to the beam axis $p_{\perp}>150 \mathrm{MeV} / \mathrm{c}$

$-|\cos \theta|<0.8$

- distance of closest approach in the $x y$ plane $\left(d 0 c a_{x y}\right)<5 \mathrm{~cm}$

- distance of closest approach in the $r z$ plane $\left(\right.$ doca $\left._{r z}\right)<10 \mathrm{~cm}$

with the one alditional constraint that they be well-1neasured enough to have at least one VXD hit.

- The set of tracks combitued to form the charm meson candidates is taken from one thrust hemlsphere at a time. This is done to reduce RCBG from combinations of tracks fron opposite sides of the event that certainly do not come from the same cherm nieson decay. It also speeds up the analysis.

- As will be cliscussed extensively in Section 14.2.1, the mixing-corrected anymmetry in events where a $D^{\text {ot }}$ or $D^{+}$is produced it $b$ decays b almost identical to the standard model expectation for $A_{c}$. Since we wish to measure only $A_{c}$, we hove to amume $A_{4}$ is its standard model valuc, which, Jor = large contamiaation of $b$ cvents in our signal, could result in large systematic errors due to the possible variation of $A_{b}$. So, to minimize the dependence of our results on the exsct value of $A_{4}$, we witl attempt to specifically renowe those $D^{\text {s+ }}$ and $D^{+}$ mesuls which result trom thi. atcluys of $b$ hadrons.

\subsection{The Selection of $D^{\circ}$ Candidates}

As mentioned in Chapter 2, the sample of charm events containing $D^{0}$ decnys is selected using two independent procedures, a kinematic reconotruction analyais and a decay length analysts. The two selected samples are then combined to fon : the final sample used for the asymmetry measurement. Also discused in the opening chapter was the physice of the decay $D^{0} \rightarrow K^{-} \pi^{+} \pi^{0}$, specifically the penking of the $K^{-} \pi^{+}$ mass due to the angular momentum constraints of the decay. Since the reconstructed $K^{-} \pi^{+}$mas in boeth the $D^{0} \rightarrow K^{-} \pi^{+}$and $D^{0} \rightarrow K^{-} \pi^{+} \pi^{0}$ decays has a relatively 
narrow peak, the two selection enalyses for these decay modes are identical, except for the central value of the man region allowed for the $D^{0}$ casdidate in oach cees. The two selection techniques are presented below.

\subsubsection{The Kinematic Technique}

This is the "standiard" $D^{*+} \rightarrow \pi_{*}^{+} D^{0}$ reconstruction technique an mentioned in Chepter 2. It relies solely on the kinematic properties of $D^{*+}$ and $D^{n}$ derays for beckeround rejection.

To begin, all pains of oppositely charged tracks are combined to form a $D^{0}$ candidate by asigning the $K^{-}$insas to one of the particles and the $x^{+}$man to the otber.

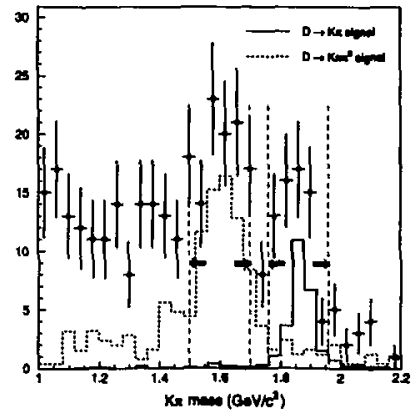

Fisure 33.1: The invariant mase of $K \pi$ peirs after the cuts described in the text. The MC shapes from true $D^{0} \rightarrow K \pi$ and $D^{0} \rightarrow K \pi \pi^{0}$ decays are seo ahown for reference. The relative normalizations of alonal and MC are arbitrary. Aleo ahown are the definitions of the signel regions for the two docay modes. 


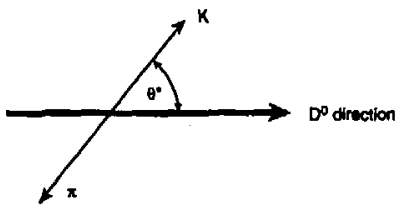

Figure 13.2: Definition of the helicity angle $\theta^{\circ}$, which to the angle between the $K$ direction and the $D^{0}$ flight direction in the $D^{0}$ center-of-nase frame.

We shail refer to the combined inverient mass as $m(K \pi)$. This provides the basic sample of candidate decays on which to base the rost of the selection procedure. The mass peak for rned by a snmple of signal events from the MC is shown in Figure 13.1, along with the data ofter the following culs for the two $D^{0}$ decay modes. The sliape and width of the peaks suggest that the symmetric region of acceptance for candidate events should be $1.765 \mathrm{GeV} / \mathrm{c}^{2}<m(K \pi)<1.965 \mathrm{GoV} / \mathrm{c}^{2}$ for $D^{0} \rightarrow K^{-} \pi^{+}$and 1.500 $\mathrm{GeV} / \mathrm{e}^{3}<m(K \pi)<1.700 \mathrm{GeV} / \mathrm{c}^{3}$ for $D^{0} \rightarrow K^{-} \pi^{+} \pi^{0}$ decays.

Next, we Impose $n$ helicity eut on the deckying $D^{\mathrm{N}}$ system whlch rejects beckgrounds inconsistent with the docay of a spin-zero mcson[201]. This proceeds as follows:

- the lorent $z$ boust of the $D^{n}$ cundidate is calculated based on the total momentum of the $K$ and $\pi$, and the $K \pi$ system is boosted back into the $D^{0}$ rest franje

- the angle $\theta^{*}$, the opening angle between the $K$ momentum and the $D^{0}$ direction in the $D^{0}$ rest frame, is colculated.

This situation is shown pictorially in Figure 13.2. For the isotropic decay of a upinzero particle into two spin-zero particles, one would expect the distritution of $\theta^{*}$ to be fint. Tracks from RCBG that heppen to form a $D^{0}$ candidate with the appropriate mass tend to have their directions parallel to the boost direction in the $D^{d}$ rest frame, as they are unlikely to possess the latge trunsverse momentum of the true $D^{0}$ 


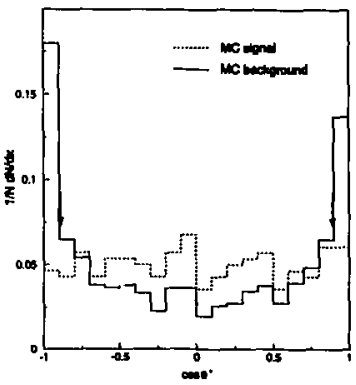

Figure 13.3: Helicity angle distribution for $D^{0} \rightarrow K x$ decaya (dusbed line) and beck. ground (solid line). The arroms show the cut $\left|\cos \theta^{\circ}\right|<0.9$ placed on this variable.

decay products. Thus, some fraction of the background events can be removed with little loss of signal by eliminating events where the decay products lie along the $D^{\circ}$ direction. Wo have chosen to place our cut at $\left|\cos \theta^{\prime}\right|<0.9$. The distribution of $\theta$ for MC signal and background events is shown in Figure 13.3.

The $D^{\mathbf{0}}$ candidate is then combined with $\Perp \pi_{*}^{*}$ candidate track having momentum $p>1 \mathrm{GeV} / c$ and charge opposite to the $K$ - candidate. Requiring a momentum greater than $1 \mathrm{GeV} / \mathrm{C}$ removes some of the contamination due to slow pione from $b$ ducay, as they have a softer mornentum opertrum. A comparison of the $\pi_{0}^{+}$momentum spectra for $D^{\text {ot }}$ decays in $b$ and $c$ cvents is shown in Figure 13.4. The $\pi_{ \pm}^{+} D^{\infty}$ combination formed here will be used to create the $\Delta m\left(\Delta m \leq m\left(D^{n+}\right)-m\left(D^{0}\right)\right)$ distribution which will be used as the "aignal" for the $D^{0}$ andyzis.

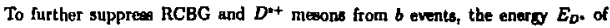
the $D^{\text {At }}$ is calculated, and any $D^{\text {at }}$ candidate with $x_{D^{*}}=2 E_{D^{*}} / E_{C_{M}}<0.4$ i rejectud. This cut takes advantage of the harder charm fragmentation function for 


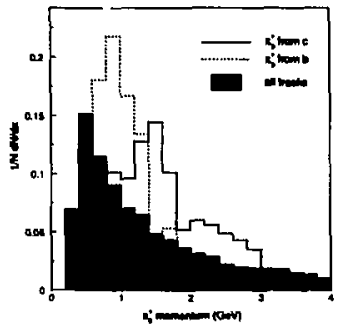

Figure 13,4: Monucrutum of the $x_{0}^{+}$from $D^{++}$decnye in $b$ and $c$ cvents compared with the momentum wpectrum of all tracks.

Table 13.1: The efficiencies for $D^{4}$ decays in $c$ and $b$ events to pan the kinematic selection cuts ussoribed in the text.

\begin{tabular}{|c|cc|cc|}
\hline & \multicolumn{2}{|c|}{$D \rightarrow K \pi$} & \multicolumn{2}{c|}{$D \rightarrow K \pi \pi^{0}$} \\
$c$ cut & $c$ eff & $b$ eff & $c$ eff & $b$ eff \\
\hline$D^{*}$ mas & 0.842 & 0.912 & 0.462 & 0.577 \\
$\theta^{\circ}$ & 0.813 & 0.810 & 0.744 & 0.737 \\
$p_{\pi}>1 \mathrm{GuV} / \mathrm{c}$ & 0.709 & 0.458 & 0.667 & 0.338 \\
$x_{D}>0.4$ & 0.796 & 0.492 & 0.559 & 0.259 \\
$\Delta(m)$ & 0.938 & 0.943 & 0.740 & 0.827 \\
Total & 0.393 & 0.157 & 0.096 & 0.031 \\
\hline
\end{tabular}

$D^{\text {+t }}$ formation. A comparison of the $x_{0}$. distributions for $D^{*+}$ mesons peodued in $b$ and $c$ decryy is shown in Figure 13.5. The efficiencies for each of these cuts for selecting candidate cvents for the two $D^{0}$ decay inodes are given in Thble 13.1.

The $\Delta m$ distribution for this set of selection criteria ts shown in Figure 13.6. for 


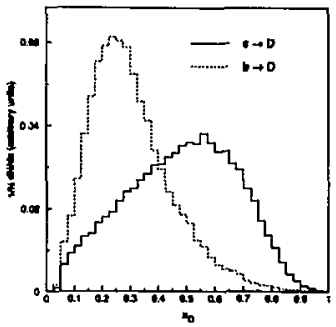

Figure 13.5: Diatributions of $x_{D}$, for $b$ and $c$ decaye.

the two decay modes with the expected aignal shope overlnyed on the datat. We will define the "bignal" region of the $\Delta \mathrm{m}$ distribution to be $\Delta m<0.150 \mathrm{GoV} / \mathrm{c}^{2}$. For this selection, the signal region contalns 65 events for the $D^{0} \rightarrow K^{-} *^{+}$wrode and 100 ovents for the $D^{0} \rightarrow K^{-} \pi^{+}$mode which fall within the $D^{0}$ mes acceptance windows for the two decays. We will also need to incasure properties of the background from the date to reduce our dependence on the MC. Wo define a higher mas "sideband" region of $0.160 \mathrm{GeV} / c^{2}<\Delta m<0.200 \mathrm{GeV} / c^{2}$ for this purpowes?

\subsubsection{The Decay Length Technique}

In the conipletnentary decay length arnlysis, we rely on the fact that $D^{\circ} \dot{s}$ in $Z^{0} \rightarrow c \bar{c}$ events have a long dexay length $(\langle L\rangle \sim 1 \mathrm{~mm})$ and are proniuced at the $2^{\text {toprmary }}$ Vertex (PV) as opposed to being created in a b decay cancude. Since the decay length resolution is $\left\langle\sigma_{l}\right\rangle \sim 200 \mu \mathrm{m}$, clean separation of ovents contuining $D^{\circ}$ decays from

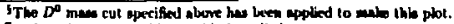

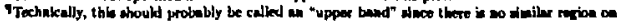

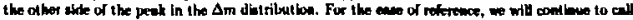
thls a seneric "bidetrand". 

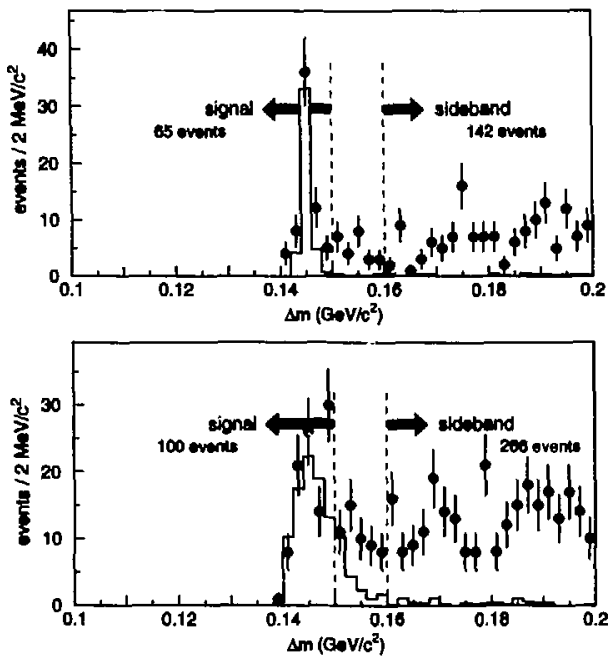

Figure 13.6: The $\Delta n$ distribution for the kinematic sclection andyuis, with the MC pure signal shepe overlayed on the data. The normalization of the MC shepe is arbitray and is meant to indicate the expected signal distribution. The signal and sidebend regions, as well as the number of events they contaln, are aleo shown. 


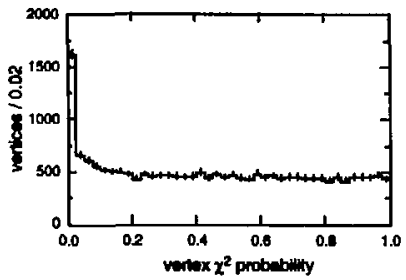

Figure 13.7: The $x^{2}$ vertex probubility for false vertices formed only from tracks originating at the primary vertex. Note the peak near a probability of zero.

RCBG is possible, even at low ID.

The analysin begins with the same pairing of all possible comblantione of oppositely charged tracks to form $D^{0}$ cessidates as in the kinematic analyais, above. The same reatrictions are epplied on the reconst ructed invariant mas.

Next, a constrained vertex fit is performed'; only the track combinations with $\chi^{2}$ vertex probabilitie greater then $1 \%$ are retained $=D^{0}$ candidatea. This correspond to a $1 \%$ low of "true" vertices, which weme like a rather boee cut, except that the false combinatione are peaked sharply at a probability of zero. An example of the vertex probability for combinatoric background is shown in Figure 13.7.

A decay length aignificance cut of $L / \sigma_{L}>2.5$ is then spplied. The values of $a_{L}$ are obtained from the fit for esch of the vertices under conaideration. This cut drastically reduces the number of background vertices that occur close to the PV due to the large apparent track overlap. This is shown pictorially in Fijure 13.8, where the distributions of $L / \sigma_{L}$ are shown for true vertices and those fron combinatoric beckground.

Arother cut using vertex information can be applied to remove a large fraction of

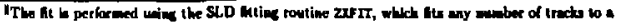

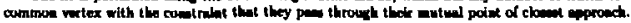




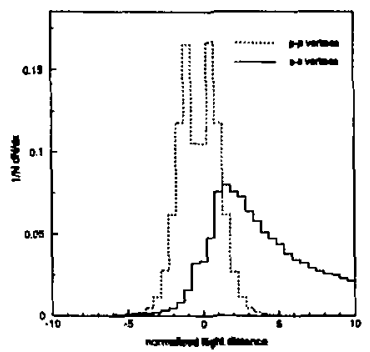

Figure 13.8: The normalized decay length for true secondary vertices and those fake vertices formed from fraginentation tracks due to the confusion near the IP.

the $D^{0}$ incsons produced in $b$ decays. Since the $D^{4 \prime s}$ in charto events are produced at the primary event vertex, their flight path should intersoct the PV. Since D's produced in the b-iecay cascade acquire sigrificant $p_{\perp}$ rehative to the b-hudron flight direction, they may not appear to originate from the PV. The different pomible situntions are shown in cartoon form in Figure 13.9. Given the precision with which the position of the $\mathrm{PV}$ in known within SLD**, this is a power[u] tool for selecting cherm decays. The inpact parnmeters in the $x-y$ plane for the $D^{\prime \prime}$ cantididates from $c$, b, and RCBG events are overlnyed with those measured in the date in Figures 13.10e and 13.10b. Notice that this cut even works for the only-partielly-reconstructed $D^{\mu} \rightarrow K^{-} \pi^{+} \pi^{0}$ decays; the hurd charm fragnentation function ilssures that the charged tracks from the $D^{4}$ decay have sufficient energy to provide an accurate determination of the $D^{n}$ direction. A reistively tight cut on the 2-D inpect parsmeter of the $D^{0}$ momentum vector to the PV of ory $<20 \mathrm{~mm}$ is then applied to further reject RCBG and contamination Gom b events.

\footnotetext{
"See Clepplet 5.
} 
(a)

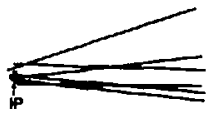

(b)

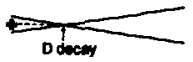

(c)

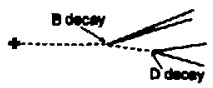

Figure 13.9: Illustration of several of the vertexing situations that can occur when dl tracks are used to form two-prong vertices. Many vertices are formed ckes to the If as in (a) from rundom track overlap. The vertex momentum tenda not to point directly beck at the IP' in this cace. For a $D$ decay in charm events, as in (b), the $D$ is produced at the origin, and the vertex momentum will point back to the IP within measurement errors. The $D s$ produced in a $b$ decay cacede, (c), acquire some tranaverse momentum from the $b$ hedron decay, and thus do not necenarily point back at the IP.

Finnlly, we require only $I_{D^{*}}>0.2$, and make no cuts on $\mathrm{cm} \theta^{+}$or minimum $\pi_{\theta^{+}}$ monientum, since the charm purity of the sample remaining after the prevlows cuts is sufficiently high. Toble 13.2 lists the efficiencies for churm events to pase each of the preceding cuts. Figure 13.11 shows the $\Delta m$ dist ributions for the decay length selection analysis. After applying 11 et $D^{0}$ mass requirentents, 49 events for the $D^{0} \rightarrow K^{-} \pi^{+}$ mode and 73 events for the $D^{0} \rightarrow K^{-} \pi^{+} \pi^{0}$ mode appear in the signal regton.

Comparieon of Figures 13.6 and 13.11 shows that the kinematic and decay length selection techniques yinld $D^{\circ}$ sumple with ewentinlly the sane slgnal to nowe in buth of the recenatructed decay modes. To maximize the available dat., we choose tu combine the results of these two analyses by taking the union of the iwo selected 


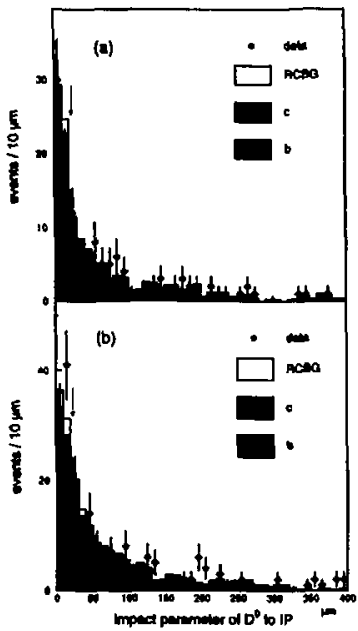

Figure 13.10: The impuct parameters of $D^{0}$ mesors to the IP for the modes (a) $D \rightarrow K \pi$ and (b) $D \rightarrow K \pi \pi^{0}$. Showin are the data points, and the coutributions for vertices from $R C B G, c$, and $b$ decays. The arrowa indicate the cut on this variable, pluced at $20 \mu \mathrm{mm}$. 

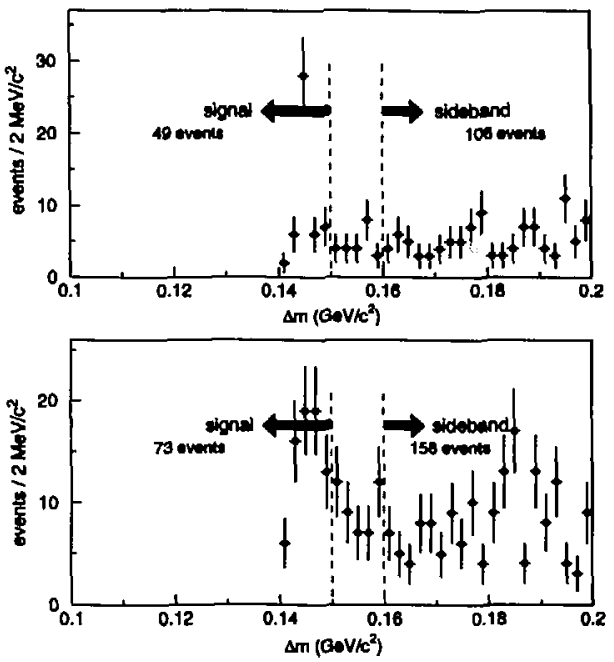

Figure 13.11: The $\Delta m$ distributions for the decay length andysis, data points only. The signal and sideband regions, as well a the number of events they contuin, are also shown. 
Table 13.2: The eficiencies for $D^{0}$ decays in $c$ and $b$ events to pass the decay length election cuts deccribet in the text.

\begin{tabular}{|c|cc|cc|}
\hline & \multicolumn{2}{|c|}{$D \rightarrow K \pi$} & \multicolumn{2}{c|}{$D-K \pi^{0}$} \\
$c$ cut & $c$ eff & $b$ eff & $c$ eff & $b$ eff \\
\hline$D^{0}$ mass & 0.642 & 0.912 & 0.462 & 0.577 \\
vertex $\chi^{2}$ & 0.006 & 0.993 & 0.883 & 0.788 \\
$L / \sigma_{L}$ & 0.524 & 0.731 & 0.402 & 0.643 \\
vertex doca & 0.758 & 0.204 & 0.581 & 0.163 \\
x $_{D}>0.2$ & 0.975 & 0.859 & 0.938 & 0.640 \\
$\Delta(m)$ & 0.989 & 0.973 & 0.798 & 0.650 \\
Total & 0.321 & 0.113 & 0.071 & 0.020 \\
\hline
\end{tabular}

samples and using this as the set of cantiulnte $D^{\text {b }}$ decays for the ssymmetry measurement and background studies. Any errors introduced by not treating potential slight diferences between the two samples is cxjucted to be negligible conpared to the uther measurement errors. The overlap of the two sanples is $(28.4 \pm 5.7) \%$ for the $K \pi$ inode and $(28.2 \pm 4.6) \%$ for the $K \mathrm{xx}^{\circ}$ modeth. The total number of events that apposer in the signal region for the combincd samples are given in Table 13.4.

\subsection{The Selection of $D^{+}$Candidates}

In order to salext a clean enough sample of events contsining $D^{+}$mesons, the selection criterin conprise several of the leatures of the previous two methods.

Cendidaten for $\mathrm{D}^{+} \rightarrow \mathrm{K}^{-} \mathrm{\pi}^{+} \pi^{+}$decnys are formed by combining two tracks of the same sign with one track of the cpposite sign, where all three tracks are required to have $p>1 \mathrm{GeV} / c$. The minimunt momentum requirement serves to lnsure that the track parameters are well-speasured, and results in little loss of efficiency for $D^{+} 8$

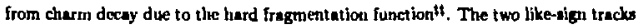

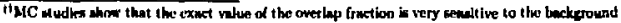
comenamiantion in the sumple. This result will be und in cur systematic atudics, which appoer in Chalker 10.

l'We will andy a cut on fot later, anyway.
} 


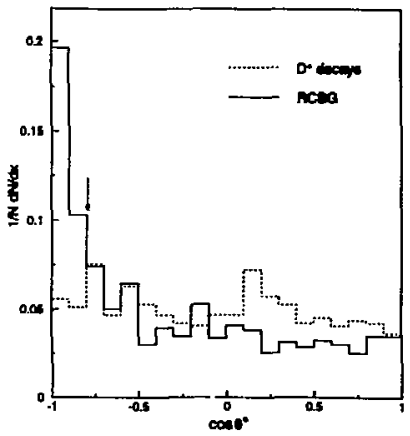

Figure 13.12: The cos $\theta^{*}$ diatribution for $D^{+}$decaye (dashed line) and background (solid line). The arrow shows the cut $\cos \theta^{\circ}>\mathbf{- 0 . 8}$ placed on this variabie.

are asigned $\pi^{+}$menes and the oppowite sign truck is given the $K^{-}$man. A series of cuts is then applied to reject RVSG and $D^{+}$decays from $b$ events.

Fint, we require cos $\theta^{*}>-0.8$, ws the helicity angle cut aleo works on $D^{+}$events. The distribution of $\cos \theta^{*}$ in $D^{+}$events is shown in Fizure 13.12. To roject $D^{\text {t+ }}$ dectys, the differences between $m\left(K^{-} x^{+} \pi^{+}\right)$and $m(K \pi)$ are formed for each of tho two pions and are required to be greater than $0.160 \mathrm{GeV} / \mathrm{c}^{2}$, as this is where the mass difference $\Delta m$ must peak for combinations of a slow pion with $D^{+} \rightarrow K^{-} \pi^{+}$ and other purtially reconstructed $D^{0}$ decay modes. To remove RCBG, wequire $L / \sigma_{L}>3.0$ for the $D^{+}$decay length, which has the mane effect as the cut applied in the $D^{0}$ weiection criteria, above. The cut is 3.00 in this cme because the buckgrounds in this decay mode are more severe; the longer lifetine of the $D^{+}$alows us to tiphten this cut without mush lone of signel. 


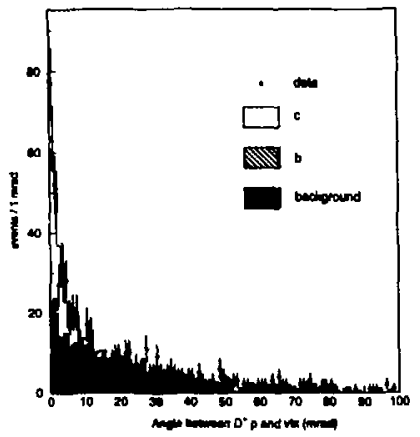

Figure 13.13: The angle butween the $D^{+}$momentum and its flight direction. The contribusiuns from $e$ decays, and the combinution of ACBG and $b$ decays is shown with the data points.

Then, "collinearity" cut is apphied, renuiring the vestex mornentum direction to be collinear with its flight direction. This is essentially the same restriction as the impact parameter cut used in the $D^{0}$ decay length analysis deacribed above, and is made to reject $D^{+}$s from $b$ events ard other RCBG vertices. the angle between the $D^{+}$mornentum vector and the vertex flight direction is required to be less than 5 ared in the $x-y$ plane, and less than 20 mraci in the $r-2$ planc. In contrast to the fixed value of the innpact parameter cut, the collinearity cut corresponds to an impact paraneter cut whose allowed value increases with the decsy length; this type of cut was found to yield better overall signal-to-noise than a standard inifact parameter cut in this particular case. The value of $5 \mathrm{mr}$, though, is more restrictive than the ample impact perameter requirement of $20 \mu \mathrm{m}$, as this requires an impact parancter 


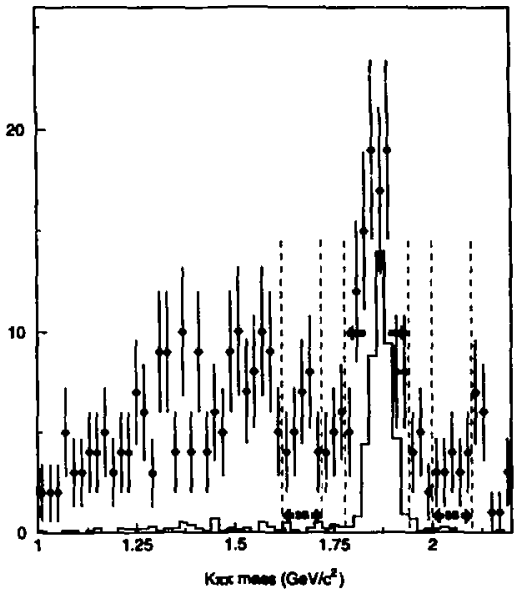

Figure 13.14: The distribution of $K \pi \times$ mames after cuts, with a pure $D^{+} \rightarrow K \pi \pi$ signal shepe overlayed on the data points. The MC shepe has an arbitrary normalization and is slown to provide an indication of the location and shape of the $D^{*}$ signal peak. Also shown are the signal and sidebend regions.

of the vertex momentum relative to the IP of $3 \mu \mathrm{m}$ at a decay length of $1 \mathrm{~mm}$. Mout of the reconstructed $D^{+}$meous in charm events fall within thic cut, however. Plots of the collintarity angle in the $x-y$ and $r-z$ planes for MC sienal events sre shown in Figure 13.13. 
Thble 13.3: The efficienciea for eech of the cuts in the $D^{+}$anajysis for $c$ and $b$ events.

\begin{tabular}{|c|cc|}
\hline & \multicolumn{2}{|c|}{$D^{+} \rightarrow \overline{K \pi \pi}$} \\
$c$ eff & $b$ eff \\
\hline$p>1 \mathrm{GeV} / c$ & 0.655 & 0.494 \\
$\theta^{*}$ & 0.850 & 0.850 \\
$L / \sigma_{L}$ & 0.828 & 0.976 \\
$r y$ angle & 0.452 & 0.832 \\
$r z$ anglr & 0.369 & 0.551 \\
$\Delta(m)>0.16$ & 0.892 & 0.968 \\
$x v>0.4$ & 0.436 & 0.193 \\
$D^{+}$mass & 0.869 & 0.800 \\
Totul & 0.226 & 0.028 \\
\hline
\end{tabular}

Finally, $x_{b^{*}}$ is required to be $>0.4$; even with the alowe cuts, this hard $x$ cut is necesary to achieve a bigh-purity sample of $D^{+}$events. Table 13.3 gives the efficiency of each of the cuts. It is unclear at this time why the efficiencles for retainizig $D^{+}$ decays from $c$ and $b$ events are equal. This may just be a computational error.

The mass peak for pure signal eveuts from MC is shown in Figure 13.14 slong with the events froin the dat a that pas the alowe cuts. After all sclection criterie, $D^{+} \rightarrow K^{-} \pi^{+} \pi^{+}$candidates fall in the range $1.800 \mathrm{GeV} / c^{2}<m\left(K^{-} \pi^{+} \pi^{+}\right)<1.940$ $\mathrm{GeV} / c^{2}$, while the sideband regions are defincol as $1.640 \mathrm{GeV}<m\left(K^{-} \pi^{+} \pi^{+}\right)<1.740$ $\mathrm{GeV} / c^{2}$ and $2.000 \mathrm{GeV} / \mathrm{c}^{2}<m\left(\mathrm{~K}^{-} \pi^{+} \pi^{+}\right)<2.100 \mathrm{GeV} / c^{2}$. The total number of events that appear in the signal region is given in Table 13.4 .

\subsection{Determination of the Buckground and Signal Fractions}

The relative fractions of signal and RCBG romuining after all andyais cutw wate entmated using the $\mathrm{MCC}$ to provide the correct shapsi, then normalizing to the obeorved date smple. The statisties of a study using wrong-sign combinations to 
the background are poor; we will we the wrong-rign diatribution (among other techniques) to estimate the oyztematic error on this procedure.

To obtain the "esymmetry-carrying" charmed hadron nignel thape in both the aifnal and sidebund regions, ali MC everts with three trecks frotn a "correctly-siged" charm decay are taken as signal. For the $D^{0}$ andyrin, thls is a consibination, then, of properly reconstructed $D^{*+}$ decays, partially reconotructed $D^{++}$decays where the $\pi_{\text {: }}^{+}$ (and hence the sign of the charm quark) is properly identified. and a amall fraction of correctly reconstructed threeprong $D^{+}$decays. If the $\pi_{0}^{+}$is mividencified in partially reconstructed $D^{\text {+t }}$ Jeckys where the $D^{0}$ decays into two charged tracke, thete is asentially a $50 \%$ probability that the sign is chosen cortectly from the otbe- two decay products, so "hat the "incorrectly-sigued" partial $D^{\text {s+ }}$ decays contribute no asymmetry. The contributions from four-prong $D^{0}$ decays are small, since the proba-

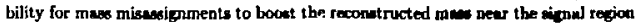
is small. The five-prong branching fraction fur charged $D$ dacays are sipmificantly sualer, so they contribute even leas to the signal fraction. We costimate the reletive fractions of the different contributions in the signal ample to be: $D^{*+}$ :Partial $D^{*+}: D^{+}: 95 \%: 4 \%: 1 \%$. Virtually all of the signel in the sideband region comes from charm events other than the chween modes, however. For the $D^{+}$enalyzin, all charm decays where the three tracks are carrectly awociated with a decaying charm meson are counted as signal, whether they originate from $D^{*+}, D^{+}$, or from other charmed hadrons, including $D_{;}^{+}$. The three tracks from modrs other than the $D^{+}$decayn muat properly determine the sign of the charm quark. Agatu, contributions from five-prong charged $D$ decays are expected to be amall dive to the sulall branching fractions for these modes. The probability for melerting three trecke from the decay $D^{*+} \rightarrow \pi_{0}^{+} D^{9}$ where the $D^{0}$ decays via $D^{0} \rightarrow K^{-} \pi^{+} \pi^{+} \pi^{-}$and misangning the manes is amall. The contributions of the different signal types are: $D^{+}: D^{*+}: D_{a}^{+*}: 98 \%: 2 \%:<1 \%$. The signal contributions to the videbunds do, however, corse exclusively from these other esymmetry-carrying modes, like $D^{+} \rightarrow K^{*}\left(\nu\right.$ or $D^{+} \rightarrow K^{-} \pi^{+} \pi^{+} \pi^{0}$.

The background shape is then taken from all entries is: the xigrid and sideband mass regions that do not come from a 3-prong charmed hedroet deany

To obtain the fractions of signal and beckground in the event sempte, the relative 

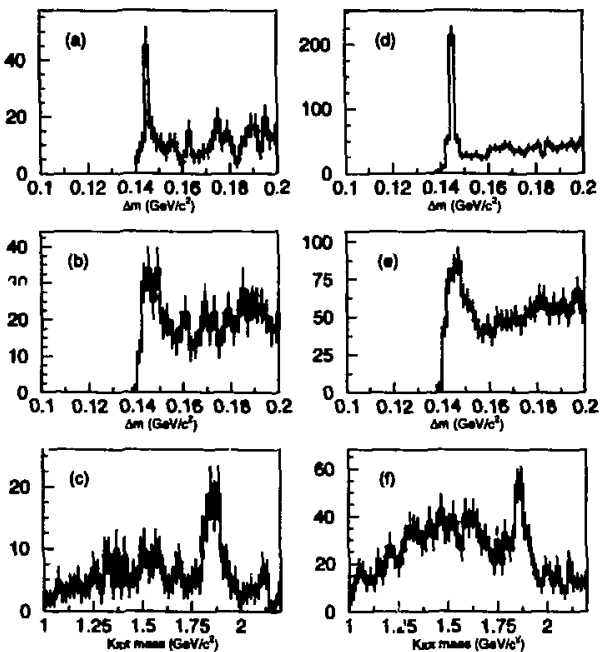

Figure 13.15: The $\Delta m$ and $D^{+}$mas distributions for duta (left column) and ACC (right column) for the three derny modes. Figures (a) and (d) are the distributions for $D^{0} \rightarrow K \pi_{1}$ (b) and (e) are those for $D^{0} \rightarrow K \pi \pi^{0}$, and (c) and (f) are those for $D^{+} \rightarrow K \times \pi$. 
normalizations of the MC signal and MC buckground shepes are selected mect thex the overall diatribution matches that otwerved in the dale in the sipal and inblend regions only. The "dead" regions bet ween the rigal and widebuad will be uod for

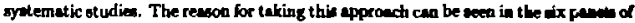
Figure 13.15, where we show the dientribution in $\Delta m$ and $m(K \pi x)$. for dele and MC. It is obvious that the MC predictions for the signal shape does not malch the dela for the $D^{+} \rightarrow K \pi \pi$ mode. The dintribution for the $D^{0} \rightarrow K \pi \pi^{0}$ mode are alop difmen. This is due to soveral incorrect production crom actions and brasching fractioin $\mathbf{l}$ the MC which will be diecued below. Adjuting the reletive normalizations of the signal and background shapes fres un from neating the MC to pintict the cheotule level of the background. The errord on the relative fractions will be included $\boldsymbol{a}$ a uystematic error in Chopter 16.

The rewalts of this beckground calculation procedure are thone in Firow 13.14, 13.17, and 13.18, where the thapes $\alpha$ the $D^{\prime} \Delta \mathrm{m}$ and $D^{+}$man ditsibution and the est insuted beckground and signal shapes are plotted aloag with the experimental dats. We see that good agreetrent between the MC and dats is now obsuined. To maln this agreentent posible, $18 \%$ more signal wee necemery in the $\Sigma^{0} \rightarrow K \pi$ mode, $4 \%$ thore signal was necesary in the $D^{\infty} \rightarrow K \pi \pi^{*}$ mode, and the branching frection for $c \rightarrow D^{+}$bad to be increaced by $35 \%$ in the $D^{+} \rightarrow K$ ma morte. Some explanation kor the variation between MC and date uhould be put forth here. When the prodictions of the MC diesgres with the date, the MC parameters immedietely becomen anpact. We have checked in detail tbe values of production rates and branching frection for charm mesons in tho SLD MC, and orme of our finding serve as explenatione for tha normalization differences. One majur flaw of the MC simulation w that the ratio of the rate for vector meon to that for vectos + peesudacelar meaco production in cheran decays $\left(R_{v} /\left(R_{v}+R_{v}\right)\right)$ is set to $3 / 4$ in the MC inotead of the 0.55 mewured in b decays". This hes the effect of suppresing the rate of $D^{+}$production in cherm decayn, which explains the differences between data and $\mathbf{M C}$ in the $\boldsymbol{K}^{-} \mathrm{x}^{+} \mathrm{x}^{+}$men dialribution. Once a compenuting $35 \%$ nore $c \rightarrow D^{+}$decays bave beon added, the aromant between MC prediction and the oberved date dintribution is rood. The bearnece

\footnotetext{
- See Chapter 16 for reference.
} 
value of $R_{v} /\left(R_{0}+R_{p}\right)$ should impact tive $D^{0}$ distributions in the opposite direction, bowever, a it would imply that more $D^{*+}$ 's are being produced in the MC then in nature. We need more sigmal in the MC to match tlee oberved distributions, though, which does not match our expectations. We have checked the $D^{*+} \rightarrow D^{0}$ branching fraction, and the MC value of $67.9 \%$ matches well the PDC value of (68.1 $\$ 1.3) \%[194]$. The $D^{\mathbf{W}} \rightarrow \mathrm{K}^{-} \pi^{+}$branching fraction is the MC in too $\mathrm{mm}$ l, $3.57 \%$ compared with the PDG $(4.01 \pm 0.14) \%$, which would explain the $18 \%$ difference in the aignal fractions between datu and MC if we believed that $R_{0} /\left(R_{v}+R_{n}\right)$ is correct lo the MC. Wh should be able to distinguish whether the relative fractions of $b$ and $c d$ 'in the MC matches what ne observe in the data by comparing the siguel to $b$ ratioe in the decey lengtin us. the kinematic andysis while cuts are varied, sprti-iay the $x_{D}$ and pointivg cuts, as this should changu the relative fraztions of $b$ and $c$ events differently in the two samples depending on the ratio of $b$ to $c$ content of the $D^{0}$ sample. These atudiwe are still under consideration, and no clear explanstion for the difference between MC and date for the $D^{0}$ modes exists. The fraction of double-tagged events does kend creelence to the results of our backgrnund calculations, however; chapter 16.

Thrac plots also show that the mass rejolution of the tracking system is more or leos correctly sinulated in the MC'. The fractions of events in the signel regions that come from tharm $\left(f_{-\infty}\right), b$ events $\left(f_{b}-D\right)$, aud RCBG $\left(f_{\text {manc }}\right)$ are shown in Table 13.4 for the signal region. The asne quantitios for the sideband regions are given in Table 13.5.

\subsection{The Raw Asymmetry}

At this point, can displiny the keft-right forward-backward symmetry present in the relected dare. Figure 13.19 shows the distributions of the outgoing fermion directions $\mathrm{a}$ deternitied by the sign of tise $D$ newon for the two different beam belicity

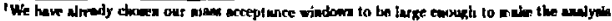

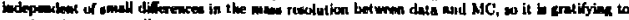
ner thes thay weter wedl.
} 


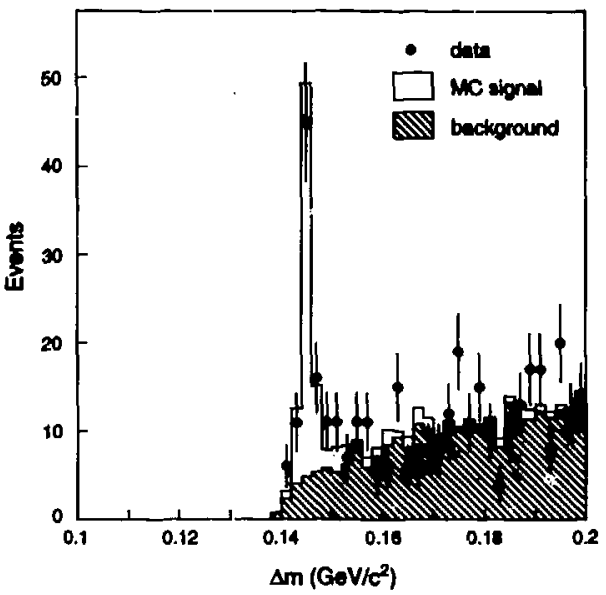

Figure 13.16: The $\Delta m$ distribution for data, MC signal and RCBC for $D^{0} \rightarrow K x$. The relative normalizations of the MIC signel and RCBG shapes ha been obtained by matching the number of dats events in the aignd and aidetind regions. 


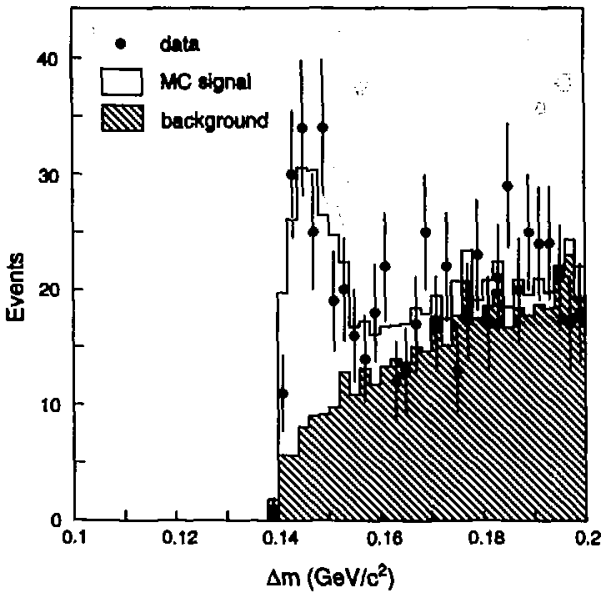

Figure 13.17: The $\Delta m$ distribution for data, MC signal and RCBG for $D^{0} \rightarrow K \pi \pi^{0}$. The relative normalizations of the MC signal and RCBG sliapes has been obtained by motching the number of data events in the signal and sideband rcgions. 


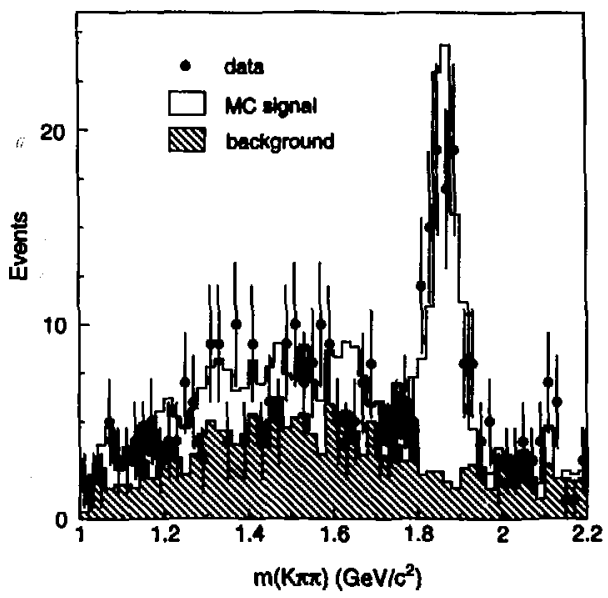

Figure 13.18: The $\triangle m$ diatribution for data, MC signal and RCBG for $D^{+} \rightarrow K \pi x$. The relative normalizations of the MC signal and RCBG shapes hes been obtained by matching the nunber of data events in the aignal and sidebend regions, with the addition of $35 \%$ moro $c \rightarrow D^{+}$evento. (See text.) 
Table 13.4: Total number of signel events and the estimated fractions of c and $b$ signal and RCBG for the three charm decay modes

\begin{tabular}{|c|c|ccc|}
\hline Decay Mode & \# of Signal Events & $f_{c \rightarrow D}$ & $f_{L \rightarrow D}$ & $f_{\text {nran }}$ \\
\hline$D^{*+} \rightarrow \pi_{+}^{+}\left(K^{-} \pi^{+}\right)$ & 88 & 0.52 & 0.22 & 0.26 \\
$D^{++} \rightarrow \pi_{s}^{+}\left(K^{-} \pi^{+} \pi^{0}\right)$ & 131 & 0.50 & 0.22 & 0.28 \\
$D^{+} \rightarrow K^{-} \pi^{+} \pi^{+}$ & 08 & 0.70 & 0.14 & 0.16 \\
\hline
\end{tabular}

Table 13.5: Total pumber of sideband events nnd the estimated fractions of $c$ and $b$ sigual and RCBG in the sidebands for the theer charen decay modes

\begin{tabular}{|c|c|ccc|}
\hline Decay Mode & \# of Signal Eyents & $f_{c \rightarrow 0}$ & $f_{L \rightarrow D}$ & $f_{\text {ncea }}$ \\
\hline$D^{++} \rightarrow \pi_{+}^{+}\left(K^{-} \pi^{+}\right)$ & 230 & 0.017 & 0.036 & 0.917 \\
$D^{++} \rightarrow \pi_{+}^{+}\left(K^{-} \pi^{+} \pi^{0}\right)$ & 398 & 0.573 & 0.044 & 0.883 \\
$D^{+} \rightarrow K^{-} \pi^{+} \pi^{+}$ & 45 & 0.162 & 0.020 & 0.060 \\
\hline
\end{tabular}
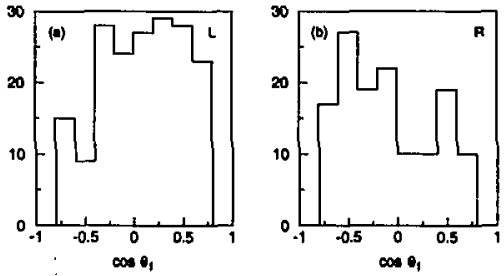

Figure 13.19: The direction of the outgoing $D^{(*)+}$ for $Z^{0}$ evente produced with electron beams of left-handed (a) and right-handed (b) helicitles. The angle shown is the direction of the outgoing $D^{(+)+}$meson contalning the $c$ (ns opposed to the d) quark. 
states. Even with the low number of events, a large formard beckword aymatry cas

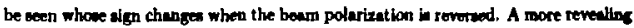
manner of displaying the aymmetry in to revere the plot for the right-handed bean and overlay this on top of that for the left-handed beam. Thin correspowde to forming the first set of sums in the formula for $\lambda_{F E}$ from Eq. 2.72

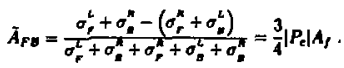

This distribution is sh wn in Figure 13.20. The beckground symmetry shown on this plot is taken frotn the sidebends, and has hed the mymimotry from the slgral continination of the sidebands subtracted. Note the lerge raw aymmetry. Thin shows in a very visual manner the edvantages of meanuring the aymmetry uning a longitudinally polarized beam.

We turn now to a discussion of the maximum likelibood analyis uned to extract $A_{c}$ from the charm sample. 


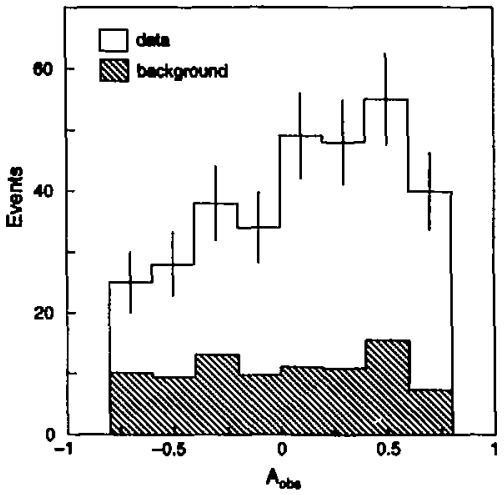

Figure 13.20: The forward backward anymmetry, combining the two polarization states by adding the distribution of $\cos (-\theta)$ for those events produced with a rightbanded electroa beum (see Fig. 13.19) with that of $\cos \theta$ for those events produced with a left-handed electron beatn. The asymmetry shown from the background is from the sidebands with thu appropriate amount of MC signal symmotry subtracted. 


\section{Chapter 14}

\section{The Asymmetry Likelihood}

\section{Function}

\subsection{Formulation of the Likelihood Function}

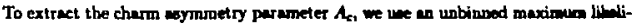
hood fit[202] bewed on the Born-level cron wetion for fermion production in $Z^{0}$-boen decay from Eq. 2.56:

$$
\frac{d \sigma}{d \cos \theta} \times\left(1-A_{\theta} P_{\varepsilon}\right)\left(1+\cos ^{2} \theta\right)+2 A_{f}\left(A_{c}-P_{c}\right) \cos \theta \text {, }
$$

The fitting procedure used here in aimilar to that uned by the OPAL collaboration in Ref. [203]. In this nection, we give en overview of the likelihood fittins metbod. A detailed discussion of the fit perameters will be prosented below.

The likelihood function should have the general form

$$
\log C=\sum_{i=1}^{n} \log \left(P \cdot \theta\left(y_{i}\right) \cdot\left(1+y_{i}^{2}+A_{f} \cdot y_{j}\right)\right),
$$

where $y=q \cdot \cos \theta, q$ is the sign of the $D^{-+}$or $D^{+}$churge, and $\theta$ in taken bere to be the angle between the $D^{\text {+t }}$ or $D^{+}$mewon momeatum and the electron beam. Here, $n$ is the total number of events. The function $P$ is a aormalization factor, and $\theta$ parametrien. any non-uniform acceptance a a function of $y$. Nose that kny goometric acouptape 
cymmetry chould be indepencient of the $D$ charge, and is thus an even function of $y_{\boldsymbol{i}}$. Terme depeoding on $\theta$ and the overall normalization merely add a conutant term in the above rum and thus cannot weet the aymmetry parameter givet by minimizing $\boldsymbol{c}$. We will talue up the question of nom-uniform acceptence apain in the discussion of the ayatematic errors.

The actual form of the likelibond function used in this aralyeis is

$$
\begin{aligned}
& \log \mathcal{L}=\sum_{i=1}^{n} \log \left\{P_{t}^{j}\left(x_{D i}\right) \cdot\left[\left(1-P_{e} A_{e}\right)\left(1+y_{i}^{2}\right)+2\left(A_{\varepsilon}-P_{e}\right) y_{i} \cdot A_{i}^{D} \cdot\left(1-\Delta_{\mathrm{u} \cup 0}^{b}\left(y_{1}\right)\right)\right]\right. \\
& \left.+P_{r}^{j}\left(x_{D i}\right) \cdot\left(11-P_{r} A_{e}\right)\left(1+y_{i}^{2}\right)+2\left(A_{e}-P_{e}\right) \nu_{i} \cdot A_{c}^{0} \cdot\left(1-\Delta_{Q(D)}^{0}\left(y_{i}\right)\right)\right\} \\
& \left.+P_{n C E R}^{j}\left(x_{D i}\right) \cdot\left[\left(1+y_{i}^{2}\right)+2 A_{\text {MCMG }} y_{i}\right]\right\}
\end{aligned}
$$

where $A_{b}^{D}$ and $A_{c}^{D}$ are the noymmetrics from $D^{*}$ and $D^{+}$decnys in tagged $b_{\bar{b}}$ and $c \bar{c}$ events, respectively. The index $j$ is used to indicate that each of the three charm decay modes are considered separately, so that the total likelihood in ectually the oum of three parts:

$$
\log C=\log C_{D^{0} \rightarrow K x}+\log \mathcal{L}_{D^{0} \rightarrow K+z^{0}}+\log C_{D^{+} \rightarrow K a x}
$$

The quantity $\Delta_{\text {ofo }}^{f}(y)$ is the $O\left(\alpha_{*}\right)$ QCD radintive correction to the nsymmetry,

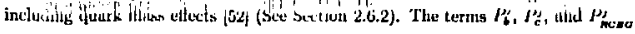
represent the probubilities that an ewent from one of the $j \quad 1,2,3$ decay modes is either signal from $b \bar{b}$ or $c \bar{t}$ events or random combinatoric buskground, depending on the $D$ candidate chergy. An event is uniquely assigned to a decay mode, and to thus only counted once; les than $0.5 \%$ or events are double-tagged, so this las a negligible effect on the andynis.

In the next scaion, the detalhed uxsumptions involved in the mpecification of the functions $P^{\prime}$, and thu uther values which must be inpus to the likelihood function are discused. 


\subsection{Inputs to the Likelihood Analyais}

\subsubsection{Determination of Anymmetrie}

Since the number of $D$ events avilable for this mewuresnent aro limited, we beve chosen to input the values of the mymmetries $A_{b}^{p}$ and $A_{\text {nesa }}$ to the fit, and the fit only for $A_{c}^{D}$ E $A_{c}$. Another reacon for sopting this approech is the value of $A_{P}$, which, after cotrecting for $B^{0} B^{0}$ mixing, is almont identical to the atandard roodel value for $A_{c}, 0.67$. Since the a dirtributions of the $D_{2}$ from b events from $D_{3}$ from $c$ evente owerlap to a large extent and the difference in $D$ energy is the oaly variable we use to separate $b$ and $c$, this coincidenos of values would kend to a fer wore eror on our valuo of $A_{e}$ then if we fixed $A_{k}^{D}$ to its atanderd model value and coneldered suitable errors about this point. Future versions of this andymen may be sentitive enough to the differences between $b$ and $c$ events to allow $A_{b}^{b}$ to toat in the fit*.

\section{Determination of $A_{b}^{D}$}

In order to arrive at the proper value of $A_{b}^{P}$ to be included in the fit, we muat tale into occount the dilution of the $b$ quark exymmetry due to $b$ mixing axd poesibte efrets

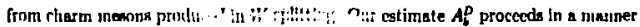
similar to that of Ref [203] and Ref [67], where the corrections to $A_{4}$ to obtain the observed asymmetry $A P$ are parametrized by

$$
A_{b}^{D}=A_{b} \cdot\left(1-2 x_{\text {mir }}\right)\left(1-2 \times w_{F \rightarrow 0}\right) \text {. }
$$

We begin with tho Standard Model prediction of $A_{b}=0.935$ [51], and allow this to vary by \pm 0.105 to aceommodate the meweurements from LEP and SLD [204]. The fraction of $D^{++} \propto D^{+}$, deroted os $D^{(0)+}$ collectively, corning from the spectator part of $B_{d}$ decaya (Figure 14.1a) is taken half way between iso two extrems, which are that either all $D(0)+$ mesons in $B$ decaye come onily from $B_{d}$ decays, $\alpha$ that there in no preference for $D^{(*)+}$ coming from any particular type of $B$ decay $\left(B_{n}, B_{d}, B_{n}, A_{*}\right)$

\footnotetext{
"See the dlarialon in Chapter 17.
} 
(Figure 14.1b). In the first case, the mixing parameters $x_{2}=0.16 \pm 0.025$ neasured at CLEO 205$\}$ should be used to calculate the value of $A_{b}$ after mixing. For the second cave, the average mixing parameter $\bar{\chi}=0.12 \pm 0.01$ mewured at LEP[123] should be used. We choose to tabe the sverage of these two extremes, which rosults in a mixing correction factor $1-2 \chi_{\text {mis }}=0.72 \pm 0.09$. The error on $X_{m i x}$ is obtained by taking the measured $x_{d}$ value, od ling 1 standard deviation, and subtracting $\bar{x}$ :

$$
\delta\left(\chi_{\text {mix }}\right)=\chi_{1}+\sigma\left(\chi_{d}\right)-\bar{\chi} .
$$

The correction for wrong-sign $D^{(*)+}+$ from the $W^{-}$in $b \rightarrow \mathrm{cW}^{-}, W \rightarrow \bar{c}$ (Figure 14.ic) is expected to be small, since the estlmated branching fraction for this splitting to $D$ mesons is estimated to be at most $1 \%[203]$. We conservatively take $\chi_{w \rightarrow c s}=$ $0.025 \pm 0.025$ to encompass a large posesible range for this effect. These two correetions combine to give a value

$$
A_{6}^{D}=0.64 \pm 0.11,
$$

wbere the enor is taken as an experimental systematic error. This valuc is consistent with those obtained by ALEPH[67] and OPAL[203].

(a)

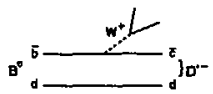

(b)

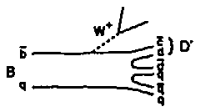

(c) $\mathrm{B}$
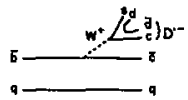

Figure 14.t: Possible sources for $D$ mesons in $B$ decays. Ds can come specifically from $B$ decays (a), nonspecific $b$ hadron decays (b), or from $W \rightarrow$ cs aplitting (c). 


\section{Daternimation of $A_{\text {maso }}$}

With proper subtraction of the fraction of gignal in the sideband repion as given in Table 13.5, we measure an swerage sideband wymretry of $A_{\text {moso }}=0.05 \pm 0.10$. all of the sideband data from the three decay modes give consiatent resulte'. Since the meatured aymmetry in the sideband regions is consintent with aro, we coume $A_{\text {ncac }}=0$ for the central velue and consider pasible deviations from zero a a systertatic error.

\subsubsection{Formulation of the Functions $P(x)$}

The determination of $P_{f}\left(x_{D}\right)$ is besed on the relative frections and the $x_{D}$ diatributions for the three decay modes. The functions $P$ can be fectorived in the following manner:

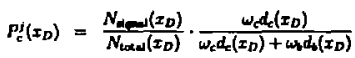

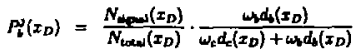

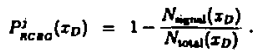

Hete, we have introduced the symbols $\omega_{c}$ and $\omega_{1}$ to represent the fraction of the signd that is due to $D^{(s)+}$ from $c$ and $b$ events, respectively; the values are derived from Table 13.4.

The ratios $N_{\text {demal }} / N_{\text {toted }}$ are calculated by

1. The distributions of $x_{p}$ from the sidebands and from a MC anple containing only $D^{(*)+}$ from $b$ and $c$ events are normalized so that the integral over the distribution yields unity.

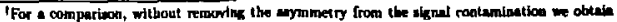
$A_{A C \operatorname{DO}}=0.12 \pm 0.09$.
} 
2. The fractions of the $x_{D}$ dintrilution from the $M C$ signal is subtracted from that of the sidebands, with the relative proportion of signal and backeround given by Table 13.5. The sidelund diat ribution is renormalised back to unity integral.

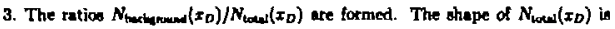
taken from the data. The fractions of background used to form the ratio art taken from Table 13.4. The ratio is caiculated in bins of width 0.05 due to limited statiotics.

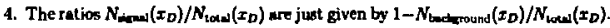

Note that the MC is only used in this procudure to estimate the fraction of signal in the sidebands, and for the $x_{D}$ distribution of the pure signel. Figures 14.2a through c show the contributions to the $x_{p}$ distributions estimated from this procedure overlayed on the data. Note that the poor statistics in the sideband regions results in ratios $N_{\text {danes }}\left(x_{D}\right) / N_{\text {cond }}\left(r_{v}\right)$ that have sigaificent bin-to-bin changes. This will be considered later in the discussion of systematic errors.

The functiuns $d_{C}\left(x_{D}\right)$ and $L_{b}\left(x_{b}\right)$ are parametrizations of the energy distributions of the decaying $D^{(0)+}$ mesons, where the shapes of the two energy distributions are taken from MC. Thus, for a given value of $x_{D}$, the ratio $\omega_{c} d_{c}\left(x_{D}\right) /\left(\omega_{c} d_{c}\left(x_{D}\right)+\right.$ $\omega_{k} d_{b}\left(I_{D}\right)$ gives the probability that a $D^{(*)+}$ candidate is from a primary $c \bar{c}$ or $b \bar{b}$ event. The energy spectra are generated separately for the vector and pseudoscaler D6. A fit to a parametrizing function is then performed for each of the spectra, and the $f \mathrm{~s}$ are normalized to unit area by an overall constant. The charm anmple is fit to the Peterson fragmentatinu functlon(122):

$$
d_{C}\left(x_{D}\right) \propto \frac{1}{x_{D} \cdot\left(1-\frac{1}{x_{D}}-\frac{\epsilon_{D}}{1-x_{D}}\right)^{2}} .
$$

The fitting function for $D^{(\cdot)+}$ mesons from $b$ decays was introduced by the OPAL colleboration[206]:

$$
d_{b}\left(x_{D}\right)=A \cdot \exp \left(\frac{-\left(x_{C}-B\right)^{2}}{C}\right) \cdot\left(1+\frac{D}{x_{D}}+E \cdot x_{D}^{2}+F \cdot x_{D}^{2}\right) .
$$



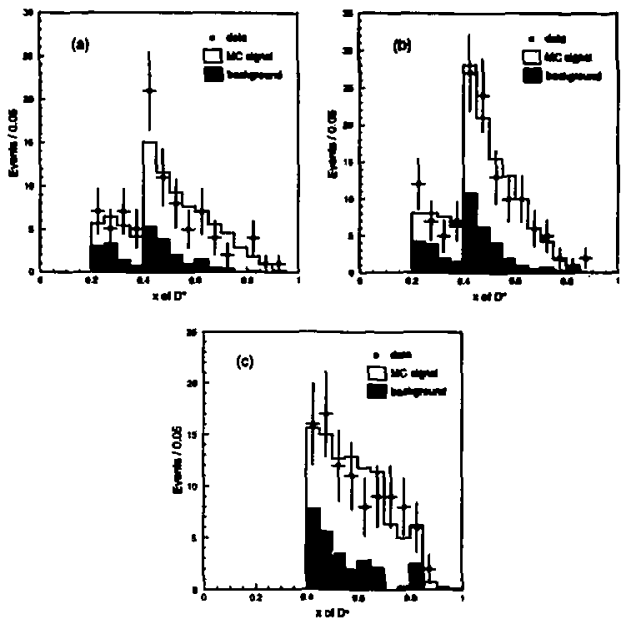

Figure 14,2: The $x_{D}$ distributions for data (points), beckground (filled histogrem), and $\mathrm{MC}$ ajgnal (open histogram), for the decay modes (a) $D^{0} \rightarrow K \pi$, (b) $D^{0} \rightarrow K \pi \pi^{0}$, and (c) $D^{+} \rightarrow K \pi \pi$. The structure at low $I_{D}$ results from the bower $I_{D}$ cut used in the decry length selection of $D^{n}$ decay modes. 


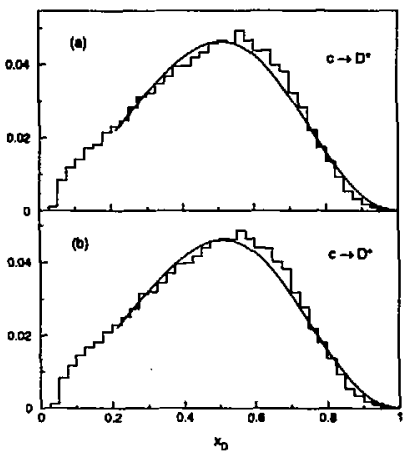

Figure 14.3: The AIC distributions of $x_{c \rightarrow b}$ and the fitted Peterson parametrizstions for (a) $c \rightarrow D^{*}$ and (b) $c \rightarrow D^{+}$. Note that the fragmentation function for $c \rightarrow D^{+}$ is slightly softer, as one might expect. Ser Equation 14.9 for the functionsl form.

Variations of the shape of tlicse functions will be considered in the discussion of the systematic errors. Figures 14.3 and 14.4 show the fitted $d$ functions for charm and $b$ samples, respectively, for $D^{*+}$ and $D^{+}$fragnentation.

Note that, given the definitions above, the total probability for each candidate sujns to unity, as it should.

\subsubsection{Other Inputs}

The value of the polarization $b$ takcn to be $63.0 \pm 1.1 \%$, as discussed in Chapter 4.

We have takeu $A_{f}$ to be equal to the value from the Particle Duts Group[194], $A_{e}=0.1617 \pm 0.012$, where the crror is chosen to cover botls the SLD and LEP central 


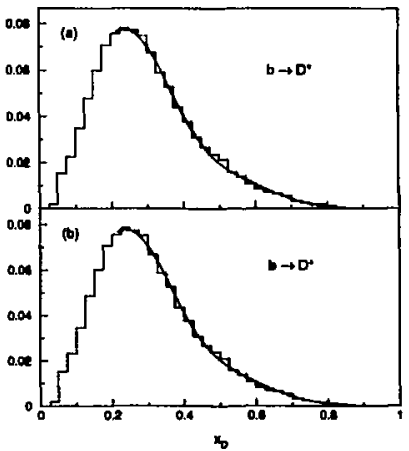

Figure 14.4: The MC distributions of $x_{b \rightarrow p}$ and the fitted parametrization for (a) $b \rightarrow D^{*}$ and (b) $b \rightarrow D^{+}$. See Equation 14 . 10 for the functional form.

values.

\subsection{Results of the Fit}

Performing the maxinum likelihood fit to the data sample, we obtain the value

$$
A_{c}=0.71 \pm 0.20 \text { (stat) }
$$

The minimization of the nezative log-likelihood is shown in Figure 14.5. 


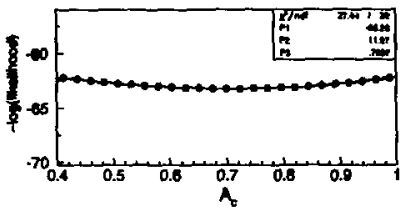

Figure 14.5: The minimization of the libelitood function. The parabolic fit parameters are given by $y=P 1+P_{2} *(x-P 3)^{2}$.

\subsection{Checks of Fit Value}

To cross-check the value of $A_{c}$ abtuined from the fit, we fontued the bin-by-bin forward backward asymmetry using the duta as presented in Figure 13.20 from the previous chopter. We tan fit this asymmetry to the $\cos \theta$-dependent form of $\bar{A}_{F B}$, which wes given in Eq. 2.72:

$$
\bar{A}_{F B}^{c}(z)=\frac{\left(\sigma_{L}^{c}(z)-\sigma_{s}^{c}(z)\right)-\left(\sigma_{L}^{c}(z)+\sigma_{n}^{c}(z)\right)}{\sigma_{2}^{c}(z)+\sigma_{n}^{c}(z)+\sigma_{L}^{c}(z)+\sigma_{n}^{c}(z)}=\left|P_{\mathrm{e}}\right| A_{\varepsilon}-\frac{2 z}{1+z^{z}},
$$

where $z=\cos \theta$, and $L(R)$ denotes that the $Z^{0}$ was produced with an electron beam of left-handed (righthanded) helicity. We performed this fit with four and cight bins in $z$ to check the effects of binning statistics. The resuits are shown in Figures 14.6m and $b$. The result br the fits when the appropriata frection of anymmetry from $b$ events is subtracted are:

$$
\begin{array}{ll}
A_{c}=0.68 \pm 0.23 & (\text { Alin }) \\
A_{c}=0.71 \pm 0.24 & \text { (8bin) }
\end{array}
$$

which are in perfectly good agreement with the likelihood fit value.

We now turn briefly to a discussion of the radiative corrections that mist be applied to this value to obrain the pole value of the asymmetry, $A_{c}^{\mathrm{g}}$ and then to a 
treatment of the posible syntematic errors that efect this mensurement.
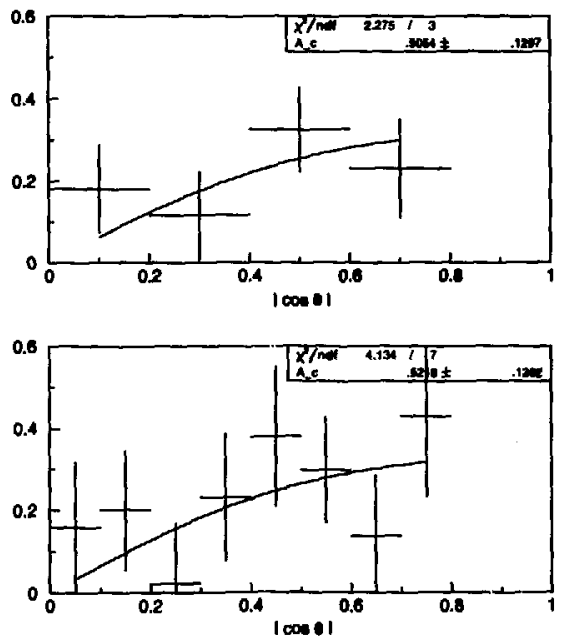

Figure 14.6: Rewults of fitting for $A_{c}$ with a binned aymmetry. The functional form is given by $\ddot{A}_{r b}=|z| /\left(1+z^{2}\right) \cdot 3 / 4 P_{c} A_{c}$, where $z=\cos 0$, and we have taken $A_{c}$ a the free parameter. The fit reult must be corrected for background fraction and b contarnination to obtain the values for $A_{e}$ sives in the text. 


\section{Chapter 15}

\section{Radiative Corrections:}

Obtaining $A_{c}^{0}$

Electroweak und strong interaction radistive corrections need to be applied to the precedisg chapter's result to obtain the value of the Icrinion asymmetry purameter at the $Z^{0}$ pole, $A_{c}^{0}$. The nature of these corrections was discursed in Chapter 2 , so we will only remind the resder briefly hore what these corrections entail.

\subsection{Electroweak Corrections}

Electroweak corrections are included from initial and final statc radiation, vertex corrections, $\gamma$ exchange, and $\gamma-Z$ interfetence". These were calculated using the progran 2FITTER[51], with the inputs $m_{\ell_{0}}=175 \mathrm{GeV} / c^{2}$ and $m_{H, g y:}=300 \mathrm{GeV} / c^{2}$. The Bum of these corrections is an upward conection of $0.8 \%$ to the uncorrected rcsult. The small size of this correction illustrates the robutness of this measurenent to other electroweak uncertainties, as tnost of the large clectrowcak corrections otcur in the $Z^{0}$ and $\gamma$ self-energy terms, which are absorbed into $\sin ^{2} \theta_{W}^{\prime \prime \prime}$ and thus do not affect the measurements of the final state couplings as mucli as they do other nessurements; for the unpolarized $A_{F B}^{f}$, measurad at LEP, this radiative correction is $\sim 10 \%$ of the unconected result $[5 t, 207,208]$. Since the size of the correction is so

- See Section 2.0.1 for a de1ailed dixcumion. 
Table 15.1: The Fraction of QQQQQ or q9QQ events, collectively denoted a $4 Q$ eveats, which pass our event selection cuts, talmalated for $4 Q$ ovents from each of the three types of primary nuarks.

\begin{tabular}{|cccc|}
\hline Enctgy fraction & uds $4 Q$ everits & $c 4 Q$ events & $b 4 Q$ events \\
\hline$x_{Q}>0.2$ & 0.791 & 0.778 & 0.789 \\
$x_{Q}>0.4$ & 0.402 & 0.397 & 0.374 \\
\hline
\end{tabular}

small, we will ignore powsible variations of the correction due to uncertsintied in the Higgs and top quark masses, as these are expected to be negligible.

\subsection{QCD Corrections}

The final state QCD corrections for masive quarks have been calculated to recond order[54]. The second order correction depends in detail on the wcceptance of andyoin cuts for four-jet events wherein a rediated glwon emerges a a pair of heavy quarb, and is given by

$$
A_{c}^{\text {meas }}\left(\mathcal{O}\left(\mathrm{a}_{a}^{2}\right)\right)=A_{c}^{0}\left(1+\left(\frac{a_{a}}{\pi}\right)^{2}\left[-(4.4 \pm 0.4)-(26 \pm 6) f_{o_{n}} l\right),\right.
$$

where $f_{D_{B}}$ is the fraction of these events which pasg our analysis cuts. The other uncertaintics are due to the unknown values of the quark mases. We estimated $f_{D}$. using the $\mathrm{MIC}$ to st udy the energy fractions $x_{Q}$ carried by the henvy quarks in these ewetts. The results are shown in Table 15.1. In our data ample, there are 55 events with $x_{D}<0.4$, so the proper value of $f_{0}$ to take is

$$
\left\langle o_{B}\right\rangle=\frac{55}{317} \cdot(0.8)+\frac{262}{317} \cdot(0.4)=0.47,
$$

where we have taken the average value of 0.8 and 0.4 from the table. We waign an croor of \pm 0.25 on the quantity $f_{D_{b}}$ to cover our relative ignorance of the rate of $4 Q$ events in the MC. When combined with a value $[194]^{\dagger}$ of $a_{f}\left(M_{f}^{J}\right)=0.119 \pm 0.02$ and

\footnotetext{
THe hnw allomed a kenemaje etrot on 0.
} 
the other theoretical uncertsintiea in the above equation, this value of $f_{D}$, give a whe of $-(2.3 \pm 1.0) \%$ for the correction, mear ing that $A_{c}^{0}=A_{c}^{m e n} /(1-0.023)$.

Including all radiative correction, the find result becomes

$$
A_{t}^{0}=0.73 \pm 0.22 \text { (stat) }
$$




\section{Chapter 16}

\section{Systematic Errors}

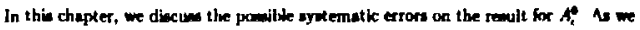
shall wee from he aize of the etrors derived betow, the dominant aystenutic errors

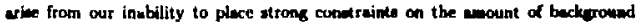

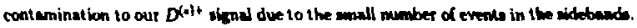
Let wh turn now to the presentation of the uystematic errors, which will be aumarinad in Tuble 16.5 .

\subsection{Systematic Errors Related to the Background}

This wection collects all of the nystematic ertors amocinted with our inprecine knowiedge of the properties of the buckeround sumple.

\subsubsection{Determination of the Backgraund Fraction}

The frections of signal and RCBG $\left(f_{\text {man }}\right)$ in the cignal regions were calculated in Section 13.3 by adjustiag the background and sianal ahapes in the signal and stieband regions until the two combined shapes fit the observed date. This celculation suffers from two problerne: low beckeround atatistice and incorrect MC velues for weverel of the branching fractions and production croes sections of the $D$ mesums ued in the andysis. To minimize the effects of these uncerteintion, wo wed the coly the ahapes 


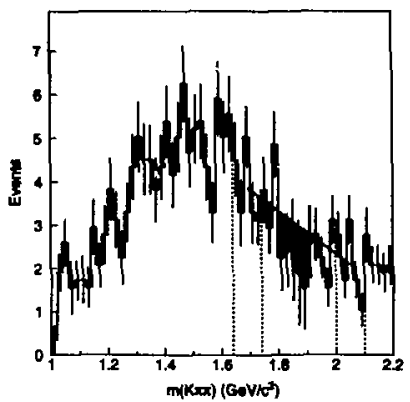

Figure 16.1: The MC buckground distribution for the $D^{+}$analysis. The sideband regions are those between tine deslued lines. The sloping tine shown is drawn between the menn values of the number of events in ench sideland, with the asumed starting and endiag points being the centers of each of the sideband regions. The grey region indicates the amount by which this method of determining tbe backgound shape overestimates the number of background events in the signal region.

of the signal and baskground samples and allowed thit relative nonalizations to Hucturte. Even this was not sufficient in the case of the the $D^{+}$sumple, as the cross exction for the production of $D^{+}$in charan events was too low in the MC ainulation, so that $35 \%$ more pure $D^{+}$signal needed to be added to the distribution to obtain renenuble agrement.

We can estinate the error on these calculations in a number of independent ways. First, there bs the simple statistical error on the number of events in the sidebands for both MC and date. This amounts to $13.1 \%$ of $f_{\text {manc }}$. Second, we can we the mesured eymenctry in the sidebends with no sigal subtracted, $12 \%$, and mume that this comes entirely from signal, so that the fraction of RCBG in the sidebands, 
$f_{\text {mend }}^{\text {s. }}$ in given by

$$
0.12=\left(1-f_{\text {ced }}^{S g}\right) \times 0.67 \text {. }
$$

This impiles that $f_{\text {mer }}^{g D}$ is $(6.4 \pm 13.5) \%$ bee then what we calculate with the other method. Thirdly, we can compare the background ahepen from the MC to the observed data dintribution. For the $D^{\text {ot }}$ modes, we can use the MC beckgrowed unpe to project the number of dats events in the "dend" recion betwoen the alow and sideband regions of the $\Delta m$ plot. Any excen can thea be attributed to an error in $f_{\text {ncer }}$. We find a difinence of $(4.5 \pm 21.0) \%$ in these two decay nodes. We can

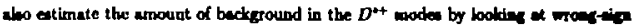
three truck combination, where the $D^{n}$ is formed unine two enes-iga tracke and is then combined with en oppositely-charged tract at the dow pion candilete Unfortunately, atatiatica on these combinations are limited in the data; thie lachaique result in en eatimate of $\delta f_{\text {mac }}=41 \pm 23 \%$. For the $D^{+}$mode, these are a mobor of options:

- the prosedure described in Section 13.3 give $f_{m=10}=0.16$

- we can tube from the MC the difiereace in a linenr extrapolation of the backeroundonly aldeband populations underneath the $D^{+}$man peak and the number of events that actually are under the poak. Thin situation be shemn in Fisure 16.1. The linear extrapolation overestimates the number of buckground ovents in the $D^{+}$sumple by $(23 \pm 8) \%$ if the MC can be trusted at thin level.

- We have aleo compared the predictions of a fit to the shepes of the backeround and signal with a tirgle gavenien plus a third-order polyoomid. The fit for the date is shown in Figure 16.2n and for the MC in Figure 16.26". This lit to the MC gives an extimate of 101 aignal events in the poik, which actually contain $145 \mathrm{D}^{+}$decays. The fit is apperently pulled high by fluctuntions just outeide the mas peak gaumien, and the amount of aignal is underentimated by $43 \%$. Ulin: the rame (poenibly flawed) technique for the data, we find that, when corrected for the amount of aignal in the sidetendn, we obtain an etimate that the man

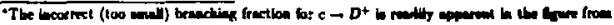
twe MC. 
Table 16.1: The number of double-tazsed $D^{0}$ decays in data and MC

\begin{tabular}{|ccc|}
\hline Decay Mode & for (DATA) & for (MC) \\
\hline$D^{2+} \rightarrow \pi^{+}\left(K^{-} \pi^{+}\right)$ & $0.284 \pm 0.057$ & 0.272 \\
$D^{++} \rightarrow \pi_{-}^{+}\left(K^{-} \pi^{+} \pi^{0}\right)$ & $0.252 \pm 0.046$ & 0.213 \\
\hline
\end{tabular}

peak is $70 \%$ signal, or $f_{\text {nce }}=0.3$ (which can be compered with the calculated value of 0.16 ). Since we know this technique ent toward underedimating the signal fraction by a large amount, this can be conaidered ae an relatively hand upper limit un $f_{R C A C}$ for the $D^{+}$decay mode. This metbod merven then wo more of a cross check than an estimation of the size of the variation in the background fraction.

Uning the stutistical errors on these messurentents. we take a meighted averase over all of the different techniques to obenin the error on the beckground fraction of the entire rh arm sumple of $\delta f_{\text {mas }}= \pm 18.8 \%$, or $f_{\text {mas }}=0.239 \pm 0.05$.

An independent way to check the asumed beckground and sigual fractions in the case of the $D^{0}$ decay modes is to examine the fraction of overlap between those events selectod in the kinematic vs. tile decey length andyris. The metbods are sulficiently different in their demands on the signal events that wan hypothesize that only real signal events will actually be "double-tagged" by being selected by both rets of cuth. Let us first consider the double tag fraction for fnund in the dats compared with that from the MC with no modifications. These numbers are presented in Table 16.1.

Note that the $M C$ events have a siguificantly lower fraction of double tag. Wo can we the MC simulation to check the hypotheals that only signal events are doubletagged. From the MC, we find that no bechground evenus are double-tagged, and the fraction of signal esents that double tag ( $f_{\text {of }}^{\text {fall }}$ ) are 0.383 for the $K \pi$ mode, and 0.350 lor the $K \pi \pi^{0}$ mode. Assuming that only algnal events double tag, we have

$$
f_{D T}=f_{D} f\left(1-f_{\text {MCno }}\right) \text {. }
$$

The double tag fraction is then directly proportional to the fraction of beckgound in 


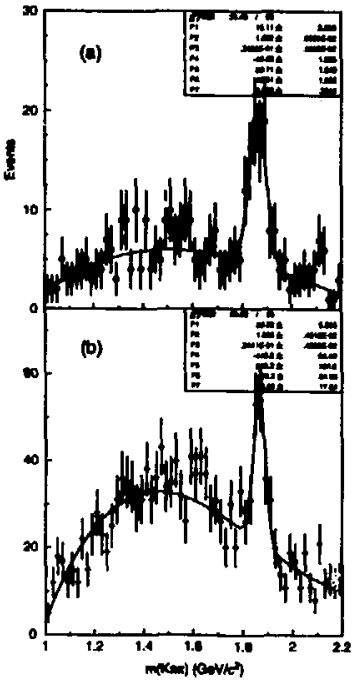

Figure 16.2: A third order polynomial beckeround fit to the $D^{+}$signal and beckeround for ( $a$ ) the date, and (b) the MC. The firs three fit paramotors are thoee lor the gaussien. Note that the width of the $D^{+}$peak in the data is approxingately $40 \%$ las gar than that in the MC; the full extent of the poak atil lim woll within our siand redion of $K \pi \pi$ man. 
Tuble 16.2: The number of docble-tapged $D^{0}$ decnys in data and MC \& frer correction

\begin{tabular}{|ccc|}
\hline Decay lode & $f_{D T}$ (DATA) & $f_{D T}$ (MC) \\
\hline$\left.D^{+4}-\pi^{+} K^{-} \pi^{+}\right)$ & 0.284 & 0.284 \\
$D^{++} \rightarrow \pi^{+}\left(h^{-} \pi^{+} \pi^{0}\right)$ & 0.282 & 0.261 \\
\hline
\end{tabular}

the signal redon, making this a very mennitive test of our measure of the beckgtound

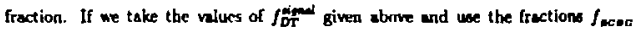
derived from our procedure o normalization adjustment, we obsaln the values of Table 16.2. The values support our conclusions on the relative amounts of signal

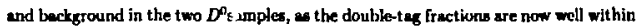
$1 \sigma$ errors of the data.

Assuming the allowed variation in the buckground frections for the three decay modes in the likelibood fit lear's to an overall error duc to this source of 0.044 on $A_{c}^{0}$. Changing of the background fractions modifies the swount of obscrved asymmetry that is assumed to arise from cham and b decays; an $18 \%$ change in the background fraction thould bave a large effect on the charm asymmetry.

\subsubsection{The Background Asymmetry}

When the esymmetry due to the presence of a small number of signal events in the sidebend regions is suberweted, we obtain a net sideband asyrnmetry of $+0.05 \pm 0.10$. For the central value, we heve asumed that the background asymmetry is zero, and have taken the $1 \sigma$ statistical deviation, 0.15 , as an upper limit on the background asymmetry. This yields $n$ char ge in $A_{c}^{a}$ of 0.044 when included in the likelihood fit. Since the beckpround fraction in any of the samples is of the order $20 \%$, wo would expect that allowing a larger beckground asymmetry should have a non-negligible effect on the charn aymmetry: 


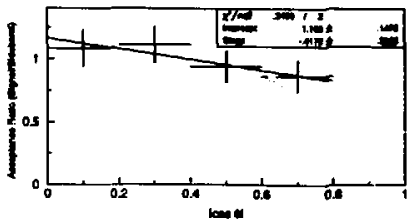

Figure 16.3: Comparien of signal and sideband acceptances, obtatned by dividine the normalized diatributions of the number of events us. loosef for the ninan and sideband data samples. Tho ft parameters are aloo showr.

\subsubsection{Back round Acceptance}

From Figure 13.20, it can be seen that the accepennce for moonetructed $D$ berin to fall of around $|\cos 0| \sim 0.65$. In an altempt to chect whother the exceptence for Lackgound events has the sane behuviour, we plot in Fizure 16.3 the ratio of the signal to background acceptance as a function of $|c 0 s|$. A the ratio in aymantric abo't cve $\theta$ In 0 , we have folded the distribution over to obtain amaller atimical errors. A simple linear fit yields

$$
R_{\operatorname{man}}=-.41 \cdot \cos \theta \mid+1.16,
$$

whic Rerer $_{\text {is }}$ is the ratio of signal to beckeround acceptance. We asurned for the central value of $A_{c}^{0}$ that the signal and beckground acceptances were the reve. If the backgrousd fruction in the fit is allowed to depend on $|\cos \theta|=$ specised by the above expuation, the value of $A_{c}^{0}$ changes $L_{j} n .045$. This error expected to be one of the largest systematic errors, $\omega$ it is dificult en eatablinh that the sigal and buckground acceptances are identical. Since the mymaetry in much leren at

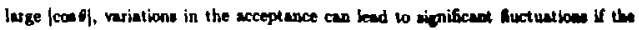
background fraction is allowed to change where the event wiples are largat. 


\subsubsection{Background $x$ Dintribution}

Our seneitivity to statiotical fluctuations in the enersy spectrun of the radom combinator; - buckground is checked by performing the andysin with the probubility functios $F_{\text {sig }}$ derived from the Monte Carb be zkground inateod of the dete from the adeband regions. To do this, the $x$ distribution from the MC sidebands is ued inteed of that foom the data. The saroe procedure presented in Section 14.2.2 iw then ued to reculculate the ratios $N_{\text {signod }}(x) / N_{\text {matprosed }}(x)$ for each of the three decay modes. This results in a change of 0.039 in $A_{c}^{0}$, which is certainly conciatent with the Ketistical errors on the number of evencs in the sidebands.

We can also cheak the sive of this variation by ming the MC incted of the data for all of the ahapes including $\boldsymbol{N}_{\text {wet }}$, the total shape of aigeal and backeround. Following the same procedure, we obtain a change in $A_{c}^{\mathrm{u}}$ of 0.0G4. We know that this in an overestimate cI the error, sirce the MC event ample hes a amaller ratio of oignal to background than is observed in the cista.

As another check, we can ignure the presence of any amount of aignal in the sidebands and just use the date distribution to calculate $N_{\text {sipmed }}(x) / N_{\text {mandend }}(x)$. This results in a change of 0.019 in $A_{c}^{\mathrm{g}}$. We would expect that this would be smaller than ueing the MC sideband shape, as the anount of signal in the sideband regions is andl, $w$ the net result of the signal subtraction is a minor change in the distribution.

\subsection{Systematic Errors Related to the Signal}

We collect in this extion those systematic errors that result from the various amumptions made about signal production rates, composition, fregunenation functions, and aymmetries.

\subsubsection{Determination of the Signal Componition}

One of the potentid cources of error in this analyin is the determination of the fraction of eveots from bo $c$ decays that popelate the rigul region. One dificulty in deriving these quantitice is that the branching fractione for $c \rightarrow D^{+}$and the ratio 
Table 16.3: Exiating menuremente of $D^{+}$and $D^{+}$production in $Z$ docises.

\begin{tabular}{|cc|}
\hline Source & $F_{C} \cdot R_{-D^{*}} / F_{c} \cdot P_{C-D^{*}}$ \\
\hline DELPH(68) & $1.119 \pm 0.304$ \\
ALEPH[67] & $1.119 \pm 0.160$ \\
OPAL[6O] & $1.000 \pm 0.201$ \\
OPAL[200] & $1.367 \pm 0.168$ \\
SLD MC & 0.946 \\
\hline Soucce & $F_{b} \cdot P_{L \rightarrow D^{+}} / F_{c} \cdot P_{C \rightarrow D^{+}}$ \\
\hline DELPHI[68] & $1.119 \pm 0.437$ \\
SLD MC & 1.530 \\
\hline
\end{tabular}

of the production rates of vector mesoes to that for preudoecalar mesom in charm decays ued in generating our MC manple do wot corrempond to the menund wim for these quankities.

If male the ulmpte argument that tbe phyical procenom isvolved in produciax charmed moons in the breakup of 6 badrones should be similer to that which occurs in the randon peiring of quark during fragnentelion, we would expect that the relative fractions of $D$ meson from these source in the entirc bedronic event manple to be eiven by $\Gamma\left(Z^{0} \rightarrow b \bar{b}\right) / \Gamma\left(Z^{0} \rightarrow \alpha^{0}\right) \approx 0.22 / 0.17 * 1.29$. Several mearuremente of $D^{\text {H+ and }} D^{+}$production have been performed by the LEP experiutents. They typically reasure the combined product $F_{b} \cdot R_{\rightarrow D} / F_{\mathrm{c}} \cdot P_{C \rightarrow D}$, where $F_{\mathrm{V}}=\Gamma\left(Z^{A} \rightarrow \mathrm{Cl}\right)$, and $P_{q \rightarrow D}$ is the branching fraction for quark type $q$ to the $D$ mean of choice. Their reaults are summerised in Table 10.3, alons with the value und by our MC airulation. The values in the MC were adjurted in the tuning of the b phyaca modeling described in Chapter 4 and were the realt of a conecious effort to make the modeling of b dacays n accurate a powible. Since changing the MC is not powible at this stape, we can only eatimate the efiects of the viriotion of thene production crom-nection. To aid this diecuedian, we introduce beso the peranete $R_{D} \equiv F_{b} \cdot R_{b \rightarrow b} / F_{c} \cdot P_{c \rightarrow D}$.

The redevane quantity to the analyain in not actually the production croses action, but the frection of the events from each quark avor that pan the cuts, $f_{g \rightarrow 0}=$ 
Table 16.'. The variation of us for difterent values of $F_{b} \cdot R_{-b} / F_{n} \cdot P_{\mathrm{c} \rightarrow b}$.

\begin{tabular}{|lcc|}
\hline Ascumption & $w_{b}$ & Change $(\%)$ \\
\hline Default & 0.254 & - \\
$R_{D^{*}}=R_{D^{*}}=1.12$ & 0.267 & +5.3 \\
$\boldsymbol{R}_{D^{*}}=R_{D^{+}}=1.29$ & 0.294 & +15.9 \\
$\boldsymbol{R}_{D^{*}}=1.54$ & 0.329 & +29.8 \\
$\boldsymbol{R}_{D^{*}}=0.946$ & 0.237 & -6.5 \\
\hline
\end{tabular}

$F_{q} \cdot P_{q \rightarrow 0} \cdot \varepsilon_{q}^{\text {and, whe }} \varepsilon_{q}$, is the probability that $D$ mesons produced by the quark $q$ pasa the selection cuts. The quantity $\omega_{\mathrm{g}}$, the fraction of the signal from quark type $q(q=a, b)$, was introduced in Chapter 13 and can now be definal ws, e.g.,

$$
\omega=\frac{f_{b-D}}{f_{c \rightarrow D}+f_{b \rightarrow D}} .
$$

The uncertainty in the relative production cross sections can then be re-expressed as an uncertainty on w. If we calculate wh from our default $M C$ xilutilation, we obtain $w_{0}=0.254$ as the averafe for the ent i-n charm sample. Table 16.4 gives the variation in $\omega_{b}$ for various tisumptions on the relative production croses sections $R_{b}$.

The first assimption is that both values of $R_{p}$ are equal to the worlel average value. The second corrasponds to our naive guess of the relative cross sections. The third and fourth vuriations giniply assume that $\boldsymbol{R}_{D^{*}}=\boldsymbol{R}_{D^{+}}$and take the two different MC values as the possible extrema. We have chosen to take the largest possiblo variation as our systematic error, to we use $\delta w_{b} / w_{b}=0.30$.

Using this variation of $w$ in the likelihood fit yields an overall error on $A_{c}^{0} 0.005$ from this uncertainty. The insensitivity to the frection of b events in the signal is due to the near identical values of $A_{b}$ and $A_{c}$ for $D$ s after the dilution due to $b$ inixing.

An independen erosecheck can be periornied on the determisantion of the relativo fractions of signal and background and the relative fractions of $b$ and $c$ in the signal for the case of the $D^{+}$decay mode. Figure 13.18 shows a broad bump of signal evonts to the left of the inein $D^{+}$mass peak. If our estimate of the signal fraction in the sidebands is correct (it depends on both the addition of $35 \%$ of $\mathrm{c} \rightarrow D^{+}$production 
and the aideband signal normalization procedure), the fitted asymmetry in the redon should be non-zero. From the ratio of nignal to background in the region from 1.0 $\mathrm{GeV} / c^{2}<m(K \pi \pi)<1.04 \mathrm{GeV} / c^{2}$ which lies directly outside the nidebend reqton, we calculate that the measured symmetry abould be 0.26 . A four-bin fit yield a value of $0.26 \pm 0.18$, which is certainly coneistent. This inplies that our treatment of the signal and backgrounds in the $D^{+}$cae is at leat welf-condistert.

\subsubsection{Variation of the Value for $A b \rightarrow D$}

In Section 14.2.1, we discused the procedure by which the value of $A_{b \rightarrow D}$ used in the likelihood fit, $A_{b \rightarrow D}=0.64 \pm 0.11$, wes determined. Allowing this value to fluctuate by the allowed error reaults in a 0.020 rhange in $A_{c}^{0}$. As $A_{b \rightarrow D}$ moves away from being identically equal to $A_{c}$, some fluctuation should result. The size of this error is about what is expected, given the niatively sinal fraction of b events in the signal anmple.

\subsubsection{Fragmentation Function Variation}

A number of checks can be made on our formulation of the fragmentation functions which wes presented in Soction 14.2.2. Firal, we cun consider varietions in the "hardness" of the fragmenation functions for $b$ and $c$ decays besod on the Uncertainties on the measurements of the fragmentatlon functions themselve from the LEP collaborations[123]. The central values used in our MC sample are $\epsilon_{e}=0.06$ and $\epsilon_{4}=\mathbf{0 . 0 0 6}$, where $c_{\text {g }}$ is the parameter in the Peterson Eragmentation function that controls the shape of the quask energy epectrum. To eccount for the uncertsintien in the fragnentation functions, we generated samples of cherm and $b$ decaya to $D^{\text {at }}$ and $D^{+}$mesons at values of $\epsilon_{c}=0.035,0.095$ and $\epsilon_{b}=0.0035,0.011$, which are the range of uncertainties recommended for use in studies of the electroweak physics of heavy Quarks by the LEP working group on this topic. As an example of the different shapes that are obtained, Figure 16.4 showa the nomina, hard, and soft fragmentation shapes for $c \rightarrow D^{+}$decays. For each chenge of fragmenation paremeter, the function $d_{c \rightarrow D}$ and $d_{b \rightarrow D}$ are refit, and the new parametrizations are input into the likelihood fit. Because of the difference in energy spectrum, the values of $N_{\text {signil }}(x) / N_{\text {mapromer }}(x)$ 


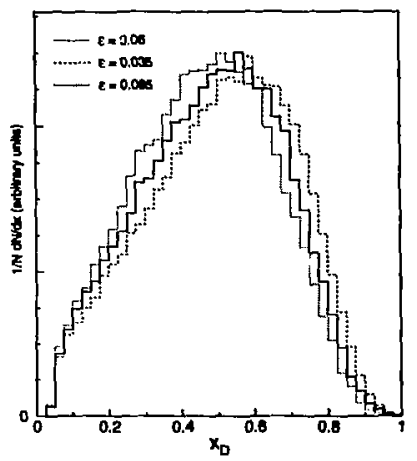

Figure 16.1: 'The MC distributions of $x_{b \rightarrow p}$ for nominal $(c=0.06)$, hard $(\epsilon=0.035)$, and soft $(c=0.095)$ clarm fragratentation.

are also adjusted accordingly in order to perform this check. This modification to the likelihood function results in maximal changes of 0.025 and 0.007 to $A_{c}^{0}$ for the changes in $\epsilon_{e}$ and $\epsilon_{b}$, respectively. We expect a relatively large dependence on the nesumptions of fragencitation functions for the cham decayn, since the $x_{D}$ distribution is the only tool we use to separate charm decays fron $c$ and $b$ events. Since $c$ events contribute by far the largest fraction to the signal, the aize of this variation is commensurate with their weight in the fit. The change in $d_{b}, 0$ for different values of $\epsilon$ is relatively small; since the $D s$ in $b$ decay are produced in the decay cascade, changes in the fragmentation function produce only second-order effects on the $D$ energy distribution. Modifying the fraction of the charm evento that pass the cuts $\left(\omega_{c}\right)$ baned on the changes of the framentalion functions when the neap enerpies are 
shifted yieide amuller changes to $A_{c}^{0}$ then the above values.

We heve chowen to take the meximal enors a the uncerteinty due to our traprecive knowledge of the fragmentation function.

To check our mumption on the shepen of the fregmentation functions, we can obeerve the variation on the value of $A_{c}^{0}$ when we change the fit inputs. For the $c$ events, we can replace the parametrized fit of the Petereon function' with a normalined bin-valued function besed on the geterated diatribution. This will not treat eveme that lie on the rapidly changing parts of tbe epectrum properiy, but it given ve some eatimate of our nensitivity to the exwct abape of the diatribution. For the $b$ eventu, we can replace the OPAL 6tting function with a simpte, exponentid function times a gauswien. This yiekd acceptable fits in the regions above our cuts on $x_{\mathcal{D}}$. The variations on $A_{c}^{0}$ due to thene modifications are 0.023 and 0.005 for the $c$ and 6 changes. We add these in quedrature to obtain an errot duc to our arumptions on the fitting ohepe.

\subsection{Uncertainties from Outside Parameters}

Finelly, we have the uncertainties arising fron the other inputs to the fitting function.

\subsubsection{Uncertainty on $A_{e}$}

As mentioned in Section 14.2, we have chosen to use $A_{q}=0.1617 \pm 0.012$. The resulting clange in $A_{e}^{0}$ due to allowing $A_{e}$ to very over this range is 0.003 . This bears out our statement that the results of the likelihood fit abould yield a value of $A_{c}^{0}$ that is atatisticully insensitive to $A_{e}$, w this method should be equivalent to forming the double asymmetry of Eq. 2.72 which is, by definition, independent of the initial-state coupling.

\footnotetext{
The It pullity is at perticularly nood.
} 


\subsubsection{Polarization Uncertainty}

Changing the value of the meusured polarization by its error yields a variation of

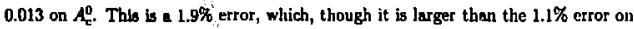
the polarization value, is comparable to our expectations, since the error on $A_{c}^{0}$ is directly proportional to the polarization error.

\subsubsection{Uncertainty in the Value of $\alpha_{a}\left(M_{z}^{2}\right)$}

For the QCD radiative corrections, a central value of $\alpha_{f}\left(M_{2}^{3}\right)$ must be assumed. We have taken the Particle Data Group value of $\alpha_{0}\left(M_{z}^{2}\right)=\mathbf{0 . 1 1 9}$ as averaged from measurements using jet rates and assigned a generous error of 0.02 in our calculation of the radiative corrections. This is justified, as estimates of the theoretical errors on

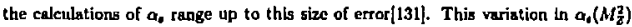
results in a 0.005 change in $A_{\mathrm{a}}^{\mathrm{O}}$.

\subsubsection{Uncertainty in the $\mathcal{O}\left(\alpha_{4}^{2}\right)$ QCD Correction}

The errors reported in Chapter 15 for the $\mathcal{O}\left(a_{n}^{2}\right)$ QCD corrections are dominuted by uncertainties in the quark masses as input to the QCD calculations for the size of the corrections. We also include the crror on $\alpha_{,}\left(M_{2}\right)$ aind on the relative rates of the production of heavy quark events from gluon splitting $\left(f_{D_{B}}=\mathbf{0 . 4 7} \pm 0.25\right.$ ), to obtain e total relative error of $1 \%$ on thls correction. The corresponding variation of $A_{c}^{0}$ is 0.007 .

All of the systernatic errors are summarized Table 16.5.

We now turn to a discussion of these results and $\mathrm{n}$ comparison with other existing measurements. 
Table 16.5: Contributions to the estimated systematc error on $\boldsymbol{A}_{\varepsilon}^{a}$

\begin{tabular}{|c|c|c|}
\hline SOURCE & RANGE & ERROR \\
\hline 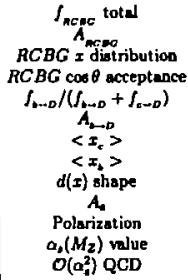 & $\begin{array}{c}0.239 \pm 0.045 \\
+0.05 \pm 0.10 \\
\text { sideband vo MC } \\
\text { sideband vo sigma } \\
0.254 \pm 0.076 \\
+0.64 \pm 0.11 \\
0.035 \leq \epsilon_{2} \leq 0.095 \\
0.003 \leq \epsilon_{\mathrm{b}} \leq 0.011 \\
\text { bing ws. fit } \\
0.1617 \pm 0.012 \\
63.0 \pm 1.1 \% \\
0.118 \pm 0.002\end{array}$ & $\begin{array}{l}0.038 \\
0.044 \\
0.039 \\
0.045 \\
0.005 \\
0.020 \\
0.025 \\
0.007 \\
0.023 \\
0.003 \\
0.013 \\
0.005 \\
0.007\end{array}$ \\
\hline Total & & 0.005 \\
\hline
\end{tabular}




\section{Chapter 17}

\section{Conclusions}

\subsection{Final Result}

Including the systematic errors discussed in the previous section, we arrive at a fiual result for $A_{c}^{0}$ of

$$
A_{\mathrm{c}}^{0}=0.73 \pm 0.22 \text { (stat) } \pm 0.10(\text { syst })
$$

\subsection{Comparison with Existing Measurements}

We present in Figure 17.2 a comparison of the results from messurements of $A_{c}^{a}$ nusde at SLD with thaxe from the LEP collaborations; their measurements of $A_{F n}^{f}$ have boen divided by their value for $A_{c}$ taken from their measurernents in the lepton sector: $A_{e}=0.1453 \pm 0.0057$. Using the world-average value of $A_{e}$ only shifts the scale slightly. We can immediately the added statistical power afforded by the polarized asymmetry. The presence of a large raw asymmetry in the polarized data allows our measurements to compete on equal footing with those from LEP using data samples conteining forty times more $Z^{0}$ events. The total error on $A_{e}$ from the $D$ andysis is actually smal]er than that for all of the other measurements excespt the lepton result from OPAL and the ALEPH $D^{*}$ analysis. 


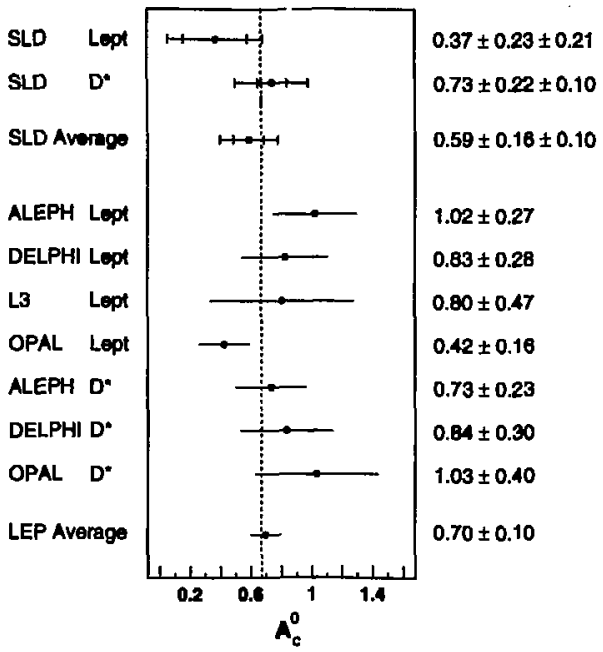

Figure 17.1: A comparison of the workd's measurements of $A_{\mathrm{c}}^{0}$. The results of this thesis and the otber SLD result obtained by fitting the high $p, p_{1}$ Septon spectrum have been everaged to quote an "SLD A verage" value. (See text.) The vertical line is drawn at the standard model value of $A_{\varepsilon}^{0}=0.67$, suming $\sin ^{2} \theta_{\phi}^{\prime \prime}=0.234$. 
The vertical line drawn on the figure is the standard model expectation for $A_{\varepsilon}$ souming $\sin ^{2} \theta_{W}^{\prime \prime \prime}=0.234$. Our result is exrtainly consistent with the standard model within our etrors.

\subsection{Determination of $\sin ^{2} \theta_{W}^{e f f}$ from $A_{c}$}

As mentioned in Chepter 2, the value $A_{c}$ does have a strong dependence on the value of $\sin ^{2} \theta_{\psi}^{\prime \prime}$ :

$$
\frac{d A_{c}}{d \sin ^{2} \theta_{w}}=-3.42 \text {. }
$$

Given, this, we can invert the relationship

$$
A_{c}=\frac{2 v_{c} a_{c}}{v_{c}^{2}+a_{c}^{2}}=\frac{2\left[1-\frac{a^{2}}{3} \sin ^{2} \theta_{W}^{c /}\left(M_{Z}^{2}\right)\right]}{1+\left[1-\frac{n}{3} \sin ^{2} \theta_{W}^{c J}\left(M_{Z}^{2}\right)\right]^{2}},
$$

solving for $\sin ^{2} \sigma_{W}^{f f}$. This yields the result

$$
\sin ^{2} \theta_{W}^{\alpha f J}=0.212_{-0.130}^{+0.085} \text {. }
$$

The reason for the agymmetric error can be seen in Figure 17.2, where the value of $\sin ^{2} \theta_{W}^{\prime \prime}$ is plotted aguinst values of $A_{c}$. Because of the functional dependence of the couplings, the lower bound is weaker than the upper bound. We can compare this result to that derived from $A_{L A}[\mathbf{4 7}]$

$$
\sin ^{2} \theta_{W}^{\prime J}=0.2294 \pm 0.0010
$$

It is easy to see that this measurument will certainly not ever compete with $A_{L R}$ for a measurement of $\sin ^{2} \theta_{w}^{\prime J}$. Is is, howuver, a unique measurement of the $Z \propto$ coupling.

\subsection{Possible Improvements}

It is clear from the discumion of this measurement that event statistics are the limiting Cactor for this analysis. More data brings with it more analyzing power for the asym. metry as well as more statistics in the sideband regions whoee uncertainties dominate 


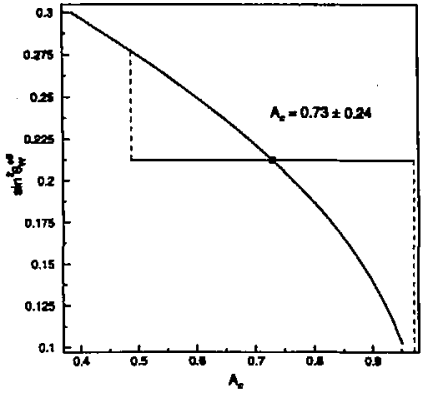

Figure 17.2: The dependence of $\sin ^{2} \theta_{W}^{\prime \prime}$ on $A_{c}$, sbowing the results of this meneurement with combined atatistical and systematic errons.

the systematic error on our measurenent. To this end, we aro at work on en incluoive method of tagging $D^{*+}$ decays using a partial reconstruction of the $D^{0}$ vertex. 5ince the vertex flight direction can be measured extremely well by the precision tracking of SLD, the $D^{*+}$ direction can be estimated with stall errors. The $\pi_{4}^{+}$from the $D^{\text {t+ }}$ decey will have extremely low $p_{\perp}$ with respect to the vertex flight direction; tracks of this type will form a peak at $p_{\perp}^{2}=0$ in the distribution of transverse momenturn of all tracks with respect to the vertex flight distances. With sujtable kinematic selection criteria on the three-track combinstions, a clean sample of $D^{++} \rightarrow D^{0}$ decaya can be sclected without reconstructing the $D^{0}$. We estimate that the efficiency for findine the two-prong vertex in these decays to be about $40 \%$, 30 this tasging method abould be much more efficient than the standard exclusive reconstruction.

In addition, we cen also use the information provided by the preciaion vertex to help better distinguish bet ween $b$ and $c$ decays in the sumple. Onco mont atatiatica 
are cccumulated with the SLD detector, the errors due to $b$ contamination may well be comparable to thooe introduced by the beckgrounds. If we add to the likelihood function the probability distributions for vertex pointing, the proper lifetime of the decay, and any other variable we can derive that allows better separation of $b$ and $c$ events, we should be able to reduce the systematic errors ever further. The new SLD vertex detector with hundreds of thousands of $Z^{0}$ decays should ensble the world's best messurement of $A_{c}$ using these techniques.

If we wish to reach a precision of $\sim 1-2 \%$ on the mesurement of $A_{c}$, which is the level at which deviations due to "new physics" could arise, this more efficient tog will be cruciel. A simple extrapolation of the present result to $1 \times 10^{8} Z^{0}$ decays only reduces the statistical error to $5 \%$. If we wish to approach a $1 \%$ statistical error, we must somehow increase the selection efficiency for charm events by a factor of $\mathbf{2 5}$ without substantislly worsening the sasple purity. This will require the oddition of mare exclusive modes through the use of $\pi^{\circ}$ reconstruction, the use of the SLD CRID to tag $D^{0}$ decays without requiring the slow pion, and as many other techniques as we can create. 


\section{Appendices}




\section{Appendix A}

\section{Efficiency Matrices for $R_{3}$ Unfolding}

Here, we list the four $3 \times 3$ matrices used to unfold the perton-level 3-jet rates from the lasged aumples. We give the matrices for each of the three run periods separateily. A semple matrix of atatiotical errors for the 2-jet to 2-jet toger like

$$
\delta\left(\varepsilon_{2-2}^{i_{1}}\right)=\left(\begin{array}{lll}
0.0023 & 0.0035 & 0.0011 \\
0.0015 & 0.0011 & 0.0033 \\
0.0001 & 0.0012 & 0.0035
\end{array}\right) .
$$

PreVeto:

E:

$$
\begin{aligned}
& \varepsilon_{2 \rightarrow 2}^{i j}=\left(\begin{array}{lll}
0.4077 & 0.1938 & 0.0203 \\
0.1173 & 0.3169 & 0.2279 \\
0.0006 & 0.0177 & 0.2797
\end{array}\right) \varepsilon_{3 \rightarrow 2}^{i j}=\left(\begin{array}{lll}
0.1881 & 0.1024 & 0.0179 \\
0.0650 & 0.1355 & 0.1140 \\
0.0004 & 0.0070 & 0.1032
\end{array}\right) \\
& \varepsilon_{3 \rightarrow 3}^{i j}=\left(\begin{array}{lll}
0.2466 & 0.1385 & 0.0260 \\
0.0909 & 0.1911 & 0.1787 \\
0.0009 & 0.0096 & 0.1364
\end{array}\right) \varepsilon_{2 \rightarrow 3}^{i j}=\left(\begin{array}{lll}
0.0321 & 0.0175 & 0.0023 \\
0.0112 & 0.0230 & 0.0186 \\
0.0000 & 0.0012 & 0.0183
\end{array}\right)
\end{aligned}
$$


50:

$$
\begin{aligned}
& \varepsilon_{2 \rightarrow 2}^{j j}=\left(\begin{array}{lll}
0.4178 & 0.2025 & 0.0206 \\
0.1201 & 0.3198 & 0.2327 \\
0.0006 & 0.0182 & 0.2890
\end{array}\right) \varepsilon_{3 \rightarrow 2}^{U}=\left(\begin{array}{llll}
0.1874 & 0.0025 & 0.0174 \\
0.0034 & 0.1390 & 0.1219 \\
0.0003 & 0.0067 & 0.1074
\end{array}\right) \\
& \varepsilon_{3 \rightarrow 3}^{i j}=\left(\begin{array}{lll}
0.2454 & 0.1408 & 0.0238 \\
0.0912 & 0.1928 & 0.1688 \\
0.0010 & 0.0008 & 0.1407
\end{array}\right) \varepsilon_{2-3}^{i j}=\left(\begin{array}{lll}
0.0232 & 0.0105 & 0.0015 \\
0.0072 & 0.0183 & 0.0112 \\
0.0000 & 0.0006 & 0.0005
\end{array}\right)
\end{aligned}
$$
P:

$$
\begin{aligned}
& \varepsilon_{2 \rightarrow 2}^{i j}=\left(\begin{array}{lll}
0.4181 & 0.1963 & 0.0201 \\
0.1174 & 0.3224 & 0.2304 \\
0.0005 & 0.0192 & 0.2890
\end{array}\right) \varepsilon_{3 \rightarrow 2}^{U}=\left(\begin{array}{lll}
0.1701 & 0.0085 & 0.0139 \\
0.0563 & 0.1249 & 0.1007 \\
0.0002 & 0.0046 & 0.1103
\end{array}\right) \\
& \varepsilon_{3-j}^{i j}=\left(\begin{array}{lll}
0.2620 & 0.1450 & 0.0239 \\
0.0954 & 0.2033 & 0.1724 \\
0.0010 & 0.0111 & 0.1462
\end{array}\right) \varepsilon_{3 \rightarrow 3}^{i j}=\left(\begin{array}{lll}
0.0244 & 0.0116 & 0.0014 \\
0.0079 & 0.0103 & 0.0127 \\
0.0001 & 0.0007 & 0.0115
\end{array}\right)
\end{aligned}
$$

P0:

$$
\begin{aligned}
& \epsilon_{2-2}^{i j}=\left(\begin{array}{lll}
0.4166 & 0.1963 & 0.0194 \\
0.1162 & 0.3208 & 0.2275 \\
0.0005 & 0.0193 & 0.2887
\end{array}\right) \epsilon_{3 \rightarrow 2}^{y}=\left(\begin{array}{llll}
0.1619 & 0.0636 & 0.0128 \\
0.0534 & 0.1179 & 0.1020 \\
0.0002 & 0.0046 & 0.1060
\end{array}\right) \\
& \varepsilon_{3-3}^{i j}=\left(\begin{array}{lll}
0.2697 & 0.1486 & 0.0248 \\
0.0986 & 0.2109 & 0.1805 \\
0.0011 & 0.0108 & 0.1509
\end{array}\right) \epsilon_{2 \rightarrow 3}^{j j}=\left(\begin{array}{lll}
0.0266 & 0.0138 & 0.0019 \\
0.0083 & 0.0209 & 0.0139 \\
0.0001 & 0.0009 & 0.0125
\end{array}\right)
\end{aligned}
$$

D:

$$
\begin{aligned}
& \varepsilon_{2 \rightarrow 2}^{i j}=\left(\begin{array}{lll}
0.4213 & 0.2016 & 0.0190 \\
0.1191 & 0.3258 & 0.2295 \\
0.0005 & 0.0185 & 0.2919
\end{array}\right) \\
& \varepsilon_{3 \rightarrow 3}^{i j}=\left(\begin{array}{lll}
0.2589 & 0.1449 & 0.0276 \\
0.0991 & 0.1962 & 0.1894 \\
0.0012 & 0.0104 & 0.1470
\end{array}\right) \varepsilon_{3.02}^{i j}=\left(\begin{array}{lll}
0.1713 & 0.0898 & 0.0180 \\
0.0578 & 0.1293 & 0.1058 \\
0.0003 & 0.0067 & 0.0910
\end{array}\right) \\
& \varepsilon_{2-3}^{i j}=\left(\begin{array}{lll}
0.0210 & 0.0101 & 0.0019 \\
0.0067 & 0.0157 & 0.0139 \\
0.0000 & 0.0003 & 0.0093
\end{array}\right)
\end{aligned}
$$


G:

$$
\begin{aligned}
& t_{2 \rightarrow 2}^{i j}=\left(\begin{array}{lll}
0.4030 & 0.1868 & 0.0153 \\
0.1074 & 0.3142 & 0.2088 \\
0.0004 & 0.0185 & 0.2891
\end{array}\right) \quad t_{3 \rightarrow 2}^{i j}=\left(\begin{array}{lll}
0.1348 & 0.0715 & 0.0099 \\
0.0440 & 0.0980 & 0.0770 \\
0.0002 & 0.0063 & 0.0884
\end{array}\right) \\
& c_{3 \rightarrow 3}^{i j}=\left(\begin{array}{lll}
0.2959 & 0.1578 & 0.0277 \\
0.1062 & 0.2297 & 0.2059 \\
0.0010 & 0.0108 & 0.1671
\end{array}\right) \varepsilon_{2 j \mathrm{i}}^{i j}=\left(\begin{array}{llll}
0.0443 & 0.0209 & 0.0033 \\
0.0115 & 0.0321 & 0.0252 \\
0.0000 & 0.0011 & 0.0210
\end{array}\right)
\end{aligned}
$$

Veto:

E:

$$
\begin{aligned}
& c_{2 \rightarrow 2}^{i j}=\left(\begin{array}{lll}
0.4151 & 0.1836 & 0.0191 \\
0.1079 & 0.3129 & 0.2234 \\
0.0004 & 0.0170 & 0.2660
\end{array}\right) \varepsilon_{3 \rightarrow 2}^{i j}=\left(\begin{array}{llll}
0.1735 & 0.0920 & 0.0114 \\
0.0490 & 0.1201 & 0.0966 \\
0.0002 & 0.0063 & 0.0624
\end{array}\right) \\
& \varepsilon_{3 \rightarrow 3}^{i j}=\left(\begin{array}{lll}
0.2053 & 0.1086 & 0.0165 \\
0.0668 & 0.1468 & 0.1238 \\
0.0004 & 0.0082 & 0.0911
\end{array}\right) \varepsilon_{2 \rightarrow 1}^{i j}=\left(\begin{array}{lll}
0.0301 & 0.0175 & 0.0014 \\
0.0099 & 0.0230 & 0.0179 \\
0.0000 & 0.0009 & 0.0152
\end{array}\right)
\end{aligned}
$$

50:

$$
\begin{aligned}
& e_{I-2}^{j}=\left(\begin{array}{lll}
0.4243 & 0.1044 & 0.0192 \\
0.1111 & 0.3181 & 0.2275 \\
0.0004 & 0.0171 & 0.2746
\end{array}\right) \varepsilon_{3 \rightarrow 2}^{i j}=\left(\begin{array}{lll}
0.1772 & 0.0869 & 0.0126 \\
0.0483 & 0.1277 & 0.1051 \\
0.0003 & 0.0062 & 0.0916
\end{array}\right) \\
& \epsilon_{2 \rightarrow}^{i j}=\left(\begin{array}{lll}
0.2063 & 0.1122 & 0.0150 \\
0.0672 & 0.1505 & 0.1229 \\
0.0003 & 0.0092 & 0.0957
\end{array}\right) \epsilon_{2-3}^{i j}=\left(\begin{array}{lll}
0.0224 & 0.0127 & 0.0009 \\
0.0070 & 0.0169 & 0.0120 \\
0.0000 & 0.0007 & 0.0085
\end{array}\right)
\end{aligned}
$$

P:

$$
\begin{aligned}
& \epsilon_{2 \rightarrow 2}^{j j}=\left(\begin{array}{lll}
0.4258 & 0.1958 & 0.0181 \\
0.1085 & 0.3229 & 0.2266 \\
0.0004 & 0.0173 & 0.2815
\end{array}\right) \epsilon_{3-2}^{j j}=\left(\begin{array}{lll}
0.1637 & 0.0793 & 0.0118 \\
0.0470 & 0.1170 & 0.0975 \\
0.0001 & 0.0063 & 0.0987
\end{array}\right) \\
& \varepsilon_{\text {L }_{3}}^{i j}=\left(\begin{array}{lll}
0.2312 & 0.1203 & 0.0147 \\
0.0717 & 0.1661 & 0.1338 \\
0.0004 & 0.0093 & 0.1083
\end{array}\right) \varepsilon_{2 \rightarrow 3}^{i j}=\left(\begin{array}{lll}
0.0238 & 0.0121 & 0.0017 \\
0.0079 & 0.0174 & 0.0124 \\
0.0000 & 0.0007 & 0.0009
\end{array}\right)
\end{aligned}
$$


PO:

$$
\begin{aligned}
& \varepsilon_{2 \rightarrow 2}^{11}=\left(\begin{array}{lll}
0.4247 & 0.1940 & 0.0181 \\
0.1075 & 0.3230 & 0.2256 \\
0.0004 & 0.0169 & 0.2821
\end{array}\right) \quad \varepsilon_{3-2}^{j j}=\left(\begin{array}{lll}
0.1564 & 0.0749 & 0.0103 \\
0.0439 & 0.1106 & 0.0923 \\
0.0001 & 0.0066 & 0.0857
\end{array}\right) \\
& \varepsilon_{3-3}^{j}=\left(\begin{array}{lll}
0.2383 & 0.1257 & 0.0157 \\
0.0751 & 0.1713 & 0.1391 \\
0.0004 & 0.0093 & 0.1122
\end{array}\right) \varepsilon_{2 \rightarrow 3}^{j j}=\left(\begin{array}{lll}
0.0263 & 0.0134 & 0.0015 \\
0.0066 & 0.0194 & 0.0134 \\
0.0000 & j .0009 & 0.0102
\end{array}\right)
\end{aligned}
$$

D:

$$
\begin{aligned}
& \varepsilon_{j-2}^{j j}=\left(\begin{array}{lll}
0.4283 & 0.1940 & 0.0184 \\
0.1101 & 0.3244 & 0.2285 \\
0.0004 & 0.0381 & 0.2779
\end{array}\right) \varepsilon_{3-2}^{i j}=\left(\begin{array}{lll}
0.1591 & 0.0840 & 0.0118 \\
0.0466 & 0.1167 & 0.0902 \\
0.0001 & 0.0055 & 0.0736
\end{array}\right) \\
& \varepsilon_{3-3}^{i j}=\left(\begin{array}{lll}
0.2214 & 0.1206 & 0.0163 \\
0.0725 & 0.1535 & 0.1337 \\
0.0004 & 0.0089 & 0.0983
\end{array}\right) \varepsilon_{3-3}^{i j}=\left(\begin{array}{lll}
0.0214 & 0.0106 & 0.0016 \\
0.0064 & 0.0153 & 0.0124 \\
0.0000 & 0.0002 & 0.0092
\end{array}\right)
\end{aligned}
$$

G:

$$
\begin{aligned}
& \epsilon_{2-2}^{i j}=\left(\begin{array}{lll}
0.4083 & 0.1821 & 0.0160 \\
0.1018 & 0.3189 & 0.2156 \\
0.0003 & 0.0179 & 0.2790
\end{array}\right) \epsilon_{3 \rightarrow 2}^{i j}=\left(\begin{array}{lll}
0.1353 & 0.0646 & 0.0077 \\
0.0391 & 0.0956 & 0.0704 \\
0.0001 & 0.0058 & 0.0760
\end{array}\right) \\
& \epsilon_{3-3}^{i j}=\left(\begin{array}{lll}
0.2674 & 0.1388 & 0.0182 \\
0.0830 & 0.1936 & 0.1590 \\
0.0004 & 0.0096 & 0.1301
\end{array}\right) c_{2-3}^{i j}=\left(\begin{array}{lll}
0.0489 & 0.0239 & 0.0030 \\
0.0111 & 0.0318 & 0.0267 \\
0.0001 & 0.0010 & 0.0210
\end{array}\right)
\end{aligned}
$$

Nonveto:

E;

$$
\begin{aligned}
& \varepsilon_{2 \rightarrow 2}^{i j}=\left(\begin{array}{lll}
0.4101 & 0.1915 & 0.0211 \\
0.1256 & 0.32 \pi & 0.2349 \\
0.0006 & 0.0190 & 0.2838
\end{array}\right) \epsilon_{2 \rightarrow 2}^{i j}=\left(\begin{array}{lll}
0.1994 & 0.1033 & 0.0164 \\
0.0605 & 0.1544 & 0.1334 \\
0.0003 & 0.0071 & 0.1121
\end{array}\right) \\
& \epsilon_{3 \rightarrow 3}^{i j}=\left(\begin{array}{lll}
0.2582 & 0.1355 & 0.0306 \\
0.1053 & 0.2113 & 0.1831 \\
0.0019 & 0.0133 & 0.1502
\end{array}\right) \varepsilon_{3-3}^{i j}=\left(\begin{array}{lll}
0.0035 & 0.0100 & 0.0015 \\
0.0120 & 0.0290 & 0.0210 \\
0.0001 & 0.0008 & 0.0181
\end{array}\right)
\end{aligned}
$$


E0:

$$
\begin{aligned}
& \epsilon_{2 \rightarrow 2}^{i j}=\left(\begin{array}{lll}
0.4188 & 0.1975 & 0.0212 \\
0.1285 & 0.3360 & 0.2420 \\
0.0007 & 0.0189 & 0.2907
\end{array}\right) \varepsilon_{2 \rightarrow 2}^{i j}=\left(\begin{array}{lll}
0.1973 & 0.1018 & 0.0156 \\
0.0659 & 0.1528 & 0.1248 \\
0.0008 & 0.0090 & 0.1248
\end{array}\right) \\
& \varepsilon_{3 \rightarrow 3}^{i j}=\left(\begin{array}{lll}
0.2605 & 0.1336 & 0.0268 \\
0.0984 & 0.2162 & 0.1799 \\
0.0011 & 0.0114 & 0.1416
\end{array}\right) \varepsilon_{3 \rightarrow 3}^{i j}=\left(\begin{array}{lll}
0.0238 & 0.0127 & 0.0018 \\
0.0080 & 0.0187 & 0.0147 \\
0.0001 & 0.0009 & 0.0125
\end{array}\right)
\end{aligned}
$$

P:

$$
\begin{aligned}
& \epsilon_{2 \rightarrow 2}^{i j}=\left(\begin{array}{lll}
0.4198 & 0.1934 & 0.0192 \\
0.1258 & 0.3348 & 0.2353 \\
0.0006 & 0.0191 & 0.2949
\end{array}\right) \epsilon_{2-2}^{j j}=\left(\begin{array}{lll}
0.1777 & 0.0006 & 0.0162 \\
0.0599 & 0.1424 & 0.1137 \\
0.0005 & 0.0073 & 0.1125
\end{array}\right) \\
& \epsilon_{3 \rightarrow 3}^{i j}=\left(\begin{array}{lll}
0.2753 & 0.1412 & 0.0244 \\
0.1020 & 0.2280 & 0.1899 \\
0.0012 & 0.0127 & 0.1572
\end{array}\right) \epsilon_{1-3}^{i j}=\left(\begin{array}{lll}
0.0236 & 0.0166 & 0.0019 \\
0.0082 & 0.0169 & 0.0146 \\
0.0001 & 0.0008 & 0.0117
\end{array}\right)
\end{aligned}
$$

P0:

$$
\begin{aligned}
& \varepsilon_{2 \rightarrow 2}^{i j}=\left(\begin{array}{lll}
0.4177 & 0.1920 & 0.0191 \\
0.1246 & 0.3338 & 0.2331 \\
0.0006 & 0.0189 & 0.2950
\end{array}\right) \varepsilon_{2-2}^{j i}=\left(\begin{array}{lll}
0.1705 & 0.0855 & 0.0140 \\
0.0553 & 0.1317 & 0.1069 \\
0.0003 & 0.0077 & 0.1081
\end{array}\right) \\
& \varepsilon_{3 \rightarrow 3}^{i j}=\left(\begin{array}{lll}
0.2835 & 0.1407 & 0.0259 \\
0.1061 & 0.238 & 0.1966 \\
0.0014 & 0.0123 & 0.1621
\end{array}\right) \varepsilon_{3-3}^{j j}=\left(\begin{array}{lll}
0.0249 & 0.0162 & 0.0021 \\
0.0090 & 0.0179 & 0.0154 \\
0.0001 & 0.0011 & 0.0122
\end{array}\right)
\end{aligned}
$$

D:

$$
\begin{aligned}
& \epsilon_{2 \rightarrow 2}^{i j}=\left(\begin{array}{lll}
0.4224 & 0.2016 & 0.0211 \\
0.1268 & 0.3362 & 0.2374 \\
0.0006 & 0.0191 & 0.2958
\end{array}\right) \quad \epsilon_{2-2}^{i j}=\left(\begin{array}{lll}
0.1775 & 0.0844 & 0.0131 \\
0.0590 & 0.1473 & 0.1133 \\
0.0001 & 0.0083 & 0.0956
\end{array}\right) \\
& \varepsilon_{3-3}^{i j}=\left(\begin{array}{lll}
0.2786 & 0.1446 & 0.0314 \\
0.1079 & 0.2226 & 0.1978 \\
0.0016 & 0.0131 & 0.1651
\end{array}\right) \varepsilon_{2-3}^{i j}=\left(\begin{array}{lll}
0.0208 & 0.0113 & 0.0013 \\
0.0082 & 0.0178 & 0.0175 \\
0.0003 & 0.0002 & 0.0091
\end{array}\right)
\end{aligned}
$$


G:

$$
\begin{aligned}
& \varepsilon_{2 \rightarrow 2}^{i j}=\left(\begin{array}{lll}
0.3958 & 0.1826 & 0.0160 \\
0.1171 & 0.3201 & 0.2183 \\
0.0006 & 0.0195 & 0.2867
\end{array}\right) \varepsilon_{2 \rightarrow 2}^{i j}=\left(\begin{array}{lll}
0.1412 & 0.0643 & 0.0105 \\
0.0122 & 0.1156 & 0.0619 \\
0.0001 & 0.0073 & 0.0944
\end{array}\right) \\
& \epsilon_{3-3}^{1 j_{3}}=\left(\begin{array}{lll}
0.3107 & 0.1623 & 0.0284 \\
0.1159 & 0.2516 & 0.2147 \\
0.0013 & 0.0129 & 0.1804
\end{array}\right) \quad \epsilon_{3-3}^{\psi_{-3}}=\left(\begin{array}{lll}
0.0461 & 0.0256 & 0.0029 \\
0.0129 & 0.0296 & 0.0279 \\
0.0002 & 0.0003 & 0.0222
\end{array}\right)
\end{aligned}
$$




\section{Appendix B}

\section{Coefficients for the Jet Rates}

\section{Calculations}

\begin{tabular}{|c|c|c|c|c|c|c|c|}
\hline$y_{\text {ere }}$ & $\boldsymbol{A}$ & B & $C$ & $y_{\text {out }}$ & $A$ & $\bar{B}$ & $C$ \\
\hline$E$ & & & & E0 & & & \\
\hline 0.01 & 37.24 & 668.4 & 334.8 & 0.01 & 37.24 & 84.4 & 334.8 \\
\hline 0.015 & 29.36 & G6L.0 & 187.6 & 0.015 & 29.36 & 238.9 & 187.6 \\
\hline 0.02 & 24.35 & 615.4 & 116.6 & 0.02 & 24.35 & 265.8 & 116.6 \\
\hline 0.03 & 18.10 & 515.6 & 52.4 & 0.03 & 18.10 & 289.2 & 52.4 \\
\hline 0.04 & 14.21 & 430.9 & 26.0 & 0.04 & 14.21 & 261.7 & 26.0 \\
\hline 0.05 & 11.52 & 362.7 & 13.4 & 0.05 & 11.52 & 229.8 & 13.4 \\
\hline 0.06 & 0.53 & 307.5 & 6.99 & 0.05 & 9.53 & 199.9 & 6.99 \\
\hline 0.08 & 6.75 & 224.9 & 1.77 & 0.08 & 6.75 & 149.9 & 1.77 \\
\hline 0.10 & 4.92 & 167.0 & 0.343 & 0.10 & 4.92 & 112.0 & 0.343 \\
\hline 0.13 & 3.12 & 108.2 & & 0.13 & 3. 12 & 71.9 & \\
\hline 0.16 & 1.98 & 69.9 & & 0.10 & 1.98 & 45.2 & \\
\hline 0.19 & 1.21 & 44.3 & & 0.19 & 1.21 & 27.4 & \\
\hline 0.23 & 0.57 & 22.4 & & 0,23 & 0.57 & 12.6 & \\
\hline 0.28 & 0.13 & 7.12 & & 0.28 & 0.13 & 3.17 & \\
\hline
\end{tabular}


APPENDIX B. COEFFICIENTS FOR THE JET RATES CALCULATIONS 296

\begin{tabular}{|c|c|c|c|c|c|c|c|}
\hline$V_{0}=$ & $A$ & $\boldsymbol{B}$ & $\boldsymbol{C}$ & $x_{0}$ & $A$ & $B$ & $C$ \\
\hline$P$ & & & & $\mathrm{D}$ & & & \\
\hline 0.01 & 37.24 & -145.5 & 334,8 & 0.01 & 15.71 & 154.3 & 83.98 \\
\hline 0.015 & 29.36 & 47.80 & 187.6 & 0.015 & 12.32 & 149.1 & 46.02 \\
\hline 0.02 & 24.35 & 121.6 & 116.6 & 0.02 & 10.21 & 137.1 & 28.02 \\
\hline 0.03 & 18.j0 & 161.0 & 52.4 & 0.03 & 7.61 & 113.2 & 12.10 \\
\hline 0.04 & 14.21 & 156.6 & 26,0 & 0.04 & 6.00 & 93.63 & 5.76 \\
\hline 0.05 & 11.52 & 141.1 & 13.4 & 0.05 & 4.89 & 78.13 & 2.86 \\
\hline 0.06 & 9.53 & 123.5 & 6.99 & 0.06 & 4.06 & 65.71 & 1.43 \\
\hline 0.08 & 6.75 & 91.04 & $1 . \pi$ & 0.08 & 2.90 & 47.31 & 0.349 \\
\hline 0.10 & 4.92 & 65.16 & 0.343 & 0.10 & 2.12 & 34.56 & 0.0714 \\
\hline 0.13 & 3.12 & 37.41 & & 0.13 & 1.36 & 21.76 & \\
\hline 0.16 & 1.98 & 19.42 & & 0.16 & 0.863 & 13.55 & \\
\hline 0.19 & 1.21 & 8.12 & & 0.19 & 0.530 & 8.14 & \\
\hline 0.23 & 0.57 & 0.048 & & 0.23 & 0.246 & 3.63 & \\
\hline 0.28 & 0.13 & -2.84 & & 0.28 & 0.053 & 0.658 & \\
\hline PD & & & & G & & & \\
\hline 0.01 & 37.24 & -130.19257 & 334.8 & 0.01 & 0.0 & 0.0 & 0.0 \\
\hline 0.015 & 29.36 & 73.27059 & 187.6 & 0.015 & 0.0 & D. 0 & 0.0 \\
\hline 0.02 & 24.35 & 149.52218 & 116.6 & 0.02 & 34.51 & -595.3 & 750.9 \\
\hline 0.03 & 18.10 & 188.56036 & 52.4 & 0.03 & 26.76 & -244.0 & 435.3 \\
\hline 0.04 & 14.21 & 182.77556 & 26.0 & 0.04 & 21.79 & -86.65 & 277.1 \\
\hline 0.05 & 11.52 & 166.08951 & 13.4 & 0.05 & 18.24 & -6.84 & 185.7 \\
\hline 0.08 & 9.53 & 147.45415 & 6.93 & 0.06 & 15.52 & 35.71 & 128.5 \\
\hline 0.08 & 6.75 & 113.41357 & 1.77 & 0.08 & 11.61 & 69.67 & 64.76 \\
\hline 0.10 & 4.92 & 86.26788 & 0.343 & 0.10 & 8.89 & 74.64 & 3360 \\
\hline 0.13 & 3.12 & 56.64114 & & 0.13 & 6.07 & 64.39 & 12.41 \\
\hline 0.16 & 1.98 & 36.50658 & & 0.16 & 4.14 & 48.97 & 4.21 \\
\hline 0.19 & 1.21 & 22.70143 & & 0.19 & 2.75 & 34.27 & 1.23 \\
\hline 0.23 & 0.57 & 10.71281 & & 0.23 & 1.45 & 18.47 & 0.217 \\
\hline 0.28 & 0.13 & 2.12175 & & 0.28 & 0.390 & 5.46 & \\
\hline
\end{tabular}




\section{Appendix C}

\section{The Mathematical Formulation of Unfolding $R_{3}^{j}$}

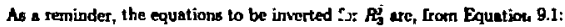

$$
\begin{aligned}
& n_{2}^{i}=\sum_{j=1}^{3}\left(\varepsilon_{(2-3)}^{j}\left(1-R_{j}^{j}\right)+\varepsilon_{(j-2)}^{i j} R_{j}^{j}\right) f^{j} N \\
& n_{j}^{j}=\sum_{j=1}^{J}\left(\varepsilon_{(j-3)}^{i j} R_{j}^{j}+\varepsilon_{(2-3)}^{j j}\left(1-R_{j}^{j}\right)\right) f^{j} N .
\end{aligned}
$$

The index $j$ refers to the prinordial quaric flavor; $i$ denota the fluvor tag which selected a particular cvent. The subscripts $n \rightarrow m$ represent "migratiop" of an event Irom having $\boldsymbol{n}$ jets at the parton level to having $m$ jets at the detector level due to axceptakiz and finite detector resolution. This equation must be solved for the $\boldsymbol{R}_{\mathrm{a}}^{j} \mathrm{~s}$, which are then used to compute $a$ :

We begin by defining the vector of inputs $\vec{Y}$ by

$$
Y=\left(\begin{array}{l}
n_{3}^{1} \\
n_{2}^{2} \\
n_{3}^{3} \\
n_{1}^{1} \\
n_{1}^{2} \\
n_{2}^{3}
\end{array}\right),
$$


the vector $\vec{A}$ as

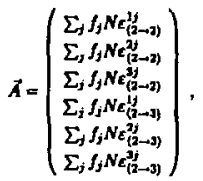

and the mator of desired quastitice $\overrightarrow{\mathbf{r}}$ by

$$
\vec{r}=\left(\begin{array}{l}
R_{3}^{1} \\
R_{2}^{2} \\
R_{3}^{2} \\
R_{3} \\
R_{3}^{2} \\
R_{5}^{2}
\end{array}\right)
$$

The matrix $B$ is given by

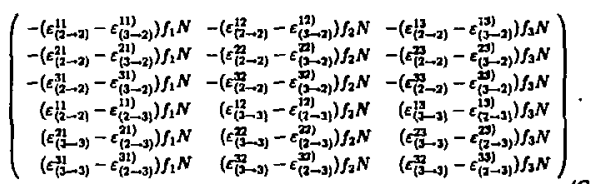

The expressions in Equation C.l can be rewritten *

$$
\bar{Y}=\vec{A}+\boldsymbol{B} \cdot \bar{r} .
$$

If we introduce the weight matrix $\boldsymbol{W}$,

$$
\boldsymbol{W}_{i j}=1 /\left(\sigma_{z} \sigma_{j}\right),
$$

then we cas write the fit $x^{2}$

$$
\chi^{2}=(\vec{Y}-\vec{A}-B \cdot \vec{r})^{T} W(\vec{Y}-\vec{A}-\boldsymbol{E} \cdot \vec{\eta})
$$


Minimizing $x^{2}$ requires

$$
\frac{\partial \chi^{2}}{\partial r}=-2 B^{T} W(\vec{Y}-\bar{A}-B \cdot \vec{r})=0 .
$$

So, $\bar{r}$ can be obtuined by

$$
\vec{r}=\left(B^{\top} W B\right)^{-1} B^{\top} W(\vec{Y}-\vec{A}) .
$$

The covarience is given by

$$
\begin{aligned}
C=\frac{1}{2} \frac{\partial^{2} x^{2}}{\partial r^{2}} & =\frac{1}{2} \frac{\partial}{\partial r}\left(-2 B^{T} W(\vec{Y}-\vec{A}-B \cdot \vec{r})\right) \\
& =B^{T} W B
\end{aligned}
$$




\section{Appendix D}

\section{Experience with Accelerator Backgrounds at the SLC}

A lerge part of the reoent impromement in SLC luminosity bes resulted frota better beam diagrostics and machine stability, and this in turn has allowed us to arrive at a wore detailed understanding of the problems of experimentel backgrounds. Part of this sucess is also due to the recent stratezy of running the demping tings with their tunes uncouplad to produce vertical/horizontal ensittance ratio of approximately 1:4. This has alluwed the achievement of a significantly amaler vertical bean size throughout the axcelerstor without much growth in the harizodul size, resulting in higher instantancous luninusity and lower backgounds[210]. Currently, upgrades to SLC and SLD are being carried out or plansed which will increnge the bean angular divergence and possibly decreme the beam pipe radius. A large groount of effort hes recently been expepded to compare the background inodels with obervations of sctual detector backgrounds in order to asess the potential ilopact of the upgredes. Here, we summarizu recent experience in understanding and controlling backgrounds from the cceeleralor which have an adverbe efiect on the SLD detector".

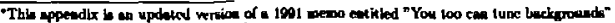

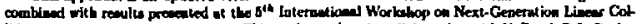

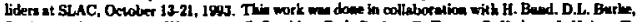

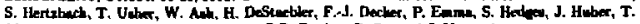

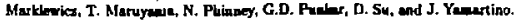




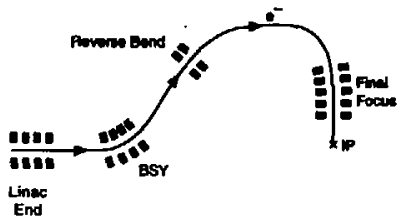

1w (Only - Are Shown)

Figure D.1: An overview of the SLC collimation kystem. A symmetric set of collimators exists in the South Arc and South Finn! Focus.

\section{D.1 The SLC Optics and Collimation System}

For completeness, this section presents a detailed introduction to the collimation systent for the SLC and its relationship to the bean orlit parameters along its length. These collinintors and orbit characteristics will be referred to throughout this pa* per. Figure D.1 shows a scheratic view of the end of the linac, the arcs, and the Final Focus, with the major groujs of collimatore labeled. Since their installation, the collimetors in sectors 29 and 30 of the 30 linac sectors have been the primary collimators for the SLC. These collinators are designed to collimate both beams simultancously, and thus are placed in alternating pairs at high-beta points for the two beams in each plane, as shown in Figure D.2. Efectively, these collimators cut the beam to a box in the $5-y$ plane, establisting the acceptance of the ben transport systetn. These are the only SLC collimators systematically deagned to define the beam phue-apace aperture. These jews are usually ued in a configuration where the primary collimation is done with the apertures in Sector 29, with the Sector 30 jaws used to clean up rone of the repopulated beam trile and to catch the spray off the upatream collimators. In the 1993 runnigg with "flat" beams of arpect ratios of approximately $\mathrm{L}: 4$ vertical to horizontal, thes collimators were typicaly at 5 beam 
sigma in the horizontal plane and 8 to 10 sigme in the vertical. Although collination to about 5 sigma is viewed a optimal[211], the tigbens of the aperture in linited by the procision and atsbility of two-bean steering in this sopnowe of the line.

Along the region of maximum borizontal diaperion at the frone of the arce nit the first aeries of momentum collinators. These allow the remowal of any low of highenergy bean tails that are the result of benn acceleration in the linac and are denoted SL-1 and SL-3. Figure D.3 shows the locations of the monblo jam at the froat of the SLC arc superimposed upon the dispersion aloug the beam orbit. Of these jers, the low-entrgy jaw is used the moot frequently, a thete is often a non-neplidible fraction of the production bunch that arives at the end of the linsc suficiently low in enerey as to be i potential problem downotrean. In addition, it bee ofter proved belpful to collimate away vertical beam tails here, w the crom-plane coupling introducad by the arc rolle will populate all undesirable corners of phane-sace by the time the beon reacher the interection point. To that ead, all of the vertical collimatore aloas the arc have been extremely useful. At about one third of the diatence dom the are, the beam curvaturn changes gign at the "Reverue Bend". As shown in Figure D.4, this provides the opportunity for another raro-dinpertion collimation point, as well 4 shother (relatively) high-dispersion point for wocondary moconatum collimation. The zero-diopersion aperture is provided by the jews SL-9. SL 10 completes the ret as the high-dispersion pair to allow removal of smalles tail that wore mined by SL 1 and 5L-3 fur upotream. Beyond SL-10, the team procoeds to the Find Focus without encountering nother set of collimators.

At the entrance to the Final Focus there lie a seriea of fixed- aperture protectina collimators. Their effect on the bean is seen primarily by the rate of Bethe-Heitler production of muons which can penet rate the SLD detector. These collimators canaot be used to remove a significant part of the bean without booding the entire detector with noive from bundreds of high energy muan (nee below.) The wot of jam labeled $\mathrm{Cl}-\mathrm{X}$ and $\mathrm{Cl}-\mathrm{Y}$ are the first movable collimators in the Final Focul. $\mathrm{Cl}-\mathrm{X}$ wen a keyhole shape, and can be moved both horizontally and vortically. Bofore the liac collimators were installed, CL-X had to function as the prianary collimator for two ontire beam-transport system. Following $\mathrm{Cl} \times \mathrm{X} / \mathrm{Y}$ are the last ueful momentum alits, 


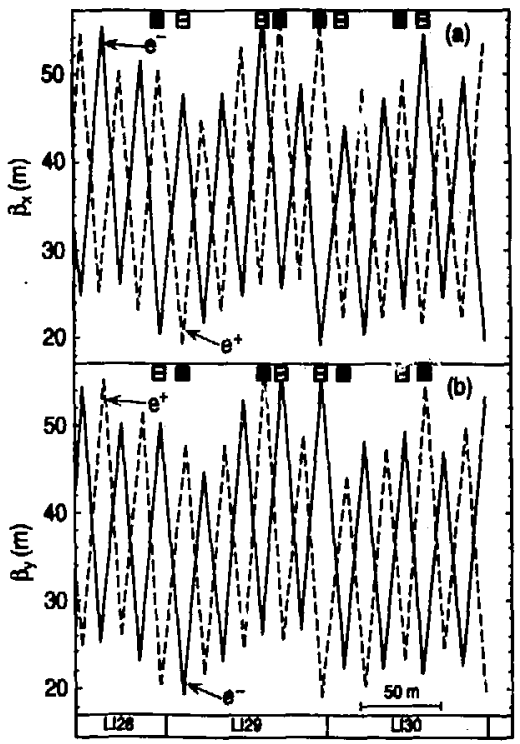

ir. Figure D.2: The Sector $\mathbf{3 0}$ collisuator layout at the end of the SLC linec. The (a) borisontal and (b) vertical $\beta$ function for electrons (positrone) ere drawn with wolid

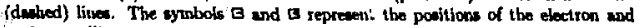
positron collimators, reapectively. . 


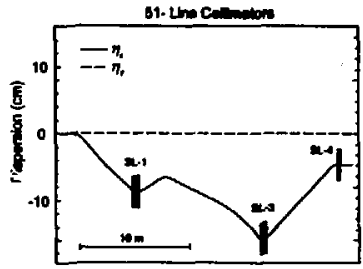

Figure D.3: The collimators in the 51-Line, at the beginning of the North Arc. The

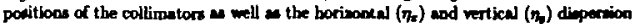
is aho shown. The double pairs of lines reprowent a complene net of vertical and horizontel jaws. SL-4 only hew vortical jaw.

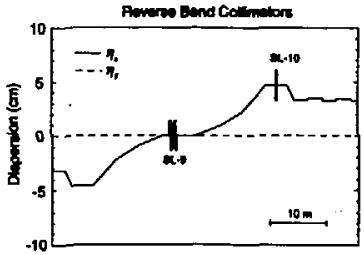

Fipure D.4: The collimators in the Revere Bend, approtimaldy one third of the

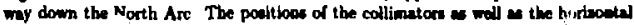

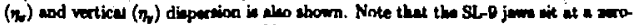
diepenton point. The two mots of SL-9 jawe are horivontal and vertical collinatorn. The AL 10 jow are borizoatal only. 


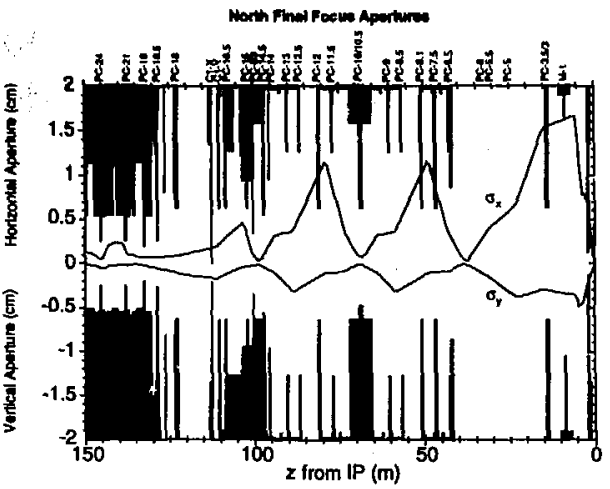

Figure D.5: The layout of the Final Focus collination system showing the poejtions of ell of the eprertures slong the beanline. Movable collimators ara shown in theis fully-inserted positions. The 100 beam cllipse in the horizontal and vertical planes is shown for optical configuration used it the 1993 SLC nunning.

PC-12, and the other movable jaws in the Final Focus, PC-10.5 and PC-10, PC-7.5, and PC-3. As discussed bolow, these collimators are used sparingly, as there is a constant neerl for compromise in how rnuch beam loss one can tolerate here versus how much beam one needs to remove to run the more semsitive parts of the SLD detector. Figure D.5 shows the 10-signa beam envelope for notninal 1993 emittances at each of the nportures in the Final Focus. 


\section{D.2 Types of Backgrounds Seen in the SLD Detector}

There are tinree major types of aceelerator-induced backgrounda vieibla in the SLD detector. A brief introduction to each is provided here. Detaled dincuesione of the backgrounds follows.

As mentioned above, muons are created by jhotoproduction from primary beam particles hitting an aperture upstrean of the interaction point (IP). As the Final Focus tunnel needs to be large enough for accen to the beasuline, there is jot sufficient material present to absorb all of these muons. Hence, the muone can travel from their production point along the beamline and can penetrate to the SLD detector.

The SLC produces copious synchrotron rediation from the focusing elements along the beamline. A masking scheme ba been implemented around the IP in an attempt to shield the sensitive portions of SLD from the intenes syuchrotron radtation produced by the final dipoles and quadrupole triplet. Particles in the tail of the beam, however, can create synchrotion radiation which atrikes the masks directly and can re-scutter into the detector, causiag unwanted noisen

In addition to these other backgrounds, also have evidence that a number of particles derived directly from the prinery beam are showering in or near the detector. These showers produce visible secondary perticles that are detexted by SLD and are a potential source of backgrounds for physies andyses.

\section{D.2.1 Muon Backgrounds}

With the initial commissioning of the SLC came the surprise that there exo numerous muons being created in the Final Focus as bean tails are scraped off on the collirators. This led to installation of magnetized iron muon toroids placed in such a manner os to deflect most of the muons away from the detector[212]. Figure D.8 shows the layout of the muon toroids relative to the collimators end detector for the north Final Focus. This approach was successful for the running of the Mark 11 detector for its years at SLC. Since SLD is a nuch larger detector, howover, it presents a much larger 


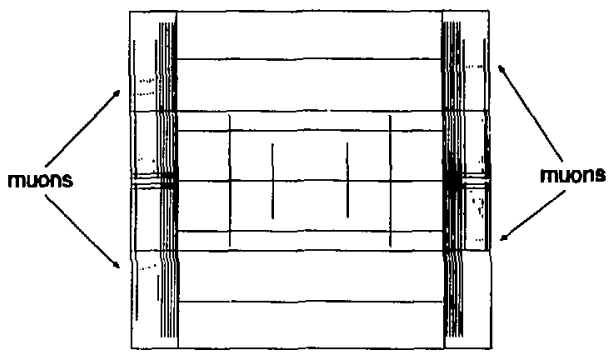

Figure D.6: A view of the SLD WIC detector, showing the pathe of muons produced by the SLC beam and traveling estentially parallel to the beam axis. The WIC endcaps have crossed strips in order to obtain the positions of the muons; only one projection can be secn; (The vertical lines arc the vertical WIC strips, which nre parallel to the plane of the page.)

target for both defected and undeflected muons. The Warm Iron Calorimeter (WIC) of SLD is a backing calorimeter and muon detector made of steel plates interspersed witb Iarocci tubes. Since most of the background muons are traveling parallel to the beam, they do not point back through the IP along tracks and thus are not confusing for muon identification. They do present a constant signal of tracks in the WIC (see Figure D.6), and thus make the creation of a trigger for low- ungle muon pairs from $Z$ decays impossible. Also, muons traversing the Liquid Argon Calorimeter (LAC) leave long continuous strips of hit towers (see Figure D.7), which can be removed but occasionally overlap with clusters of energy from particles in $\mathbf{Z}$ events, potentielly skewing the determination of event shape variables. During the 1993 run, the mean number of muons secn in the calorimeter per $\mathrm{Z}$ event was 0.32 , down from 0.7 in 1992 . This is 


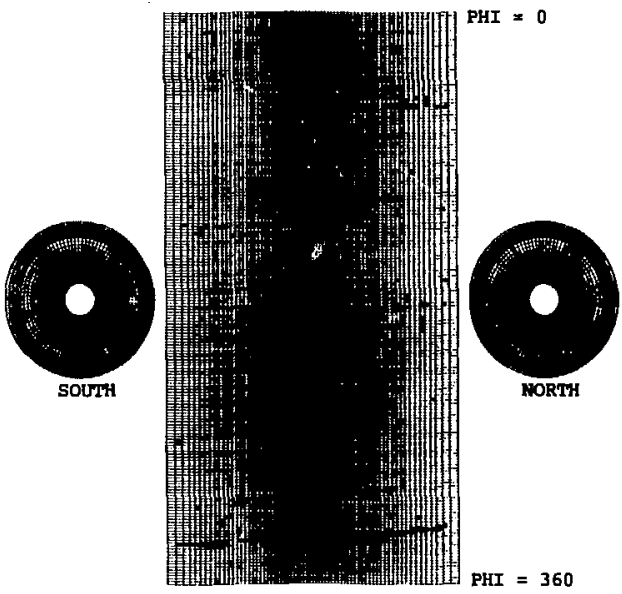

Figure D.7: A view of the SLD LAC, showing the paths of muons produced by the SLC beum and traveling esoertially parallel to the bearn axis. In the LAC barrel, the muops deposit energy in the liquid argon gape between the lead tiles; longitudinal streaks of adjacent towers sigenl the presence of these muons. The LAC bus been unrolied into a plane for this perspective. 


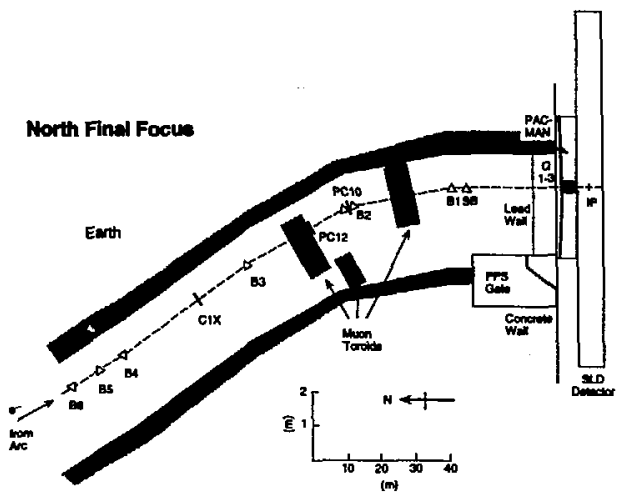

Figure D.8: The layout of the Final Focus collimation system showiag the positions of the collimators, several optical elements, and the muon-spoiler toroids. Note the difference in vertical and horizontal scales for this drawing, and the large size of the SLD detector relative to the tunnel :yert ure.

a relatively stnall perturbation on a culorimetric analysis and thus does not degrade the performance of the calorimeter by that much. The number of muon tracks in the WTC endcaps is substantially larger, causing approxinately $50 \%$ more tracks to be called muons by the muon identification system; since muons pass along the barrel axis, the WIC barrel is virtually unaffected. Infrequently, a muon passes through the Central Dritt Chamber (CDC) parallel to the sense wires, depositing hundreds of hits in a small number of jet cells. Since these hits are localized to a relntively small area of the tracking volume, they do not significantly effect the tracking efficiency. 
Studies of the locations and strengths of the muon toroids[213] were underteren to ascertain whether or not the optimum position calculated in Ref. [212] for running the Mark II detector was also the optimum for SLD. Posibilitie for some improvement over the current placement and strengths of the toroids was seen, but these heve yet to be subjected to an experimental trial.

\section{D.2.2 Synchrotron Radiation Backgrounds}

In the difficult environment of a linear collider, it is necessary to have as deteiled as possible an understending of the potential for backgrounds due to synchrotron radiation (SR). Since there is no opportunity to use scrapers or otber such devices to reduce beam tails on multiple turns of the beam around the machine, the interaction region must he designed to accommodate the additional, wider-diver gence SR from the beam tails. This makes simulations of the sources and scattering of SR photons and their interaction with the detector escential to minimize the advarpe efocts of beam tails on detector performance. Indeed, modeling of the syachrotron radintion from the final triplet followed by EGS4[214] simulation of scattering in the detector has illuatrated the expected inportance of collimation and the sensitivity to beam tails.

The problem is made more severe, however, by the constant effort to minirize the beam spot sizes at the interaction point in order to maximize the luminasity. In general, the linear spot size $\sigma^{*}$ at the IP is given by $\sigma^{*}=\epsilon / \theta^{*}$, where $\epsilon$ is the beam emittance, and $\theta^{*}$ is the angular divergence of the beam core. A larger angular divergence implies a larger beam sine in the final quadrupoles that focus the beam, which generates much more synchrotron radiation from any beam tails that are preaent. At some point, the luminosity cannot be made larger without making the detector useless. As an introduction, Figure D.9 shows the SLD IP making on a distorted scale; the overall distance along the beamline is $3 \mathrm{~m}$, and the dianeter of the M2 aperture is $27 \mathrm{~mm}$. Sensitive detector elements are shown in gray. They include the Central Drift Chamber (CDC), the CCD-pixel Vertex Detector (VXD), the Medium Angle Silicon Calorimeter (MASiC), and the silicon calorimeter Luminoaity Monitor 

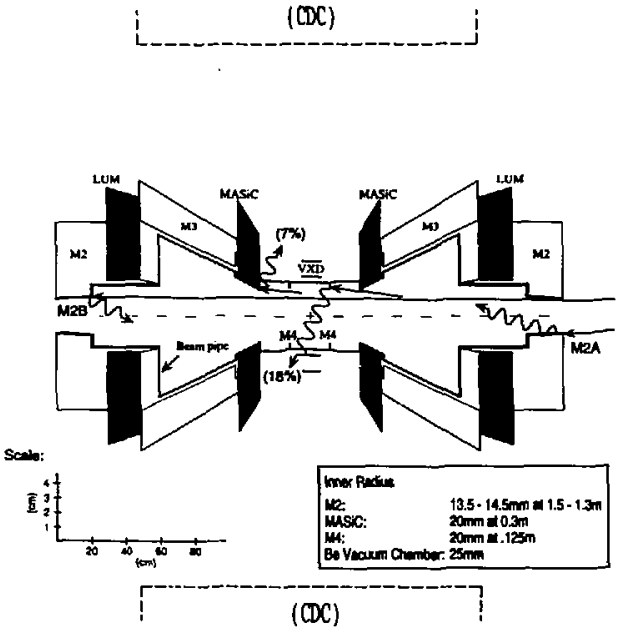

Figure D.9: A diagratn of the masking around the SLC IP disigned to shield the SLD detector comjonents from the primary synchrotron radiation produced in the focussing elements upstream. Also shown are the paths synchrotron photons must take to be scattered into the active detector elements. Primary synchrotron radiation background (which is never allowed to strike the detector) is shown in solid lines. Secondary and tertiary beckgound is in wavy lines. 
(LUM). Each detector bes a nlightly different response to SR backgrounds.

In considering the impect on physica analyes, the two moot senoitive systems are the ones leed in tracking, the CDC and the VXD. Excesive numbers of synchrotron photons in eitber detector produce randomly scattered photoelectric or compton electrons, which leave large local depositions of energy. This can result in very large currents on the CDC bigh-voltage grid, potentially damaging the chamber or increasing the rate of aging of the wires. On a sualler scale, these low energy dectrons leave huge amounts of charge in an aren equivalent to one CDC sense wire, anturating the digitization ADCs that are necessary to get pulse-shape information. This readers part of the drift length of the drift chamber cell useless for trucking, as the real track hits are obocured by the huge pulse from the secondary electron. Some capacitive coupling betwaen adjucent cells on the CDC also leads to croestalk from this lerge puleo on other nearby channels, cresting large błotches of hit cells in the CDC. From timing and charge information, this crosstalk is easily recognizuble and can be removed in soltware offilne; it does, however, wfect the operation of the tracking trieger. Drift chember doise is always quantified in terms of "oceupency", which is defined as the percent of wires (out of 5120) with hits in a given bewm croesing. Sufficiently lesse occupancy, thea, can result in a significant degradation in the detector's ahility to find and fit tracks.

In the vertex detector, these local depositions of energy translate into spurious clusters of pixels which could be linked onto the tracks projected laward trom the CDC, potentially resulting in mismeasured track parameters. Since the VXD is septially a two-layer device, the track linking is not suficiently protected from linking two beckgtound hits to an otherwise well-measured track. Thbs bas been identified as one of the largest sources of efficiency loss for linking tracks to tho VXD hits; the tbree-layer VXD3|215] will be considerably less vulnerable to this problem.

\section{Syachrotron Radiation Buckground Simulations}

It is useful to define muximum divergence angle, $\theta^{\text {wax }}$, which is obtained by multiplying the beam core divergence, $\theta^{*}$, by the aperture of the collimation system in bearn sigmas. Typlcally, $\theta^{N A X}$ is about 5 to 8 times $\theta^{*}$. This repreasnts the maximum 
passible excursion of particle traveling through the final lenses.

If the total angular spread (including tails) of the beam at the IP, $\theta^{\text {4Ax }}$, is limited by collimation to a small enough range, the only quadrupole synchrotron radjation (QSR) incident on the detector masks atrikes the interior of the downbeam M/2 mask (MI2B) after passing the IP. With the current masking, a photon frorn this mask cannot reach the beam pipe vithout additional scattering, and the probability that a pboton incident on this mask results in a photon in the CDC is ratber small $\left(6 \times 10^{-7}\right.$,

If $\theta^{\text {4ax }}$ is increased somewhat, there will also be photons incident on the upbeam M2 mask (M2A). Photons originating in this mask also must scatter again in order to resch the bearn pipe, but thoy are typically more energetic than thoee from the downbeam mask, and have a larger probability of resulting in a photon in the CDC.

If $\theta^{\mathbf{N A x}}$ is suliciencly largc, photons passing through tbe 27 mm diameter aperture in M2A can bit the annular M4 mask inside the berylium beam pipe, or the face of the Medium Angle Silicon Calorimeter (MASiC). Photons striking these surfaces directly have probabilities of $18 \%$ and $7 \%$ respectively of producing a photon in the CDC. Because the flux of photons passing through the M2A aperture can vary rapidly with radius, this situation is likely to scult in high and unstable CDC backgrousd occupancy and must be avoided.

The SR and EGS4 models can be combined with a simulation of interactions in the CDC to obtain an estimaled CDC occupancy for a given beam profile and opaque zollimators. We concentrate on the effects of bean tails or halo because a Geussian beam with nominal $\theta^{*}$ results in negligible $\mathrm{CDC}$ occupancy. In order to understand the geometry and the sensitivity of the QSR background to various parts of benm phase space it has been useful to consider a distribution of electrons uniform in $\theta_{x}^{*}$ and $\theta_{y}^{*}$, and to set a reasonable scale we assume this uniform distribution constitute $1 \%$ of the beam at the IP. In the alsence of a measurement of the shepe or magnitude of benm tails we use the preceding assumptions as a model of a 'flat tail'.

The genetal behavior of the ealculated CDC occupancy due to QSR is illustrated in Fig. D.10 where we show the occupancy for this model as a function of $\theta^{\mathbf{N a x}}$. This particular graph is for the case of round beams with $\theta_{x}^{\mathbf{N a x}}=\theta_{y}^{\mathbf{N A x}}$. The occupancy due to scattering from the downbeam mask (M2B) is relatively small and 


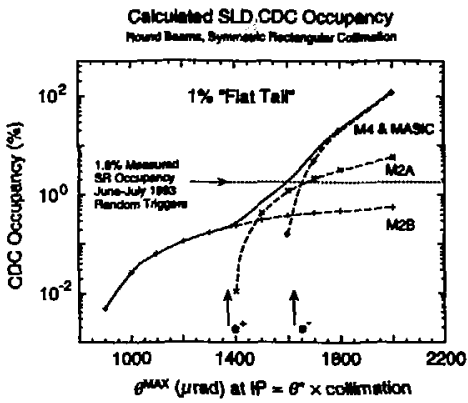

Figure D.10: Measured and calculated CDC occupancies. The solid curve is the calculated occupancy in the central drift chamber due to syacturotron radistion from the quadrupoles as a function of the maximum beam divergence angle at the interaction point, assuming ruund beams and $1 \%$ of the beam spread out in a vary wide teil. The dashed curves show the contributions from each mask. Typical operating points are indicated by the arruws below the graph, which show approximate values for each beam in Juse 1993. Also shown is a measured occupancy due to synchrotron radiation, determined from a sample of random trigger events recorded in June and July 1993.

independent of $\theta^{\kappa \wedge x}$ in the rasge of interest, and occupancy dise to QSR incident on the upbeam mask (M2A) is significant for $\theta^{\text {mAx }}$ larger than $1400 \mu \mathrm{red}$. Beyond $1600 \mu \mathrm{rad}$ scattering from MA and the MIASiC is significant, and quickly dominates as $\theta^{\text {HAX }}$ increases further. 


\section{D.3 Spurious Track Trigger Background}

In orier to collect events from $\mu$ - ard t-peir decnys that do not depoait lars: amounts of exergy in the calorimeter, SLD has implemented an event trigger beed solely on charged tracks in the CDC. For an event to pass the trigger requirements, there must be at least two charged tracis separated by more then $150^{\circ}$. Since the trigger's initial commissiouing, it hes been clear that large numbers of charged particles are being produced in beam crossings that contain no other electromeak physics proceses. Occasionally, the rate of hard charged particle production exceeds $0.1 \mathrm{~Hz}$, and the trigger has to be prescalod or rate-limiterl to avoid excesive dend time in the detector, cauring a potential los of events fron $\mathrm{ZO}$ decays. We have undertaben an extensive study of these events to understand their source, and sorpe of the results are presented below.

The charged particles in these events bave a transverse momentum spectrum with an average of $400 \mathrm{MoV} / \mathrm{c}$, where the mean aumentum for the fastest particle is about $1 \mathrm{GeV} / \mathrm{c}$. The average charged multiplicity of these events is approximately 5 tracks per evert. The particles with enough transverse moment um to fire the tracking trigger have been shown through a $\mathrm{dE} / \mathrm{dx}$ analysis to be predominantly protons, and the ratio of positive to negative particles is 7:1. Nll of thene attributes suggeat that it is impossible that these tracks are due to interactions from synchrotron radiation, as the SR spectrum typically has very few photons above $5 \mathrm{MeV}$. The presence of large numbers of protons also implies energetic interactions with the material sursounding the detertor. In eddition, since the SR spectrum falls of quite rapidly with energy, there would be a huge ocrupancy in the CDC due to the lower energy photons which would also scatter into the detector. The scattering process does harden the SR spect rum somewhat, but not nearly enough to produce only tho high-energy particles secn in these events.

Figure D.I1 shows the position in 2 relative to the nominal IP of reconstructed chrogid track vertices from events which fired the tracking trigger. Approximately Gofrom the two masks M4 located $12 \mathrm{~cm}$ to either side of the interaction point. Single-beam studies have shown that the dip angles of the tracks are inclined along 


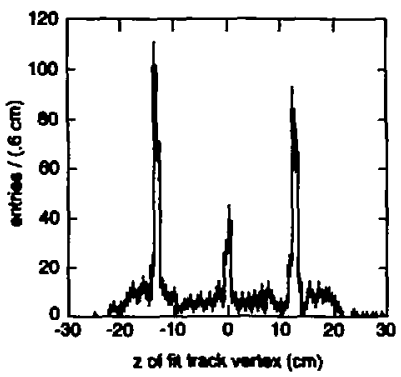

Figure D.11: The primary vertex poxitions along the beam axis in low-multiplicity tracking-trieser evente. Some number of twa-photon events or low-multiplifity $Z$ events which conse from the SLC IP are inciuded in the sample, giving the peak at $z=0$. Hownever, most of the events come from interactions in the M4 rmenks which are located at :t $12 \mathrm{cmu}$ from the IP.

the direction of the beam that produced them, but no strong correlation is observed between the track direction and whether they bit upstream or downstrearn of the IP. An andysis of vertices of the tracks in the transverse plane clearly shows that mout of the tracks originate in the top and bottom of the beam pipe/maks, whown in Figure D.12.

There is also often a correlation between the presence of tracks in the CDC and deposits of lerge elumps of isolated energy in the luminosity monitor. These also occur mostly in the top and botton of the luminosity monitor, but do not have a strong correlation to the location of the irack vertices found in the same event. The isolated ehowers in the luminosity monitor have a different longitudinal profile then ieolated electrons from Bhabhe scattering, as the frection of eacras deponited in the fiset of the two layese is wuch lerger in the caes of the background events, implying 


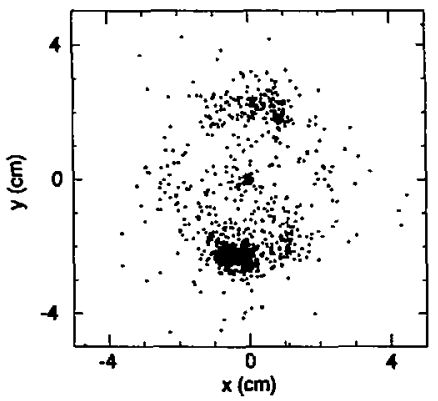

Figure D.12: The $x-y$ primary vertex positions in low-multiplicity tracking-trigget events. Some number of two-photon events or low-multiplicity $Z^{0}$ events which come from the SLC IP are included in the sample, but most of the events come from intersctions at the top and bottom of the beampipe/masks.

that the shower has already started before the particle hits the luminosity monitor. This is supported by numerous tracking trigger events where the inmertmost layer of the MASiC has a shower which correlates exactly in azimuthal position to the luminosity monitor shower behind it. Gcometrically, a particle entering from outside the detector hes to hit she MASiC first to deposit emergy into the LUM, ouggesting that these energetic particles do not originate at the IP. In addition, there are often multiple high-energy isolated showers in events where the total energy deposited in the luminosity monitor is hundreds of GeV. 


\section{D.4 SR Background Measurements}

SLD routinely wites to tape a sumple of ovente obteined by trigsering on rasdom beam cromings. The andysis of a oet of random trigsen from June and July 1903 resulted in a value of $2.3 \%$ CDC occupancy due to synchrot ron radiation. Suberactine $0.5 \%$ for radiation from the leat SLC bepd mapnet gives $1.8 \%$ due to the quadrupoles, which is indicated on Figure D.10. Typicel SLC operating conditions for a 24-bour period in mid-Jutne 1993 are os follows:

$$
\begin{aligned}
& \text { Flectrons: } \theta_{r}^{\mathrm{MAX}}=4.5 \times 350 \mu \mathrm{rad}=1575 \mu \mathrm{red} \\
& \theta^{\mu / \alpha x}=9.5 \times 175 \mu \mathrm{rod}=1663 \mu \mathrm{rad} \\
& \text { Positrons: } \theta_{2}^{\mu a x}=4.5 \times 350 \mu \mathrm{red}=1575 \mu \mathrm{rad} \\
& 0)^{\text {vax }}=8.8 \times 150 \mu \mathrm{rad}=1320 \mu \mathrm{rad}
\end{aligned}
$$

Figure D.10 indicates these operating conditions. Note that the coilimation mons to result in what are efectively "round" bean tails. This is, as mentioned above, becaure the bearn positions at the collimator jaws at the end of the linac is suficiciently unetable as to require a slightly larger aperture to limit the posibility of large beam looves and the even larger backgrounds that this causer. In this case, the electron beam would be expected to produce incost of the CDC occupancy. The occupancy predicted by the simple model agres surprisingly well with the meatured value. However, the $0^{\text {wax }}$ values above must be taken as very approcimate, as the collimation in the $x-y$ plane at the end of the linac results in a rectangular cut in $x-y$ position spece. Particies up to $50 \%$ farther any from the core of the beam can thus remain in the beam if they sit in the epproprinte location in space. SLC bistory plots of beam size and angular divergence show significant time veriation, but it is common to operate in this situation, with one benr contributing nepligihle occupancy, and the of ber on the edge, contributing several percent background CDC sccupancy.

Esen though the "effective collination" calculated above for runs during 1993 gives equel $\theta^{\text {uax }}$ valtes for both the $I$ and $y$ planes, the smaller beam emittance in the vertical hes had a significant effect on the severity of beckgrounds experienced by SLD. Comparing good running conditions from the 1992 run with rune having 
sirdler $\theta^{\text {Hax }}$ values from 1993, we have ceen a reduction of opproximately a factor of 3 in the average CDC occupency due to SR in the 1993 data telative to 1992. This suggests tbat our assumption of a $1 \%$ Hat tail as the source of SR backgrounds to be an overestinnte of the tail population, or that the comprasition of the tails primarily responsible has changed as beam conditions have improved. In addition, due to the smallex angulor divergence in 1993, the sertical beam size in the Final Foeus is also smaller, which may reduce the amount of bean tail generated there. The optical properties of the final quadrupole triplet also may contribute to this reduction in beckground. From Figure D.5, one can see that the beam has a large vertical $\boldsymbol{\beta}$ function closer to the interaction point than in the borizontal plane, and that it decreases rapidly to the IP. This violent focusing is expected to generate a larger amount of synchrotron radiation. This problem would be ameliorated if the beam size in the vertical plane were substantially smallex, as there would be fewer particles that would Ieel the stronger region of magnetic field within the quad.

Late in the 1993 run two shifts were dewoted to beckground studies with single beams. Anslysis of this dats is continuing, but some preliminary conclusions can be made. The collimation was not optimized for the positron beam, and beceuse of time constraints most of the data were taken with the electson bearn.

SLD data were recorded at four values of $\theta^{*}$ for the electron beam: anal] $\theta^{*}$, the $\theta^{*}$ at the end of the $1993 \mathrm{rum}, \theta^{*}$ expected in 1994, and a larger value. For the seasons mentioned above, positson data was oblained only for the first two vives. The CDC occupanty due to synchrotron radiation, determined from random bean crossiog data, is shown for electrons and positrons in Figure D.13 as a function of both $\theta_{y}$ and $\theta_{y}^{\mu \wedge x}$. In the first case the occupancies uppear to diffur significantly, but when plotted as a function of $\theta_{y}^{N A x}$ the electron and positron occupancies are quite similar, illustrating the importance of collimation on backgrounds. Figure $D .13$ also shows the occupancy calculated as for Figure D.10, except that the measuted values of $\theta_{r}^{*}, \theta_{v}$ and the actual $\mathrm{X}$ and $\mathrm{Y}$ collimator settings bave been used. The agreement between the date and this simple model is roasonable, but not dramatic.

The data taken at the small $\theta^{\circ}$ point has been used to measure the SR due only to the and bend magnet before the IP, as this is an important buseline number in the 

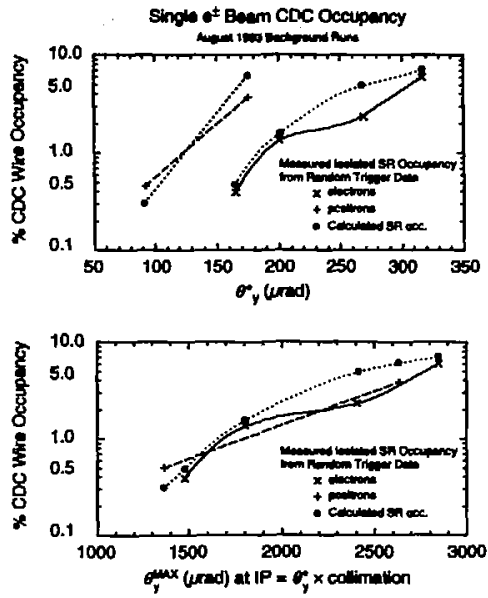

Figure D.13: The solid and deshed curves aro measured CDC occupancies, using randon triggers in dedicated beckground runs, for single electron and positron bearus respectively. The dotted curve is the calculated CDC occupancy due to synchrotron radiation, assuming $1 \%$ of the beam spread out in a very wide tail, and using treasured $X$ and $Y$ values for $\theta^{\circ}$ and collimation. Otherwise the calculation is identical to that used in Figure D.10. In the upper graph the curves are plotted as a function of the mearured. In the lower graph they are plotted as a function of $\theta_{y}^{w a x}$, the product of $\theta_{;}^{*}$ and the collimation in units of the beam $\sigma_{\text {, at }}$ the collimator. The similarity of the meanured occupancy curves in the lower gaph liltustrates the importance of collimation in determining backgrounds. 
calculations of the ocupancies due to scattering off the masks, mentioned above. At this stadl angular divergence, the SR frotn the final quadrupoles is expected to be small, slowing ws to observe the dipoie SR direetly. The CDC occupancy due to synchrotron radiation from the last SLC bend magnet bas been calculated is $0.25 \%$, and the preliminary measured value for a small angular divergence (235 $\times 164 \mu \mathrm{rad})$ is between 0.25 and $0.50 \%$. This is a partid measure of the ability of the models to predict CDC oceupancy due to SR.

When the angular divergence of the electron beam was varied, the vertex detector vecupancy (\# of clusters per CCD) and chustet size (\# of pixels per cluster) did not vary significantly between current conditions and those expected after the upgrades. The obecrved increased oceupancy in the CDC and vertex detector was got large enough to gencrate encerns about bechgounds in the 1994 rum.

\section{D.5 Tracking Trigger Backgrounds Studies}

In on at tempt to understand more about the tracking trigger background, we included in the background studies at the end of the 1993 run single- beam running with reduced and nesr-zero feld in the SLD solenoid. At $10 \%$ of the nominal $6 \mathrm{~T}$ fiedd, the events look very similar to those seen in the original Mark II vortex drift chamber as shown in Ref. [211]. An event picture of the SLD CDC is shown in Figure D.14. This is a restom triggered event from this run. Any track that passes through all layers of the CDC has a momentum of at least $20 \mathrm{MeV}$ at this field and there are typically many of these tracks per event. Virtually all events bave some tracks with an energy greater than $20 \mathrm{MeV}$. A study of the CDC occuparcy as a function of radial wire layer while the B-field is varied has shown that the majority of these tracks probably come from photon conversions within the noterial between the IP and the drift chamber. Also, the $z$ of the track-beam pine intersection does not siow the dramatic, peaked structure seen in Figure D.11. The overall CDC occupancy during this runing was large but stable, implying statkstically that the number of particles causing the underlying event must be large enough not to fluctuate to zero very often. 


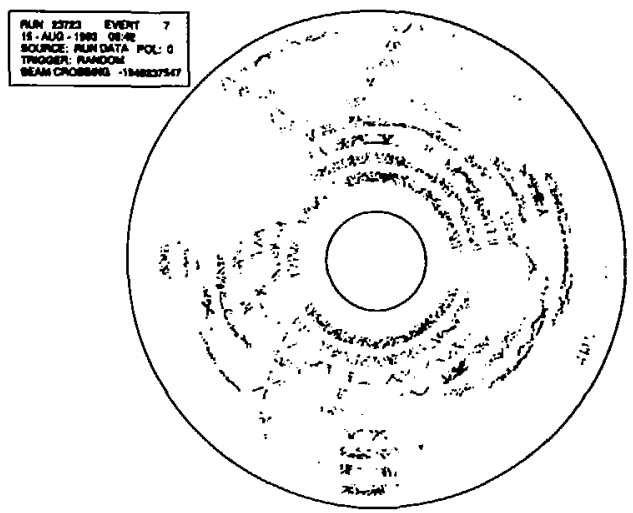

Figure D.14: An event picture showing the CDC for a random trigger during running with the electron beam only at $10 \%$ of nominal solenoid field. Tracke that traverse the entire one-meter radius have a transverse moment uin of greater ther $20 \mathrm{MeV} / \mathrm{c}$.

The above evidence leads us to the conclusion that the tracking trigger events are caused by multiple off-exis, off-energy primary bean particies showering inside the detector as sbown in Figure D.15. The pattern of vertical versus borizontal bits is also comparable to what is expected from a study of the tranoport of ofl-energy and of axis particles through the final focusing triplet, of of-axis, off-energy particles are much inore strongly over-focused in the vertical plane (see Figure D.5.). The sdditional evidence from single-bean tests with a dowered solenoid Beld inplies that this process occurs on almost every event, as the epectrum of perticles produced is 
for too energetic to come solely from synchrotron radiation. The mask M2, whome sperture has an inner radius of $1.35 \mathrm{~cm}$ at 1.5 meters from the IP, is the likely source of these scattcrs. We have also observed events in running single beams where the entire detector is flled with noise, including the upstrearn luminosity monitor, which implies that the shower must have originated before the beam has arrived at the IP. A showet near M2 is the only thing that could produce enough local energy to generate this signal. EGS studies are underwny to confirm that enough energy cas be generated in these showers to produce the obeerved tracks. In the Mark II studies discussed in Rer. [211], the observed backgrounds could be simulated by nssuming that between 10 and $10050 \mathrm{GeV}$ eloctrons hit a mask upetream of the final quadrupole triplet on every beam erossing. llowever, the SLD masking scheme is substantiblly different near the IP, so it is not clear if this will remain as an accepted explanation.

It remains to be seen whether or not the actual source of these off-energy, off-axis particles can le found and eliminated. The inser radius of $\mathbf{M 2}$, thougb it is at $\mathbf{1 . 3 5}$ $\mathrm{cm}$, is still more than 30 beam sigma from the core of the bean. The momentum bandwidth of the Final Focus bean trarsport system is narrower than this, which implies that these particles must be generated somewhere in the Final Focus itself. A ray-tracing study should probably be done to see if any potential sources can be identified. Interestingly enough, the rate of these events decreascd markedly when the angular divergence of the bat was lowered. This points strongly at some aperture in the Final Focus as a cause of these tails, as the beam in this case is much smaller as it travuls along the beandine, and thus will be more apt to miss the offending obstruction.

\section{D.6 Conclusions}

We have undert aken an extensive study of the background conditions at the SLC using the SLD detector in an attempt to ameliorale trigger problems and assess the affect of potential upgrades. Although the results are preliminary, good agreement is found between models of symchrotron raciation interactions and actual data taken from the detector. Many unsolved questions, cspecially as to the source of the tracking trigger 

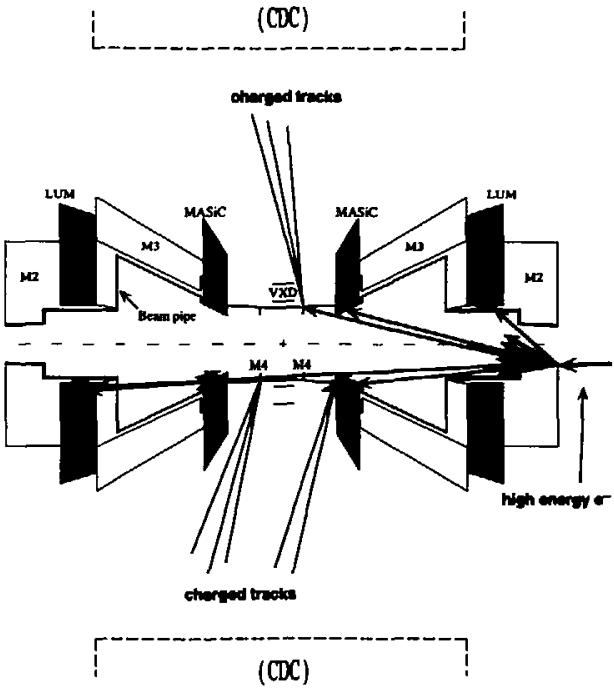

Figure D.15: Schematic showing the expected source of the track triggen. The masks are shown on the conventional diatorted scale. Pritnary, but degraded-energy, electrons strike the mask with shower debris accounting for the large energy in the low-angle calorinetry and the production of low-energy particles at large angle. 
beckground, atill remain, however, and will heve to be pursued during the uponming rus. 


\section{Appendix E}

\section{The SLD Collaboration}

K. Abe, ${ }^{(20)}$ I. Abt, ${ }^{(10)}$ C.J. Ahn, ${ }^{(26)}$ T. Abag, ${ }^{(27)}$ N.J. Alleo, ${ }^{(1)}$ W.W. Ash, ${ }^{(27) t}$ D. Aston, ${ }^{(2)}$ K.G. Baizd, (25) C. Baltay, ${ }^{(20)}$ H.R. Basd, ${ }^{(2)}$ M.B. Beralat, ${ }^{(D)}$ G. Baranko, ${ }^{(19)}$ O. Bardon, ${ }^{(16)}$ T. Barklow, ${ }^{(11)}$ A.O. Bararko, ${ }^{(14)}$ R. Ben-David, ${ }^{(D)}$ A.C. Benvenuti, ${ }^{(2)}$ T. Bienz, ${ }^{(2)}$ G.M. Bilei, ${ }^{(2)}$ D. Bicollo, (21) G. Bhayloct, (n) J.R. Bogart, ${ }^{(\pi)}$ T. Bolton, ${ }^{(11)}$ G.R. Bower, ${ }^{(2)}$ J.E. Breu, (20) M. Braidenbech, (m)

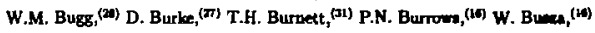
A. Calcaterra, ${ }^{(13)}$ D.O. Caldwell, ${ }^{(5)}$ D. Calkoway, ${ }^{(2 \pi)}$ B. Camanzi, ${ }^{(2)}$

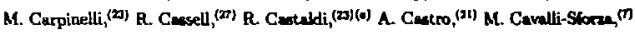
E. Church, ${ }^{(31)}$ H.O. Coinn, ${ }^{(2)}$ J.A. Coller, ${ }^{(3)}$ V. Cook, ${ }^{(3)}$ R. Cotton, ${ }^{(1)}$ R.F. Cowan, ${ }^{(16)}$ D.G. Coype, ${ }^{(j)}$ A. D'Olivejra, ${ }^{(8)}$ C.J.S. Damerel, ${ }^{(2)}$ M. Droudi, ${ }^{(\pi)}$

R. De Sangro, ${ }^{(13)}$ P. De Simone, ${ }^{(13)}$ R. Dell'Orso, ${ }^{(2)}$ M. Dima, ${ }^{(9)}$ P.Y.C. Du, ${ }^{(20)}$ R. Dubois, ${ }^{(27)}$ B.I. Eisenstein, ${ }^{(14)}$ R. Elin, ${ }^{(27)}$ D. Fulciej, ${ }^{(22)}$ C. Fon, ${ }^{(10)}$ M.J. Fero, ${ }^{(14)}$

R. Frey, ${ }^{(20)}$ K. Furuno, ${ }^{(20)}$ T. Gilmen, ${ }^{(24)}$ C. Gladding, ${ }^{(14)}$ S. Gonzeler, ${ }^{(19)}$ G.D. Hallewell, ${ }^{(\pi)}$ E.L. Hart, ${ }^{(2)}$ Y. Henegawa, ${ }^{(2 n)}$ S. Hedges, ${ }^{(1)}$ S.S. Hertshach, ${ }^{(17)}$ M.D. Hildreth, ${ }^{(2)}$ J. Huber, ${ }^{(20)}$ M.E. Hufler, ${ }^{(27)}$ E.W. Hughes, ${ }^{(27)}$ H. Hwang, ${ }^{(20)}$

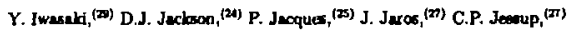

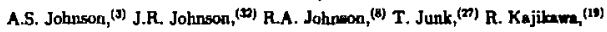
M. Kalelkar, ${ }^{(23)}$ I. Kevliner, ${ }_{t}^{(14)}$ H. Kawahera ${ }_{t}^{(27)}$ H.W. Kendall, ${ }^{(16)}$ Y. Kim, ${ }^{(20)}$ M.E. King, ${ }^{(27)}$ R. King, ${ }^{(2)}$ R.R. Kolier ${ }^{(17)}$ N.M. Krishas, ${ }^{(10)}$ R.S. Kroeger, ${ }^{(10)}$ J.F. Labs, ${ }^{; 27)}$ M. Langston, ${ }^{(20)}$ A. Lath, ${ }^{(16)}$ J.A. Lauber, ${ }^{(10)}$ D.W.G. Leith, ${ }^{(m)}$ 
X. Liu, ${ }^{(7)}$ M. Loreti, ${ }^{(2)}$ A. Lu, (9) H.L. Lynch, ${ }^{(27)}$ J. Ma, ${ }^{(3)}$ G. Mancinelli, ${ }^{(2)}$ S. Maly, ${ }^{(3)}$ G. Mantovand, ${ }^{(2)}$ T.W. Markiewicz, ${ }^{(27)}$ T. Maruyana, ${ }^{(27)}$ R. Mumetti, (20) H. Meruda, (m) E. Mazaucato, ${ }^{(20)}$ A.K. McKency, (t) B.T. Meadows, ${ }^{(3)}$ R. Mesner, ${ }^{(27)}$ P.M. Mockett, ${ }^{(31)}$ K.C. Moffeit, ${ }^{(27)}$ B. Mours, ${ }^{(27)}$ G. Müler, ${ }^{(27)}$ D. Muller, ${ }^{(m)}$ T. Nagamine, ${ }^{(m)}$ U. Naueniberg, ${ }^{(10)}$ H. Neal, ${ }^{(m)}$ M. Nusbeum, (1) Y. Obninai, ${ }^{(15)}$ L.S. Osborne, (16) R.S. Panvini, ${ }^{(30)}$ H. Park ${ }^{(20)}$ T.J. Pavel, ${ }^{(21)}$ I. Peruzzi, ${ }^{(13)(1)}$ M. Pjocoto, ${ }^{(13)}$ L. Pietrontene, ${ }^{(12)}$ E. Pieroni, ${ }^{(23)}$ K.T. Pitts, ${ }^{(20)}$ R.J. Plaoo, ${ }^{(23)}$ R. Prepost, ${ }^{(32)}$ C.Y. Preocot, ${ }^{(2)}$ G.D. Punkar, ${ }_{1}^{(27)}$ J. Quigkey, ${ }^{(15)}$ B.N. Ratclin, (2T) T.W. Reeves, ${ }^{(30)}$ P.E. Renaing, ${ }^{(2 \pi)}$ L.S. Rocthester, ${ }^{(n)}$ J.E. Rothberg, (31) P.C. Rownon, ${ }^{(11)}$ J.J. Rumell, ${ }^{(\pi)}$ O.H. Sarton, ${ }^{(27)}$ T. Schell, ${ }^{(n)}$ R.H. Schindler, ${ }_{2}^{(21)}$ U. Schneekdoth, ${ }^{(16)}$ B.A. Schumm, ${ }^{\left({ }^{39}\right)}$ A. Sciden, ${ }^{\text {(7) }}$ B. Sen, ${ }^{(35)}$ V.V. Setbo, ${ }^{(3)}$ M.H. Sheevitz, ${ }^{\text {(11) }}$ J.T. Shank, ${ }^{(3)}$ G. Shapiro, ${ }^{(5)}$ S.L. Shapiro, ${ }^{(2)}$ D.J. Sherden, ${ }^{(m)}$ C. Simopoulos, ${ }^{(m)}$ N.B. Sinev, ${ }^{(20)}$ S.R. Smith, ${ }^{(\pi)}$ J.A. Snyder, ${ }^{(20)}$ P. Stemer, ${ }^{(23)}$ H. Steiner, ${ }^{(15)}$ R. Steiner, ${ }^{(1)}$ M.C. Straues, ${ }^{(17)}$ D. Su, ${ }^{(2 \pi)}$ F. Suekane, (m) A. Sugyame, ${ }^{(1)}$ S. Suzuki, ${ }^{(19)}$ M. Swartx, ${ }^{(27)}$ A. Szumilo, ${ }^{(31)}$ T. Takahumi, ${ }^{(27)}$ F.E. Tuykor, ${ }^{(16)}$ E. Torrence, ${ }^{(16)}$ J.D. Turk, ${ }^{(23)}$ T. Usher, ${ }^{(\pi)}$ J. Va'vra, ${ }^{(27)}$ C. Vanaini, ${ }^{(21)}$ E. Velia, ${ }^{(27)}$ S.P. Veturti, ${ }^{(30)}$ R. Verdier, ${ }^{(16)}$ P.G. Vetdini, ${ }^{(23)}$ S.R. Wagnet, ${ }^{(7 \pi)}$ A.P. Waite, ${ }^{(27)}$ S.J. Watts, ${ }^{(3)}$ A.W. Weidemann, ${ }^{(20)}$ E.R. Weies, ${ }^{(31)}$ J.S. Whitaker, ${ }^{(3)}$ S.L. White, ${ }^{(20)}$

F.J. Wickens, ${ }^{(24)}$ D.A. Williams, ${ }^{(1)}$ D.C. Willians, ${ }^{(15)}$ 5.H. Willinens, ${ }^{(27)}$ S. Willocq, ${ }_{1}^{(32)}$ R.J. Wilson, ${ }_{1}^{(9)}$ W.J. Wisniewaki, ${ }^{(5)}$ M. Woods, ${ }^{(2)}$ G.R. Word, ${ }^{(25)}$ J. Wyss, ${ }^{(21)}$ R.K. Yamanoto, ${ }^{(16)}$ J.M. Yamartino, ${ }^{(16)} X$. Yang, ${ }^{(20)}$ S.J. Yellin, ${ }^{(6)}$ C.C. Young, ${ }^{(27)}$ H. Yuta, ${ }^{(20)}$ G. Zepelec, ${ }^{(32)}$ R.W. Zdarko, ${ }^{(27)}$ C. Zettlin, ${ }^{(20)}$ 2. Li. ${ }^{(16)}$ and 3. Zhou ${ }^{(20)}$

(1) Adelphi University, Garden Cily, New York I luis"

(2) INFN Sezione di Bologne, 1-10126 Bologna, thaly

(3) Boston Uniwersity, Boston, Masyachusetto 02215

(6) Brerel University. Ustridge, Middleser UB8 SPH, United Kingdom (5) Californie Institute of Technotosy, Pasadena, Californie gr1es 


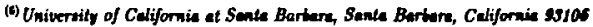

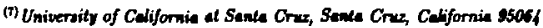

(5) University of Cincinati, Cincinnati, Ohio 45eet

(9) Colorado Stake Univervily, Fort Collin, Colonedo soses

(10) University of Colorado, Boulder, Colonedo 80509.

${ }^{(11)}$ Collsmbia Universily, New Yonk, New York 10027

(12) INFN Sezione di Fersana and Univereild di Fertere, I-11100 Fertare, Italy

(19) INFN Lab. Narionali di Frecati, L-00044 Frescati, Italy

(14) University of Mlinois, Urbera, Mllinois 61801

(18) Lawrence Benteley Labontony, Univerrity of Celifornic, Berkeley, Californie g/720

(17) Marachusetts Inotitule of Technolon, Cambrilse, Mawechusets oetsg

(17) University of Marvechwett, Amberst, Mawach wetts 01003

(10) University of Mississipgi, Univernity, Miesisoiper 98677

(19) Nagoye University, Chiture-ke, Nayoye 464 Japen

(20) Univeruity of Oregon, Evgeac, Oregon 97405

(21) INFN Sezione di Padove and Univeraild di Padou, J-95100 Padow, Ihly

(2) INFN Sexione di Perugia and Universitd di Perugia, J-06100 Perugin, Jely

(20) INFN Sexione di Pise and Universitd di Pise, I-56100 Pise, Itely

(25) Rutgers Univervily, Piscaloway, New Jersey 08855

(24) Rutherfond Appleton Labonatory, Chillon, Didcot, Oxon OX11 OQX Uniled Kingdom

(x) Sogang University, Seoul, Koree

(m) Stanford Linear Aoceleralor Center, Shanfand University, Stanford, Califormia 94909

(20) University of Tennessec, Knoxville, Tennessec 97996

(21) Tohoku University, Sendai 9s0 Jepan

(30) Vandertill Univeraily, Nahuille, Tennessee $\$ 7295$

(11) Univergily of Washington, Seatlk, Weshington 98195

(5) Universily of Wiscomsin, Medicon, Wisconsin 59706

(ख) Yale Universily, New Haven, Connecticut 05511 


\section{t Decenced}

(a) Alo at the Universild di Gemove

(1) Also at the Universild di Perygia

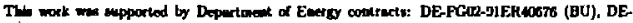

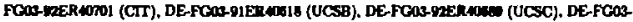

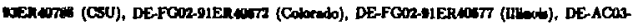

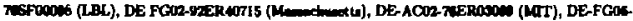

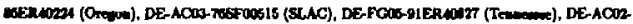

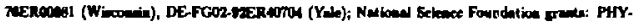

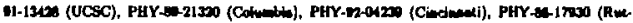

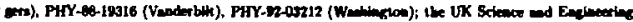

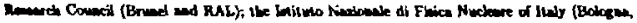

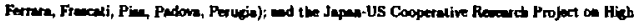
Eerory Phymias (Navogn, Tohoku). 


\section{Bibliography}

[1] A. Pais, Inwan' Bownd, Onfurd Undversity Prew, 1966.

[2] W. C. Roentgen, Sitzbger. phyeik.-ned. Ges. Würzburg, 137, Dec. 18 s.

[3] H. Bexquerel, Comples Resdues 120 (1806) 420.

[4] J. J. Thompeon, Phil. Mag. 48 (1899) 547.

[5] E. Rutherford, Phil. Mas. 21 (1911) 669.

[6] W. Pauli, letter to a physicints' gathering in Tübingen, Dec. 4, 1930.

(7) E. Fermi, Ric. Scient. 4 (1934) 491;

E. Fermi, Nuov. Cim. 11 (1934) 1;

E. Ferni, Z. Phys. 88 (1934) 161.

[8] The first experimental evideace can be found in C. S. Wu, E. Ambler, R. W. Heymerd, D. D. Hoppes, R. P. Hudeon, Phye. Rev. 105 (1957) 1413;

R. L. Garwin, L. M. Ledermas, M. Weinrich, Phys. Rev. 106 (1957) 1415;

J. I. Friedman ard V. L. Telogdi, Phys. Rev. 100 (1957) 1681;

The theoretical motivation is from

T. D. Fee, C. N. Yang, Phys. Rov 104 (1956) 254;

T. D. Lee, R. Oehme, C. N. Yans, Phyn. Rev. 105 (1957) 340.

[9] M. Gell-Marn and R. P. Fuynmun, Phya. Rev. 100 (1958) 193;

E. C. G. Sudarshen and R. E. Marahak, Phys. Rev. 100 (1958) 1860;

J. Sakurai, Nuov. Cin. 7 (1958) 649. 
(10) C. N. Yac, R. L. Mille, Phys. Rev, os (1954) 191.

[11] S. Weinberg, Phy. Rev. Lett. 19 (1967) 1264.

[12] A. Salarn, in Elementary Partide Theory Ed. N. Svatholm, p. 367, Alqvist and Wikell, Stockholm 1968 :

[13] 5. L. Glashow, Nucl. Phys. 22 (1961) 579.

[14] P. W. Hizgs, Phys. Rev, Lett. 12 (1964) 132; Phyn. Rev, 145 (1966) 1156;

for an overvies of the contributions of many of hers to the evolution of aymmetry breaking mechanisms,

M. Veltman, Proc. $6^{\text {th }}$ International Symposium on Electron and Photon Interactions at High! Enetby, Bonn, (1974) North-Holland, Amsterdam, p. 439.

[15] The ideas of apontaneous symmet; ; breuking were first introduced in Y. Nambu, Pbys. Rev. Lett, 4 (1960) 38; ;

Y. Nembu and G. Jone Lecinio, Phys. Rev. 122 (1961) 345; 124 (1961) 246;

W. Heiwenberg, Zeitscht. f. Naturf. 14 (1959) 441.

[16] G. 't Hoolt, Nucl. Phys. 35 (1971) 267.

[17] F. J. Hasert et 山., Phys. Lett. Bus (1973) 121.

[18] F. J. Hwert et al, Phys. Lett. B46 (1973) 138.

[19] J. J. Aubert et al., Phys. Rev. Lett. 33 (1974) 1404;

J. E. Augustin et al., Phy. Rev. Lett. 33 (1974) 1406.

[20) M. L. Pert et al., Phya, Rov. Lett. 35 (1975) 1489; Phys. Lett. Bes (1976) 466.

t?l] W. R. Innes et al., Phys. Rev. Lett. 30 (1977) 1240.

[22] G. Arnieon et al., Phys. Lott. B12r (1983) 103;

G. Arnieon et al, Phyn. Lett. B12S (1963) 398. 
[23] CDF Collab., F. Abe et al, Femillab-PUB-95/022-E (1905), Subarited to Phys. Rev. Lett.;

D0 Collab., S. Abrehi et a., Fermilab-PUB-95/020-E (1985), Submittei to Phys. Rev. Lett..

[24] C. Bouchiat, J. Iliopoulos, and P. Meyor, Phys. Lett. Bss (1972) 519;

D. J. Grow and R. Jeckiw, Phys. Rev. Do (19T2) 477;

H. Georgi and S. L. Gimbow, Phys. Rev, De (1972) 429.

[25] F. Halzen, A. D. Martin, Querte 8 Leptone, John Wiley 4 Sone, Inc, 1994.

[26] C. Quigs, Gange Theories of the Strong, Weak, and Electromegnetic Inter. ection, Frontiers in Physics, The Benjamin-Cumming Publinhing Co., Inc., 1933.

[27] J. Goldistone, Nuowo Cirn. 10 (1961) 246;

J. Gokdstone, A. Salum, and S. Weinberg, Phys. Rev. 127 (1962) 965.

[28] E. R. Cohen and B. N. Taylor, Rev. Mod. Phyn. 50 (1987) 1121.

[29] K. Hikase et al., Phys. Rev. D45, Part II (1992)

[30 Review of Particle Properties, Phys. Rev. D\$0 (1994) 1356.

[31] J. H. Kühл, P. Zerwe, is $Z^{0}$ Phyoics at LEP I, G. Alterelli, et d. (edw), CERN Yellow heport 89-08, Vol 1. (1999).

[32] M. E. Peskin, Theory of Precirion Electrowed Measurements, SLAC-PUB5210 (1990).

[33] G. Bonneau and F. Martin, Nucl. Phys. Bat (1971) 381.

[34] E. A. Kureev and V. S. Fudin, Sov. J. Nucl. Phys. 41 (1985) 466.

[35] D. C. Kennedy, J. M. In, B. W. Lynn, R. G. Sturer, Nucl. Phys. Bs21 (1000) 83. 
(36] W. F. L. Hollik, Fort. Phys, 38 (1990) 165;

A conclse summary of this work can be found in W. F. L. Hollik, in Electroweal Physics Beyond the Standand Model, Valencia, Spain, J. W. F. Valle and J. Velasco, eds., p. 57, World Scientific, Singapore, 1991.

[37) A. A. Akbundow, D. Yu. Bardin, and T. Riemann, Nucl. Phyf. D276 (1996) 1, and references therein.

[38] D. Kennedy and B. W. Lynn, Nucl. Phys. B322 (1989) 1.

[39] F. J. Dyson, Phys. Rev. 75, (1949) 1736.

[40] N. Cabbibo and R. Gatto, Physs. Rev. 124 (1961) 1577.

[41] M. Swartz, Reevaluation of the Hadronic Contribution to $\alpha\left(M_{Z}^{2}\right)$, SLAC-PUB6710, submitted to Phys. Rev. D.

[42] A. Sirlin, Nucl. Phys. B71 (1974) 29; Nucl. Phys. B100 (1975) 291.

[43] J. J. van der F" 'nd F. Hoogeveen, Nucl. Physs. 9283 (1987) 477.

[44] W. Beenakker is a IV. Hollik, in 2 Physics at LEP 1, Getev, 1989, G: Altarelli, R. Klelss, and C. Verzegnassi, eds., CERN Report No. CERN-89-08, vol. 3.

[45) B. W. Lynn, M. E. Peakin, and R. G. Stuart, in Physics at LEP, J. Ellis and R. Peccei, eds. CERN Report CERN 86-02 (1986); SLAC-PUB-3725 (1985).

[46] B. W. Lynn and C. Verzegnassi, Phys. Rev, D35 (1987) 3 226.

[47) SLD Collab., K. Abe et aL, Phyb. Rev, Lott. 73 (1994) 25.

[48] Sce, e.g.n W. Bartel et al., Phys. Lett. BeO (1981) 281;

M. Levi et al, Phys. Rev. Lett. 51 (1985) 1941.

[49] A. Blondel, B. W. Lynn, F. M. Renard, and C. Verzegnassi, Nucl. Phys. B304 (1988) 438. 
[50] F. M. Renard, A. Blondel, and C. Verzognemi, $Q E D$ and $Q C D$ Effects in POlarized Forwand-Backwand Asymmetries, in Palaritation at LEP, G. Alexandor et al., cols., CERN Report No. CERN-99-06, ml. 1.

[51] 2FITTER: D. Bardin et ah, CERN-TH. 6443/92;1992).

[52] J. B. Stav, and H. A. Oloen, private communication.

[53] A. Djoutud, J.H. Kihn, P.M. Zerwes, Z. Phys. C 40 (1990) 411, and references tharein.

[54] G. Altarelli and B. Lampe, Nuclear Physics B391 (1993) 3.

[55] G. Burdman, E. Golowich, J. L. Itewetl, S. Pakvass, Using Charm to Prabe Net Physics, Plyss. Rep. (In preparation).

;56| For general reviews of supersymmetric phenomene, sce

J. F. Gunion et al.The Higgs Henter's Guide, Frontiert in Physics, AddioonW'sley Publishing Company, Inc., 1990, H. E. Haber and G. L. Kane, Phys. Rep. 117 (1985) 117.

[57] M. Boulware and D. Finnel, Phys. Rev. D44 (1991) 48.

[58] See, e.g., L. J. Hall and S. Weinberg, Phys. Rev. D48 (1993) 979; A. Antaramian, L. J. Hell, and A. Rasin, Phys. Rev. Lett. 69 (1992) 1871; W. S. Hou, R. G. Stuart, Nucl. Phys. B320 (1989) 277; T. P. Chen and M. Sher, Phys. Rev. D35 (1987) 3484.

[59] For a general review of string-theory-inspired $E(6)$ models, see

J. L. Hewott and T. G. Rizzo, Phys. Rep. 183 (1989) 193.

[60] E. Ma, Phys. Lett. B944 (1995) 164;

E. Ma and D. Ng, Phys. Rev. D39 (1989) 1986.

[61] M. E. Nelson et al, Phys. Rev. Lott. 50 (1983) 1542.

[62] E. Farhi, Phys. Rev, Lett. 301587 (1977). 
[69) ALEPH Collab., D. Buskulic et alZ. Phys. C62 (1994) 179;

DELPHI Collab., P. Abreu et \&.Phys. Lett. Ba70 (1992) 536;

L3 Collab., O. Adrianl et L Phys. Lett. Bazz (1992) 494;

OPAL Collab., R. Akets et al, Z. Phys. Cos (1992) 19;

[64) G. Feldman et $\alpha$, Phys. Rev. Lett. 83 (1977) 1313.

[65] See, e.9., G. Goldhaber, Proceedings of the XVIIth Rewcontres de Moriond, La Plegne, 1983.

[66] Particle Datn Group, Review of Particle Properties, Phys. Rev, D50 (1994) 1173.

[67] ALEPH Collab., D. Buskulic et aL, Z. Phys, Co2 (1994) 1.

[68] DELPHI Collab., P. Abret et al, CERN-PPE-94-193 (1994).

[69] OPAL Collab., R. Akers et af, 2. Phys, Ceo (1993) 601.

(70) ALEPH Collsh., D. Buskulic ef c .hyr. Lett. BS13 (1993) 535.

[71] TASSO Collab., R. Brandebik et al, Phys. Lett. B100, (1981) 357.

[72] ALEPII Collab., D. Buskulic et aL, Phys. Lett. B335 (1994) 99; DELPHI Collab., P. Abreu et al, CERN-PPE-94-131.

[73) SLD Collab., $K$. Abe et al, Measurement of $A_{4}$ and $A_{c}$ from the Left-Right Forward-Backuard Asymmetry of $b$ Quark Production in $Z^{0}$ Deoays Using a Momentwm-Weighted Track Charge Technique, SLAC-PUB-6644, to appent in Phys. Rev. Lete.

[74] SLD Collab., $K$. Abe et al., Measurement of $A_{4}$ and $A_{\varepsilon}$ from the Left. Right Forward-Bachoord Asymmetry of Leptons in Hadronic Euents at the $Z^{0}$ Resonance, SLAC-PUB-6607, to appear in Phys. Rev. Lett.

[75\} J. Chadwick, Nuture 12: (1932) 312.

[76] H. Yukawa, Proc. Phys. Math. Soc. Japan 17 (1935) 48. 
[77) M. Gell-Mann, Phyn. Lett. (1964) 214;

G. Zweig, CERN s162/TH, Januny, 1964;

G. Zweic, CERN 8419/TH, Fobruer, 1964.

[78] M. Y. Hen and Y. Nambu, Phys. Rev. 130B (1965) 1006.

(79) E. D. Bloom et al., Phys. Rev. Lett. 23 (1960) 930;

M. Bredienbach et el, Phys. Rev. Lett. 23 (1960) 935.

[80] D. J. Groes, F. Wikenek, Phyz. Rev. Lett. 30 (1973) 1343;

H. D. Politzer, Phys. Rev. Lett. 30 (1973) 1346.

[81] TASSO Collab., R. Brandelik et ah, Phya. Lett. Bas (1979) 243;

MARK J Collab., D. P. Barber et d, Phy. Rev. Lett. 43 (1979) 830;

PLUTO Collab., Ch. Berger of al, Phy. Lett. Bes (1979) 418;

JADE Collab., W. Bartel et al, Phys. Lett. Bo1 (1900) 142.

[82] R. D. Field, Applications of Perturiative QCD, Frontiers in Phyice, AddisenWealey Publishing Compeny, Inc., 1989.

[83] J. Ellis, M. K. Gaillerd, G. G. Roes, Nucl. Phyn. B111 (1976) 253. A detailed derivation can also be found in Ref [R2, p. 25].

[8] G. Sterman, S. Weinberg, Phys. Rev. Lett. 3e (1977) 1436.

[85] Z. Kunozt, Phys. Lett. Beo (1981) 479; B107 (1981) 123.

[86] T. Kinoshite, J. Math. Plys,, 3 (1962) 650.

T. D. Lee, M. Nauenberg, Phys. Rev, B133 (1964) 1547.

[87] A. Ali et al, Nucl. Phys. B167 (1900) 454;

K. J. F. Geemers, J. A. M. Vermueren, Z. Phys. C7 (1960) 81;

R. K. Ellis, D. A. Roos, A. E. Terrano, Nicel. Phys. B17s (1981) 421;

D. Duncksert, Phys. Lett. B114 (1982) 203.

[B8] K. Hagiwara, Nucl. Phys. B220 (1969) 550;

F. A. Berends, Nucl. Phys. B231 (1900) 39; 
[80] G. Kramer, Theory of Jets in Electron-Positron Annihitation, Springer-Ver]ag, Heidelberg, 1924.

[90] G. 't Hooft and M. Veltmen, Nucl. Phys. B44 (1972) 189.

[91] W. J. Nercieno, Phys. Rev. D12 (1975) 3861.

[92] G. 't Hooft, Nucl. Phys. Baa (1973) 455.

[93] W. A. Bardeen et al, Phys. Rev. D18 (1978) 3998.

[94] G. Altere]li, G. Parisi, Nucl. Phys. B12t (1977) 298.

[95] The leading lozaritbmic terms in the deep-inelentic scattering structure functions was first calculated by:

H. Georgi, H. D. Politzer, Phys. Rev. D9 (1974) 416;

D. J. Gross, F. Wilcakt, Phys. Rev. D9 (1974) 990.

[96] V. N. Gribov, L. N. Lipatow, Sov. Nucl. Phys. 15 (1972) 438, 675;

Yu. L. Doloshitzer, D. 1. D'yenkonov, S. I. Troynen, Phys. Rep. 58 (1980) 269, and references thetein.

[97] D. Amnti et al, Nucl. Phys. B17s (1980) 429.

[98] Particle Data Group, Review of Particle Properties, Phys. Rev. D50 (199) 1297.

[99] J. D. Bjorken, Phys. Rev. 179 (1969) 1547.

[100] S. Coleman, D. J. Groes, Phys. Rev. Lett. 31 (1973) 851;

A. Zee, Phys. Rev. D7 (1973) 3630.

[10i] S. Weinberg, Phys. Rev. Lett. 31 (1973) 494;

D. J. Gros6 and F. Whikack, Phys. Rev. D8 (1973) 3633;

H. Fritzsch, M. Gell-Mann, and H. Leutwyler, Phys. Lett. B47 (1973) 365;

H. Georgi and S. L. Glenhow, Phys. Rev, Lett, 32 (1973) 438.

[102] D. J. Groen, A. Neveu, Phys, Rev. D10 (1974) 3235. 
[103] G. Hanson et al., Phys. Rev. Lett. 35 (1975) 1609.

[104] For a review of this subject, wee T. Sjöstrand, Int. J. Mod. Phys. A3 (190) 751.

[105] M. Begtwoon, T. Sjöstrand, Nucl. Phys. Bases (1987) 810, and reference therein.

[106] A. H. Mueller, Phys. Lett. B104 (1931) 161;

B. I. Ermoleev, V. S. Fudin, JETP Lett, 33 (1981) 209 ;

L. A. Gribow, E. M. Levin, M. G. Ryskin, Phys. Rep, 100 (19e3) 1;

A. Benetto, M. Ciafaloni, G. Merchesini, Phys. Rep. 100 (1983) 201.

[107] R. D. Field, R. P. Feynman, Nucl. Phys. B13s (1978) 1.

[108] P. Hoyer etal, Nucl. Phys. B161 (1979) 349.

[109] A. Ali etal, Nucl. Phys. bf B168 (1900) 499;

A. Ali et al, Phys. Lett. Bos (1900) 155.

[110] F. E. Prige, S. D. Protopopesed, in Phyoics of the Srgarcondiction Supercollider 1986, eda. R. Donaldson, J. Marx (1987) p. 320.

[111] X. Artru, G. Mennesier, Nunl. Phys B70 (1974) 93.

[112] X. Artru, Phys. Rep. 03 (1983) 147.

[113] T. Sjöstrand, Comput. Phys. Comm. 30 (1966) 347;

T. Sjattrand, M. Bengeston, Comput. Phys. Comm. 43 (1987) 367.

[114] B. Anderason, G. Gustafion, C. Peterson, 2. Phys. C1 (1979) 105.

[115] See B. Andenseon, G. Gustafion, C. Peterson, Z. Pbys. Co (1960) 235, and references therein.

[116] A. Petersen, in Elementary Contituents and Hadronic Structure, od. J. Tran Thanb Van (Editions Frontitra, Dreux, 1900) p. 505. 
[117] Sec, for example, OPAL Collab.: M. Z. Akrawy et $\alpha$, Phys. Lett. B2s1 (1991) 334.

[118] G. Marchesini, B. R. Webber, Nucl. Phys, B238 (1984) 1.

[119] B. R. Webber, Nucl. Phys. B23s (1984) 492.

[120] G. Marcheani et alCambridge Report Gavendish-HEP-90/26 (1990); DESY Report DESY-01-048.

[121] B. Andersoon, G. Gustafion, C. Peterson, Z. Pbys. C20 (1983) 317.

[122] C. Peterson, et al., Phys. Rev D27 (1993) 105.

[123] The LEP Electroneak Heavy Flevors Working Group, D. Abbaneo et al., Presentation of LEP Elextruxcak Heniy Flavor Results for Summer 199/ Conferences, LEP-HF/94-02 (1994), and reference therein.

[124] A concise summary of the experiments program of QCD studies cen be found in: S. Bethke, Experimental Overview of Jet Physics and Test of QCD, CERNPPE/91-36 (1991).

[125] PLUTO Collab., Ch. Bergir et \&, Phys. Lett. Bor (1980) 459.

[126] JADE Collab., W. Bartel et af, Zeit. Phys. C33 (1986) 23.

[127] G. Kramer and B. Lampe, Z. Phys. Cs9 (1988) 101;

N. Magnussen, DESY Report F22-89-01 (1989);

5. Bethke, Z. Phys. C43 (1989) 331;

For the method of resummation, see:

5. Cateni, G. Turnock, B. R. Webber, and L. Trentadue, Phys. Lett. B2as (1991) 491;

S. Caturi, G. Turnock, B. R. Webber, Phya. Lett.B272 (1991) 368;

S. Catani et al., Phys. Lett.B2es (1991) 432;

S. Catani, G. Turnock, B. R. Webber, CERN-TH-6570/92 (1992);

G. Turnock, Covendinh-HEP-92/3 (1992); 
S. Catani, L. Trentedue, G. Turpock, and B. R. Wobber, Nucl. Plyn. Bat (1993) 3.

[128] Z. Kunst, P. Neon, G. Murchesini, and B. R. Webber, in Proceding of the Workshop on $\mathrm{Z}$ Physics at LEP I, ed. G. Altarelli, R. Kloim, and C. Veragegnen, CERN-89-08, (1999) ver. 1, p. 414.

[129] G. Kramer and B. Lampe, 2. Phys. C34 (1987) 497 [Erratum: C42 (1900) 504]; Fortsechr. Phys. 37 (1989) $16 \mathrm{t}$.

[130] T. Appelquist and H. Georgi, Phys. Rev. Do (1973) 4000;

A. Zee, Phys. Rev, Do (1973) 4038;

M. Dine and J. Supirstein, Phya. Rev. Lett. 43 (1979) 668;

W. Celmaster and R. J. Gonenlve, UCSD preprints IOP10-206,207 (1979);

K. G. Ghetyrkin, A. L. Kateev, F. V. Thachov, Phyw. Lett. Bos (1979) 277.

[131] SLD Collab., K. Abe et al, Measurement of o,(M) from Hedronic Event $O_{b}$. servilies at the $Z^{\circ}$ Resonance, SLAC-PUB-6611 (1994); to spear in Phys. Rev. D.

[132] J. J. Aubert et al., Phys. Rev. Lett. 33 (1974) 1404;

J. E. Augustin et al., Phys. Rev. Latt. 33 (1974) 1406.

[133] S. L. Adlex, in Lectures on Elementary Particle Phyoics in Guentum Fied Theary, Brundeis 1970, p. L, M.L.T. Prem, Cembeidge, M=. 1970.

[134] See, for exnmple, J. Kuti and V. Weiskopd, Phys. Rev. DA (1971) 3418.

[135] TASSO Collab., R. Brandelik et ah, Phys. Lett. Bet (1960) 453;

PLUTO Collab., Ch. Berger et ah, Phys. Lett. Bot (1960) 459;

CELlo Collab., H. J. Behrend et a., Phys. Lett. B110 (1982) 329.

[136] JADE Collab., S. Betlke et L, Phys. Lett. B213 (1988) 235.

[137] AMY Colleb., J. Park et LL, Pbys. Rev. Lett. 6 (1939) 1713. 
[138| J. Ward, Phys. Rev. 73 (1950) 182.

Y. Tublanki, Nuovo. Cin. 6 (1957) 371.

[139] The earlieat calculations of the Ward-Taknhahi identities for Yang-Mills theories eppent in:

J. C. Taylor, Nuc. Phys. B33 (1971) 436;

A. Slavnov, Kiev Report No. ITP-71-83E; Theor. Mlath. Phys. 10 (1972) 99;

B. W. Lee, J. Zinn-Justin, Phys. Rev, Ds (1972) 3121.

[140] M. E. Peskin, D. V. Schroeder, An Intraduction to Quantum Field Theory, Addison-Wesley (1995?).

[141] T. G. Rizzo, Phys. Rev. Dso (1994) 4478;

Yu. L. Dokshitzer, V. A. Khoze, and W. J. Stirling, University of Durharm Report No. DTP/94/14 (1994).

[142] TASSO Collab., W. Braunschweig ct al., Z. Phys. C44 (1989) 365.

[143] TASSO Collab, W. Braunsclweig et al., Z. Phys. C42 (1989) 17.

[144] L3 Collab., B. Adeva et al., Plys. Lett. B271 461 (1991);

DELPHI Collab: P. Abreu et al., Phys. Lett. B307 (1993) 221.

[145] OPAL Collab., R. Akers et al, Z. Plyys. Co0 (1993) 397.

[146] OPAL Collnb, R. Akers et of, CERN-PPE/94-123 (1994).

[147] J. E. Augustin et al., Limitations on Performance of $\mathrm{c}^{+} e^{-}$storage rings and Linear Colliding Beam Systems at High-Eneryy, in Batavia 1978 Proccedings, "Possibilitios and Limitations of Accelerators and Detectors", Batavia, 1979 p. 87; B. Richter, Conceptual Design of a Linear Colliding Beam System to Reach $100 \mathrm{GeV}$ in the Center-of-Mass, SLAC-AATF/79/03 (1979).

[148] A schematic vicw of the recirculation system proposed for the TESLA collider can be found in R. Brinkman, TESLA Rexirulation Scheme, Talk presented at the Fifth International Workshop on Next Generation lincar Colliders (LC93), SLAC-Report-436 (1993) 432. 
[149] D. T. Pierce, F. Meier, and P. Zürcher, Phys. Lett. A51 (1975) 465.

[150] D. Schulzz et al, The Polarized Elactron Source of the Slanforl Limeer Acollerator Center, SLAC-PUB-6606 (1994).

[151] SLD Collab., K. Abe et al., Pby:. Rev. Lett. 70 (1993) 2515.

[152] T. Maruyaran et al, Pbys. Rev. B4S (1992) 4246.

[153] T. Limberg, P. Enoma, R. Rosmanith, The North Arc of the SLC as E Spin Rotator, in Procedings of the 1993 Particle Accelerator Conference (PAC 93), Weshington, DC, 1993; SLAC-PUB-6210.

[154] J. Kent et al, Precision Measurement of the SLC Beam Enery, SLAC-PUB4922, (1989).

[155] F. Taylor, A First Onder Calculation of Compton Scattering Asymmetry, SLD Physics Note \# 26 (1993).

[156] M. Fero et al, SLAC-PUB-6423, (1995).

[157] W. R. Nelson et al, The EGS/ Code System, SLAC-Report-265 (1985).

[158] D. L. Burke et al., First Results from the Find Focus Test Bom, to appear in the procedings of the 1994 Europen Particle Acoeterator Conference (EPAC 94), London, 1994; SLAC-PUB-6609 (1994).

[159] SLD Design Report, SLAC-Report-273 (1984).

[160] See M. G. Straus, Pefformence of the SLD CCD Pizel Vertex Detector and Design of an Upyrade, SLAC-PUB-6696 (1994), to sppear in the Proceeding of the 27th ICHEP, Glasgow, 1994, and references therein.

[i61] S. G. Berridge et al., JEEE Trans. on Nucl. Sci. NS-39 (1992) 1242.

[162] K. T. Pitts, Elextrowent Cowpling Measurements from Polarized Bhathe Soattering at the $Z^{0}$ Resonance, Ph.D. Thesis, University of Oregon (1994), SLACFeport-446. 
[163] M. D. Hildreth et al, Performance of the SLD Centril Drift Chamber, SLACPUB-6659 (1994), submitted to 1:EE Trans. on Nucl. Sci.

[164] A description of the drift chamber readout electronics ean be found in G. M. Holler et al, IEEE Trans. on Nucl. Sci. NS-38 (1991) 363, and references therein.

[165] J. P. Venuti and G. B. Chadwick, Radintion Aging Studies of $\mathrm{CO}_{2}$ Hydrocarbon Mirtures for the SLD Drift Chamber, IEEE Trang. in Nud. Sei. NS-30 (1989) 595.

[166] D. C. Willians, The Left-Right Forvard-Backwand Asymmetry for B Quarks at the SLD, Ph.D. Thesis, MIT (1994), SLAC-Report-445, Appendix C.

[167] See M. Dracos and D. Loukas, Nucl. Inst. Meth. A302 (1991) 241, and references therein.

[168] M. CavaLi-Sforza et al., IEEE Trans. on Nucl. Sci. 37, (1990) 1132;

K. Abe et al. SLAC-PUB-6399 (1993).

[169] D. Axen et al. Nucl. Inst. Meth. \& $\quad$ ' (1993) 472.

[170] S. Gonzalez Martirena, A Calorimetric Measurement of the Strong Coupling Constont in Electron-Positron Amnihilation at a Center-of-Mass Energy of 91.6 GeV, Ph.D. thesis, M.I.T.. (1994); SLAC-Report-439.

[171] Harvey Lỵch, private communication.

[172] A. C. Benvenuti et al., Nucl. Instr. Meth. A276 (1989) 94. An even more detailed description of the WIC and its use as a muon tracking bystera is given in Reference [166], Chapter 5.

[173] R. Brun et al. GEANTS, CERN-DD/EE/84-1 (1987).

[174] For a written description of the pattem recoguition algorithm, see Ref. [166], Appendix B. 
[175] P. Billoir, Nucl. Inst. Meth. 225 (1984) 352.

[176] The $V^{0}$ selection analysis was performed by Ken Baird. See K. G. Beird, $A$ Study of $K_{a} \Lambda$, and $\bar{\Lambda}$ Production in Hadronic $Z^{0}$ Docays, SLAC-PUB-6001 (1994).

[177] SLD Collab., K. Abe ef al, Measurement of the $\tau$ Lifetime at SLD, SLACPUB-95-6767 (1995).

[178] J. M. Yamartino, A Mearurement of the $e^{t} e^{-}$Decay Width of the $Z^{0}$, Ph.D. Thesis, MIT, February 1994; SLAC-Report-426, Ghapter 4; J. M. Yamertino, Hadronic Event Selection Uring the LAC, SLD Physics Note 14, 1993.

[179] Some reference by tracy? This way of searching rapidly for charged tracks is sirailar to tbat implemented in hardware by the Mark Il collaboration, in their so-called "curvature modules". See T. Himel, Decnys of the $\psi(3684)$ to Other Charmonium States, Ph.D. Thesis, SLAC-Report-223 (1979).

[180] P. N. Burrows, H. Park, K. T. Pitts, J. M. Yamartino, Estimate of Combined Thiggering and Seloction Efficiency for Hadronic Bvents, SLD Note 229, (1993).

[181] Details of the $\boldsymbol{K}^{0}, \boldsymbol{\Lambda}$ and $\gamma$-conversion finder used in this analysis can be fourd in D. S. B-fractim of the $Z^{0}$, Proceedings of the SLD Collaboration Mecting, Vol 2, Saratoga. WA, Feb. 1994, and will appear in H. Neal's Stanford Ph.D. thesis.

[182] SLD Collab., K. Abe et al., Measurements of $R_{6}$ with Jmpact Parameters and Displaced Vertices, SLAC-PUB-6569, submitted to Phys. Rev. D.

[183] ALEPH Collab., D. Tecamp et al, Phys. Lett. B273 (1991) p.181.

[184] OPAL Collab., P. D. Acton et al, Z. Phys. C53 (1992) p. 539.

[185] K. Hayes, B Tagging at the SLC, Mark 1I/SLC Note 73, May 8, 1984, unpublished; K. Hayes, $b$ and c Quark Exclusive Decuys with the Vertex Deteclor, in Procedings of the Third Mark II Workshop on SLG Physies, SLAC-Report-315, July 1987. 
[186] See, for example, Mark II Collab., R. G. Jacobeen et al., Phys, Rev. Lott, 67 (1991) p. 3347, and R. G. Jacobsen, A Measurement of the Branching fraction of the $Z$ Boson to Bollom Quarks Vsing Precision Thading, Ph.D. Thesis, Stanford University, July 1991, SLAC-Report-381.

[187] S. Bethke el al, Nuel Phys. B370 (1992) 310.

[188] Mark II Collab., S. Komamige et al., Phys. Rey. Lett. 64 (1990) 987.

[169] B. L. Ioffe, Plyys. Leî. B78 277 (197B).

[190] E. Laemann, P. M. Zerwas, Phys. Lett. B89 225 (1980).

[191] A. Balles rero, E. Maina, S. Moretti, Phys. Lett. B294 425 (1992).

[192] For a readable account of this calculation, see B. de Wit and J. Smith, Field Theory in Particle Physics, North Holland, Amsterdam, 1^86, Section 8.6.

[193] A. Ballestrero, E. Maina, S. Moretti, Nucl. Phys. B415 (1994) 265.???

[194] The Particle Data Group, Phys. Rev. D50 (1994).

[195] ARGUS Collab., H. Albrecht et al., Z. Phys. C54 (1992) 13.

[196] ALEPH Collab., D. Buskulic et of, CERN-PP/94-107 (1994);

L3 Collab., B. Adeva et al., Phys. Lett. B261 (1991) 177;

OPAL Collab., R. Akers et aL, Z. Phys. C60 (1993) 199;

OPAL Collab., R. Akers et al, CERN-PPE/93-174 (1993).

\{197\} This result is derived from a consideration of the JETSET parameters.

[198] ALEPH Collab., D. Busk he at, CERN-PP/93-208 (1993);

DELPHI Collab., P. Abreu et al., Z. Phys. C59 (1993) 533;

OPAL Collab., R. Akers et al, Z. Phys. C61 (1993) 601.

[199] W. Venus, to Weak Interaction Physies at High Energies", Proccedings of the 16 ${ }^{\text {h }}$ Symposium on Lepton and Photon Interactions, Cornel] University (1993), p.274; Eds. P. Drell and D. Rubin (AIP 1993). 
[200] Markll Collst., D. Coffman et al., Phys. Lett. B203 (1991) 135.

(201] HRS Collat.. S. Abachi et al., Phys. Lett. 8205 (1988) 411.

[202] See, c.g.. A G. Frodesen, O. Skjeggestad: Probabilty and Statistice in Particle Physics, L'niversitetsforlaget Bergen-Onlo-Tromso (1979).

[203] R. Akors, et al., Z. Phys. C 60 (1933) 601.

[204] D. Scheile, to appenr in the proceedings of the XXV1/ th ICHEP, Glasgow 1994.

[205] H. G. Moser, Proc. $5^{\text {th }}$ Iut. Symp. Heary. Flav. Physics, Montreal (-993) p. 585.

[206] OPAL Collab: G. Alexandor. Bt M1. Phys. Lett. B262 (1991) 341.

[207] F.M. Renard, A. Blondel, and C. Verzegnnssi, in Polarization at LEP, G. Alexander (ed.), Vol. 1, pp 197-203.

[208] K. Moentg, 'Deterimination of $\sin ^{2} \theta_{w}$ from Forward-Backmard Asymmotries with Quarks and Leptons at LEP, 27th Interantion Conference on High Energy Physics (ICHEP), Glasgow 1994.

[209] OPAL Collab., R Akers et al, CEßN-PPE/94-217 (1994).

[210] C. Adolphsen et al, "Flat Beams in the SLC", SLAC-PUB-6118, Presented at the Particle Accelentor Couference (PAC 93), Washington, D.C., Muy, 1993.

[211] Jacobecn et al, "Detector Background Conditions at Linear Colliders", SLACPUB-5205, (1990).

[212] G. Feldman, Muon Backgrounds ot the SLC:, Mark II Collaboration loternal Memo, 3/23/88, (Unpublished).

[213] Saul Gonzalez, "Report on Muon Background Monte Carlo Study", SLD Internal Note 214, (1902) (Unpublished').

[214] W.R. Nelson et al., "The EGS\$ Code Sysiem". SLAC Report 265, (1965). 


\section{BLBLIOGRAPHY}

[215] The SLD Colluboration, Proposel to Upgrade the SLD Vetex Detector ${ }^{n}$, Octoun 1993. 